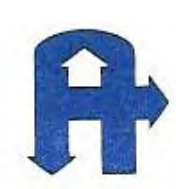

Advanced Consulting Engineering Services, Inc.

\title{
Task 9: Update and Improve Subsection NH - Simplified Elastic and Inelastic Design Analysis Methods
}

\section{Subtask 9.1 - Outline of an "Ideal” High Temperature Code}

\author{
Gen IV / NGGNP Materials Project \\ Submitted to \\ Mr. Jim Ramirez \\ Vice President, Business Development \\ ASME Standards Technology, LLC \\ Three Park Avenue \\ New York, NY 10016 USA
}

\author{
By \\ Advanced Consulting Engineering Services, Inc. \\ Peoria, IL \\ On behalf of \\ Jeries J. Abou-Hanna \\ Douglas L. Marriott \\ Timothy E. McGreevy
}

July 21, 2008 


\section{TABLE OF CONTENTS}

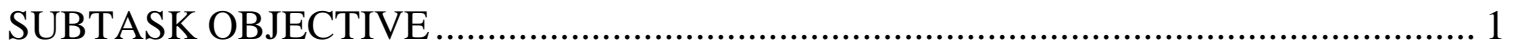

ELEMENTS OF THE “IDEAL” HIGH TEMPERATURE CODE ...................................... 2

1. DEFINITION OF "HIGH TEMPERATURE”........................................................ 2

2. DESIGN LOADS \& FAILURE MECHANISMS TO CONSIDER ……………......... 4

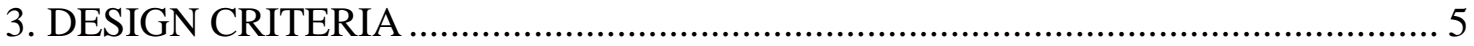

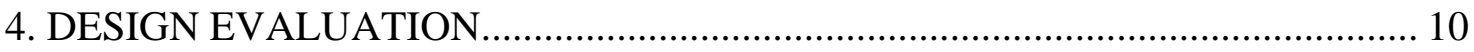

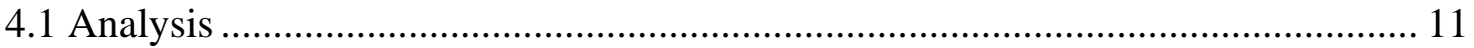

4.1.1 Level 1 Fundamental Design ........................................................................ 12

4.1.2 Level 2 Detailed Design............................................................................. 13

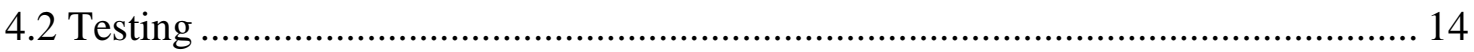

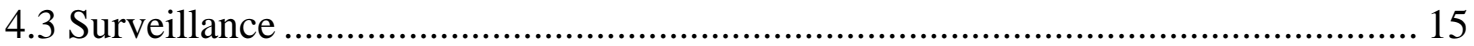

5. MATERIAL PROPERTIES REQUIRED TO PERFORM A DESIGN

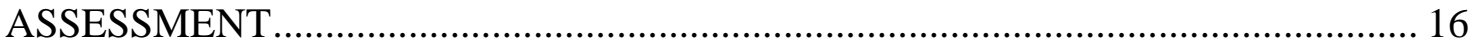

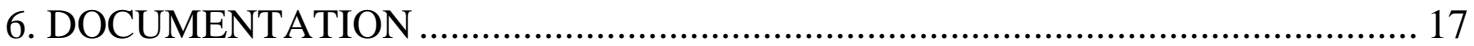

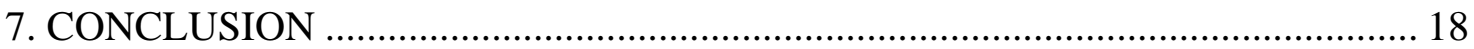




\section{SUBTASK OBJECTIVE}

The objective of this subtask is to develop a template for the "Ideal" high temperature design Code, in which individual topics can be identified and worked on separately in order to provide the detail necessary to comprise a comprehensive Code.

Like all ideals, this one may not be attainable as a practical matter. The purpose is to set a goal for what is believed the "Ideal" design Code should address, recognizing that some elements are not mutually exclusive and that the same objectives can be achieved in different way. Most, if not all existing Codes may therefore be found to be lacking in some respects, but this does not mean necessarily that they are not comprehensive.

While this subtask does attempt to list the elements which individually or in combination are considered essential in such a Code, the authors do not presume to recommend how these elements should be implemented or even, that they should all be implemented at all.

The scope of this subtask is limited to compiling the list of elements thought to be necessary or at minimum, useful in such an 'Ideal' Code; suggestions are provided as to their relationship to one another. Except for brief descriptions, where these are needed for clarification, neither this repot, nor Task 9 as a whole, attempts to address details of the contents of all these elements. Some, namely primary load limits (elastic, limit load, reference stress), and ratcheting (elastic, e-p, reference stress) are dealt with specifically in other subtasks of Task 9. All others are merely listed; the expectation is that they will either be the focus of attention of other active DOE-ASME GenIV Materials Tasks, e.g. creep-fatigue, or to be considered in future DOE-ASME GenIV Materials Tasks.

Since the focus of this Task is specifically approximate methods, the authors have deemed it necessary to include some discussion on what is meant by "approximate". However, the topic will be addressed in one or more later subtasks. 


\section{ELEMENTS OF THE “IDEAL” HIGH TEMPERATURE CODE}

The report for subtask 9.1 is written with a section addressing each of the following topics:

1. A definition of "high temperature"

2. A comprehensive list of the failure mechanisms and other conditions to be considered in design for high temperature operation

3. A list of design criteria to be complied with in assuring the acceptability of a component designed for operation at high temperature

4. Evaluations, including but not necessarily restricted to analyses, testing and surveillance, as required to provide assurance that a design complies with Code criteria.

5. Material properties required to perform a design assessment

6. Documentation demonstrating Code compliance, including both document management by the Code body itself, such as updating and revisions, as well as user records demonstrating compliance.

\section{DEFINITION OF “HIGH TEMPERATURE”}

"High temperature" is taken to refer here to the operating range of temperature within which time dependent, thermally activated deformation and damage processes, even under nominally steady loads below yield, become a significant factor in the behavior of load bearing components.

This definition is commonly taken to mean the appearance of creep as a significant mechanism, but others, such as thermal ageing and oxidation/corrosion are also important.

In practice time dependency is a smooth function of temperature. Consequently there is no clear context free boundary separating time-independent from time-dependent 
behavior. For specific forms of service conditions and material response, it is possible to define a temperature limit below which "high temperature" may be considered negligible in that the time dependent phenomena associated with high temperature behavior do not have a significant effect on design decisions. For the purpose of defining the applicability of a high temperature design Code, the threshold temperature marking the point above which time dependency first reaches significance is one valid criterion defining "high temperature" design.

The threshold temperature is not unique. It is strictly a function of the mode of failure being considered, as well as the design lifetime. For instance, the threshold temperature for constant loading conditions, where stresses are expected to relax to a relatively low steady state, will be higher than one based on cyclic conditions which cause stresses to be repeatedly reset to the yield stress by cyclic plastic deformation. The specified design lifetime will also influence the threshold temperature. A higher value might be tolerated for short service lives compared with extended lifetimes.

These are only the factors determining the importance of time dependent behavior in general. How these factors are implemented in a design Code is a decision to be made by the Code drafting body. All this report aims to do is to list current practices or Code definitions.

At one extreme, it is conceivable that the criterion for moving to a high temperature Code could depend on whichever application specific failure mechanism yielded the lowest threshold temperature. For example, if the only condition seen by the component is steady loading, a higher limit might be permitted than if cyclic conditions were expected. A simpler but possibly more conservative option is to select a single generic criterion based on the mechanism found to be the most conservative of all load cases covered by the Code, regardless of application. Finally, a Code might adopt a hybrid option, allowing less conservatism, at the cost of more effort to justify the use of a higher limit. A service life with limited period at elevated temperature, or a component with a short specified lifetime, might qualify for such treatment. 


\section{DESIGN LOADS \& FAILURE MECHANISMS TO CONSIDER}

Design encompasses many aspects of system development and operation. This document is restricted in its scope to criteria governing structural integrity or avoidance of mechanical failure due to structural collapse or material damage.

"Load" is assumed here to include stress inducing forces and displacements from both mechanical and thermal sources.

Failure mechanisms to be considered in design for high temperature include the following.

Firstly, failure mechanisms encountered in low temperature applications include:

i. Limit load collapse, under a single load application.

ii. Excessive displacement and/or deformation, limiting functionality, under a single load application, below the limit load.

iii. Structural instability or buckling, under a single load application.

iv. Progressive collapse by ratcheting under cyclic load.

v. Fracture by the initiation and/or propagation of a crack under a single load application.

vi. Fatigue failure under cyclic loading.

vii. Breach of the pressure boundary, or structural collapse caused by corrosion induced loss of section.

In addition to these, failure mechanisms specific to high temperature operation include,

viii. Creep rupture - loss of pressure boundary integrity due to a) the formation of local cracks, or b) general limit load collapse, due to creep induced continuum damage under essentially steady load.

ix. Excessive deformation - loss of functionality, due to creep deformation under essentially steady load. 
x. Creep buckling - time dependent structural instability leading to catastrophic collapse or loss of function

xi. Cyclically enhanced creep deformation - Accelerated creep deformation caused by repeated resetting of stresses by cyclic plastic strain, due to cyclic loads superimposed on a sustained load history. Also referred to as “creep ratcheting”.

xii. Accelerated creep rupture - Accelerated creep damage caused by repeated resetting of stresses by cyclic plastic strain, due to cyclic loads superimposed on a sustained load history.

xiii. Creep/fatigue interaction - Failure under cyclic conditions in a period, usually less than fatigue due to the cyclic condition alone, or creep rupture due to time-at-stress alone, the mechanism for which may include other time/temperature related phenomena, such as oxide layer cracking, and may be material specific.

Finally, modifications to material properties may be influenced and suffer deterioration due to the following at all temperatures.

xiv. Ageing induced by temperature, strain, radiation, or diffusion leading to modification in any or all of the phenomena addressed in items i) through xiii) above.

xv. Corrosion, oxidation, and mass transfer phenomena.

xvi. Irradiation induced failure mechanisms.

\section{DESIGN CRITERIA}

An acceptable design is one which has a demonstrably acceptable resistance to the loadings listed in 2 above. This is done conventionally by comparing performance parameters of the component, based on understanding of its operating conditions, with allowable limits usually, but not always, based on material properties. An exception to 
this rule is, for instance, functional limitation due to excess deformation. In complex applications, such as those involving nonlinear or time dependent behavior, design criteria may call for consideration of both material properties and geometrical factors simultaneously as, for instance, in the use of limit load concepts in evaluating primary load carrying capability.

A major feature of all mechanical design Codes is a recognition that "stress" is not a sufficient basis for a failure criterion. Depending on the nature of the failure mechanism involved, the appropriate criterion may be only part of the total stress, or a function of the multiaxial stress state. Methods of stress classification are therefore an element which is present in all reputable Codes. A review of the structural concepts underlying the development of both design criteria and design evaluation methodologies is reserved for reporting in a later subtask.

For current purposes, it is useful to refer frequently to one important classification, which is the distinction between "primary" stress, denoting that part of the total stress in equilibrium with external mechanical forces, and "secondary”, which consists of all contributions to an internal, self equilibrating or residual stress state. The former is instrumental in causing gross structural collapse whereas the latter is only of concern in situations of cyclic load or local damage accumulation.

With this qualification in mind, the following criteria correspond approximately to the load cases listed in the previous section.

i. Limit Load - A minimum requirement of any component is that it be able to support a single application of the worst combination of all the static loads to which it is subjected. This may be achieved conservatively by limiting service stresses or, in components constructed from ductile materials, by ensuring that the collapse load exceeds the maximum service load by a suitable factor. Establishment of a limit load may be by analysis or experiment.

ii. Limiting excessive deformation - Any number of functional and nonfunctional considerations may require a limit to be placed on displacement and/or deformation, even when the deformations are 
"small”, i.e. insufficient to affect structural integrity by altering the load carrying capacity of the component. A deformation or strain limit may be defined in the Code as a fixed \% of a characteristic dimension, or local strain, or may be left to the user to determine as a contractual matter based, for example, on some functional requirement. Note, displacement may be elastic, while deformation is caused by inelasticity.

iii. Buckling - The influence of initial and/or load induced geometric imperfections may lead to premature local or gross structural collapse. Both classical buckling of the Euler instability type, or by amplification of initial geometric features need to be considered. Assurance against buckling is based on material strength and stiffness properties combined with geometry and may be evaluated by geometry-specific buckling load based on detailed or conservative approximate calculations or testing.

iv. Ratcheting - Incremental collapse under a cyclic sequence of loads needs to be avoided. Avoidance of this state is a function of cyclic plastic yield. Avoidance of ratcheting can be assured by demonstrating shakedown to a stable cyclic elastic state using a conservative approximate method, or by detailed cyclic analysis, the latter most likely based on FEA.

v. Fast Fracture - Failure by cracking rather than gross plastic deformation is a high consequence risk because it depends on local conditions rather than gross structural behavior and is often without warning and catastrophic. The first line of defense is often material selection based on measures of fracture resistance which may be either empirical, such as CVN data, or mechanics based such as fracture toughness. Since cracks should not be present, although they often are as a practical matter, fracture is frequently not considered explicitly as a design criterion, it being assumed that the problem has been avoided by material selection, combined with appropriate surveillance of the manufacturing process. A "postulated crack" is used in some post construction evaluations such as that provided 
by Section XI of the ASME Code. This approach could be used in firsttime design as well.

vi. Fatigue - Crack initiation and propagation may be a precursor to fast fracture, to limit load collapse by yielding of a remaining ligament, leaking or distortion. It is a function of local stress/strain state rather than gross structural behavior. The current form of evaluation of fatigue life uses either stress/life or strain/life data as the design criterion. Evaluation is material specific information but some general rules, such as Manson’s Universal Slopes or the traditional “endurance limit” concept must often serve as substitutes in preliminary design due to the lack of specific data.

vii. Corrosion - Corrosion alone is invariably considered as a material selection process preceding design, except for the specification of corrosion loss allowances for use in determining net section sizes.

Elevated temperature operation introduces thermally activated, time dependent processes with the following associated design considerations. Creep is the foremost of these. Creep causes both time dependent deformation and changes to the material which may be damaging, referred to as “continuum damage”. Continuum damage results in both loss of strength and of ductility leading to either a time dependent collapse or formation of a crack, or both.

viii. Creep rupture - Creep rupture failure is the time dependent equivalent of limit load collapse at low temperature where material strength, in the form of a factored yield or ultimate tensile strength is replaced by the creep strength of a standard specimen for some specified finite life. This may be a single representative life, such as the $2 / 3^{\text {rds }}$ stress for rupture in 100,000 hours adopted by Sections I and VIII of the ASME Code, or a function of time, as defined in API 530 for heater tubes, and Section III/NH for nuclear components. As currently applied, this criterion invariably does not distinguish between specimen creep failure as being due to ductile instability or void coalescence and growth, although the question of creep ductility may be considered implicitly in the material selection process. As 
currently applied by most Codes in use, this criterion includes two very different failure mechanisms, a general collapse due to propagating continuum damage, and local cracking due to ductility exhaustion at points of high stress.

ix. Excessive creep deformation - Identical to ii above, but including time dependent creep deformations based on the design life and load history. This criterion is aimed at general structural distortion, but is often applied as a limit on a local strain measure, not necessarily the local maximum strain.

x. Creep buckling - Repeat of iii, including time dependent deformations in the form of either demonstrably conservative approximate methods, or by detailed FEA.

xi. Cyclically enhanced creep deformation - Demonstration that reversed plastic straining due to cyclic loads does not lead to an accelerated general creep deformation rate at a structural level. Alternatively, demonstration that such accelerated deformation, if it occurs, does not exceed the functional limits of the component as defined for creep under nominally steady loading conditions. Creep deformation and strain limits remain the same as for nominally constant load, but account for the constantly transient state of stress due to the resetting process.

xii. Accelerated creep rupture - Demonstration that accelerated creep damage due to periodic resetting of stresses due to cyclic plasticity, if it occurs, does not exceed Code design criteria for cumulative damage.

xiii. Creep/Fatigue interaction - Demonstration, by some Code accepted procedure that material damage due to the combined effects of cyclic loading and sustained hold periods at elevated temperature do not exceed allowable limits.

xiv. Ageing effects - The effects of ageing are found in changes to material properties already considered as part of design criteria described earlier. 
The quantitative effects are material, manufacture and operationally specific and can only be evaluated by purpose designed test programs.

xv. Corrosion, oxidation, and mass transport phenomena - demonstrations of how these effects take into account effective section size, and possible material interaction or degradation.

xvi. Radiation effects - demonstration and incorporation of material degradation or changes, including possibly drastic changes in applicability of failure criteria, e.g. ductile vs. brittle failure criteria. Some examples of radiation effects include but are not limited to:

- Irradiation induced swelling

- Irradiation induced creep

- Plastic Flow localization due to irradiation (dislocation tunneling)

- Ductility exhaustion due to irradiation exposure

- Irradiation induced effects on fatigue, and on C-F

- Seismic loads - demonstration and assurance that such severe and infrequent loading, or more frequent depending upon location, does not pose as a safety risk to the public, and criteria to define permissible operation after such an event, or not.

\section{DESIGN EVALUATION}

Assurance of structural integrity is achieved by a combination of 3 activities, component analysis, material and component testing, and surveillance, results of which are to be compared with allowable limits of material, or combined material, geometric performance.

In order to make this comparison, it is necessary to take into account the fact that the raw results of stress, strain and deformation are, in general, inadequate as design 
criteria and need to be grouped into meaningful classifications, such as "primary", "secondary", etc., for the purpose. This process is subject to much interpretation and requires clear guidance from the Code in order to ensure consistent and repeatable results.

A systematic procedure for the classification of stress - or any other parameters used in design evaluation - is a necessary adjunct to any suite of analytical or other methods of component evaluation.

Techniques for design evaluation are, by their very nature, approximate. A comprehensive assessment therefore takes the form of a number of submodels of the entire system, each with a limited objective, such as determination of gross structural collapse, or local cyclic stress/strain histories for the purpose of estimating material damage of various forms.

\subsection{Analysis}

Design evaluation invariably requires two levels of analysis.

Level 1 is required in the initial design stages, when scantling sizes and material selection is carried out for the first time.

Level 2 involves more detailed analysis aimed at ensuring that preliminary design decisions were essentially correct, to fine tune dimensions and to verify compliance of the design with respect to the more complex failure mechanisms such as cyclically induced failures or structural instability.

Each such submodel has generated its own suite of simplified methods of analysis with varied degrees of suitability, depending on the circumstances, including prior knowledge of the load histogram, material properties and resources available to the designer. Specific methods of approximate analysis are addressed in later subtasks of Task 9.3. It is sufficient for present purposes to list the commonly identified submodels.

1. Limit load models. Used to determine the resistance of the component to gross structural collapse under both short term and long term loading 
2. Shakedown or Ratcheting models. Aimed at determining the overall dimensional stability of components under cyclic conditions, i.e. avoidance of incremental collapse. Using the term in a broad sense, “shakedown” models are intended to deal approximately with both short term incremental failure by plasticity alone, and with combinations of plastic and creep deformations due to on/off loading which includes hold times at temperature.

3. Local Plasticity models. Aimed at approximating local, inelastic behavior in regions of constrained plastic deformation, as in notches or surface effects during severe thermal transients. The phenomenon of local constrained inelastic deformation is commonly referred to as "elastic follow-up”. Elastic follow-up analysis avoids detailed inelastic analysis of an entire structure in order to establish the local stress/strain histogram in local regions where fatigue, accelerated creep damage or creep/fatigue interaction may occur.

\subsubsection{Level 1 Fundamental Design}

Compliance with Level 1 is achieved by demonstrating the compliance with respect to certain critical failure modes such as gross limit collapse, based on allowable stresses or other fundamental material properties derived from testing, using simplified methods of analysis selected on the basis of a reasonable degree of consistent conservatism. Level 1 corresponds roughly to those sections of the ASME Code currently designated as "Design by Rule”, and exemplified by the use of "hand calculations" although this expression is intended to characterize the level of simplicity involved rather than the actual method of implementation.

Alternatively, compliance with Level 1 may require a priority in satisfying other failure modes, such as creep-fatigue or ratcheting, rather than primary limit loads. One example application may be the design of Liquid Metal Fast Breeder Reactors.

In simple applications, it should be feasible to carry out all necessary design work at Level 1. In elevated temperature applications, especially where cyclic loading is concerned, a Code may be overly conservative or even impossible to achieve. In such 
cases, it may be necessary to resort to more detailed methods. Typically, the more detailed methods may be grouped or found in the Level 2 category.

In keeping with the intent to guide preliminary design decisions, the information required as regards material properties is minimal.

\subsubsection{Level 2 Detailed Design}

Detailed design at this level is generally brought into play for any or all of the following reasons.

i. The complexity of material response exceeds the level which can be adequately captured with simple design allowables. For example, detailed constitutive modeling of creep deformation behavior is required in place of a simple allowable stress for rupture in a specified time.

ii. Loading, including temperature variations, exceeds the complexity that can be adequately dealt with using minimal material properties.

iii. Final validation of a completed design under realistically simulated operating conditions.

The 'Ideal' Code would be expected to contain specific guidelines for the analysis of common components, especially at Level 1 . In certain critical instances these guidelines may be designated as mandatory if a significant safety issue is at stake. The 'Ideal' Code would also provide the opportunity to employ alternative means of design analysis contingent on such alternatives passing a validation process to be defined and administered by the relevant Code body.

A relatively recent development that needs to be considered (i.e. within the past 2 decades) in the formulation of any design Code today is the universal adoption of computer based methods of structural analysis. At one time serious objections to the adoption of computer based methods such as FEA were the cost, and education in their use. These objections are now largely obsolete. In fact it is now quicker and easier to use such methods than to attempt more traditional "pencil-and-paper" evaluations, because the essential facilities for their use are as common in most engineering offices as the 
copier. This does not mean that there are no longer problems associated with the use of computer based methods in design however.

Unlike earlier methods which were based on analytical expressions and, sometimes, empirical rules derived entirely by experiment, a computer based solution is invariably highly geometry specific, and it is extremely difficult to apply standards which relate directly to a given structural shape. In fact, the ability to construct geometric models which mimic the shape of the actual structure with fine precision is one of the chief attractions of computer based design.

Consideration needs to be given in the Code to how such computer based methods are to be validated before use in critical applications and how the validation process should be administered without being excessively restrictive or time consuming.

\subsection{Testing}

Testing covers two categories,

a. material testing for the purpose of developing allowable stress and other parameters for design purposes, and

b. component testing as an alternative to analysis as a means of design validation.

The latter, while more complex to implement, is relatively simple to define. It is an alternative method of design validation which is, or should be, acceptable, subject to the requirement that it follows a process which can be reviewed and validated by the Code body. In this respect it is in all ways similar to the use of computer based methods of assessment.

Material testing in the modern age requires some updating.

In the past, design was dictated by simple methods which, in turn, called only for relatively simple material properties. In addition, it has been the practice for material science and mechanics, i.e. the generators of material information, and the users of same, to operate increasingly in mutually separate domains. As long as the interface was a 
simple one, such as the need for Young's Modulus, and yield stress, the split was not a critical concern.

Design for elevated temperature has developed for many years whilst attempting to retain this simplifying division of labor as long as possible. With the movement to design for increasingly stringent conditions, calling on more complex measures of material behavior, simplistic measures of material strength are no longer sufficient as the input for the more detailed evaluations increasingly being considered a minimum requirement for high temperature evaluation. This issue is considered in more depth later in this report.

\subsection{Surveillance}

Surveillance, or in-service inspection of components with finite life expectancy, is advisable, if not necessary, to ensure continued structural integrity. Currently, in the USA at least, initial design and fitness for continued service (FFS) are considered as distinct entities, covered by separate parts of the ASME Code as a whole. Without venturing to debate the merits or otherwise of this approach, increasingly severe operating conditions place a heavy responsibility on the design process to predict, ab initio, the entire lifelong performance of a component, whose behavior is critically dependent on several factors which may not be completely predictable by present day methods. Uncertainties which can be easily identified are,

i. Uncertainty over long term material properties. In most instances, understanding of the high temperature long term properties of materials is imperfect at best, and such information as exists is indirect, having been derived from extrapolations based on short term testing.

ii. Plant operational parameters can only be estimated at the design stage and may be either grossly over- or underestimated compared with actual service conditions. Given that elevated temperature performance is a highly nonlinear function of both mechanical and thermal loadings, small changes have large consequences. 
Other contributions add to the need for some form of ongoing surveillance to be incorporated into the design of high temperature equipment. While it may not be the place of the designer to specify such surveillance, it should be a part of the design to accommodate the needs of surveillance. Precedence exists for this concept in isolated cases. For instance, the ban on the use of fillet welds in pressure boundaries is generated by the inability to inspect the root pass.

\section{MATERIAL PROPERTIES REQUIRED TO PERFORM A DESIGN ASSESSMENT}

Material properties required for design purposes are listed below. These are not proposed as an exhaustive listing, but merely represent the current standard state-of-theart available to industry at large.

A. For Short Term v temperature
i. Young's modulus
ii. Yield Stress
iii. UTS
iv. Ductility
v. Fracture toughness
vi. Thermal expansion
vii. Thermal conductivity
viii. Thermal diffusivity

B. Long Term (Level 1) vs. time and temperature
i. Rupture strength
ii. Creep deformation
iii. Fatigue
iv. Creep/fatigue interaction

C. Long Term (Level 2) vs. time and temperature

i. Creep constitutive model

ii. Creep ductility as function of stress state 
iii. Cyclic constitutive behavior, including cyclic stress/strain and creep/plasticity interactions (e.g. cyclic softening)

\section{Oxidation/Corrosion Resistance}

E. Irradiation Effects

\section{DOCUMENTATION}

A comprehensive specification for documentation is necessary, both of the Code itself to ensure that there is no ambiguity in interpretation or application of intent or requirements, and for users, to provide clear demonstration of compliance.

In the case of the Code, the following elements are essential

i. A declaration of Code scope, responsibilities and limitations

ii. A statement of user responsibilities and constraints

iii. A mechanism for soliciting and implementing changes

iv. A system for incorporating modifications, publishing updates and documenting revisions

v. Instructions to users on documentation required to be submitted in support of a design in order to show compliance with the Code

vi. Delineation between mandatory procedures, if any, and optional procedures to be offered as guidelines and rules for validating users defined procedures where these are permitted.

vii. Wherever the user is deemed ultimately responsible for the accuracy and correctness of any design procedures, including procedures mandated by the Code, reasonable access should be provided to the sources of information used in developing these procedures, so that users can perform independent checks, e.g. references.

In the case of the user, 
i. Any design according to the Code should be supported by documentation demonstrating compliance with Code requirements in sufficient detail to enable an independent review to follow the design process in full.

ii. Any technical application should be traceable to validated sources of information, including but not limited to published literature, Code committee proceedings or fully documented reports of independent studies.

iii. In the case of methods such as computer Codes, evidence should be made available to verify the accuracy and precision of the Code used, both as a general tool, and in the specific application by demonstration, for example, by comparison with problems having known solutions.

\section{CONCLUSION}

1. This report describes work conducted toward developing a template for what might be the "Ideal" high temperature design Code.

2. While attempting to be as comprehensive as possible as to subject matter, it does not presume to recommend what individual components of a Code should be implemented, some of which is the focus of other Tasks in the DOE-ASME Gen IV / NGNP Materials Projects.

3. This report does serve as a basis for construction of an attribute chart which is being prepared as part of Task 9.2; the intention for which is to provide a uniform format and concise means for summarizing and comparing other high temperature Codes currently in use around the world. 


\title{
Task 9: Update and Improve Subsection NH - Simplified Elastic and Inelastic Design Analysis Methods
}

\section{Subtask 9.2 - Review of International ETD Codes}

Gen IV / NGNP Materials Project

\author{
Submitted to \\ Mr. Jim Ramirez \\ Vice President, Business Development \\ ASME Standards Technology, LLC \\ Three Park Avenue \\ New York, NY 10016 USA
}

\begin{abstract}
By
Peoria, IL

On behalf of

Jeries Abou-Hanna

Douglas L. Marriott

Timothy E. McGreevy
\end{abstract}

Advanced Consulting Engineering Services, Inc.

October $30^{\text {th }}, 2008$ 


\section{TABLE OF CONTENTS}

\begin{tabular}{|c|c|}
\hline .2 .1 & \\
\hline 9.2 .2 & 5 \\
\hline 9.2.2.1 & R5 - High Temperature .... \\
\hline 9.2.2.2 & esign Loads \\
\hline 9.2.2.3 & ailure Mechanisms .. \\
\hline 9.2.2.4 & esign Criteri \\
\hline .2 .3 & Ionju . \\
\hline 9.2.3.1 & 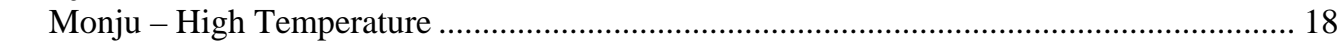 \\
\hline 9.2.3.2 & ts \\
\hline 9.2.3.3 & ailure Mechanisms …………......................... \\
\hline 9.2.3.4 & riteria / Procedures .... \\
\hline 9.2.3.5 & 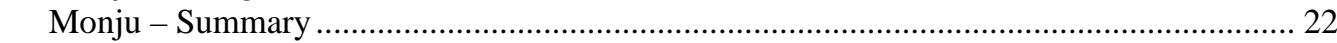 \\
\hline 2.4 & ……................................... 37 \\
\hline 9.2.4.1 & rature \\
\hline 9.2.4.2 & 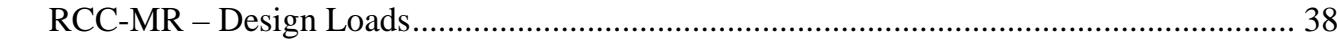 \\
\hline 9.2.4.3 & 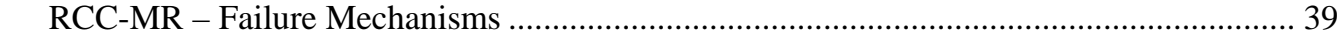 \\
\hline 9.2.4.4 & 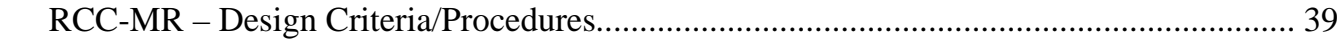 \\
\hline 9.2 .5 & (1) \\
\hline 9.2.5.1 & …........................... 52 \\
\hline 9.2.5.2 & ……………………........... 53 \\
\hline 9.2.5.3 & 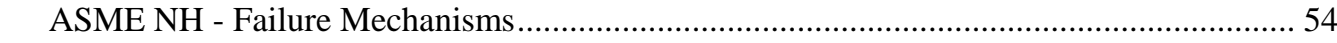 \\
\hline 9.2.5.4 & ia / Procedures... \\
\hline .2 .6 & PI579 \\
\hline 9.2.6.1 & ature \\
\hline 9.2.6.2 & 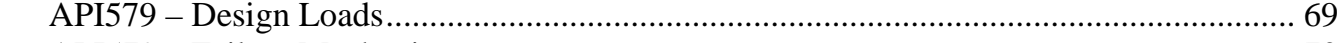 \\
\hline 9.2 .6 .3 & echanisms .. \\
\hline & \\
\hline 2.7 & ......86 86 \\
\hline
\end{tabular}




\section{LIST OF TABLES}

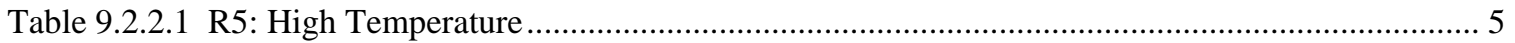

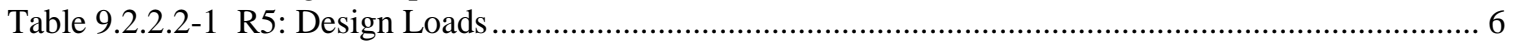

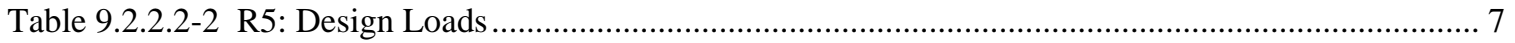

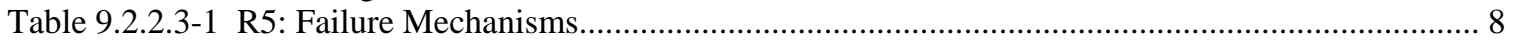

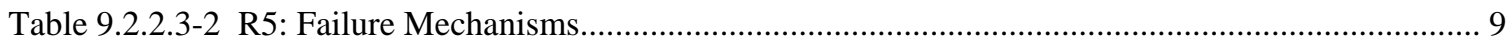

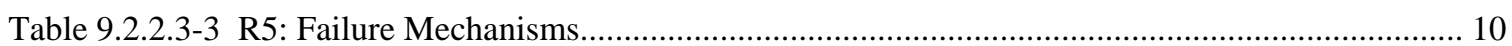

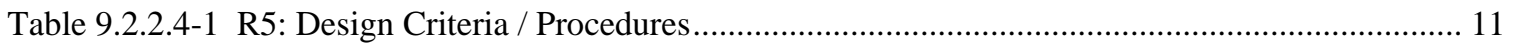

Table 9.2.2.4-2 R5: Design Criteria / Procedures.................................................................................... 12

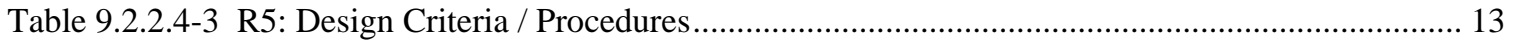

Table 9.2.2.4-4 R5: Design Criteria / Procedures...................................................................................... 14

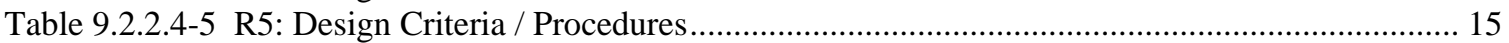

Table 9.2.2.4-6 R5: Design Criteria / Procedures........................................................................................ 16

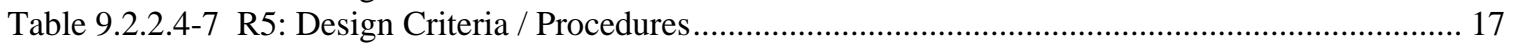

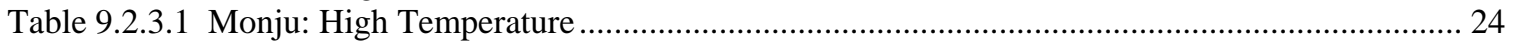

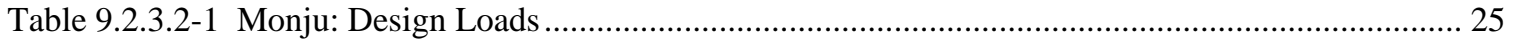

Table 9.2.3.2-2 Monju: Design Loads ............................................................................................... 26

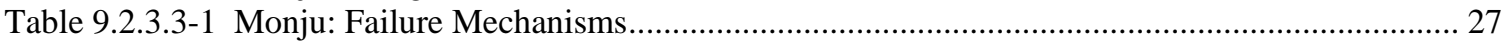

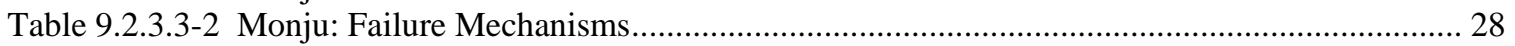

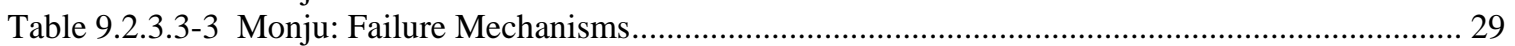

Table 9.2.3.4-1 Monju: Design Criteria / Procedures..................................................................................... 30

Table 9.2.3.4-2 Monju: Design Criteria / Procedures.................................................................................... 31

Table 9.2.3.4-3 Monju: Design Criteria / Procedures ................................................................................... 32

Table 9.2.3.4-4 Monju: Design Criteria / Procedures ................................................................................... 33

Table 9.2.3.4-5 Monju: Design Criteria / Procedures..................................................................................... 34

Table 9.2.3.4-6 Monju: Design Criteria / Procedures ................................................................................. 35

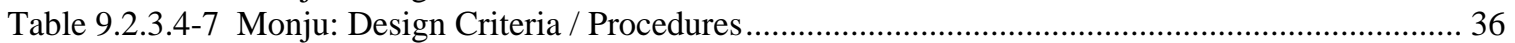

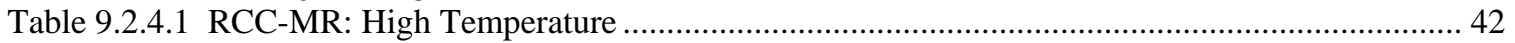

Table 9.2.4.2-1 RCC-MR Procedure: Design Loads ................................................................................. 42

Table 9.2.4.2-2 RCC-MR Procedure: Design Loads ................................................................................ 43

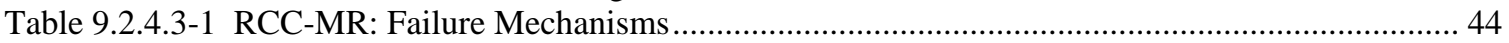

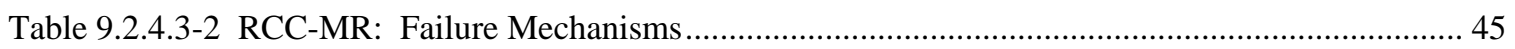

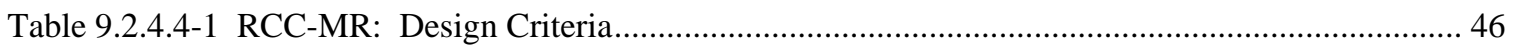

Table 9.2.4.4-2 RCC-MR: Design Criteria......................................................................................... 47

Table 9.2.4.4-3 RCC-MR: Design Criteria............................................................................................ 48

Table 9.2.4.4-4 RCC-MR: Design Criteria.......................................................................................... 49

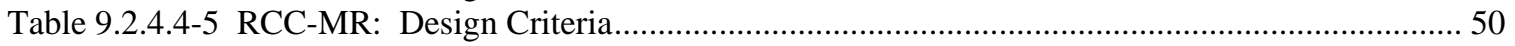

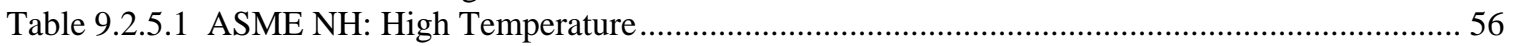

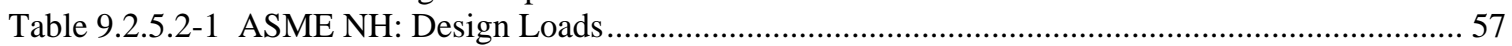

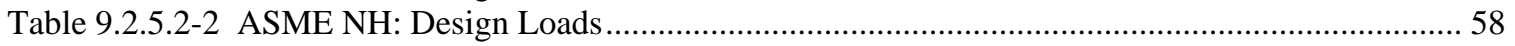

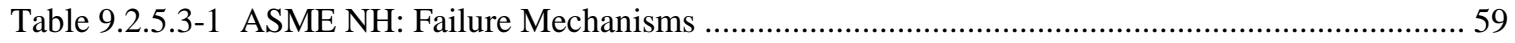

Table 9.2.5.3-2 ASME NH: Failure Mechanisms ………......................................................................... 60

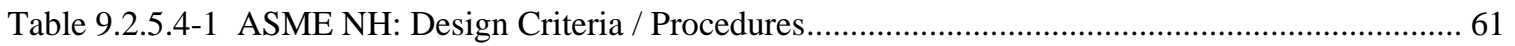

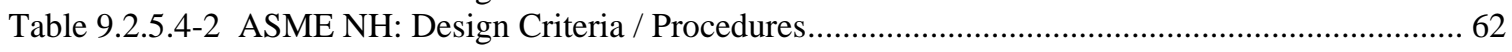

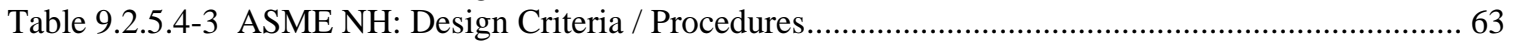

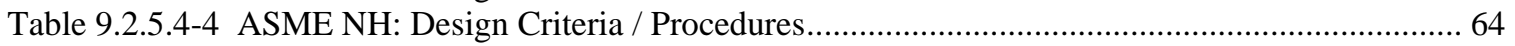

Table 9.2.5.4-5 ASME NH: Design Criteria / Procedures............................................................................. 65

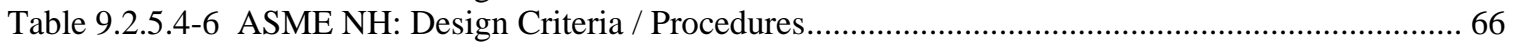

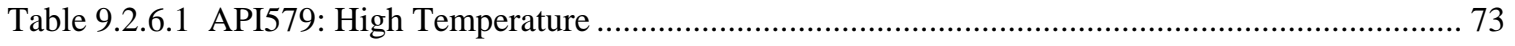

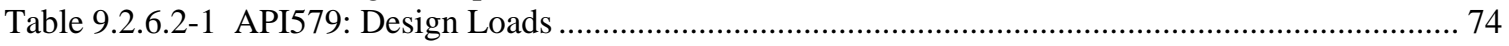

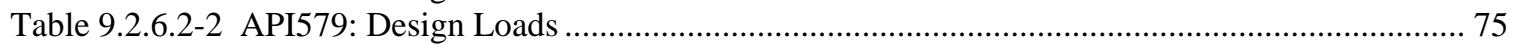

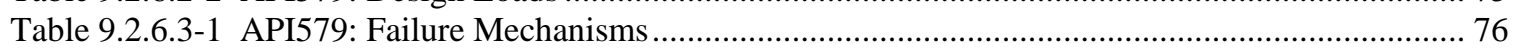

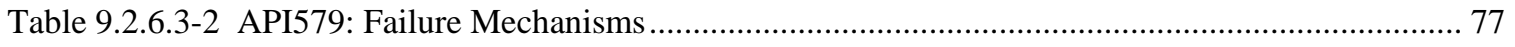

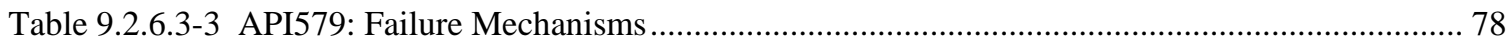

Table 9.2.6.4-1 API579: Design Criteria / Procedures ................................................................................. 79 


\section{LIST OF TABLES}

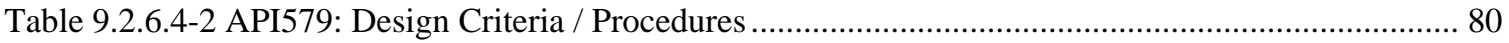

Table 9.2.6.4-3 API579: Design Criteria / Procedures ..................................................................... 81

Table 9.2.6.4-4 API579: Design Criteria / Procedures .......................................................................... 82

Table 9.2.6.4-5 API579: Design Criteria / Procedures ........................................................................... 83

Table 9.2.6.4-6 API579: Design Criteria / Procedures .............................................................................. 84

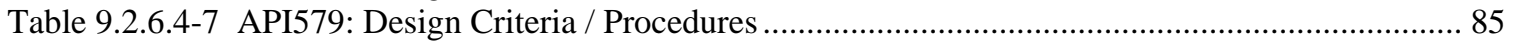




\section{LIST OF FIGURES}

Figure 9.2.5.1 Timeline of ASME NH development..................................................................................... 52

Figure 9.2.5.2 Post 1980 U.S. ETD R\&D limited relative to that internationally ....................................... 52 


\subsubsection{SUBTASK OBJECTIVE}

The objective of this subtask is to review International ETD Codes, e.g. R5, Monju Code (JNC), RCC-MR, API 579, and ASME NH, providing a broad summary of the scope of each code, standard and/or guideline, in comparison to the baseline requirements of the 'ideal' ETD Code as defined in Subtask 9.1. Specifically,

i. Identify limitations, such as failure mechanism omitted or inadequately catered for,

ii. Identify methods mandated, and material data required for mandated methods,

iii. Identify recommended but optional methods, and material data required for optional methods,

iv. Identify if freedom is given to designers/operators to use unspecified procedures and safeguards, and if any recommendations, criteria, or means are provided to ensure that such methods and their use are appropriate and/or validated.

The reviews were conducted and presented in a consolidated manner in order to facilitate understanding of each; the intent is also to provide a guide to each Code for comparison to one another, both in Subtask 9.3 (limited in scope according to Task 9), as well as future possible needs for ASME, DOE, and particularly the ASME SG-ETD. Highly relevant information for each International Code of interest relative to the Ideal ETD Code summarized in Task 9.1 was summarized in four types of tables: 1) High Temperature, 2) Design Loads, 3) Failure Mechanisms, and 4) Design Criteria/Procedures. The information and tables are presented and summarized for each individual International Code. No attempts were made to compare the Codes with one another, only relative to the Ideal ETD Code, as such comparisons are readily identified as out of scope for Task 9.

\subsubsection{R5}

The 'R5 Procedures' are assessment procedures for the high temperature response of structures. The assessment procedures are maintained by the R5 Panel under a Structural Integrity Assessment Procedures collaboration involving British Energy, Rolls-Royce, and Serco Assurance. Unlike many other International Codes, R5 is a 'Fitness for Service' procedure. Furthermore, the procedures involve assessment of initially defect free components and the growth of such flaws by creep and creep-fatigue mechanisms. This review was conducted using R5 Issue 3, released in July 2003, which supersedes all earlier issues.

In short, many of the basic principals by which elevated temperature design throughout the world are based were taken from ASME's SG-ETD, of which the most relevant Code book is ASME-NH. While a direct comparison of R5 to ASME-NH is out of scope, it is worth noting that the 'Foreward' of R5 clearly states that 'R5 is ... intended to augment and replace, where necessary, the provisions of ASME III Subsection NH and the French 
Code RCC-MR.” This statement is well supported by the fact that the United Kingdom continued $\mathrm{R} \& \mathrm{D}$ in the area of high temperature structural materials and design over the last 30 years, whereas limited efforts were supported in the United States after the Three Mile Island accident in 1979 and the subsequent demise of new nuclear power plants in the United States.

R5 is comprised of seven (7) volumes: 1) Overview, 2/3) Creep-Fatigue Crack Initiation Procedures for Defect-Free Structures, 4/5) Procedure for Assessing Defects Under Creep and Creep-Fatigue Loading, 6) Assessment Procedure for Dissimilar Welds, and 7) Behavior of Similar Weldments - Guidance for Steady Creep Loading of Ferritic Pipework Components. Efforts were focused on Volume 2/3, as it ultimately relates directly to ASME-NH and the interests of GENIV/NGNP.

\subsubsection{R5 - High Temperature}

Two main definitions / criterion were identified in the Ideal ETD Code: a High Temperature Limit, and a Criterion for Insignificant Creep. Table 9.2.2.1 below summarizes this information. The 'High temperature limit' is summarized in ID\# 'a', the definition is 'covered' by R5, the appropriate location in the Code is indicated, as well as the inclusion of appropriate comments. Specifically, the creep rupture curves are provided as a function of time and temperature for various permissible materials. The criterion used to establish such curves is also indicated for future reference. Note, R5 refers to a consolidated body of material data found in R66; R66 was not available for purchase by the authors, and is restricted in use and distribution. However, note that R5 often refers to other sources of data, including ASME-NH, RCC-MR, and NIMS as suitable resources.

Similarly, the insignificant creep definition is covered, with details regarding the definition summarized in the comment section. Note, $\mathrm{T}_{\text {ref }}$ is the reference temperature, which corresponds to the reference stress used in the Code. Also, numerous Appendices are contained in each Volume and are indicated for example by A1.6 for the insignificant creep criterion. Appendices exists for each Volume, and their numbering starts at 1 for each, i.e. Volume 2/3 has an Appendix A1, and Volume 4/5 has an Appendix A1. However, most if not all references to Appendices are to Volume 2/3; Volume 4/5 was not covered in detail, as it is specifically being addressed by another DOE-ASME Task.

\subsubsection{R5 - Design Loads}

Table 9.2.2.2-1 and 9.2.2.2-2 summarize information relevant to 'Design Loads' for an Ideal ETD Code, including the Definition / Criterion, whether the Definition is 'Covered', the location in the Code where the Definition is addressed, and relevant 
comments. Of particular interest are the following:

- Significant guidance is given for development of a load histogram and cycle definitions where the cycle of interest is the steady cyclic state; while noted in general, the transient effects from one cycle to another - or early behavior in route to establishment of the cyclic state - are assumed to be negligible.

- The fundamental use and understanding of primary and secondary stresses, membrane, local, bending, and peak stresses are utilized, although as will be discussed later, design criteria and procedures permit use of reference stress approaches which do not require stress linearization and categorization.

- Since R5 is an assessment Code, differentiation of load categories is not made. However, two important aspects are:

o structural behavior is limited to elastic, shakedown, or plasticity; loading into the ratcheting regime is not permitted, unless the assessment is made with inelastic analysis, e.g. inelastic finite element analysis.

o Interaction with severe dynamic loading, e.g. earthquakes, is not covered, and requires inelastic analysis.

\subsubsection{R5 - Failure Mechanisms}

R5 is very well organized. Specifically, to ensure against specific failure mechanisms a clear link between procedure, criteria, and the mechanism being addressed is made. This is accomplished by presenting the contents in a step by step fashion consistent with the flow charts / diagrams provided. Sections of the Code summarize the intent and meaning of the step / procedure, and are often summarized in general terms that lend satisfaction of the step / procedure with any justifiable engineering solution / method that meets the intent. Often a simple means of satisfying the intent of the step / procedure is given, with reference being made to one or more of the Appendices for further details and other optional approaches. Also, many references are included throughout the Code to internal reports, open literature, etc. for the interested engineer, and perhaps more importantly for future Code developers and regulation bodies, as needed. The method of organization minimizes the extent of redundant and/or scattered information, rendering the reading, ease of use / implementation, and interpretation of the Code quite easy.

Tables 9.2.2.3-1 thru 9.2.2.3-3 summarize the extent by which failure mechanisms of an Ideal ETD Code are addressed by the R5 procedure. The information is summarized in the same manner as Tables 9.2.2.2-1 and 9.2.2.2-2. Three possibilities exist for response to the column labeled 'Covered?', “Yes”, “No”, and "Yes/No”, with the latter requiring an explanation. "Yes/No" indicates that the Code does not explicitly address the failure mechanism or does in a limited way or circumstances; but the Code does address the mechanism indirectly to some extent. For example, 'Excessive deformation limiting functionality, under a single load application' is indirectly covered by safety factors on Sy for limit loads. 
R5 does not address corrosion, mass transfer phenomenon, etc, creep buckling, softening enhanced cyclic creep rupture, irradiation effects, and limited attention to thermal aging effects.

\subsubsection{R5 - Design Criteria/Procedures}

Tables 9.2.2.4-1 through 9.2.2.4-7 summarize the various design criteria of an Ideal ETD Code and how R5 addresses them or not. The first column indicates the design criteria, and the failure mechanism addressed by the criterion is listed in the second column; whether or not the R5 Procedure covers the design criteria is indicated in the third column. Limitations of the criteria, justification \& explanation / references for the design criteria, and recommended procedures are also listed, including whether or not they are mandatory or not. Alternative procedures are summarized, including the locations in the Code that address the criteria. Finally, comments are included as appropriate.

Of particular relevance is the option to use conventional stress linearization procedures; however, while such conventional approaches are permitted, R5 emphasizes and utilizes the reference stress approach for both monotonic and cyclic applications. Regardless of the approach used, unless full inelastic analysis is conducted, for which R5 provides significant guidance, structural behavior is limited to elastic, shakedown (global shakedown), or plastic (shakedown for $80 \%$ of a section or ligament); assessment of structures within the ratcheting regime is not covered. Cyclically stable yield strengths are utilized for assessment of cyclic reference stress.

Creep ductile and brittle materials are addressed by modification of the reference stress; the linear damage rule is utilized for C-F interaction, although creep damage is based upon a ductility exhaustion approach. Elastic follow-up is utilized in determination of strain range for C-F analysis. No design criteria are provided to assess radiation effects and associated failure mechanisms. Stress concentrations greater than four (4) are treated as pre-existing defects, i.e. no nucleation life exists. Thermal aging effects are limited to fatigue strength reduction factors. 
Table 9.2.2.1 R5: High Temperature

\begin{tabular}{|c|l|l|l|l|}
\hline ID\# & Definition / Criterion & Covered? & Location & \multicolumn{1}{|c|}{ Comment } \\
\hline a & High temperature limit & Yes & $\begin{array}{l}\text { Volumne 2/3 Section 3.7, } \\
\text { R66. }\end{array}$ & $\begin{array}{l}\text { S }_{\mathrm{R}} \text { : 'creep rupture' curves as function of time and temperature } \\
\text { provided for permissible materials, defined as lower of a) } \\
\text { appropriately defined lower bound rupture stress (e.g. mean } \\
\text { rupture stress divided by 1.3), and b) average stress to 1\% } \\
\text { creep strain for ferritics, 2\% creep strain for austenitics. }\end{array}$ \\
\hline b & $\begin{array}{c}\text { Criterion or definition of } \\
\text { temperature limit for } \\
\text { insignificant creep effects }\end{array}$ & Yes & $\begin{array}{l}\text { Volumne 2/3 and 4/5 } \\
\text { V2/3: Section 5.6, 6.4, A1.6 }\end{array}$ & $\begin{array}{l}\text { Time fraction <1 relative to insignificant creep curves. } \\
\text { Curves developed based upon 1 of 2 criteria: } \\
\text { a) time and temperature required for 20\% stress relaxation } \\
\text { from 1.35Sy (constant strain) - material in cyclic steady state, } \\
\text { or b) 0.03\% creep strain at constant stress of 1.25Sy. } \\
\text { (Sy==min0.2\%Proof Stress). *Note, based upon T=Tref, or } \\
\text { conservatively Tmax. }\end{array}$ \\
\hline
\end{tabular}


Table 9.2.2.2-1 R5: Design Loads

\begin{tabular}{|c|c|c|c|c|}
\hline ID\# & Definition / Criterion & Covered? & Location & Comment \\
\hline a & $\begin{array}{l}\text { Guidelines for load historgram \& } \\
\text { cycle definition }\end{array}$ & $\begin{array}{c}\text { General } \\
\text { Disclaimer }\end{array}$ & $\begin{array}{l}\text { Vol 2/3 Section 6.1. } \\
\text { Appendices A2, A3, A4; } \\
\text { Appendix A12 }\end{array}$ & $\begin{array}{l}\text { Guidance on various simplification and construction of load cycles is provided; } \\
\text { transition effects from early cycle behavior to steady cylcic state is noted with } \\
\text { special attention provided in Appendix } 4 \text { - mainly for residual stresses in } \\
\text { weldments, but applicable in general. } \\
\text { Advice on inelastic analysis is provided. }\end{array}$ \\
\hline $\mathrm{b}$ & Glossary of terms & Yes & $\begin{array}{l}\text { Nomenclature at start of each } \\
\text { Volume; } \\
\text { Volume 2/3: Section } 3 \\
\text { "Definitions" }\end{array}$ & \\
\hline c & Definition of stress intensities & Yes & Vol 2/3 Section 3.5. & $\begin{array}{l}\text { R5 avoids use of the term 'stress intensity' as the term applies to fracture } \\
\text { mechanics applications in other parts of R5. The terms equivalent stress is used } \\
\text { rather than 'stress intensity', and is based upon Mises. }\end{array}$ \\
\hline d & Definition of strain intensities & No & Vol 2/3 Section 3.5. & Equivalent strain (Mises) is used rather than 'strain intensity'. \\
\hline e & $\begin{array}{l}\text { Definition of primary vs secondary } \\
\text { stresses }\end{array}$ & Yes & $\begin{array}{l}\text { Vol 2/3 Section 3.6; } \\
\text { Vol } 1 \text { Section } 5.2\end{array}$ & $\begin{array}{l}\text { ASME NH and RCC-MR are referenced; however, there are no tabulated } \\
\text { example of how to classify as in ASME NH. }\end{array}$ \\
\hline $\mathrm{f}$ & $\begin{array}{l}\text { Definition of membrane, bending, } \\
\text { peak stresses }\end{array}$ & Yes & Vol 2/3 Section 3.6 & \\
\hline g & Procedure for stress linearization & No & $\begin{array}{l}\text { Vol 2/3 Section 3.6, Appendix } \\
\text { A2 }\end{array}$ & Reference made to PVRC project (Hollinger \& Hechmer). \\
\hline
\end{tabular}


Table 9.2.2.2-2 R5: Design Loads

\begin{tabular}{|c|c|c|c|c|}
\hline ID\# & Definition / Criterion & Covered? & Location & Comment \\
\hline $\mathrm{h}$ & Differentiated load catagories: & & & \\
\hline & design loads & No & & Code is for 'assessment', design loads not addressed. \\
\hline & normal service loads & No & & \\
\hline & frequent abnormal loads & No & & \\
\hline & infrequent abnormal loads & No & $\begin{array}{l}\text { Volume } 1 \text { Section } 2 \& \\
\text { Section } 5.1\end{array}$ & $\begin{array}{l}\text { Scope is limited to loading where } 80 \% \text { of a section ligament is } \\
\text { within elastic shakedown. }\end{array}$ \\
\hline & limiting fault loads & No & $\begin{array}{l}\text { Volume } 1 \text { Section } 2 \& \\
\text { Section } 5.1\end{array}$ & $\begin{array}{l}\text { Interaction with severe dynamic loading which may occur during } \\
\text { seismic events is not considered. }\end{array}$ \\
\hline & test loads & No & & Code is for 'assessment'. \\
\hline $\mathrm{i}$ & $\begin{array}{r}\text { Flow chart for design by analysis } \\
\text { or rule/procedure }\end{array}$ & Yes & Vol 2/3 Section 4 & Figure 4.1(a) - Figure 4.1(d) \\
\hline $\mathrm{j}$ & $\begin{array}{r}\text { Yield function requirements (e.g. } \\
\text { Tresca vs Mises) }\end{array}$ & Yes & Vol 2/3 Section 3.5. & Mises \\
\hline
\end{tabular}


Table 9.2.2.3-1 R5: Failure Mechanisms

\begin{tabular}{|c|c|c|c|c|}
\hline ID\# & Definition / Criterion & Covered? & Location & Comment \\
\hline a & $\begin{array}{l}\text { Limit load collapse, single load } \\
\text { application }\end{array}$ & Yes & Vol 2/3 Section 1, 4, \& 5.2; R66 & Sy: min $0.2 \%$ proof stress. \\
\hline $\mathrm{b}$ & $\begin{array}{l}\text { Excessive deformation limiting } \\
\text { functionality, under a single load } \\
\text { application }\end{array}$ & Yes/No & Vol 2/3 Section 6.3 & Safety factors on Sy are used; indirectly address this concern. \\
\hline c & $\begin{array}{l}\text { Structural instability or buckling, } \\
\text { under a single load application }\end{array}$ & No & Vol 1 Section 3 & $\begin{array}{l}\text { Buckling is deliberately not considered; predominately pressure } \\
\text { vessels under tensile loading, similar case for internals components of } \\
\text { AGR. }\end{array}$ \\
\hline d & $\begin{array}{l}\text { Progressive collapse by } \\
\text { ratcheting under cyclic load }\end{array}$ & Yes & $\begin{array}{l}\text { Vol 2/3 Section } 1,4,5 \text {; data } \\
\text { requirements 5.1, 5.2, 5.3, 5.6, 5.7, } \\
\text { 5.8, 5.9, 5.10; Appendix A1; R66. }\end{array}$ & $\begin{array}{l}\text { Elastic and physical constants, monotonic tensile data, cyclic stress- } \\
\text { strain data, } \mathrm{S}_{\mathrm{R}} \text { curves (rupture), isochronous stress-strain data } \\
\text { (deformation/strain), stress relaxation data (in cyclically conditioned } \\
\text { state). } \\
\text { (Structural behavior limited to shakedown or elastic behavior; limited } \\
\text { constrained plasticity is permitted.) }\end{array}$ \\
\hline e & Nonductile fracture & Yes & $\begin{array}{l}\text { Vol 2/3 Section 1, 5.7, 6.5, } \\
\text { Appendix A1.7; R66. }\end{array}$ & $\begin{array}{l}S_{R} \text { curves (creep rupture) in R66; rupture reference stress varies } \\
\text { according to extent of creep ductile vs. creep brittle. }\end{array}$ \\
\hline $\mathrm{f}$ & $\begin{array}{l}\text { Fatigue failure (nucleation on } \\
\text { order of } 5 \mathrm{~mm} \text { ) }\end{array}$ & Yes & $\begin{array}{l}\text { Vol 2/3 Section 1, 4, 5.5, Appendix } \\
\text { A1.5; R66. }\end{array}$ & $\begin{array}{l}\text { Continuous cycling fatigue data in R66; other resources noted: } \\
\text { ASME NH, RCC-MR, NRIM. }\end{array}$ \\
\hline g & $\begin{array}{l}\text { Collapse or breach of pressure } \\
\text { boundary due to corrosion, mass } \\
\text { transfer phenomenon, etc. }\end{array}$ & No & Vol 1 Section 3 & Corrosion is deliberately not considered; may be considered in future. \\
\hline h & $\begin{array}{l}\text { Excessive deformation leading to } \\
\text { loss of functionality, due to creep } \\
\text { under steady load }\end{array}$ & Yes/No & Vol 2/3 Section 3.7 & $\begin{array}{l}\mathrm{S}_{\mathrm{R}} \text { governed by lower of rupture and strain limit (1\% ferritics, } 2 \% \\
\text { austenitics). }\end{array}$ \\
\hline
\end{tabular}


Table 9.2.2.3-2 R5: Failure Mechanisms

\begin{tabular}{|c|c|c|c|c|}
\hline ID\# & Definition / Criterion & Covered? & Location & Comment \\
\hline $\mathrm{i}$ & Creep rupture & Yes & $\begin{array}{l}\text { Vol 2/3 Section 1, 4, 5.8, Appenidx } \\
\text { A1.8; R66. }\end{array}$ & $\begin{array}{l}\mathrm{S}_{\mathrm{R}} \text { curves (creep rupture) in R66; other resources noted: FR Data and } \\
\text { Conventions Manual, BS PD6525, ECCC Data Sheets, NRIM. }\end{array}$ \\
\hline $\mathrm{j}$ & Creep buckling & No & & $\begin{array}{l}\text { Buckling is deliberately not considered; predominately pressure } \\
\text { vessels under tensile loading, similar case for internals components of } \\
\text { AGR. }\end{array}$ \\
\hline $\mathrm{k}$ & Enhanced creep & Yes & Same as 'd' above. & Same as 'd' above. \\
\hline 1 & Creep-Fatigue & Yes & $\begin{array}{l}\text { Vol 2/3 Section 1, 4, 5.5, 5.6, 5.11, } \\
\text { Appendix A1.11; R66 Section } 7 .\end{array}$ & $\begin{array}{l}\text { Continuous cycling fatigue data in R66; other resources noted: ASME } \\
\text { NH, RCC-MR, NRIM. } \\
\text { Creep ductility data as function of strain rate in R66. }\end{array}$ \\
\hline $\mathrm{m}$ & Accelerated creep rupture & Yes & Same as 'd' above. & Same as 'd' above. \\
\hline $\mathrm{n}$ & $\begin{array}{l}\text { Softening enhanced cyclic creep } \\
\text { rupture }\end{array}$ & No & $\begin{array}{l}\text { Vol 2/3 Section 5.10, 5.11, } \\
\text { Appendix A1.10, Appendix A11. }\end{array}$ & $\begin{array}{l}\text { R5 recognizes that stress and strain (deformation) behavior may differ } \\
\text { from monotonic vs. cyclic loading, e.g. Ks accounts for cyclic } \\
\text { hardening/softening of Sy. No modifications are made to } S_{R} \text { curves to } \\
\text { account for this, if the mechanism exists. However, consideration of } \\
\text { stress relaxation rate in cyclic state impacts rate of 'damage' } \\
\text { accumulation in ductility exhaustion C-F failure calculation. }\end{array}$ \\
\hline o & $\begin{array}{l}\text { Softening enhanced cyclic creep } \\
\text { deformation }\end{array}$ & Yes & $\begin{array}{l}\text { Vol 2/3 Section 5.3, 5.4, 5.10, } \\
\text { Appendix A.10, Appendix A11. }\end{array}$ & $\begin{array}{l}\text { As in 'm' above, modification of stress-strain behavior as a function of } \\
\text { cyclically stable state is included; specifically, modification of Sy for } \\
\text { shakedown assessment, and modification of stress-relaxation for } \\
\text { assessment of strain increment during C-F assessment. Permissible } \\
\text { deformation (limits) is independent of cyclic softening/hardening. }\end{array}$ \\
\hline $\mathrm{p}$ & Irradiation effects & No & & $\begin{array}{l}\text { R66 may include irradiation effects, unkown as R66 unavailable to } \\
\text { public. }\end{array}$ \\
\hline
\end{tabular}


Table 9.2.2.3-3 R5: Failure Mechanisms

\begin{tabular}{|c|l|c|l|l|}
\hline ID\# & \multicolumn{1}{|c|}{ Definition / Criterion } & Covered? & \multicolumn{1}{|c|}{ Location } & \multicolumn{1}{|c|}{ Comment } \\
\hline q & weldments (strength, strain) & Yes & $\begin{array}{l}\text { Appedix A5 } \\
\text { Volume 6. }\end{array}$ & $\begin{array}{l}\text { Fatigue Strength Reduction Factors for various weldment types } \\
\text { and materials (Table A4.1-A4.3 \& Figure A4.1). } \\
\text { Use of different S } \text { R data for parent,weld, and HAZ spatially } \\
\text { throughout section- coupled with analysis in determination of } \\
\text { reference stress and reference temperature. * Note, FSRFs are } \\
\text { dependent upon the analysis procedure; substitution and direct } \\
\text { comparision of other FSRFs (e.g. ASME NH) are not possible. } \\
\text { Dissimilar welds are treated in detail in Vol 6. }\end{array}$ \\
\hline $\mathrm{r}$ & $\begin{array}{l}\text { Multiaxial effects on creep, fatigue, } \\
\text { C-F }\end{array}$ & Yes & $\begin{array}{l}\text { Vol 2/3 Section 1, 4, 5.5, 5.6, 5.11, } \\
\text { Appendix A1.11; R66 Section 7. }\end{array}$ & $\begin{array}{l}\text { Similar to 'k' above; also, effects of strain rate and stress state on } \\
\text { creep ductility are taken into account. }\end{array}$ \\
\hline $\mathrm{s}$ & Local vs. distributed damage & Yes & $\begin{array}{l}\text { Vol 2/3 Section 1, 4, 5.7, Appendix } \\
\text { A1.7; R66. }\end{array}$ & $\begin{array}{l}\text { Creep ductile if ratio fracture strain to Monkman-Grant strain >= } \\
\text { permissible 'usage factor'.) }\end{array}$ \\
\hline $\mathrm{t}$ & Thermal aging & Yes/No & Vol 2/3 Appendix A1; R66. & $\begin{array}{l}\text { Thermal aging is noted to possibly affect fatigue endurance; } \\
\text { references provided. Testing of service exposed material is sited } \\
\text { and recommended. Uncertain if R66 contains guidance (unlikely). }\end{array}$ \\
\hline $\mathrm{u}$ & Elastic follow-up & Yes & $\begin{array}{l}\text { Vol 2/3 Section 3.8, 4, 7.3, } \\
\text { Appendix 8. }\end{array}$ & $\begin{array}{l}\text { Various options with varying degrees of conservatism and effort } \\
\text { are available. }\end{array}$ \\
\hline
\end{tabular}


Table 9.2.2.4-1 R5: Design Criteria / Procedures

\begin{tabular}{|l|l|l|l|l|l|l|l|l|}
\hline & & & & & \\
\end{tabular}


Table 9.2.2.4-2 R5: Design Criteria / Procedures

\begin{tabular}{|c|c|c|c|c|c|c|c|c|c|}
\hline 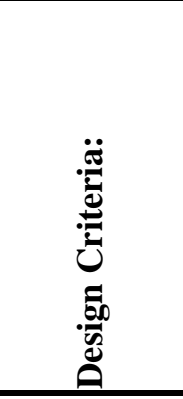 & 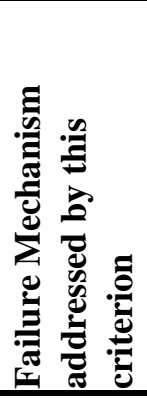 & 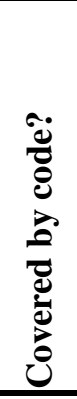 & 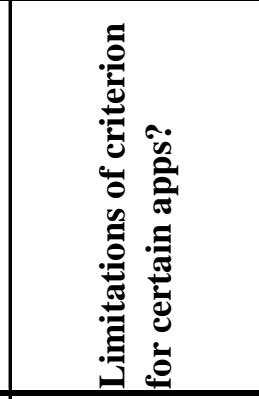 & 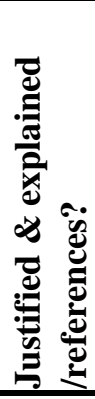 & 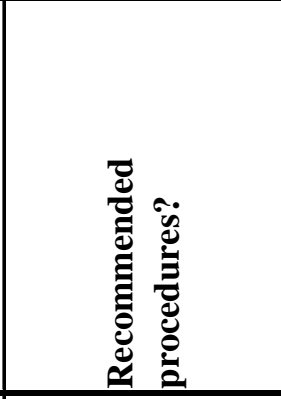 & 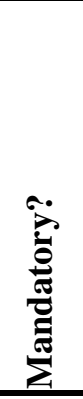 & 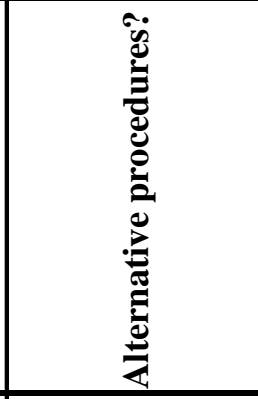 & 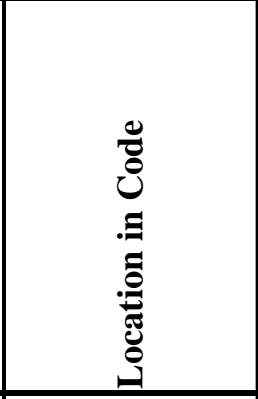 & 葛 \\
\hline $\begin{array}{c}\text { iv. } \\
\text { Ratcheting }\end{array}$ & d & Yes. & $\begin{array}{l}\text { Simplified analysis } \\
\text { does not permit } \\
\text { loading in the } \\
\text { 'ratcheting' regime; } \\
\text { inelastic analysis } \\
\text { required in such } \\
\text { cases. }\end{array}$ & Yes. & $\begin{array}{l}\text { Global shakedown } \\
\text { (all points in structure } \\
\text { shakedown). } \\
\text { Reference stress } \\
\text { approach. }\end{array}$ & Yes. & $\begin{array}{l}\text { Any procedure that } \\
\text { satisfies shakedown } \\
\text { criteria. }\end{array}$ & $\begin{array}{l}\text { Vol 2/3 Section } \\
\text { 6.6, Appendix A6 }\end{array}$ & $\begin{array}{l}\text { Definition of full inelastic } \\
\text { analysis is not provided. }\end{array}$ \\
\hline v. Fracture & e & Yes. & & Yes. & $\begin{array}{l}\text { R5 and its low } \\
\text { temperature } \\
\text { counterpart R6 were } \\
\text { developed with an } \\
\text { emphasis on } \\
\text { procedures to assess } \\
\text { structures with } \\
\text { flaws/defects. }\end{array}$ & Yes. & & $\begin{array}{l}\text { Vol 4/5, and R6 for } \\
\text { low temperature. }\end{array}$ & $\begin{array}{l}\text { 304SS, 316SS, } 800 \mathrm{H} \text { must be } \\
\text { reconsidered if fabrication } \\
\text { alters fracture mode to brittle. }\end{array}$ \\
\hline vi. Fatigue & $\mathrm{f}$ & Yes. & $\begin{array}{l}\text { Severe differences } \\
\text { in strain ranges } \\
\text { throughout service } \\
\text { may require } \\
\text { specific crack } \\
\text { growth analysis } \\
\text { such as in Vol 4/5. }\end{array}$ & No. & Miner's rule. & Yes. & $\begin{array}{l}\text { Thin sections: } \\
\text { procedure for } \\
\text { partitioning crack } \\
\text { nulcation and } \\
\text { propagation to } \\
\text { appropriate size. } \\
\text { Sequence effects - } \\
\text { see Vol 4/5. }\end{array}$ & $\begin{array}{l}\text { Vol 2/3 Section } \\
\text { 8.2, Appendix A10. }\end{array}$ & $\begin{array}{l}\text { Fatigue addressed in creep- } \\
\text { fatigue criteria; if insignificant } \\
\text { creep. Criteria covered under } \\
\text { C-F within this table. }\end{array}$ \\
\hline
\end{tabular}


Table 9.2.2.4-3 R5: Design Criteria / Procedures

\begin{tabular}{|c|c|c|c|c|c|c|c|c|c|}
\hline 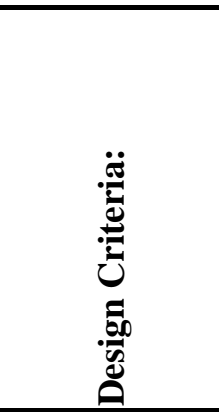 & 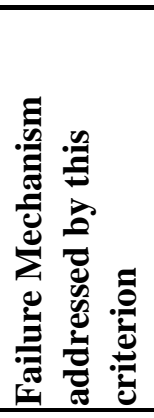 & 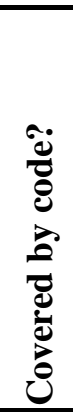 & 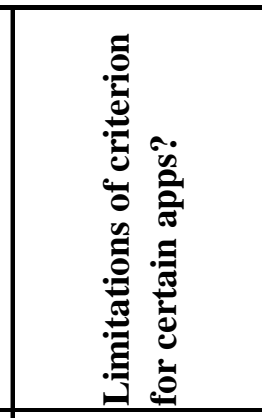 & 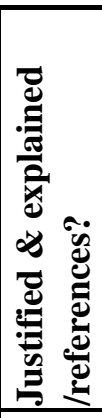 & 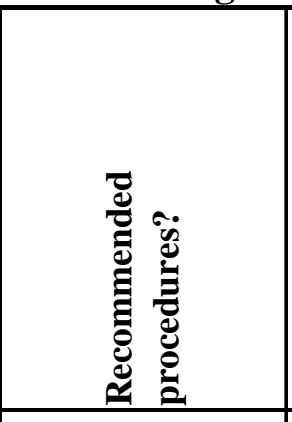 & 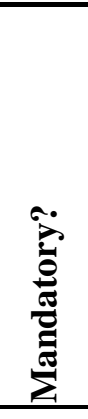 & 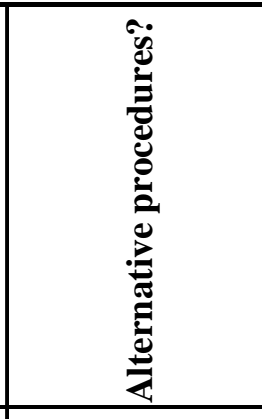 & 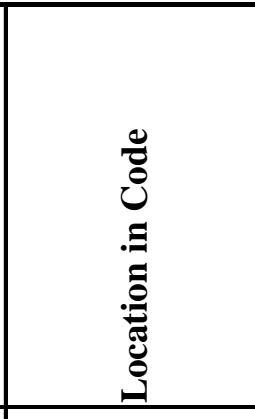 & 晜 \\
\hline vii. Corrosion & g & No. & & & & & & & \\
\hline $\begin{array}{l}\text { viii. Excessive } \\
\text { creep } \\
\text { deformation }\end{array}$ & $\mathrm{h}$ & Yes. & $\begin{array}{l}\text { Creep ductile and } \\
\text { brittle materials } \\
\text { addressed by } \\
\text { means of } \\
\text { modifying } \\
\text { reference stress } \\
\text { according to extent } \\
\text { of creep ductility. }\end{array}$ & Yes. & \begin{tabular}{|l|} 
Creep usage factor \\
rule applied to \\
shakedown reference \\
stress (not identical to \\
'core stress'); \\
permissible \\
deformation is $1 \%$ \\
(ferritics) or $2 \%$ \\
(austenitics).
\end{tabular} & Yes. & $\begin{array}{l}\text { Options to evaluate } \\
\text { reference stress } \\
\text { (primary stress): } \\
\text { 1) Stress } \\
\text { linearization } \\
\text { approach } \\
\text { 2) Elastic finite } \\
\text { element approach } \\
\text { 3) Various } \\
\text { reference stress } \\
\text { approaches: } \\
\text { compendia of limit } \\
\text { load solutions, } \\
\text { inversion of design } \\
\text { Codes, limit } \\
\text { analysis, solutions } \\
\text { published in } \\
\text { literature, finite } \\
\text { element analysis. }\end{array}$ & $\begin{array}{l}\text { Vol 2/3 Section } \\
6.5, \text { Appendix A5. }\end{array}$ & $\begin{array}{l}\text { Strain is limited to } 1 \%, 2 \% \text { \& } \\
5 \% \text {, AND stress intensity is } \\
\text { dictated by limiting strain to } 1 \%\end{array}$ \\
\hline $\begin{array}{c}\text { ix. Creep } \\
\text { buckling }\end{array}$ & h,b & No. & & & & & & & \\
\hline
\end{tabular}


Table 9.2.2.4-4 R5: Design Criteria / Procedures

\begin{tabular}{|c|c|c|c|c|c|c|c|c|c|}
\hline 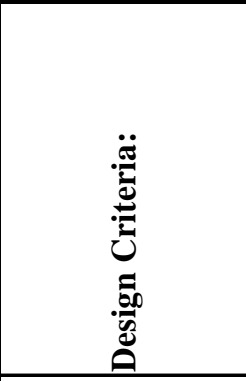 & 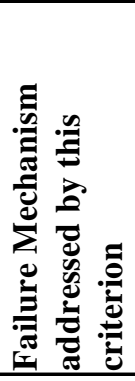 & 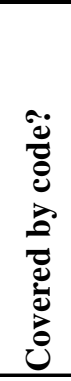 & 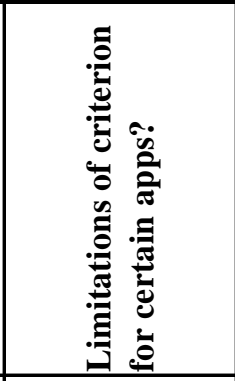 & 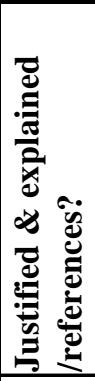 & 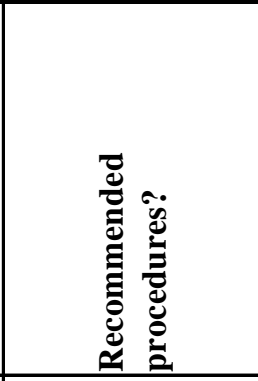 & 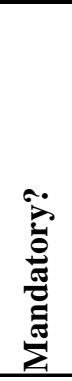 & 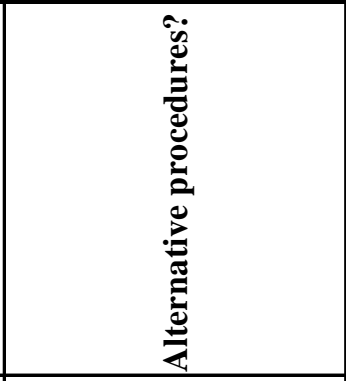 & 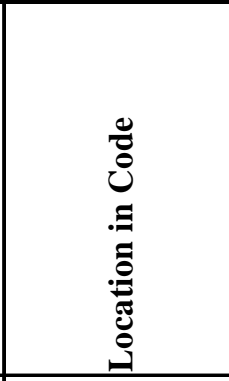 & 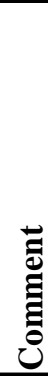 \\
\hline x. Creep rupture & b,h,i & Yes. & $\begin{array}{l}\text { Creep ductile and } \\
\text { brittle materials } \\
\text { addressed by } \\
\text { means of } \\
\text { modifying } \\
\text { reference stress } \\
\text { according to extent } \\
\text { of creep ductility. }\end{array}$ & Yes. & $\begin{array}{l}\text { Creep usage factor. } \\
\text { Reference stress is } \\
\text { approach of choice. }\end{array}$ & Yes. & $\begin{array}{l}\text { Options to evaluate reference } \\
\text { stress (primary stress): } \\
\text { 1) Stress linearization } \\
\text { approach } \\
\text { 2) Elastic finite element } \\
\text { approach } \\
\text { 3) Various reference stress } \\
\text { approaches: compendia of } \\
\text { limit load solutions, } \\
\text { inversion of design Codes, } \\
\text { limit analysis, solutions } \\
\text { published in literature, finite } \\
\text { element analysis. }\end{array}$ & $\begin{array}{l}\text { Vol 2/3 Section } \\
\text { 6.5, Appendix A5. }\end{array}$ & \\
\hline $\begin{array}{c}\text { xi. } \quad \text { Enhanced } \\
\text { Creep Deformation }\end{array}$ & $\mathrm{j}$ & Yes. & $\begin{array}{l}\text { Simplified analysis } \\
\text { does not permit } \\
\text { loading in the } \\
\text { 'ratcheting' regime; } \\
\text { inelastic analysis } \\
\text { required in such } \\
\text { cases. }\end{array}$ & Yes. & $\begin{array}{l}\text { Creep usage factor } \\
\text { rule applied to } \\
\text { shakedown reference } \\
\text { stress (i.e. core } \\
\text { stress); permissible } \\
\text { deformation is } 1 \% \\
\text { (ferritics) or } 2 \% \\
\text { (austenitics). }\end{array}$ & Yes. & $\begin{array}{l}\text { Solutions for geometry and } \\
\text { load specific cases; inelastic } \\
\text { analysis. }\end{array}$ & $\begin{array}{l}\text { Vol 2/3 Section } \\
\text { 7.5, Appendix A9. }\end{array}$ & \\
\hline
\end{tabular}


Table 9.2.2.4-5 R5: Design Criteria / Procedures

\begin{tabular}{|c|c|c|c|c|c|c|c|c|c|}
\hline 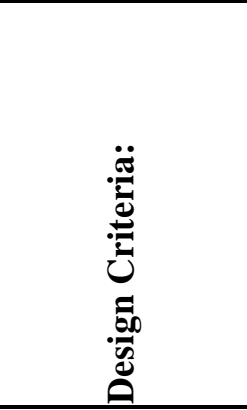 & 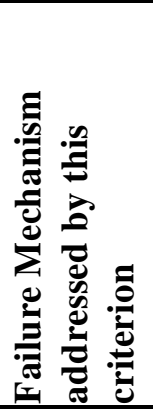 & 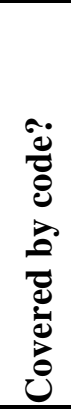 & 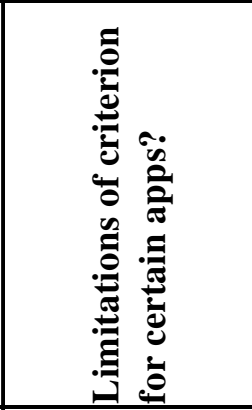 & 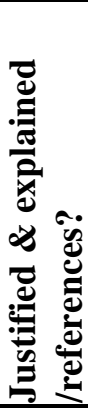 & 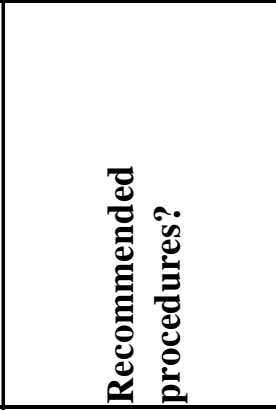 & 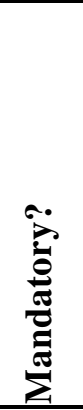 & 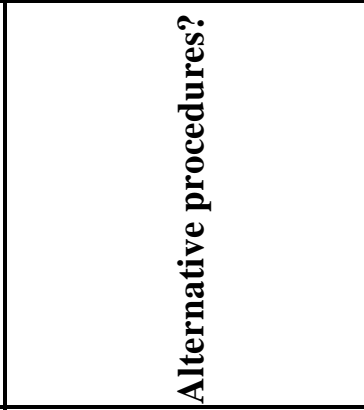 & 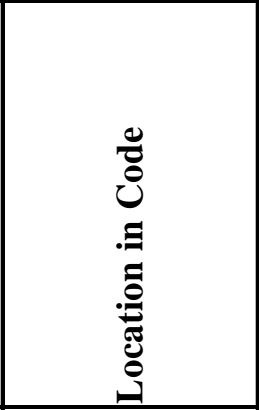 & 葛 \\
\hline $\begin{array}{l}\text { xii. } \quad \text { C-F } \\
\text { Interaction }\end{array}$ & $\mathrm{k}$ & Yes. & $\begin{array}{l}\text { Linear damage rule } \\
\text { limitations. }\end{array}$ & Yes. & Linear damage rule. & Yes. & $\begin{array}{l}\text { Crack growth procedures in } \\
\text { Vol } 4 / 5 .\end{array}$ & $\begin{array}{l}\text { Vol 2/3 Section 7, } \\
\text { 8, Appendix A7, } \\
\text { A8, A10, A11. }\end{array}$ & \\
\hline $\begin{array}{l}\text { xiii. Accelerated } \\
\text { Cyclic Creep } \\
\text { Rupture }\end{array}$ & l & Yes. & $\begin{array}{l}\text { Simplified analysis } \\
\text { does not permit } \\
\text { loading in the } \\
\text { 'ratcheting' regime; } \\
\text { inelastic analysis } \\
\text { required in such } \\
\text { cases. }\end{array}$ & Yes. & $\begin{array}{l}\text { Creep rupture usage } \\
\text { factor rule applied to } \\
\text { shakedown reference } \\
\text { stress (i.e. core } \\
\text { stress). }\end{array}$ & Yes. & $\begin{array}{l}\text { Solutions for geometry and } \\
\text { load specific cases; inelastic } \\
\text { analysis. }\end{array}$ & $\begin{array}{l}\text { Vol 2/3 Section } \\
\text { 7.5, Appendix A9. }\end{array}$ & \\
\hline $\begin{array}{l}\text { xiv. Softening } \\
\text { Enhanced Cyc. } \\
\text { Creep } \\
\text { Deformation }\end{array}$ & $\mathrm{m}$ & No. & \multicolumn{6}{|c|}{$\begin{array}{l}\text { While cyclically stable yield strength is utilized to arrive at more appropriate reference stress, no modification } \\
\text { is made directly to } S_{R} \text { to account for increased cyclic creep deformation rates. }\end{array}$} & \\
\hline $\begin{array}{l}\text { xv. Softening } \\
\text { Enhanced Cyc. } \\
\text { Creep Rupture }\end{array}$ & $\mathrm{n}$ & No. & \multicolumn{6}{|c|}{$S_{R}$ is not modified to account for reduced rupture times due to cyclic creep. } & \\
\hline
\end{tabular}


Table 9.2.2.4-6 R5: Design Criteria / Procedures

\begin{tabular}{|c|c|c|c|c|c|c|c|c|c|}
\hline 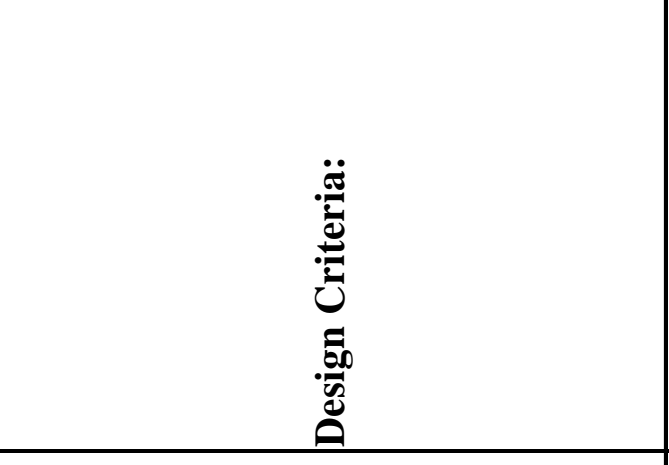 & 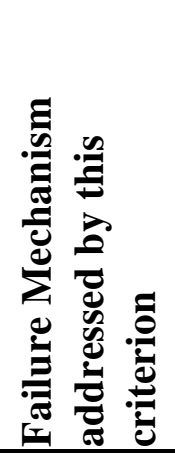 & 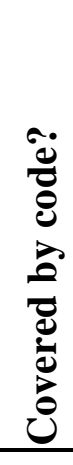 & 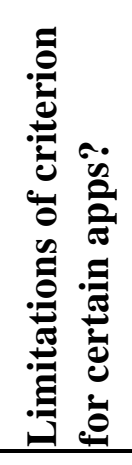 & 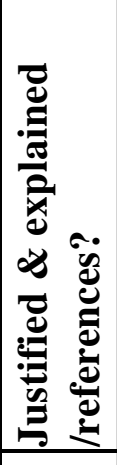 & 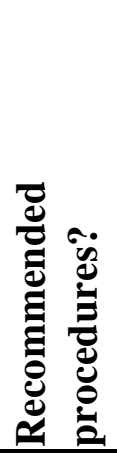 & 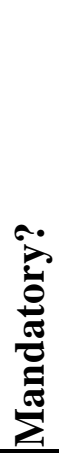 & 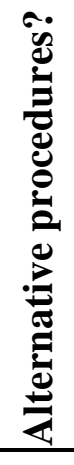 & 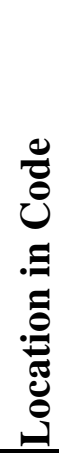 & 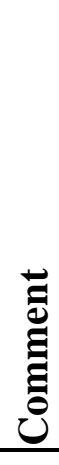 \\
\hline xvi. Irradiation induced swelling & 0 & No. & & & & & & & \\
\hline xvii. Irradiation induced creep & 0 & No. & & & & & & & \\
\hline $\begin{array}{l}\text { xviii. Plastic Flow localization due to } \\
\text { irradiation (dislocation tunneling) }\end{array}$ & 0 & No. & & & & & & & \\
\hline $\begin{array}{c}\text { xix. Ductility exhaustion due to irradiation } \\
\text { exposure }\end{array}$ & 0 & No. & & & & & & & \\
\hline $\begin{array}{l}\text { xx. Irradiation induced effects on fatigue, } \\
\text { and on C-F }\end{array}$ & 0 & No. & & & & & & & \\
\hline
\end{tabular}


Table 9.2.2.4-7 R5: Design Criteria / Procedures

\begin{tabular}{|c|c|c|c|c|c|c|c|c|c|}
\hline 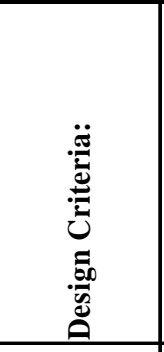 & 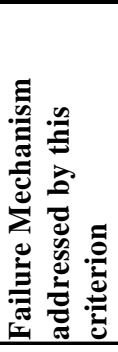 & 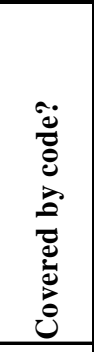 & 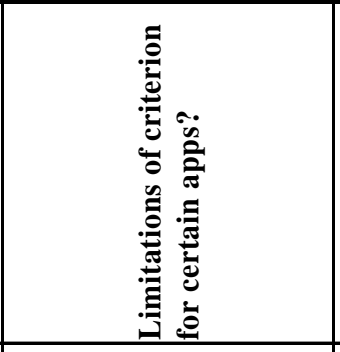 & 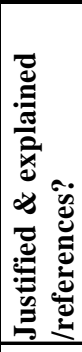 & 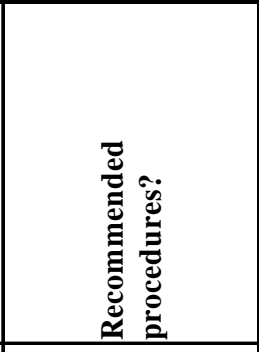 & 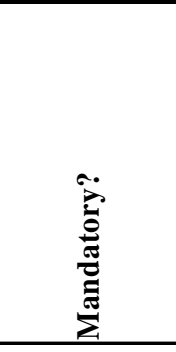 & 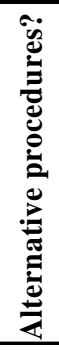 & 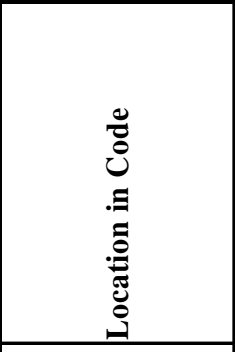 & 莽 \\
\hline $\begin{array}{c}\text { xxi. Local } \\
\text { vs. non-local } \\
\text { damage }\end{array}$ & $\mathrm{p}$ & Yes. & \begin{tabular}{|l|} 
Stress concentrations greater \\
than 4 are considered \\
sufficiently sharp to be required \\
to be treated as cracks; must use \\
Vol $4 / 5$ in such cases.
\end{tabular} & Yes. & $\begin{array}{l}\text { Reference stress } \\
\text { approach modified to } \\
\text { account for extent of } \\
\text { creep ductility. }\end{array}$ & Yes. & No. & $\begin{array}{l}\text { Vol 2/3 Section 6.5, } \\
\text { Appendix A5. }\end{array}$ & \\
\hline $\begin{array}{c}\text { xxii. } \\
\text { Thermal } \\
\text { aging effects } \\
\text { on creep, } \\
\text { fatigue, limit } \\
\text { load }\end{array}$ & $\mathrm{q}$ & Yes/No. & \begin{tabular}{|l|} 
Aging effects on creep are \\
deemed inherent to SR data; \\
Fatigue strength reduction \\
factors are noted for thermal \\
aging effects. \\
R66 may have modifications on \\
yield or ultimate strength \\
(unavailable).
\end{tabular} & Yes. & & No. & & Vol 2/3 Appendix A1. & \\
\hline $\begin{array}{c}\text { xxiii. } \\
\text { Elastic } \\
\text { Follow-up }\end{array}$ & $\mathrm{r}$ & Yes. & \begin{tabular}{|l|} 
Limitations apply to some \\
options for evaluating elastic \\
follow-up, such as loading type \\
and levels, and spatial \\
temperature distribution.
\end{tabular} & Yes. & $\begin{array}{l}\text { Three options available, } \\
\text { with varying levels of } \\
\text { effort inversely } \\
\text { proportional to extent of } \\
\text { conservatism. }\end{array}$ & $\begin{array}{l}\text { Yes, for } \\
\text { determination of } \\
\text { strain range for } \\
\text { C-F analysis. }\end{array}$ & & $\begin{array}{l}\text { Vol 2/3 Section 7.3, } \\
\text { Appendix A8. }\end{array}$ & \\
\hline $\begin{array}{c}\text { i. Multi } \\
\text { axial effects } \\
\text { on creep, } \\
\text { fatigue and C- } \\
\text { F }\end{array}$ & $\mathrm{s}$ & Yes. & Need to fill in... & & & & & & \\
\hline
\end{tabular}




\subsubsection{Monju}

The 'Monju' Code is shorthand for the Japanese Structural Design Code for Class I Components of Prototype Fast Breeder Reactor for Elevated Temperature. It is the only example of Japanese design philosophy in this field available in English, on the timescale of this project.

While it does not specifically restrict its usage it is clear that its context is dictated by the application called out in the title. In this respect it focuses on the same aspects of design which governed the development of the Nuclear Codes leading up to Subdivision NH. The similarities of Monju and NH outweigh their differences by a considerable margin. Therefore, in presenting this summary of Monju only those features which differ in substance from $\mathrm{NH}$ will be discussed explicitly.

The Monju Code is vintage 2002 and has since been superseded by new guidelines. Unfortunately these are not available at the time of writing, but a summary of the events of the past decade of progress in Japan was published posthumously by Professor Yasuhide Asada, in the ASME Journal of Pressure Vessel Technology, in 2006, in the form of a 2 part paper, entitled, "Japanese Activities Concerning Nuclear Codes and Standards - Parts I and II”. While these publications do not discuss detailed design procedures they provide a very useful insight into the background of code development in Japan, some of which gives thought to ways in which ASME codes might follow in their future development.

A feature of nuclear design code development in Japan is that detailed technical specifications were defined in a MITI issued Notification \#501 and, in the past, only procedures covered in this document have been accepted by the Japanese regulatory authorities. It is believed there is some parallel here with the situation in the USA with respect to the USNRC. According to Asada, this process was recognized as being slow to respond to new technical developments, and has been superseded by a new, consensual process whose origins lay in the Japanese society of Mechanical Engineers. No details are given of this consensual procedure. It is possible that the change has brought Japanese practice closer to the approach used since its inception by ASME but there could be something to learn from it in terms of achieving rapid response to technical change, which does not appear to be one of the more outstanding features of the ASME approach to code development.

\subsubsection{Monju - High Temperature}

From a qualitative point of view, i.e. in format, loading classifications, design criteria, stress classification, designation of design allowables, etc., the Japanese methodology, to the extent that it can be assumed to be represented by the Monju code, does not differ substantially from $\mathrm{NH}$. 
Several technical considerations differ in detail from their ASME counterparts. Bearing in mind that the Japanese codes are aimed at nuclear power plant construction in general, and not specifically at high temperature applications alone, not all the differences are directly relevant to possible future changes in $\mathrm{NH}$.

Changes which have been identified explicitly are,

- A revised $\mathrm{K}_{1 \mathrm{R}}$ curve for fracture toughness (not directly relevant to ETD)

- Identification of a new failure mechanism related to seismic loading, referred to as "ratcheting fatigue". No details are given but this appears to be a LCF mechanism accelerated by progressive cyclic deformation and is found to occur mostly in piping. The mechanism is short term and not directly creep related but can be superimposed on standard operation, so it is potentially a high temperature issue as well as a low temperature one.

- Seismic disturbance is a major issue in Japan. As a result, design allowables for elevated temperature operation include special rules for dealing with severe short term, usually cyclic, loading at elevated temperature. The $\mathrm{NH}$ equivalent to this feature is not clear.

- Like the ASME Code, the Japanese procedures accept both elastic and inelastic design options. As part of the elastic approach, the $\mathrm{K}_{\mathrm{e}}$ factor, used to compensate for local yielding prior to checking shakedown, has been radically modified from the ASME version. The Japanese version is considerably less conservative.

- Also as part of the elastic route, the Japanese rules address the question of elastic followup in detail. An explicit methodology is supplied to evaluate followup in piping as part of the process of identifying primary and secondary stress components. This methodology calls on the use of the "elastic compensation" method, but this detail becomes less important with time as inelastic analysis, even of the simplified variety, is growing progressively more routine.

- Simplified elastic followup is also incorporated in a new procedure for the evaluation of creep/fatigue interaction. This procedure includes an integrated approach using special specimens which incorporate followup in the material test, thereby dispensing with the need for a separate creep/fatigue damage evaluation altogether. It is not clear from available documents whether this approach has been implemented so far.

- Asada refers to an alternative "strength evaluation method without stress classification” based on limit load analysis. The concepts of primary (load bearing) and secondary (self-equilibrating) stress states are retained, so the expression "without stress classification ...." presumably refers to the standardized procedure first instituted by ASME and since adopted by most equivalent codes, involving linearization of stresses on sections and factorization into P, Q and F stresses. In the Japanese approach, primary load integrity is assured using the Reference Stress concept. Cyclic stability, or avoidance of ratcheting is assured by a modification of the existing ASME " $3 \mathrm{~S}_{\mathrm{M}}$ " limit on primary + secondary, using instead, the full local cyclic stress 
range, but defining a "cyclic yield area" (CYA), which is required to extend less than $10 \%$ through the wall thickness. This procedure eliminates the distinction, retained in the ASME Code, between "Q" and "F" stresses. It bears some similarity to the R5 practice. The approach is only outlined for time independent applications, but the extension to high temperatures is clear.

\subsubsection{Monju - Design Loads}

The design load classification set out in ASME Section III is used virtually unchanged in the Japanese procedures, although, for obvious reasons, more explicit guidelines are provided for dealing with the effects of seismic loading.

Table 9.2.3.2-1 and 9.2.3.2-2 summarize information relevant to 'Design Loads' for an Ideal ETD Code, including the Definition / Criterion, whether the Definition is 'Covered', the location in the Code where the Definition is addressed, and relevant comments. Of particular interest are the following:

- Significant guidance is given for development of a load histogram and cycle definitions where the cycle of interest is the steady cyclic state; while noted in general, the transient effects from one cycle to another - or early behavior in route to establishment of the cyclic state - are assumed to be negligible.

- The fundamental use and understanding of primary and secondary stresses, membrane, local, bending, and peak stresses are utilized as one option. The wording of the Monju document appears to allow greater flexibility on the part of the designer in determining these classifications however. The difficulty, if not impossibility of carrying out such a classification in the case of complex 3D geometries is recognized. As a result, design criteria and procedures permit use of an alternative reference stress approach and allied methods for assessing ratcheting which do not require stress linearization or explicit categorization, although the distinction between primary and secondary stresses is conserved.

- Differentiation of load categories follows ASME Code practice. However, two important aspects are:

o structural behavior is limited to elastic, shakedown, or plasticity; loading into the ratcheting regime is recognized as potentially unavoidable in the event of an earthquake.

o Greater recognition than is found in Section III NB or NH is accorded to the use of inelastic analysis, whether by simplified methods such as "elastic compensation" or full and detailed FEA.

- Thermal loading is given a much more detailed treatment than is provided in Section III, NB or NH. Thermal striping and stratification are both called out as problems to be considered specifically in design of piping systems. 


\subsubsection{Monju - Failure Mechanisms}

The Japanese procedures address all the same mechanical and environmentally accelerated failure mechanisms considered in Section III, NB and NH.

In addition it names two further mechanisms referred to as "ratcheting fatigue" and "high cycle thermal fatigue”.

- Ratcheting fatigue is specific to resistance to seismic loading and appears to be focused on piping, although there is no specific exclusion of other components which could be made vulnerable to failure by such a mechanism.

- High cycle thermal fatigue involves very high numbers of cycles, possibly significantly higher than is currently considered by the ASME Code $\left(10^{8}\right)$. This mechanism is discussed in the context of thermal loads including thermal striping and stratification which may be covered in Section III, but not with such a high profile as s given to them by the Japanese procedures.

Tables 9.2.3.3-1 thru 9.2.3.3-3 summarize the extent by which failure mechanisms of an Ideal ETD Code are addressed by the Monju procedure. The information is summarized in the same manner as Tables 9.2.3.2-1 and 9.2.3.2-2. Three possibilities exist for response to the column labeled 'Covered?', "Yes”, "No", and "Yes/No", with the latter requiring an explanation. "Yes/No" indicates that the Code does not explicitly address the failure mechanism or does in a limited way or circumstances; but the Code does address the mechanism indirectly to some extent. For example, 'Excessive deformation limiting functionality, under a single load application’ is indirectly covered by safety factors on Sy for limit loads.

\subsubsection{Monju - Design Criteria / Procedures}

Tables 9.2.3.4-1 through 9.2.3.4-7 summarize the various design criteria of an Ideal ETD Code and how the Japanese procedures address them or not. The first column indicates the design criteria, and the failure mechanism addressed by the criterion is listed in the second column; whether or not the R5 Procedure covers the design criteria is indicated in the third column. Limitations of the criteria, justification \& explanation / references for the design criteria, and recommended procedures are also listed, including whether or not they are mandatory or not. Alternative procedures are summarized, including the locations in the Code that address the criteria. Finally, comments are included as appropriate.

The Japanese procedures allow both a simplified method based on linear elastic analysis, almost identical to current Section III practice, as well as inelastic analysis based on detailed FEA for all aspects of design analysis. In this respect they differ from current ASME practice in that Section III NH specifically prohibits inelastic analysis for general ETD, permitting inelastic analysis on a limited basis for those elements of operation 
covered in Appendix T, i.e. strain limits and structural analysis as precursor to assessment of creep/fatigue interaction.

Inelastic analysis based on detailed FEA as an option appears to be motivated largely by the difficulty of stretching the original concept of stress linearization as developed by ASME to accommodate the more complex 3D geometries being considered on a routine basis in current design analysis. It also appears to recognize the revolution occurring in engineering design analysis in recent years which, in many cases, has displaced traditional "hand calculations" with an entirely different paradigm, involving CAD generated structural shapes, meshed automatically and analyzed by "solvers" whose origins in fundamental finite element analysis may no longer be known to the analyst. The elastic route is retained to satisfy conservative designers who continue to use traditional methods.

\subsubsection{Monju - Summary}

In summary, the Japanese procedures for nuclear plant design follow those of ASME Sections III and XI (for surveillance) very closely. This is not surprising since the two Codes have developed in an atmosphere of close cooperation between ASME and JSME. Differences do exist however, and some, if not all, are worthy of examination in the process of updating the ASME procedures. Those affecting ETD specifically are,

- Addition of a new material failure mechanism named "ratcheting fatigue”. In the Japanese context this mechanism is restricted to seismic events, but it is also a fundamental problem which deserves consideration wherever incremental plasticity is permitted. This question arises in the current $\mathrm{NH}$ procedures which allow for the computation of ratcheting deformation but do not specifically prohibit operation in the ratcheting regime.

- A trend, rather than a distinct difference between the Japanese and ASME procedures lies in the greater acceptance by the former of de facto analysis practices involving the routine use of inelastic FEA. There has been a radical paradigm shift in this area which has yet to be fully recognized by any code body, but the Japanese appear to be further along the path, in the design field, than any others.

- The option of abandoning the now traditional stress classification procedure, which has been the mainstay of pressure vessel design internationally for decades, is an innovative step being considered by the Japanese. This step has been taken in codes and guidelines directed at Fitness-for-Purpose assessments (R5, API 579), and for low temperature design within the ASME Code (the new version of Section VIII/Div.2), but has so far is not known to have been adopted anywhere else for ETD. This step involves more than simply the use of a more intricate stress analysis tool. It admits the possibility of much more complex geometric shapes, with the need to pass of more responsibility to the analyst for problem formulation. 
The Japanese procedures, even when presenting the same methods contained in the ASME Code, generally allow a higher level of discretion to be exercised by the designer than does parts of the ASME Code, notably Section III, which is significantly more prescriptive than the non-nuclear sections I and VIII (all versions). 
Table 9.2.3.1 Monju: High Temperature

\begin{tabular}{|c|c|c|c|l|}
\hline ID\# & Definition / Criterion & Covered? & Location & Comment \\
\hline a & High temperature limit & Yes & MITI Note \#501 & Details not available \\
\hline b & $\begin{array}{c}\text { Criterion or definition of } \\
\text { temperature limit for } \\
\text { insignificant creep effects }\end{array}$ & Yes & MONJU Guideline 3.4.2.(3) p.15 & $\mathrm{t} / \mathrm{t}_{\mathrm{R}}<0.1$ \\
\hline
\end{tabular}


Table 9.2.3.2-1 Monju: Design Loads

\begin{tabular}{|c|c|c|c|c|}
\hline ID\# & Definition / Criterion & Covered? & Location & Comment \\
\hline a & $\begin{array}{l}\text { Guidelines for load historgram \& } \\
\text { cycle definition }\end{array}$ & No & & \\
\hline $\mathrm{b}$ & Glossary of terms & Y? & MITI Note \#501 & details not available \\
\hline c & Definition of stress intensities & Yes? & $\begin{array}{l}\text { No specific location. } \\
\text { Terminology adopted generally } \\
\text { through document suggests } \\
\text { defined elsewhere, possiblly } \\
501\end{array}$ & $\begin{array}{l}\text { ASME Code definitions and usage adopted without specific reference. Recent } \\
\text { (2006) Asada document refers to new development termed "classification free", } \\
\text { based on Reference Stress concept }\end{array}$ \\
\hline d & Definition of strain intensities & No & & Equivalent strain (Mises) is used rather than 'strain intensity'. \\
\hline e & $\begin{array}{l}\text { Definition of primary vs secondary } \\
\text { stresses }\end{array}$ & Yes & see c) above & see c) above \\
\hline $\mathrm{f}$ & $\begin{array}{l}\text { Definition of membrane, bending, } \\
\text { peak stresses }\end{array}$ & Yes & Table 2.1 & Monju guideline practice follows ASME \\
\hline g & Procedure for stress linearization & No & see c) above & see c) above \\
\hline
\end{tabular}


Table 9.2.3.2-2 Monju: Design Loads

\begin{tabular}{|c|c|c|c|c|}
\hline ID\# & Definition / Criterion & Covered? & Location & Comment \\
\hline $\mathrm{h}$ & Differentiated load catagories: & & & \\
\hline & design loads & yes & 3.2 .2 & Limits for design \\
\hline & normal service loads & yes & 3.2 .3 & Operation and equiv of ASME I thru IV \\
\hline & frequent abnormal loads & see above & 3.2 .3 & see above \\
\hline & infrequent abnormal loads & see above & 3.2 .3 & see above \\
\hline & limiting fault loads & see above & 3.2 .3 & see above \\
\hline & test loads & yes & 3.2.4 & Test allowables \\
\hline $\mathrm{i}$ & $\begin{array}{r}\text { Flow chart for design by analysis } \\
\text { or rule/procedure }\end{array}$ & No & & Implicit acceptance of ASME procedure without citation \\
\hline $\mathrm{j}$ & $\begin{array}{r}\text { Yield function requirements (e.g. } \\
\text { Tresca vs Mises) }\end{array}$ & Yes & none & Implicit acceptance of ASME procedure without citation \\
\hline
\end{tabular}


Table 9.2.3.3-1 Monju: Failure Mechanisms

\begin{tabular}{|c|c|c|c|c|}
\hline ID\# & Definition / Criterion & Covered? & Location & Comment \\
\hline $\mathrm{a}$ & $\begin{array}{l}\text { Limit load collapse, single load } \\
\text { application }\end{array}$ & Yes? & $\begin{array}{l}\text { Implicitly by use of "P" stresses } \\
\text { throughout }\end{array}$ & $\begin{array}{l}\text { No specific reference made to limit load, but primary structural } \\
\text { strength is dealt with implicitly by adoption of primary stress concept }\end{array}$ \\
\hline $\mathrm{b}$ & $\begin{array}{l}\text { Excessive deformation limiting } \\
\text { functionality, under a single load } \\
\text { application }\end{array}$ & No & & \\
\hline C & $\begin{array}{l}\text { Structural instability or buckling, } \\
\text { under a single load application }\end{array}$ & Yes & Section 4.3 & $\begin{array}{l}\text { No analysis offered per se. SF's provided for specified load } \\
\text { conditions. Does not consider creep. Equations provided only for low } \\
\text { temperature buckling of piping and vessels }\end{array}$ \\
\hline $\mathrm{d}$ & $\begin{array}{l}\text { Progressive collapse by } \\
\text { ratcheting under cyclic load }\end{array}$ & Yes & Section 3.4.(1) b) & $\begin{array}{l}\text { Uses reduced or simplified version of Bree Diagram with } \mathrm{NH} \\
\text { parameter "Z" in equation form only. }\end{array}$ \\
\hline $\mathrm{e}$ & Nonductile fracture & No & & \\
\hline $\mathrm{f}$ & $\begin{array}{l}\text { Fatigue failure (nucleation on } \\
\text { order of } 5 \mathrm{~mm} \text { ) }\end{array}$ & Yes & $\begin{array}{l}\text { Referenced Table } 1 \text { in separate } \\
\text { book for fatigue data }\end{array}$ & $\begin{array}{l}\text { Only considered as part of more general creep/fatigue analysis. No } \\
\text { details of material properties for fatigue or any other design } \\
\text { applicaitons. This information is provided in a separate document } \\
\text { which is not accessible at this time }\end{array}$ \\
\hline g & $\begin{array}{l}\text { Collapse or breach of pressure } \\
\text { boundary due to corrosion, mass } \\
\text { transfer phenomenon, etc. }\end{array}$ & Yes & Section 1.2.2. & $\begin{array}{l}\text { Consideration given to specific effects of liquid sodium and radiation } \\
\text { embrittlement on material properties }\end{array}$ \\
\hline h & $\begin{array}{l}\text { Excessive deformation leading to } \\
\text { loss of functionality, due to creep } \\
\text { under steady load }\end{array}$ & Yes/No & Section 3.4. & Like NH only addresses issue of strain limits. Same limits as NH \\
\hline
\end{tabular}


Table 9.2.3.3-2 Monju: Failure Mechanisms

\begin{tabular}{|c|l|c|l|l|}
\hline ID\# & Definition / Criterion & Covered? & Location & Comment \\
\hline $\mathrm{i}$ & Creep rupture & No/Yes & Asada PVP paper & $\begin{array}{l}\text { Creep rupture is presumably used in setting high temperature design } \\
\text { allowables, but no information available on method; New design } \\
\text { procedure "without stress classification" employs Reference Stress } \\
\text { concept, which might be interpreted as addressing creep rupture } \\
\text { directly }\end{array}$ \\
\hline $\mathrm{j}$ & Creep buckling & No & & $\begin{array}{l}\text { Buckling is deliberately not considered; predominately pressure } \\
\text { vessels under tensile loading, similar case for internals components of } \\
\text { AGR. }\end{array}$ \\
\hline $\mathrm{k}$ & Enhanced creep & Yes & Section 3.4.2 & \\
\hline $\mathrm{l}$ & Creep-Fatigue & Yes & & See "f" above \\
\hline $\mathrm{m}$ & Accelerated creep rupture & No & & \\
\hline $\mathrm{n}$ & $\begin{array}{l}\text { Softening enhanced cyclic creep } \\
\text { rupture }\end{array}$ & No & & Records need to consider but offers no reccomendations \\
\hline & & No & & \\
\hline $\mathrm{p}$ & $\begin{array}{l}\text { Softening enhanced cyclic creep } \\
\text { deformation }\end{array}$ & Irradiation effects & & \\
\hline
\end{tabular}


Table 9.2.3.3-3 Monju: Failure Mechanisms

\begin{tabular}{|c|l|c|l|l|}
\hline ID\# & \multicolumn{1}{|c|}{ Definition / Criterion } & Covered? & Location & Comment \\
\hline q & weldments (strength, strain) & No & & $\begin{array}{l}\text { No specific references to weldments. May be information in } \\
\text { materials book which is not available }\end{array}$ \\
\hline r & $\begin{array}{l}\text { Multiaxial effects on creep, fatigue, } \\
\text { C-F }\end{array}$ & No & & See "q" above \\
\hline s & Local vs. distributed damage & Yes & Sections 3.4.2, 5.2.1, 5.5.3 & Part of creep/fatigue evaluation \\
\hline t & Thermal aging & No & & See "q" above \\
\hline u & Elastic follow-up & Yes & Section 3.5.3. & $\begin{array}{l}\text { Elastic analysis. Referred to extensively in piping analysis as part } \\
\text { of procedure for factorizing thermal stresses into primary and } \\
\text { secondary components. No specific rules given to implement } \\
\text { procedure. Much left to judgment }\end{array}$ \\
\hline V & Ratchet Fatigue & Yes & Asada PVP paper & New failure mechanism not considered elsewhere \\
\hline
\end{tabular}


Table 9.2.3.4-1 Monju: Design Criteria / Procedures

\begin{tabular}{|l|l|l|l|l|l|l|l|l|l|}
\hline & & & & & & & \\
\end{tabular}


Table 9.2.3.4-2 Monju: Design Criteria / Procedures

\begin{tabular}{|c|c|c|c|c|c|c|c|c|c|}
\hline 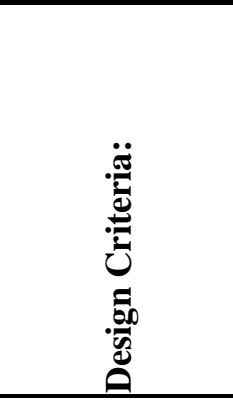 & 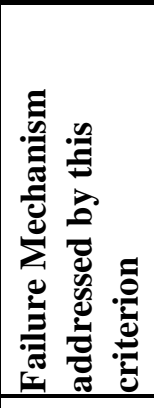 & 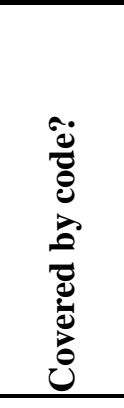 & 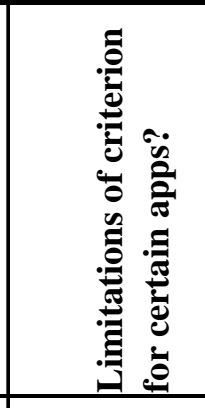 & 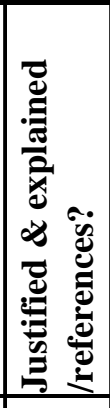 & 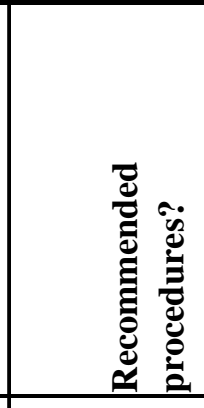 & 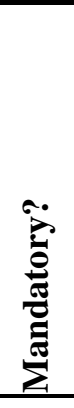 & 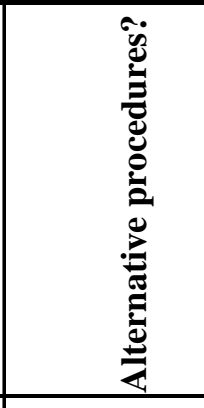 & 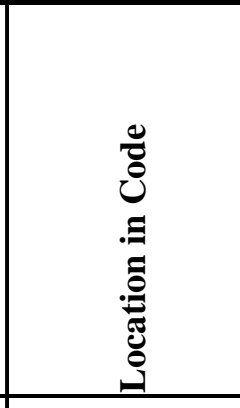 & 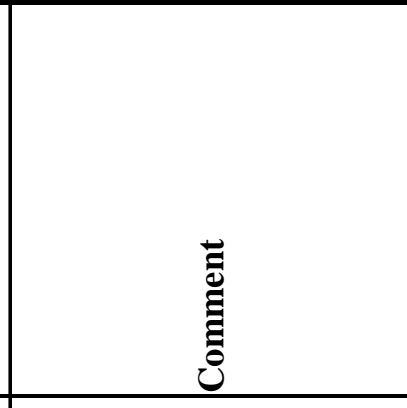 \\
\hline iv. Ratcheting & d & No/Yes & Not specified & No & No details & ? & No & Asada PVP paper & $\begin{array}{l}\text { constraint onextent, not } \\
\text { magnitude of stresses, in region } \\
\text { exceeding yield }\end{array}$ \\
\hline v. Fracture & e & No & No & No & No & No & & & \\
\hline vi. Fatigue & $\mathrm{f}$ & Yes. & No details & No. & Miner's rule. & Yes. & None apparent & Table 1 & $\begin{array}{l}\text { Fatigue addressed in creep- } \\
\text { fatigue criteria }\end{array}$ \\
\hline vii. Corrosion & g & Yes & Liquid sodium & No & No & Yes & No & 1.2 .2 & No specific methods \\
\hline
\end{tabular}


Table 9.2.3.4-3 Monju: Design Criteria / Procedures

\begin{tabular}{|c|c|c|c|c|c|c|c|c|c|}
\hline 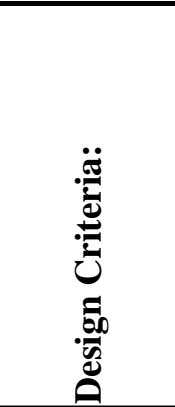 & 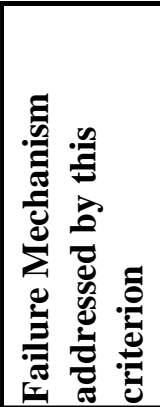 & 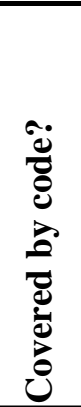 & 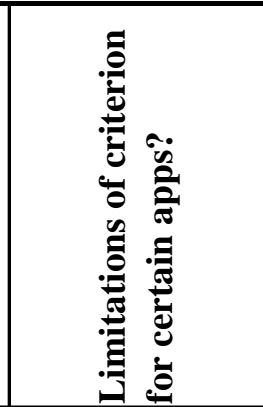 & 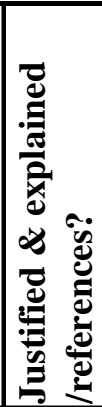 & 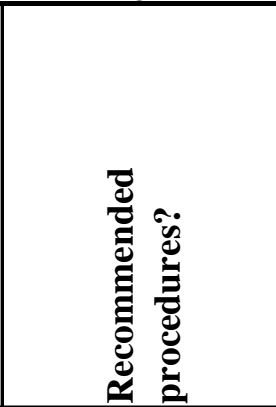 & 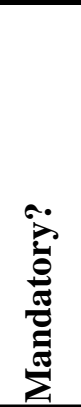 & 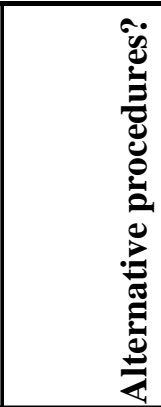 & 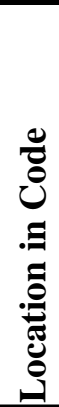 & 葛 \\
\hline $\begin{array}{c}\text { vii. } \\
\text { Corrosion } \\
\end{array}$ & $\mathrm{g}$ & No. & & & & & & & \\
\hline $\begin{array}{c}\text { viii. } \\
\text { Excessive } \\
\text { creep } \\
\text { deformation }\end{array}$ & $\mathrm{h}$ & Yes. & $\begin{array}{l}\text { Strain limitations, } \\
\text { not structural } \\
\text { deformation limits }\end{array}$ & Yes. & $\begin{array}{l}\text { Essentially the same } \\
\text { procdrue as NH }\end{array}$ & Yes. & no & & $\begin{array}{l}\text { Strain is limited to } 1 \%, 2 \% \text { \& } \\
5 \% \text {, AND stress intensity is } \\
\text { dictated by limiting strain to } \\
1 \%\end{array}$ \\
\hline $\begin{array}{l}\text { ix. Creep } \\
\text { buckling }\end{array}$ & h,b & No. & & & & & & & \\
\hline
\end{tabular}


Table 9.2.3.4-4 Monju: Design Criteria / Procedures

\begin{tabular}{|c|c|c|c|c|c|c|c|c|c|}
\hline 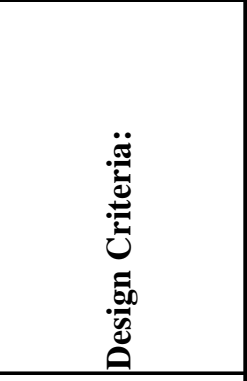 & 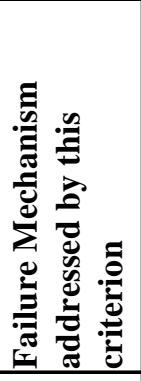 & 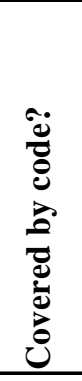 & 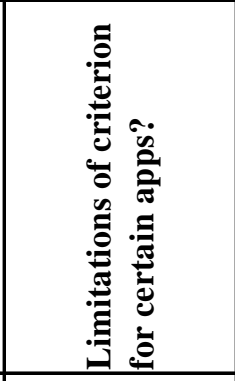 & 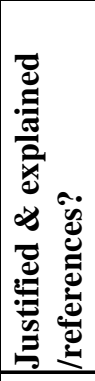 & 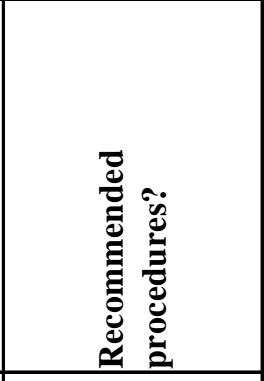 & 葛 & 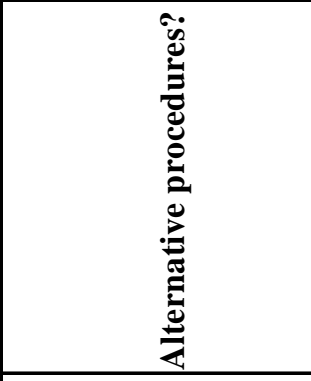 & 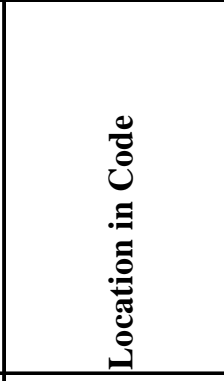 & 冚 \\
\hline x. Creep rupture & b,h,i & Yes? & $\begin{array}{l}\text { No specific details } \\
\text { on the nature of } \\
\text { creep rupture } \\
\text { failure mechanism. } \\
\text { Follows NH }\end{array}$ & no & $\begin{array}{l}\text { Follows NH practice; } \\
\text { new no-classification } \\
\text { procedure uses } \\
\text { Reference Stress } \\
\text { concept }\end{array}$ & Yes. & $\begin{array}{l}\text { Follows NH' new } \\
\text { classification free method } \\
\text { uses reference stress }\end{array}$ & Asada PVP paper & \\
\hline $\begin{array}{c}\text { xi. Enhanced } \\
\text { Creep Deformation }\end{array}$ & $\mathrm{j}$ & No & & & & & & & \\
\hline $\begin{array}{c}\text { xii. C-F } \\
\text { Interaction }\end{array}$ & $3 \mathrm{k}$ & Yes. & $\begin{array}{l}\text { Linear damage rule } \\
\text { limitations. }\end{array}$ & Yes. & Linear damage rule. & Yes. & no & Section 3.4.2 & \\
\hline
\end{tabular}


Table 9.2.3.4-5 Monju: Design Criteria / Procedures

\begin{tabular}{|c|c|c|c|c|c|c|c|c|c|}
\hline 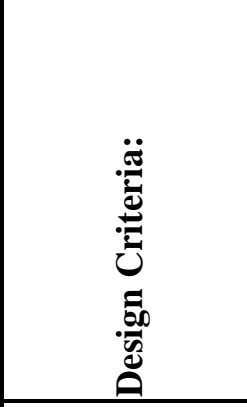 & 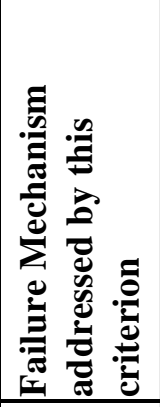 & 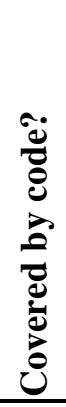 & 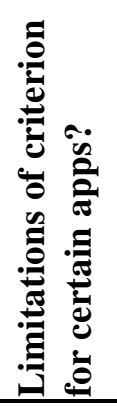 & 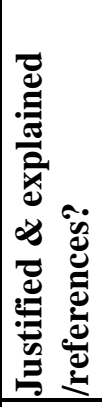 & 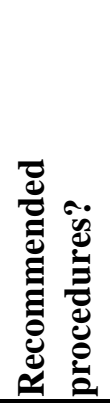 & 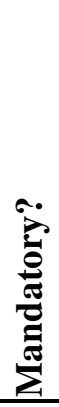 & 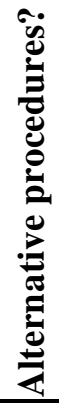 & 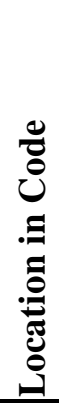 & 莣 \\
\hline $\begin{array}{l}\text { xiii. Accelerated } \\
\text { Cyclic Creep } \\
\text { Rupture }\end{array}$ & l & No & & & & & & & \\
\hline $\begin{array}{c}\text { xiv. Softening } \\
\text { Enhanced Cyc. } \\
\text { Creep } \\
\text { Deformation }\end{array}$ & $\mathrm{m}$ & No. & & & & & & & \\
\hline $\begin{array}{l}\text { xv. Softening } \\
\text { Enhanced Cyc. } \\
\text { Creep Rupture }\end{array}$ & $\mathrm{n}$ & No. & & & & & & & \\
\hline
\end{tabular}


Table 9.2.3.4-6 Monju: Design Criteria / Procedures

\begin{tabular}{|c|c|c|c|c|c|c|c|c|c|}
\hline 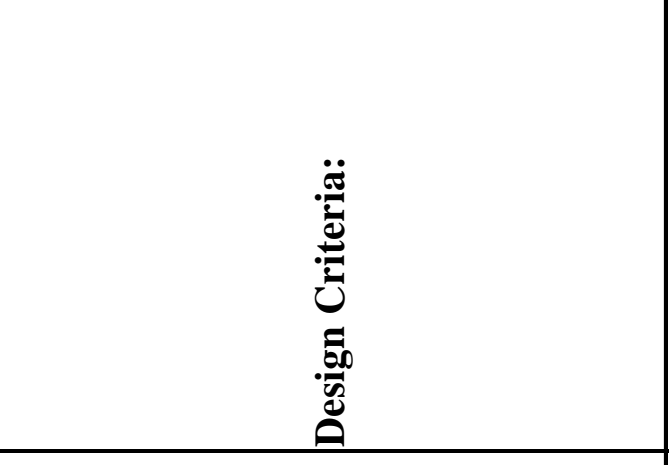 & 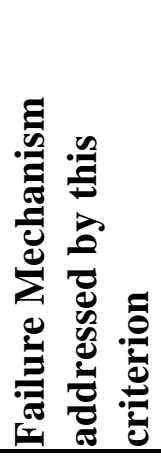 & 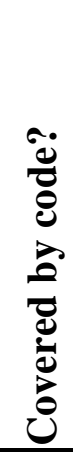 & 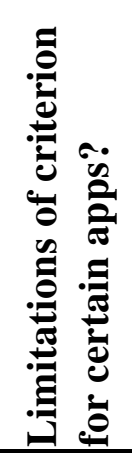 & 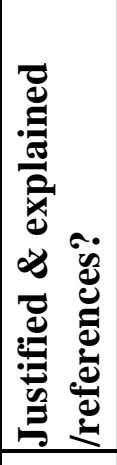 & 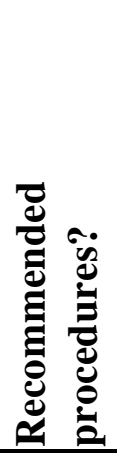 & 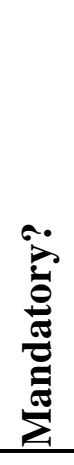 & 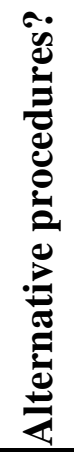 & 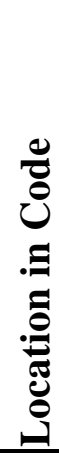 & 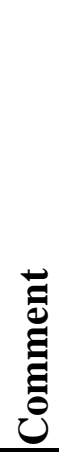 \\
\hline xvi. Irradiation induced swelling & 0 & No. & & & & & & & \\
\hline xvii. Irradiation induced creep & 0 & No. & & & & & & & \\
\hline $\begin{array}{l}\text { xviii. Plastic Flow localization due to } \\
\text { irradiation (dislocation tunneling) }\end{array}$ & 0 & No. & & & & & & & \\
\hline $\begin{array}{c}\text { xix. Ductility exhaustion due to irradiation } \\
\text { exposure }\end{array}$ & 0 & No. & & & & & & & \\
\hline $\begin{array}{l}\text { xx. Irradiation induced effects on fatigue, } \\
\text { and on C-F }\end{array}$ & 0 & No. & & & & & & & \\
\hline
\end{tabular}


Table 9.2.3.4-7 Monju: Design Criteria / Procedures

\begin{tabular}{|c|c|c|c|c|c|c|c|c|c|}
\hline 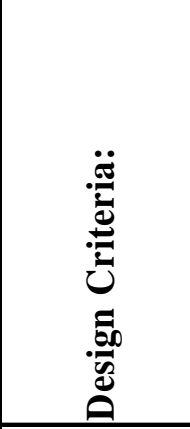 & 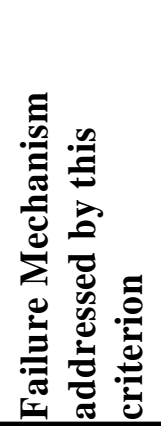 & 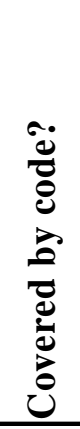 & 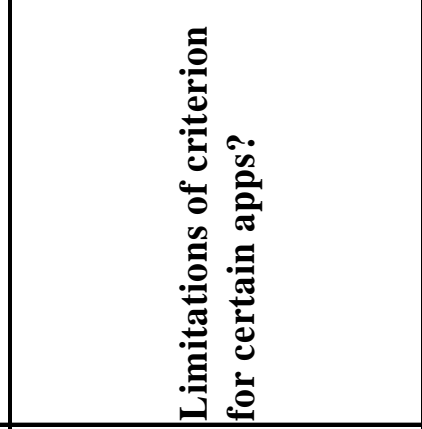 & 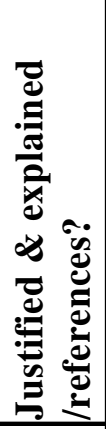 & 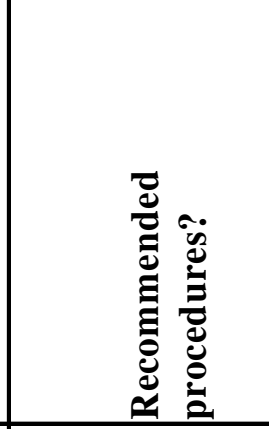 & 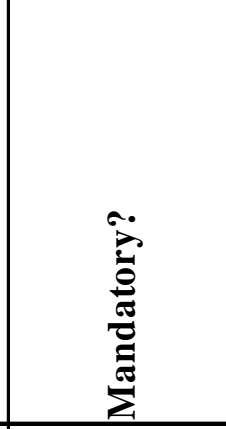 & 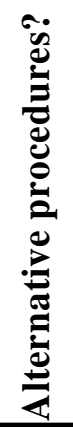 & 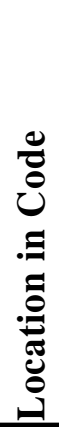 & 苞 \\
\hline $\begin{array}{c}\text { xxi. Local } \\
\text { vs. non-local } \\
\text { damage }\end{array}$ & $\mathrm{p}$ & No & & & & & & & \\
\hline $\begin{array}{c}\text { xxii. } \\
\text { Thermal } \\
\text { aging effects } \\
\text { on creep, } \\
\text { fatigue, limit } \\
\text { load }\end{array}$ & $q$ & No & & & & & & & \\
\hline $\begin{array}{c}\text { xxiii. } \\
\text { Elastic } \\
\text { Follow-up }\end{array}$ & $r$ & Yes. & $\begin{array}{l}\text { Elastic followup considered } \\
\text { explicitly in determination of } \\
\text { primary and secondary } \\
\text { components of stress, especially } \\
\text { in piping applications. }\end{array}$ & no & $\begin{array}{l}\text { Standard method of } \\
\text { calculating elastic } \\
\text { followup provied }\end{array}$ & $\begin{array}{l}\text { Yes, for } \\
\text { determination of } \\
\text { strain range for } \\
\text { C-F analysis. }\end{array}$ & & & \\
\hline
\end{tabular}




\subsubsection{RCC-MR}

The RCC-MR French Code review was conducted using the October 2007 English version of the Code. Since October 2007, some incremental improvements/changes and additions to the Code were summarized in PVP2008-61527 paper without much detail. The changes are listed below and quoted from the PVP2008 paper:

- Enlargement of the scope of the code to be applicable not only to high temperature structures but also to the ITER vacuum vessel.

- Adaptation of the code to the European standardization, making use of the Harmonized European Standards.

- Improvement of the creep-fatigue rules for shells and pipes.

- Development of the "class 2 box" structures directly applicable to the ITER vacuum vessel.

- Introduction of a specific appendix dealing with specific fabrication requirements of the ITER vacuum vessel.

- Extension of the scope of subsections devoted to bolts

- Development of the chapter on laser welding

- Introduction of requirements in line with the new European Pressure Equipment decree and its French declination to nuclear equipment (ESPN) which is mandatory for a French site construction.

Most of the procedures' changes do not address the simplified design rules. The paper does not reveal any information that may be useful to this study. The paper mentions that an improved creep-fatigue design rule was established, but it does not reveal what that rule is.

This review used RB3000 section of the Code which addresses high temperature applications, October 2007 release. It briefly describes the failure mechanisms but provides a fairly detailed description of the design procedures for various design criteria. While Task 9.2 is not intended to compare International Codes, one cannot help but mention the great similarity between RCC-MR and the ASME NH Codes in terms of design criteria and procedures.

\subsubsection{RCC-MR - High Temperature}

Two main definitions / criteria were identified in the Ideal ETD Code: a High Temperature Limit, and a Criterion for Insignificant Creep. Table 9.2.4.1 below summarizes this information. The 'High temperature limit' is summarized in ID\# 'a', the definition is 'covered' by RCC-MR, the appropriate location in the Code is indicated, as well as the inclusion of appropriate comments. The creep rupture curves are provided as a function of time and temperature for various materials. 
Similarly, the insignificant creep definition is covered, with details regarding the definition summarized in the comment section.

The Code provides two tests for establishing if the conditions warrant the assumption of negligible creep. Test 1 checks if the maximum temperature is less than a threshold value established in A3.31 appendix. Test 2 states "For a component or part of a component, the creep is said to be negligible over the operating period corresponding to the loadings for which compliance with level A and C criteria is required if, both conditions of test 1 not being satisfied for the total operating period, at least one is met when the level D criterion loadings are ignored." The description of Test 2 is ambiguous and requires further explanation.

The RCC-MR Code, just like the NH Code, contains appendices for materials that are allowed in the Code, and it is speculated that these appendices contain information such as isochronous curves as well as threshold temperatures beyond which creep becomes significant. These appendices were not made available for this study.

\subsubsection{RCC-MR - Design Loads}

RCC-MR does a very good job in defining stress classification, stress intensities. Tables 9.2.4.2-1 and 9.2.4.2-2 summarize information relevant to 'Design Loads' for an Ideal ETD Code, including the Definition / Criterion, whether the Definition is 'Covered', the location in the Code where the Definition is addressed, and relevant comments. Below are highlights of design load definitions:

- The guideline for defining the cycles is very brief and provides general rules for establishing cycles. It does not provide specific information that is useful to the designer.

- Primary and secondary stress classification is well documented, including the definition of various stress components such as membrane, bending and peak.

- The various load categories are well established and defined. RCC-MR also provides a clear and concise list of design rules that must be applied for each load category.

- Elastic follow-up is very well described, and design rules take it into consideration and account for it in the established procedures by providing elastic follow-up factors.

- Stress classification is presented in Table RB3324.31 for a limited set of components such as vessels and flat heads.

- The stress/load classification is limited to symmetric structures and loading, and there are no guidelines for other application cases. 


\subsubsection{RCC-MR - Failure Mechanisms}

The RCC-MR Section RB3100 describes the general design rules for class 1 components under two main failure mechanisms, which it calls damages: $\mathrm{P}$ type damage and S type damage. The $\mathrm{P}$ type damage "can result from a steadily and regularly increasing loading or a constant loading”. This includes immediate excessive deformation, plastic instability, time dependent excessive deformation, plasticity, fracture, and elastic or elastic-plastic instability. The $\mathrm{S}$ type damage refers to all the failure mechanisms due to repeated loading, such as progressive deformation (ratcheting) and fatigue cracking.

Tables 9.2.4.3-1 \& 9.2.4.3-2 contain a summary of the failure mechanisms addressed in RCC-MR procedure, and they highlight those that are not addressed in it. In these tables, three possibilities exist for response to the column labeled 'Covered?', "Yes”, "No", and "Yes/No", with the latter requiring an explanation. "Yes/No" indicates that the Code does not explicitly address the failure mechanism or does in a limited way or circumstances; but the Code does address the mechanism indirectly to some extent. The "Yes/No" also may be due to unavailability of relevant information to the review team.

The following are observations regarding the failure mechanisms stated in the RCC-MR Code:

- Corrosion, mass transfer phenomenon are not addressed

- Softening enhanced cyclic creep rupture is not addressed

- Irradiation effects are not addressed.

- Thermal aging is addressed by dictating scale factors to be applied to $S_{m}$ in calculating creep strain.

- There is no distinction between excessive deflection and excessive deformation. The limit on deflection is dictated by the applicants

- Strain limits are $1 \%$ for average through a section of interest and $2 \%$ for average plus bending. There does not seem to be a limit of peak strains. These limits are cut by a factor of 2 for welds.

\subsubsection{RCC-MR - Design Criteria/Procedures}

Tables 9.2.4.4-1 through 9.2.4.4-5 summarize the various design criteria of an Ideal ETD Code and how RCC-MR addresses them or not. The first column indicates the design criteria, and the failure mechanism addressed by the criterion is listed in the second column; whether or not the RCC-MR Procedure covers the design criteria is indicated in the third column. Limitations of the criteria, justification \& explanation / references for the design criteria, and recommended procedures are also listed, including whether or not they are mandatory. Alternative procedures are summarized if such information is available, including the locations in the Code that address the criteria. Finally, comments are included as appropriate. 
The RCC-MR does not address the fast fracture, irradiation or corrosion effects. It addresses the elastic follow-up in significant creep procedures by dictating certain elastic follow-up factors based on application conditions. The effect of multi-axial loading on the creep is addressed by adjusting the stress tensors as provided in RB3226.1. The thermal aging effects are handled by aging factors documented in A3.51; the aging factor is applied to $S_{\mathrm{m}}$. Also, temperature and time conditions likely to produce a significant aging degradation are given in A3.31.

- The following are highlights of the design procedures:

- For P type loading (non-cyclic), RCC-MR allows the use of elastic, limit analysis or elastoplastic analysis methods with negligible creep, but only elastic and limit analyses for significant creep.

- For S type loading (cyclic) only elastic and elasto visco plastic analyses are allowed.

- Limit analysis methods must not be combined with any other method.

- The rules do not mandate one analysis method over another.

- The design limits (for example inelastic strain in the core, or strain limited allowable stress intensity) depend on what analysis method is used, i.e. elastic, elastoplastic, limit analysis, or experimental.

- While not labeled as core stress, effective membrane stress which describes the state of stress at the midsection of a structure is used to predict the inelastic strain in the structure in $\mathrm{S}$ type damage..

- Same type of analysis must be used for all parts of the component (i.e. elastic, inelastic).

- Rules of stress classification and corresponding strain analysis are based on Bree type problem. There does not seem to be any information on how other loading or component configurations should be dealt with.

- There are no specific buckling procedures specified, but elastic and inelastic methods are allowed.

- Local primary stress criterion is established in RB3251.11

- Cyclic loading elastic analysis procedures are established:

o Detailed formulation of effective primary and secondary stress components are provided. A correction factor for overstress during short duration is used to modify the effective primary stress (RB3261.1112.2).

o Efficiency index, which depends on secondary stress levels, is used to modify the effective primary stress as function of secondary stress. The resulting modified effective primary stresses are then limited to $1.3 \mathrm{Sm}$ (membrane) and 1.95Sm (membrane plus bending); this guarantees the average strain level to be below $1 \%$ and the bending plus average strain to be below $1.7 \%$ for austenitic steels.

- In predicting strain range (for fatigue analysis), the procedure includes a correction for triaxiality (RB3261.123). Elastic method has rigorous procedures for predicting strain range which is composed of four individual strain range components based on cyclic stress-strain curve (RB3261.123).

- For creep rupture usage fraction computation, the effective stress is divided by 0.9 factor, but no justification is given. 
In general, the design criteria or procedures are not justified, and no references are provided to support them. The Code's strong point is the explicit and detailed description of the mathematical procedures needed to calculate certain quantities like strain range (RB3227.9) and stress range (RB3224.45). The code provides the flexibility of using inelastic analysis for most of the design criteria. 
Table 9.2.4.1 RCC-MR: High Temperature

\begin{tabular}{|c|c|c|l|l|}
\hline ID \# & Definition/Criterion & Covered? & Location & Comments \\
\hline & High temperature limit & YES & $\begin{array}{l}\text { RB3216.1 } \\
\text { Test 1\&2 } \\
\text { A3.31 }\end{array}$ & $\begin{array}{l}\text { Sr Creep Rupture Curves as function } \\
\text { of time and temperature in Appendix } \\
\text { A3.31 }\end{array}$ \\
\hline b & $\begin{array}{c}\text { Criterion or definition of temperature } \\
\text { limit for insignificant creep effects }\end{array}$ & YES & $\begin{array}{l}\text { RB3216.11 } \\
\text { Test 1\&2 }\end{array}$ & \\
\hline
\end{tabular}

Table 9.2.4.2-1 RCC-MR Procedure: Design Loads

\begin{tabular}{|c|c|c|c|c|}
\hline ID \# & Definition / Criterion & Covered? & Location & Comment \\
\hline $\mathrm{a}$ & $\begin{array}{l}\text { Guidelines for load } \\
\text { histogram \& cycle } \\
\text { definition }\end{array}$ & Yes & $\begin{array}{l}\text { RB3222 } \\
\text { RB3263 }\end{array}$ & $\begin{array}{l}\text { RB3222- general definition } \\
\text { RB3263: General guideline for definition of load cycles }\end{array}$ \\
\hline $\mathrm{b}$ & Glossary of terms & Yes & RB3100 & $\begin{array}{l}\text { General definitions of P and S type loads and heir } \\
\text { categories and load criteria/levels }\end{array}$ \\
\hline C & $\begin{array}{l}\text { Definition of stress } \\
\text { intensities }\end{array}$ & Yes & RB3200 & Definition of stress intensities \\
\hline d & $\begin{array}{l}\text { Definition of strain } \\
\text { intensities }\end{array}$ & Yes & RB3227 & Defines strain ranges and not intensities \\
\hline e & $\begin{array}{l}\text { Definition of primary vs } \\
\text { secondary stresses }\end{array}$ & Yes & RB3224.3 & Similar to NH code \\
\hline $\mathrm{f}$ & $\begin{array}{l}\text { Definition of membrane, } \\
\text { bending, peak stresses }\end{array}$ & Yes & $\begin{array}{l}\text { Table RB3324.31 } \\
\text { RB3224.1 } \\
\text { RB3224.3 }\end{array}$ & $\begin{array}{l}\text { Table RB3324.31:classification of stresses in vessels (a } \\
\text { few typical cases) } \\
\text { RB3224.1:Defines stress terms } \\
\text { RB3224.2: Defines stress classifications under elastic } \\
\text { analysis (primary, secondary, linear, nonlinear...etc.) }\end{array}$ \\
\hline g & $\begin{array}{l}\text { Procedure for stress } \\
\text { linearization }\end{array}$ & Yes & RB3224.14 & Very general. Not clear \\
\hline
\end{tabular}


Table 9.2.4.2-2 RCC-MR Procedure: Design Loads

\begin{tabular}{|c|c|c|c|c|}
\hline ID & $\begin{array}{l}\text { Definition / } \\
\text { Criterion }\end{array}$ & Covered? & Location & Comment \\
\hline h & $\begin{array}{l}\text { Differentiated load } \\
\text { categories: }\end{array}$ & Yes & $\begin{array}{l}\text { RB3130- } \\
\text { RB3160 }\end{array}$ & $\begin{array}{l}\text { RB3150- describes: } \\
\text { Level A Load Criteria: The aim of level A criteria is to protect the equipment against the following damage: } \\
\text { immediate or time-dependent excessive deformation, immediate or time-dependent plastic instability, } \\
\text { time-dependent fracture, elastic or elastoplastic instability, immediate or time-dependent, } \\
\text { progressive deformation, fatigue. } \\
\text { The respect of level A criteria guarantees the level of safety required with regard to these types of damage throughout the life of the } \\
\text { equipment, for operation as specified. } \\
\text { RB } 3152 \text { Level B criteria } \\
\text { The ASME code introduces a level B into the design of pressure retaining enclosures by introducing a certain tolerance into the } \\
\text { design internal pressure value. This provision does not figure in this Code. } \\
\text { RB } 3153 \text { Level C criteria } \\
\text { The aim of level C criteria is to protect the equipment against the following damage: } \\
\text { immediate or time-dependent excessive deformation, immediate or time-dependent plastic instability, } \\
\text { time-dependent fracture, elastic or elastoplastic instability, immediate or time-dependent. } \\
\text { RB } 3154 \text { Level D criteria } \\
\text { The aim of level D criteria is to protect the equipment against the same damage as level C but with lower safety margins. } \\
\text { It will not always be possible to return to service an item of equipment having been subjected to a loading only limited by level D } \\
\text { criteria. } \\
\text { RB } 3155 \text { Level } 0 \text { criteria } \\
\text { The regulations relating to pressure-retaining vessels, define criteria linked with a fictitious design condition. This is closely } \\
\text { connected to the need to define a design pressure for testing purposes. This type of criteria level is not envisaged in this Code. In the } \\
\text { case of pressure retaining equipment, the regulations in force shall be applied. }\end{array}$ \\
\hline & design loads & Yes & RB3170 & Specifications of loads must be provided by applicant \\
\hline & normal service loads & Yes & RB3130 & SF1 category \\
\hline & frequent abnormal loads & Yes & RB3130 & SF2 category \\
\hline & $\begin{array}{r}\text { infrequent abnormal } \\
\text { loads }\end{array}$ & Yes & RB3130 & SF3 type category \\
\hline & limiting fault loads & Yes & RB3130 & SF4 type category \\
\hline & test loads & Yes & RB3130 & Considered in SF1-SF4 \\
\hline $\mathbf{i}$ & $\begin{array}{r}\text { Flow chart for design by } \\
\text { analysis or } \\
\text { rule/procedure }\end{array}$ & Yes & RB3200 & RB3200- contains all rules/procedures for design by analysis \\
\hline $\mathbf{j}$ & $\begin{array}{r}\text { Yield function } \\
\text { requirements (e.g. Tresca } \\
\text { vs. Mises) }\end{array}$ & Yes & RB3224.4 & $\begin{array}{l}\text { Allows maximum shear \& octahedral shear theories } \\
\text { Provides explicit instructions on evaluating stress intensity scalars. }\end{array}$ \\
\hline $\mathbf{k}$ & Elastic follow-up & Yes & RB3225 & General definition of elastic follow-up \\
\hline
\end{tabular}


Table 9.2.4.3-1 RCC-MR: Failure Mechanisms

\begin{tabular}{|c|c|c|c|c|}
\hline ID \# & Definition/Criterion & Covered? & Location & Comment \\
\hline $\mathbf{a}$ & $\begin{array}{l}\text { Limit load collapse, under a single load } \\
\text { application. }\end{array}$ & $\mathrm{Y}$ & RB3121.2 & Plastic instability \\
\hline b & $\begin{array}{l}\text { Excessive deformation, limiting functionality, } \\
\text { under a single load application, below the limit } \\
\text { load. }\end{array}$ & $\mathrm{Y}$ & RB3121.1 & RB3121.1: immediate collapse \\
\hline c & $\begin{array}{l}\text { Structural instability or buckling, under a single } \\
\text { load application. }\end{array}$ & $\mathrm{Y}$ & RB3123 & $\begin{array}{l}\text { RB3123.1- load controlled } \\
\text { RB3123.2- strain controlled }\end{array}$ \\
\hline d & $\begin{array}{l}\text { Progressive collapse by ratcheting under cyclic } \\
\text { load. }\end{array}$ & $\mathrm{Y}$ & RB122.1 & Could include creep deformation \\
\hline $\mathbf{e}$ & Non-ductile fracture & $\mathrm{Y}$ & RB3124 & $\begin{array}{l}\text { RB3124: defines fast fracture. does not contain rules for prevention of fast } \\
\text { fracture. This protection is obtained by manufacturing methods which allow } \\
\text { excessive plastic elongation to be avoided and by the choice of materials. }\end{array}$ \\
\hline f & Fatigue failure (nucleation on order of $5 \mathrm{~mm}$ )) & $\mathrm{Y}$ & RB3122.2 & Includes creep \\
\hline g & $\begin{array}{l}\text { Collapse or breach of pressure boundary due to } \\
\text { corrosion, mass transfer phenomenon, etc. }\end{array}$ & $\mathrm{Y} / \mathrm{N}$ & $\begin{array}{l}\text { RB3110 } \\
\text { RB3191 }\end{array}$ & $\begin{array}{l}\text { RB3110: indicates that "other rules must be applied, but does not reference } \\
\text { any of them. } \\
\text { RB3191Mandates additional thickness for corrosion and erosion }\end{array}$ \\
\hline $\mathbf{h}$ & $\begin{array}{l}\text { Excessive deformation leading to loss of } \\
\text { functionality, due to creep under essentially } \\
\text { steady load }\end{array}$ & $\mathrm{Y}$ & RB3121.3 & Time dependent Excessive deformation \\
\hline $\mathbf{i}$ & Creep rupture & $\mathrm{Y}$ & RB121.3 & $\begin{array}{l}\text { Time dep. Excessive def. } \\
\text { Creep rupture usage criterion }\end{array}$ \\
\hline $\mathbf{j}$ & Creep buckling & $\mathrm{Y}$ & $\begin{array}{l}\text { RB } 3121.6 \\
\text { RB } 3123.3\end{array}$ & $\begin{array}{l}\text { RB 3121.6: definition } \\
\text { RB 3123.3: time dep. buckling }\end{array}$ \\
\hline
\end{tabular}


Table 9.2.4.3-2 RCC-MR: Failure Mechanisms

\begin{tabular}{|c|c|c|c|c|}
\hline ID \# & Definition/Criterion & Covered? & Location & Comment \\
\hline $\mathbf{k}$ & Enhanced creep & $\mathrm{Y} / \mathrm{N}$ & RB3122.1 & $\begin{array}{l}\text { Progressive deformation-this is not well spelled out. Lumped under } \\
\text { progressive def. }\end{array}$ \\
\hline $\mathbf{l}$ & Creep-fatigue & $\mathrm{Y} / \mathrm{N}$ & RB3122.1 & $\begin{array}{l}\text { Progressive deformation-this is not well spelled out. Lumped under } \\
\text { progressive def. }\end{array}$ \\
\hline $\mathbf{m}$ & Accelerated creep rupture & $\mathrm{Y} / \mathrm{N}$ & RB3122.1 & $\begin{array}{l}\text { Progressive deformation- - this is not well spelled out. Lumped under } \\
\text { progressive def. }\end{array}$ \\
\hline $\mathbf{n}$ & Softening enhanced cyclic creep rupture & $\mathrm{Y} / \mathrm{N}$ & RB3122.1 & $\begin{array}{l}\text { Progressive deformation- - this is not well spelled out. Lumped under } \\
\text { progressive def. }\end{array}$ \\
\hline $\mathbf{0}$ & Softening enhanced cyclic creep deformation & $\mathrm{Y} / \mathrm{N}$ & RB3122.1 & $\begin{array}{l}\text { Progressive deformation—-this is not well spelled out. Lumped under } \\
\text { progressive def.. }\end{array}$ \\
\hline $\mathbf{p}$ & Irradiation effects & $\mathrm{Y} / \mathrm{N}$ & $\begin{array}{l}\text { RB3110 } \\
\text { RB3140 }\end{array}$ & General disclaimer that such effects must be considered \\
\hline $\mathbf{q}$ & Weldments (strength, strain) & $\mathrm{Y}$ & $\begin{array}{l}\text { RB3192 } \\
\text { RB3193 }\end{array}$ & $\begin{array}{l}\text { RB3192: General disclaimer: welds/attachments must be checked. RB3193: } \\
\text { general disclaimer: incompatible mat'l props. }\end{array}$ \\
\hline $\mathbf{r}$ & Multi-axial effects on creep, fatigue, C-F & $\mathrm{Y} / \mathrm{N}$ & $\begin{array}{l}\text { RB3226.1 } \\
\text { RB3261.12 }\end{array}$ & $\begin{array}{l}\text { RB3226.1 } \overline{\sigma_{\mathrm{j}}} \text { can be replaced by } 0.867 \overline{\sigma_{\mathrm{j}}}+0.133 \mathrm{tr}_{\mathrm{j}} \text { in creep analysis; } \\
\text { triaxiality strain range factor for fatigue analysis }\end{array}$ \\
\hline $\mathbf{s}$ & Local vs. non-local damage & $\mathrm{Y} / \mathrm{N}$ & & $\begin{array}{l}\text { Design criteria cover and procedures do address this issue, but the failure } \\
\text { mechanism section of the code (RB3100) does not treat this issue. }\end{array}$ \\
\hline $\mathbf{t}$ & Thermal aging & $\mathrm{Y} / \mathrm{N}$ & $\begin{array}{l}\text { RB3170 } \\
\text { RB } 3195\end{array}$ & $\begin{array}{l}\text { General disclaimer: select material with temp/hrs preventing aging. RB3195: } \\
\text { A3.31 has time/temp conditions for thermal aging; aging factor in A3.51; factor } \\
\text { applied to allowable stress } S_{m}\end{array}$ \\
\hline
\end{tabular}


Table 9.2.4.4-1 RCC-MR: Design Criteria

\begin{tabular}{|c|c|c|c|c|c|c|c|c|c|}
\hline 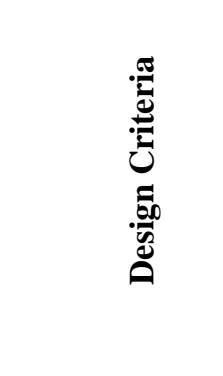 & 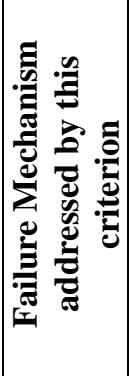 & 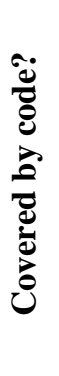 & 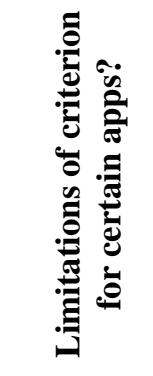 & 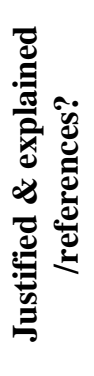 & 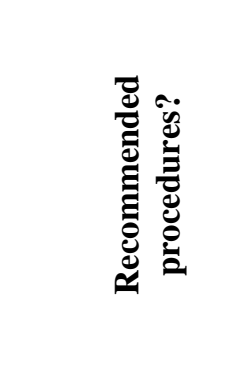 & 总 & 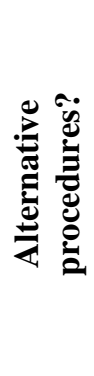 & 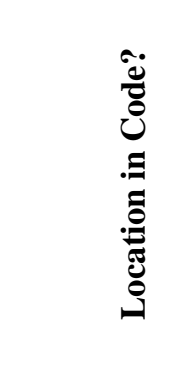 & 离 \\
\hline i. Limit Load & $\mathrm{a}$ & $\mathrm{Y}$ & & $\mathrm{N}$ & Elastic/inelastic & $\mathrm{N}$ & & $\begin{array}{l}\text { RB3251.12 } \\
\text { RB3251.13 }\end{array}$ & $\begin{array}{l}\text { Load Multiplication factors used for } \\
\text { inelastic method }\end{array}$ \\
\hline $\begin{array}{l}\text { ii. Excessive } \\
\text { deflection }\end{array}$ & b & $\mathrm{Y}$ & & $\mathrm{N}$ & Elastic/inelastic & $\mathrm{N}$ & & RB3252 & strain limited stress. \\
\hline $\begin{array}{l}\text { iii. } \\
\text { Buckling }\end{array}$ & C & $\mathrm{Y}$ & & $\mathrm{N}$ & Elastic/inelastic & $\mathrm{N}$ & & $\begin{array}{l}\text { RB3271, } \\
\text { RB3272 }\end{array}$ & $\begin{array}{l}\text { Buckling with insignificant creep \& } \\
\text { significant creep }\end{array}$ \\
\hline $\begin{array}{l}\text { iv. } \\
\text { Ratcheting }\end{array}$ & d & $\mathrm{Y}$ & $\begin{array}{l}\text { Complex } \\
\text { load and } \\
\text { geom. }\end{array}$ & $\mathrm{N}$ & Elastic/inelastic & $\mathrm{N}$ & & $\begin{array}{l}\text { RB3261.11 } \\
\text { RB3261.21 }\end{array}$ & $\begin{array}{l}.11 \text {-Progressive deformation-elastic } \\
.21 \text { - Progressive deformation-elasto-plastic }\end{array}$ \\
\hline v. Fracture & e & $\mathrm{N}$ & & $\mathrm{N}$ & & & & & $\begin{array}{l}\text { The code states it does not cover this } \\
\text { criterion. }\end{array}$ \\
\hline
\end{tabular}


Table 9.2.4.4-2 RCC-MR: Design Criteria

\begin{tabular}{|c|c|c|c|c|c|c|c|c|c|}
\hline 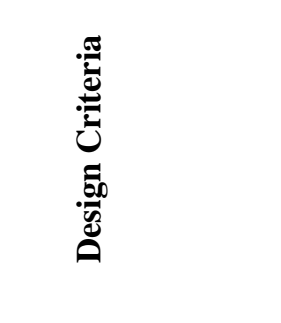 & 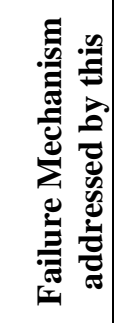 & 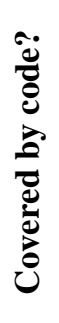 & 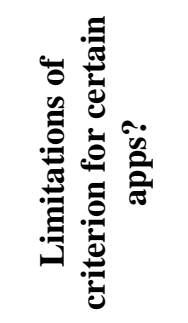 & 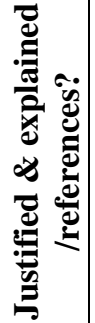 & 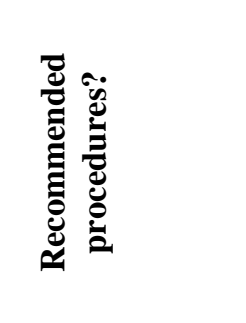 & 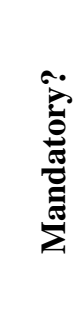 & 苞 & 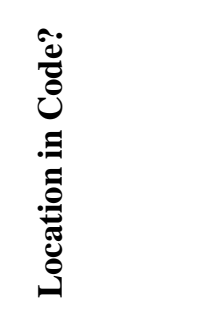 & ن̈ \\
\hline vi. Fatigue & $\mathrm{f}$ & $\mathrm{Y}$ & $\begin{array}{l}\text { Mean stress } \\
\text { effect in } \\
\text { HCF }\end{array}$ & $\mathrm{N}$ & Elastic/inelastic & $\mathrm{N}$ & & $\begin{array}{l}\text { RB3261.12 } \\
\text { RB3261.13 } \\
\text { Rb3262.123/4 } \\
\text { RB3262.2 }\end{array}$ & $\begin{array}{l}\text { RB3261.12- no geom.. discont. } \\
\text { RB3261.13 - geom.. discont. (zero rad)- } \\
\text { ELASTIC } \\
\text { RB3262.123/4- fatigue - usage fraction- } \\
\text { ELASTIC } \\
\text { RB3262.2-Elasto-visco-plast. }\end{array}$ \\
\hline $\begin{array}{l}\text { vii. } \\
\text { Corrosion }\end{array}$ & g & $\mathrm{N}$ & & & & & & & $\begin{array}{l}\text { RB3000 does not cover corrosion. states this } \\
\text { criterions is covered by other rules but does not } \\
\text { state what they are. }\end{array}$ \\
\hline $\begin{array}{l}\text { viii. } \\
\text { Excessive creep } \\
\text { deformation }\end{array}$ & h & $\mathrm{Y}$ & & $\mathrm{N}$ & Elastic/inelastic & $\mathrm{N}$ & & RB3262 & \\
\hline $\begin{array}{l}\text { ix. Creep } \\
\text { buckling }\end{array}$ & $\mathrm{j}$ & $\mathrm{Y}$ & & $\mathrm{N}$ & Elastic/inelastic & $\mathrm{N}$ & & RB3272 & $\begin{array}{l}\text { Buckling with creep. (RB3272 doc. is not } \\
\text { available). }\end{array}$ \\
\hline x. Creep rupture & b, h, i & $\mathrm{Y}$ & & $\mathrm{N}$ & Damage rule & $\mathrm{Y}$ & & RB3262.122 & RB3262.122 Creep rupture usage fraction \\
\hline $\begin{array}{l}\text { xi. Enhanced } \\
\text { Creep } \\
\text { Deformation }\end{array}$ & $\mathrm{k}$ & ?? & & & Elastic/inelastic & $\mathrm{N}$ & & RB3262.1 \&2 & \\
\hline
\end{tabular}


Table 9.2.4.4-3 RCC-MR: Design Criteria

\begin{tabular}{|c|c|c|c|c|c|c|c|c|c|}
\hline 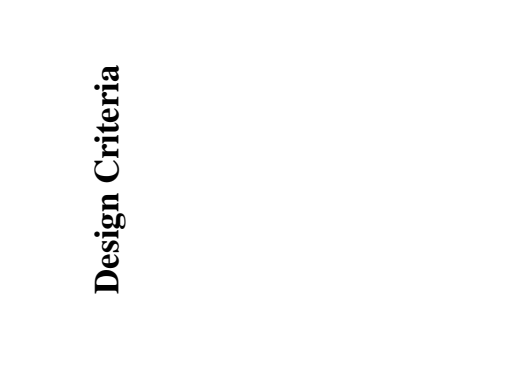 & 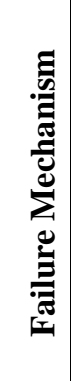 & 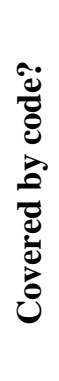 & 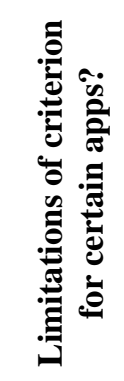 & 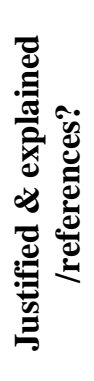 & 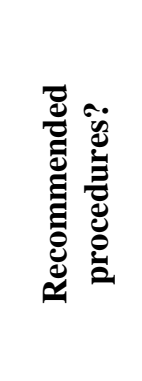 & 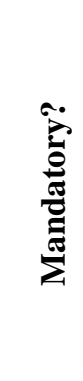 & 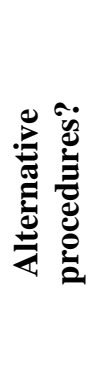 & 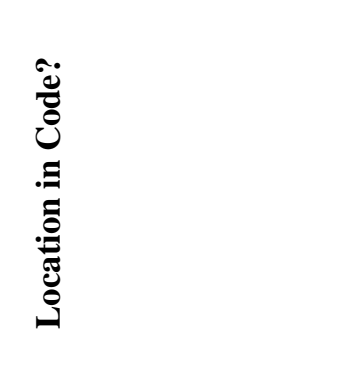 & 葛 \\
\hline xii. C-F Interaction & 1 & $\mathrm{Y}$ & $\begin{array}{l}\text { Linear } \\
\text { damage } \\
\text { rule }\end{array}$ & $\mathrm{N}$ & $\begin{array}{l}\text { Time/cy } \\
\text { cle } \\
\text { fraction } \\
\text { Elastic/ } \\
\text { inelastic }\end{array}$ & $\mathrm{N}$ & & $\begin{array}{l}\text { RB3262.12-elastic } \\
\text { RB3262.22-elast-visco- } \\
\text { plast }\end{array}$ & $\begin{array}{l}\text { Appendix A11- } \\
\text { guidelines for elast- } \\
\text { visco-plastic analysis }\end{array}$ \\
\hline $\begin{array}{c}\text { xiii. Accelerated Cyclic Creep } \\
\text { Rupture }\end{array}$ & $\mathrm{m}$ & $\mathrm{N}$ & & & & & & & \\
\hline $\begin{array}{l}\text { xiv. Softening Enhanced Cyc. } \\
\text { Creep Deformation }\end{array}$ & 0 & $\mathrm{~N}$ & & $\mathrm{~N}$ & & & & & \\
\hline $\begin{array}{l}\text { xv. Softening Enhanced Cyc. } \\
\text { Creep Rupture }\end{array}$ & $\mathrm{n}$ & $\mathrm{N}$ & & & & & & & \\
\hline xvi. Irradiation induced swelling & $\mathrm{p}$ & $\mathrm{N}$ & & & & & & & \\
\hline xvii. Irradiation induced creep & $\mathrm{p}$ & $\mathrm{N}$ & & & & & & & \\
\hline
\end{tabular}


Table 9.2.4.4-4 RCC-MR: Design Criteria

\begin{tabular}{|c|c|c|c|c|c|c|c|c|c|}
\hline 胥 & 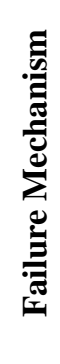 & 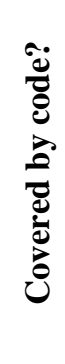 & 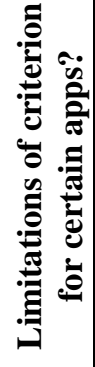 & 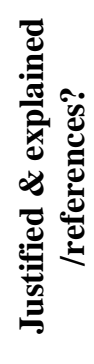 & 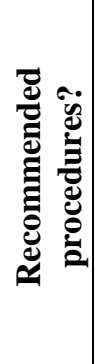 & 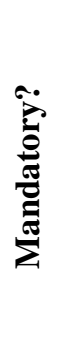 & 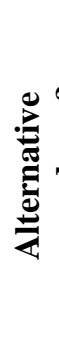 & 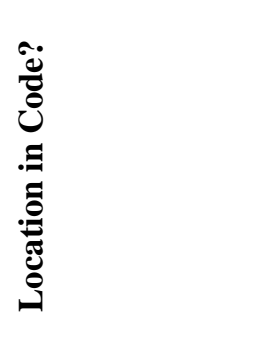 & ن \\
\hline \begin{tabular}{|c} 
xviii. \\
Plastic Flow \\
localization \\
due to \\
irradiation \\
(dislocation \\
tunneling)
\end{tabular} & $\mathrm{p}$ & $\mathrm{N}$ & & & & & & & \\
\hline $\begin{array}{l}\text { xix. Ductility } \\
\text { exhaustion } \\
\text { due to } \\
\text { irradiation } \\
\text { exposure }\end{array}$ & $\mathrm{p}$ & $\mathrm{N}$ & & & & & & & \\
\hline $\begin{array}{l}\text { xx. Irradiation } \\
\text { induced } \\
\text { effects on } \\
\text { fatigue, and } \\
\text { on } C-F\end{array}$ & $\mathrm{p}$ & $\mathrm{N}$ & & & & & & & \\
\hline $\begin{array}{l}\text { xxi. Local vs. } \\
\text { non-local } \\
\text { damage }\end{array}$ & $\mathrm{S}$ & $\mathrm{Y}$ & & $\mathrm{N}$ & & 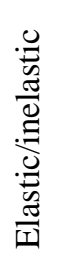 & & $\begin{array}{l}\text { Rb3251.11- } \\
\text { 2/Rb3252.11/ } \\
\text { Rb3252.31/ } \\
\text { RB3261.11// } \\
\text { RB3261.13/RB3262 }\end{array}$ & $\begin{array}{l}\text { RB3251.11-2 - limit of local primary stress/elastic analysis/ } \\
\text { Rb3252.11creep usage fraction locally/ RB3252.31 - Creep Rupture Usage fraction } \\
\text { locally / RB3261.111: defines maximum stress for S type damage using local } \\
\text { stress/ RB3261.13 : Fatigue at geometric discontinuities (zero radius). } \\
\text { RB3262 local stress used for determining significant creep damage. }\end{array}$ \\
\hline
\end{tabular}


Table 9.2.4.4-5 RCC-MR: Design Criteria

\begin{tabular}{|c|c|c|c|c|c|c|c|c|c|}
\hline 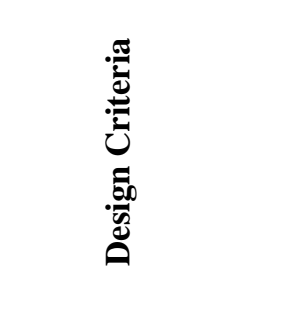 & 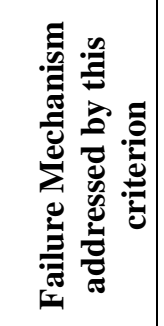 & 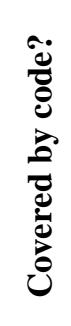 & 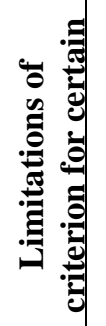 & 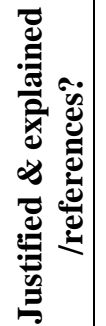 & 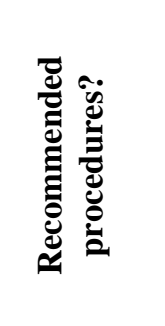 & 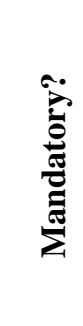 & 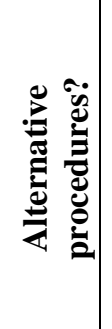 & 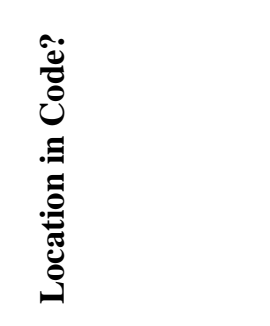 & 总 \\
\hline $\begin{array}{l}\text { xxii. Thermal } \\
\text { aging effects } \\
\text { on creep, } \\
\text { fatigue, limit } \\
\text { load }\end{array}$ & $\mathrm{t}$ & $\mathrm{Y}$ & & $\mathrm{Y}$ & $\begin{array}{l}\text { Elastic/ } \\
\text { Inelastic } \\
\text { for stress } \\
\text { evaluation }\end{array}$ & $\mathrm{N}$ & & RB3252 & $\begin{array}{l}\text { For significant creep: uses aging factor tabulated in A3.51 } \\
\text { for materials affected by thermal aging, applied to } \\
\text { allowable stress Sm. Temperature and time conditions } \\
\text { likely to produce a significant degradation are given in } \\
\text { A3.31 }\end{array}$ \\
\hline $\begin{array}{l}\text { xxiii. Elastic } \\
\text { Follow-up }\end{array}$ & $\begin{array}{l}\text { d, h, I, k, } \\
\quad \text { l, r, s }\end{array}$ & $\mathrm{Y}$ & & $\mathrm{N}$ & & & & $\begin{array}{l}\text { RB3261.1112.16 } \\
\text { /RB3262.123// } \\
\text { RB3262.2 }\end{array}$ & $\begin{array}{l}\text { Overstress for short duration. Elastic follow-up factor of } 3 \\
\text { for computing stress/ Elastic follow-up factor for fatigue } \\
\text { usage fraction-factor amplifies strain range// Elast-visco- } \\
\text { plast analysis automatically considers elastic followup }\end{array}$ \\
\hline $\begin{array}{l}\text { xxiv. Multi axial } \\
\text { effects on } \\
\text { creep, } \\
\text { fatigue and } \\
\text { C-F }\end{array}$ & r & $\begin{array}{l}\mathrm{Y} / \\
\mathrm{N}\end{array}$ & & & & & & & RB3226.1: $\overline{\sigma_{j}}$ can be replaced by $0.867 \overline{\sigma_{j}}+0.133 \operatorname{tr}_{j}$ \\
\hline
\end{tabular}




\subsubsection{ASME NH}

ASME NH is the U.S. Nuclear ETD Code, and is mandatory for design and construction of Class 1 nuclear components. Note, $\mathrm{NH}$ has not been officially recognized by the U.S. Nuclear Regulatory Commission to date, meaning it has neither been rejected nor accepted as the official U.S. Nuclear ETD Code due to the lack of construction of nuclear power plants in the U.S. since about 1980 . However, practically speaking, $\mathrm{NH}$ is viewed and accepted Internationally as the U.S. Nuclear ETD Code. The design methods and criteria contained within NH mainly address Primary Load Limits. Appendix T of NH addresses Deformation Controlled Limits; Appendix T is non-mandatory, and is recommended to address various failure mechanisms. Alternatively, one may specify other approaches in the Design Specification - assuming ample justification is made for such approaches.

The ASME elevated temperature design Code has a long history, and was the first international elevated temperature design Code to provide rules for construction and design which account for the effects of deformation and damage due to creep. A chronological map of its history is provided in Figure 9.2.5.1. In summary, significant updates, revisions, and additions to the Code occurred in large part due to U.S. developments of the Fast Flux Test Facility (FFTF). Afterwards, following response to the 3-Mile Island accident in the U.S., there was relatively limited activity and subsequent revisions to ASME NH. Meanwhile, International R\&D in ETD continued as indicated in Figure 9.2.5.2. U.S. nuclear energy programs continued to inject interest in ETD, but were limited in scope, duration, and funding relative to those prior to the 3-mile island accident. Several reviews were conducted for the U.S. Nuclear Regulatory Commission as indicated in Figures 9.2.5.1 and Figures 9.2.5.2; these reviews were to assess the status and need of ETD, and were not conducted to officially accept or reject NH for use in Nuclear ETD. 


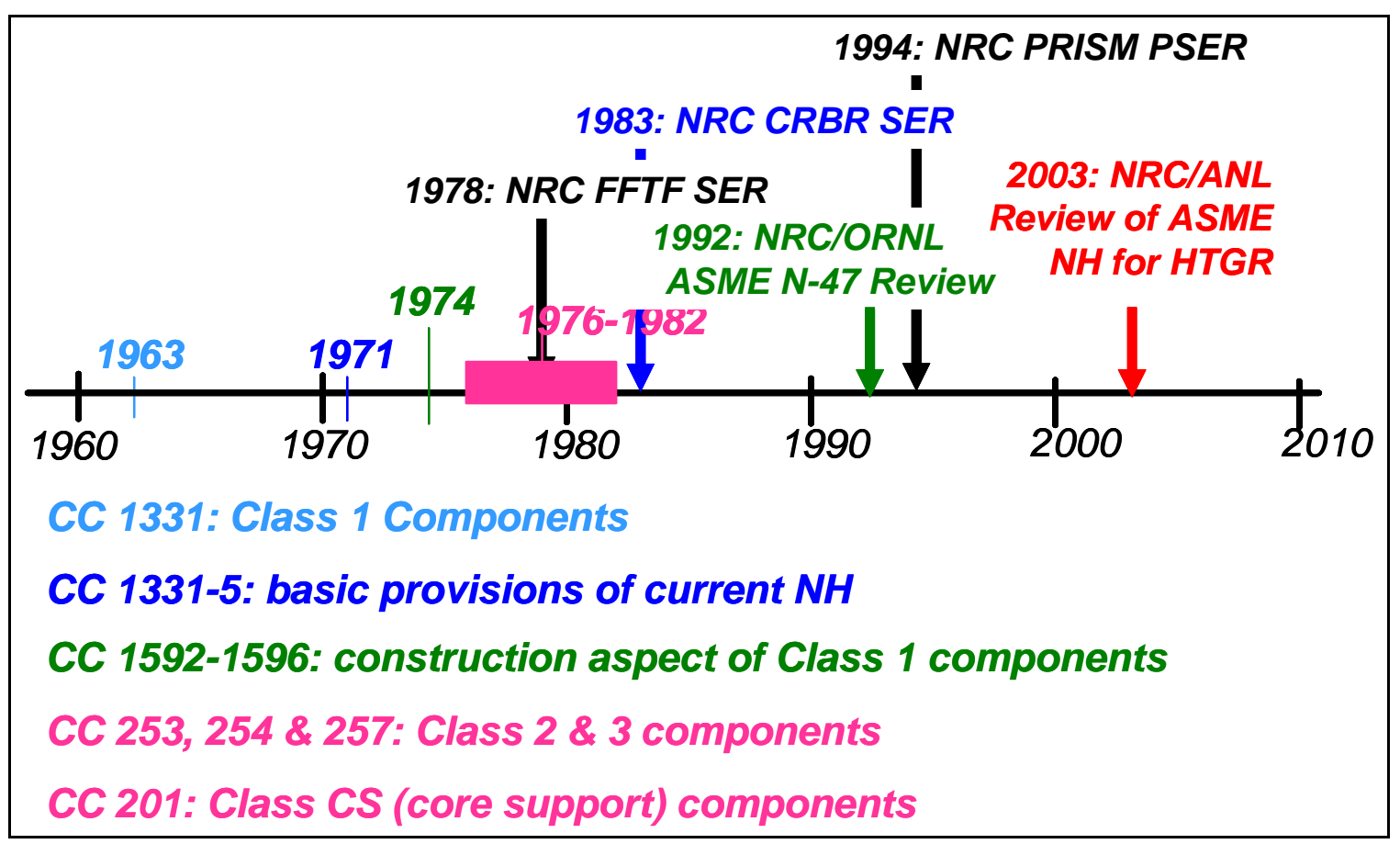

Figure 9.2.5.1 Timeline of ASME NH development

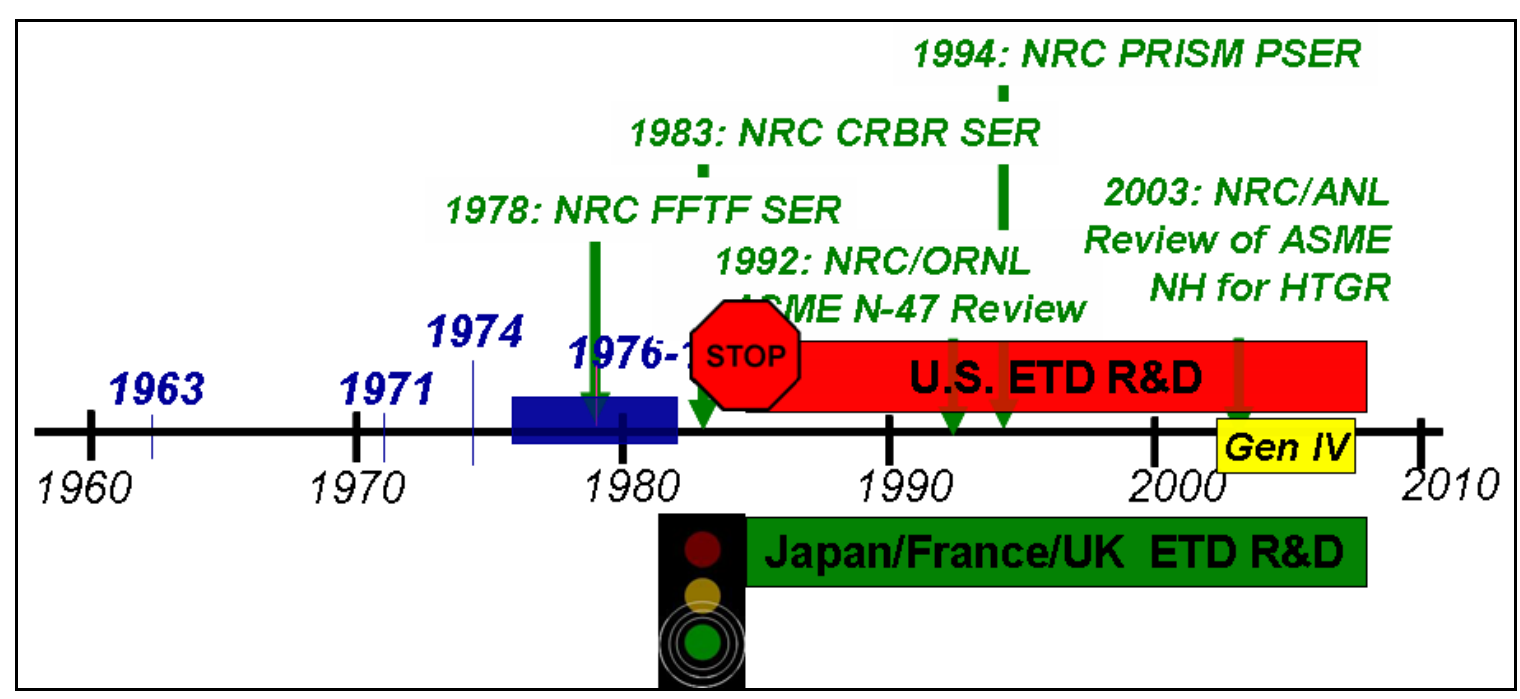

Figure 9.2.5.2 Post 1980 U.S. ETD R\&D limited relative to that internationally

\subsubsection{ASME NH - High Temperature}

In the Ideal ETD Code, a High Temperature Limit and a Criterion for Insignificant Creep were identified as important aspects for ETD Codes. Table 9.2.5.1 below summarizes this information for ASME NH. A time and temperature dependent stress allowable, $\mathrm{S}_{\mathrm{mt}}$, 
is defined for permissible materials. ASME NH has adopted standard criteria to distinguish between conditions where ETD (and ASME NH) is required, and when it is not. For ferritics, temperatures above $371^{\circ} \mathrm{C}$ require creep considerations, and the criterion is $427^{\circ} \mathrm{C}$ for austenitics. Currently, ASME NH does not permit use of nickel base super alloys, although a Draft Code Case for Alloy 617 exists. The only exception is that 718 is approved for use as a bolt material.

The definition for insignificant creep in ASME NH-3211 are vague, apparently purposefully so. The Code states "If the designer has demonstrated that the elevated temperature service parameters (time, stress, and temperature) do not introduce significant creep effects, then the experimental and analytical methods of Subsection NB shall be applicable." A footnote is referenced indicating that "A report documenting the experimental data or calculations based on experimental data or both shall demonstrate that the elevated temperature service does not introduce creep effects. This document shall be incorporated into the Stress Report (NCA-3550) and shall be approved by the Owner by means of a certified revision to the Design Specifications (NCA-3250).” Appendix T, T-1324, contains a more defined criterion based upon a use fraction rule on time to creep rupture of 0.1 or less and a cumulative creep strain limit of $0.2 \%$. Code Case N-201 Appendix XIX uses the identical Time-Temperature Limits as T-1324 and provides a figure (Figure XIX-1) indicating permissible time vs metal temperature.

\subsubsection{ASME NH - Design Loads}

The current status of ASME NH and how "Design Loads" are addressed relative to the Ideal ETD Code is summarized in Tables 9.2.5.2-1 and 9.2.5.2-2. Significant areas of interest are:

- Very limited guidance is provided for development of a load histogram and cycle definitions; Appendix T is not very clear regarding definition of load cycles for various simplified methods, e.g. A-Tests.

- The fundamental use and understanding of primary and secondary stresses, membrane, local, bending, and peak stresses are required.

- Primary loads are differentiation between various load categories, e.g. Service Level A \& B, C, and D, but is very general.

- Cyclic structural loading is limited to elastic regimes for some simplified approaches in Appendix T; shakedown for T-1324; elastic, shakedown, and plasticity for T-1332, and permits loading in the ratcheting regime in T-1333.

- A flow chart is provided to assist in understanding the requirements of NH, Table $\mathrm{NH}-3221-1$.

- Relatively recent revisions have permitted use of either Tresca or Mises yield criteria. 


\subsubsection{ASME NH - Failure Mechanisms}

ASME NH and Appendix T are organized, but significant opportunity exists to improve upon how information is presented, and perhaps a revision of the organizational approach. For instance, information related to satisfying a particular failure mechanism is scattered throughout the Code. Furthermore, ASME NH has no references, other than various additional sections within the Code itself. The authors' believe that reference to literature and other reports that support the concepts for which the Code is based would be very valuable and useful, particularly for justification to the NRC of either Code rules or similar approaches but modifications of Code approaches that may be utilized in the Stress Report or Design Specifications.

Tables 9.2.5.3-1 and 9.2.5.3-2 summarize the Failure Mechanisms of the Ideal ETD Code and how ASME NH and Appendix T address them. Highlights of the comparison are as follows:

- Clarity may be required between failure mechanisms of excessive deflection vs. excessive deformation.

- Strain limits are 1, 2, and 5\% for average through wall, average plus bending at a surface, and peak strains.

- Nonductile fracture is addressed by limitation of materials and service conditions.

- Fatigue is addressed in terms of nucleation of an engineering size crack, e.g. 5 $\mathrm{mm}$.

- Mass transfer, corrosion effects, excessive deformation under steady creep loading impacting functionality, cyclic creep softening (deformation and rupture), and irradiation effects are addressed by a general disclaimer.

- Creep buckling rules are limited to simple structures and loading conditions.

- Weldments are recognized to include metallurgical notches, and permissible strains are reduced by a factor of 2 relative to parent material.

- Thermal aging effects are only considered relative to short term strength effects, e.g. UTS and YS.

- Elastic follow-up is addressed by use of tables to assist in stress classification in Primary Load Limits section. Thermal membrane stresses are conservatively treated as primary for ratcheting analysis in Deformation Controlled Limits.

\subsubsection{ASME NH - Design Criteria / Procedures}

Table 9.2.5.4-1 through Table 9.2.5.4-6 summarize how various failure mechanisms are addressed through use of design criteria and/or procedures. Note, the procedures located in Appendix T may be viewed as mandatory, although alternative procedures are 
permissible provided they are justified in the Design Specification. For simplicity, Appendix $\mathrm{T}$ procedures are indicated as mandatory in these Tables.

Key points to mention are:

- NH does not permit inelastic analysis to satisfy Primary Load Limits, other than Service Level D loading; elastic analysis is only permitted.

- A means to account for redistribution of elastically calculated primary bending stresses due to creep is permissible.

- Limitation on the local primary membrane plus primary bending stress, with or without redistribution of stresses due to creep, effectively constitute a local primary stress failure criterion, as opposed to one where the primary stress of the 'core' addresses overall failure of the structure.

- The concept of an 'elastic core stress' is utilized to ensure that average strains through the wall do not exceed $1 \%$.

- A range of simplified design methods address cyclic loading in the elastic, shakedown, plasticity, and/or ratcheting regimes. Various degrees of conservatism, or non-conservatism, exist in these methods.

- Conventional C-F interaction diagram with a bi-linear damage rule is utilized.

- Strain concentration in weldments is addressed with a reduction in permissible strain by a factor of 2 .

- Buckling rules are based upon simplified structures and loading.

- No means to address the effects of corrosion, irradiation, and mass transport are provided.

- Strain limits are general and do not take into account the extent of creep ductility, or lack thereof, for a given material.

- Stress limits incorporate a strain limit, again without a lack of the extent of creep ductility, or lack thereof, for a given material. 
Table 9.2.5.1 ASME NH: High Temperature

\begin{tabular}{|c|c|c|c|c|}
\hline ID\# & Definition / Criterion & Covered? & Location & Comment \\
\hline $\mathrm{a}$ & High temperature limit & Yes & $\begin{array}{l}\text { NH-1120 \& App. I-14; NH } \\
3211 ; \\
\text { NB-3228.5(e) }\end{array}$ & $\begin{array}{l}\mathrm{S}_{\mathrm{mt}} \text { : creep significant at temperatures exceeding } \\
700 \mathrm{~F} \text { (371C) for ferritics or } 800 \mathrm{~F} \text { (427C) for } \\
\text { austenitics; or demonstrate insignficant }\end{array}$ \\
\hline $\mathrm{b}$ & $\begin{array}{l}\text { Criterion or definition of } \\
\text { temperature limit for } \\
\text { insignificant creep effects }\end{array}$ & Yes & $\begin{array}{l}\text { NH-3211(c); } \\
\text { App T-1324 }\end{array}$ & $\begin{array}{l}\text { Data and/or calculations in Stress Report \& } \\
\text { Design Specifications (NCA-3250); insignificant } \\
\text { creep criteria: time fraction rule, creep strain } \\
\text { limit, shakedown limit }\end{array}$ \\
\hline
\end{tabular}


Table 9.2.5.2-1 ASME NH: Design Loads

\begin{tabular}{|c|l|c|l|l|}
\hline ID\# & \multicolumn{1}{|c|}{ Definition / Criterion } & Covered? & \multicolumn{1}{|c|}{ Location } & \multicolumn{1}{c|}{ Comment } \\
\hline a & $\begin{array}{l}\text { Guidelines for load historgram \& } \\
\text { cycle definition }\end{array}$ & $\begin{array}{c}\text { General } \\
\text { Disclaimer }\end{array}$ & $\begin{array}{l}\text { NH-3112, NH-3114, NH- } \\
\text { 3213.15, NH-3213.16; } \\
\text { T-1321,T-1325 \& NB-3650 / } \\
\text { NB-3222. }\end{array}$ & $\begin{array}{l}\text { Very limited, out of scope; } \\
\text { Design Specification (NCA-3250), } \\
\text { restrictions on QR due to residual stresses or } \\
\text { carry-over' stresses. }\end{array}$ \\
\hline b & Glossary of terms & Yes & NH-3213 & $\begin{array}{l}\text { Some don't apply to NH, e.g. 3213.25 } \\
\text { Plastic Analysis - Collapse Load. }\end{array}$ \\
\hline c & Definition of stress intensities & Yes & NH-3215 & \\
\hline e & $\begin{array}{l}\text { Definition of strain intensities } \\
\text { Definition of primary vs secondary } \\
\text { stresses }\end{array}$ & Yes & $\begin{array}{l}\text { NH-3213.8 \& NH-3213.9; } \\
\text { NH-3217 }\end{array}$ & $\begin{array}{l}\text { Glossary terms only; } \\
\text { Table NH-3217 (tabulated 'rules'); }\end{array}$ \\
\hline f & $\begin{array}{l}\text { Definition of membrane, bending, } \\
\text { peak stresses }\end{array}$ & Yes & $\begin{array}{l}\text { NH-3213.6, NH-3213.7, NH- } \\
3213.11\end{array}$ & Presume basis would be same as for stress. \\
\hline g & Procedure for stress linearization & No & NH-3213, NH-3217 & Glossary terms \\
\hline
\end{tabular}


Table 9.2.5.2-2 ASME NH: Design Loads

\begin{tabular}{|c|c|c|c|c|}
\hline ID\# & Definition / Criterion & Covered? & Location & Comment \\
\hline \multirow[t]{7}{*}{$\mathrm{h}$} & Differentiated load catagories: & & & \\
\hline & design loads & Yes & NH-3112, NH-3113 & Very general \\
\hline & normal service loads & Yes & NH-3112, NH-3113 & Very general \\
\hline & frequent abnormal loads & Yes & NH-3112, NH-3113 & Very general \\
\hline & infrequent abnormal loads & Yes & NH-3112, NH-3113 & Very general \\
\hline & limiting fault loads & Yes & NH-3112, NH-3113 & Very general \\
\hline & test loads & Yes & NH-3112, NH-3113 & Very general \\
\hline $\mathrm{i}$ & $\begin{array}{l}\text { Flow chart for design by analysis or } \\
\text { rule/procedure }\end{array}$ & Yes & NH-3221 & Table NH-3221-1; addresses both NH and App. T \\
\hline $\mathrm{j}$ & $\begin{array}{l}\text { Yield function requirements (e.g. Tresca vs } \\
\text { Mises) }\end{array}$ & Yes & NH-3212 & Elastic anlaysis: Tresca. Inelastic analysis (Mises, open) \\
\hline
\end{tabular}


Table 9.2.5.3-1 ASME NH: Failure Mechanisms

\begin{tabular}{|c|c|c|c|c|}
\hline ID\# & Definition / Criterion & Covered? & Location & Comment \\
\hline a & $\begin{array}{l}\text { Limit load collapse, single load } \\
\text { application }\end{array}$ & Yes & $\begin{array}{l}\text { NH-3221, NH-3222.1(b); } \\
\text { NH-3213.xx }\end{array}$ & $\begin{array}{l}\text { Sm,So in App I-14; } \\
\text { Glossary exists, but it only supports general inelastic } \\
\text { analysis (no simplified analysis) }\end{array}$ \\
\hline $\mathrm{b}$ & $\begin{array}{l}\text { Excessive deformation limiting } \\
\text { functionality, under a single load } \\
\text { application }\end{array}$ & $\begin{array}{l}\text { General } \\
\text { Disclaimer }\end{array}$ & NH-3111.1(a), T-1210 & $\begin{array}{l}\text { Deformation is mentioned; may need both deformation and } \\
\text { displacement, but not mentioned. }\end{array}$ \\
\hline c & $\begin{array}{l}\text { Structural instability or buckling, under } \\
\text { a single load application }\end{array}$ & Yes & $\begin{array}{l}\text { NH-3222.1(c), NB-3133, NH-3251, } \\
\text { NH-3252, T-1510(e), T-1521 }\end{array}$ & Must consider defects/tolerances (initial or service induced) \\
\hline $\mathrm{d}$ & $\begin{array}{l}\text { Progressive collapse by ratcheting } \\
\text { under cyclic load }\end{array}$ & Yes & $\mathrm{T}-1210 ; \mathrm{T}-1300, \mathrm{~T}-1310$ & Strain limits \\
\hline e & Nonductile fracture & Yes & NH-3241 & $\begin{array}{l}\text { Materials limited to ensure ductile fracture; must justify in } \\
\text { Stress Report (NCA-3250) also. }\end{array}$ \\
\hline $\mathrm{f}$ & $\begin{array}{l}\text { Fatigue failure (nucleation on order of } \\
5 \mathrm{~mm})\end{array}$ & Yes & T-1400; NB-3000 & Tables T-1420-1X \\
\hline g & $\begin{array}{l}\text { Collapse or breach of pressure } \\
\text { boundary due to corrosion, mass } \\
\text { transfer phenomenon, etc. }\end{array}$ & $\begin{array}{l}\text { General } \\
\text { Disclaimer }\end{array}$ & NH-2160 & Responsibility of owner, Design Specification (NCA-3250) \\
\hline h & $\begin{array}{l}\text { Excessive deformation leading to loss } \\
\text { of functionality, due to creep under } \\
\text { steady load }\end{array}$ & $\begin{array}{l}\text { General } \\
\text { Disclaimer }\end{array}$ & NH-3250, T-1210 & Design Specification (NCA-3250) \\
\hline $\mathrm{i}$ & Creep rupture & Yes & $\begin{array}{l}\text { NH-3221, NH-3222.1(b); } \\
\text { NH-3213.xx }\end{array}$ & $S_{t}($ App I-14) \\
\hline
\end{tabular}


Table 9.2.5.3-2 ASME NH: Failure Mechanisms

\begin{tabular}{|c|c|c|c|c|}
\hline ID\# & Definition / Criterion & Covered? & Location & Comment \\
\hline $\mathrm{j}$ & Creep buckling & Yes & $\mathrm{T}-1500, \mathrm{~T}-1520$ & Limited applicability to simple components, without defects \\
\hline $\mathrm{k}$ & Enhanced creep & Yes & T-1220; T-1310; T-1324 & load control 1\%; strain limits 1\%,2\%,5\%; insignificant creep \\
\hline l & Creep-Fatigue & Yes & $\begin{array}{l}\text { T-1324; Figs T-1420-1X; Fig T- } \\
\text { 1420-2; T-1432(g); Fig T-1800; } \\
\text { Figs I-14.6 }\end{array}$ & $\begin{array}{l}\text { insignificant creep; strain-life, C-F interaction, creep strain, } \\
\text { isochronous curves, rupture curves }\end{array}$ \\
\hline $\mathrm{m}$ & Accelerated creep rupture & No & \multicolumn{2}{|c|}{ Weakly covered by C-F, but would apply locally } \\
\hline $\mathrm{n}$ & $\begin{array}{l}\text { Softening enhanced cyclic creep } \\
\text { rupture }\end{array}$ & $\begin{array}{c}\text { General } \\
\text { Disclaimer }\end{array}$ & NH-3214.2 & If inelastic analysis is being used. \\
\hline o & $\begin{array}{l}\text { Softening enhanced cyclic creep } \\
\text { deformation }\end{array}$ & $\begin{array}{c}\text { General } \\
\text { Disclaimer }\end{array}$ & NH-3214.2 & If inelastic analysis is being used. \\
\hline $\mathrm{p}$ & Irradiation effects & $\begin{array}{c}\text { General } \\
\text { Disclaimer }\end{array}$ & NH-110(e) & $\begin{array}{l}\text { e.g. swelling, creep, plastic flow localization, embrittlement, } \\
\text { reduction of fatigue, C-F, etc. }\end{array}$ \\
\hline $\mathrm{q}$ & Weldments (strength, strain) & & $\mathrm{T}-1715(\mathrm{C}-\mathrm{F})$ & \\
\hline $\mathrm{r}$ & $\begin{array}{l}\text { Multiaxial effects on creep, fatigue, C- } \\
\text { F }\end{array}$ & & Figures T-1432-2, T-1432-3 & \\
\hline S & Local vs. distributed damage & Indirectly & \multicolumn{2}{|c|}{$\begin{array}{l}\text { Code has limited materials approved for service (time \& temperature); intent is to limit to creep ductile } \\
\text { materials (e.g. } \lambda>5 \text { ) }\end{array}$} \\
\hline $\mathrm{t}$ & Thermal aging & Mixed & NH-3225-2 & $\begin{array}{l}\text { Only reduction in YS, UTS; no consideration on creep or fatigue } \\
\text { damage, or creep deformation rates }\end{array}$ \\
\hline $\mathrm{u}$ & Elastic follow-up & Yes & $\begin{array}{l}\text { NH-3138; T-1331(d); T-1434; T- } \\
1510\end{array}$ & $\begin{array}{l}\text { General consideration; strain limits, strain range in piping, } \\
\text { buckling. }\end{array}$ \\
\hline
\end{tabular}


Table 9.2.5.4-1 ASME NH: Design Criteria / Procedures

\begin{tabular}{|c|c|c|c|c|c|c|c|c|c|}
\hline 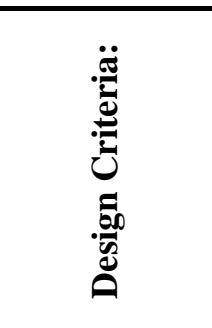 & 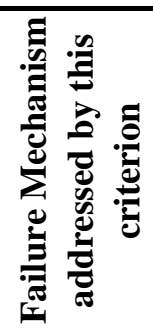 & 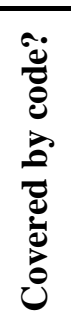 & 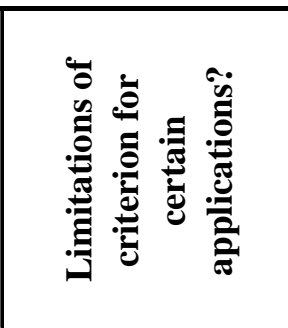 & め & 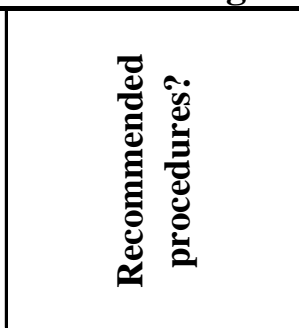 & 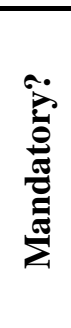 & 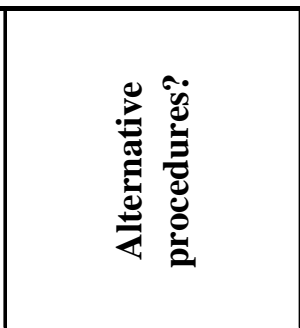 & 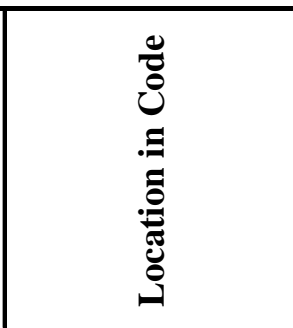 & 芑 \\
\hline i. Limit Load & $\mathrm{a}$ & Yes & $\begin{array}{l}\text { Clearly states elastic } \\
\text { analysis only, unless } \\
\text { Level D loading } \\
\text { (NH3223-3224); NH- } \\
\text { 3212(b) unclear if } \\
\text { inelastic analysis is } \\
\text { permitted in general. } \\
\text { NH3214.2 eludes to } \\
\text { inelastic analysis, but } \\
\text { generally for } \\
\text { Appendix T only. }\end{array}$ & No. & Elastic rules & Yes. & $\begin{array}{l}\text { All based upon elastic } \\
\text { analysis, except Level } \\
\text { D limits permits } \\
\text { inelastic. } \\
\text { Appendix T may } \\
\text { indirectly permit use } \\
\text { of inelastic analysis; } \\
\text { limit load is special } \\
\text { subset or case of cyclic } \\
\text { loading (e.g. Q=0, } \\
\text { Pm<Sy to avoid } \\
\text { ratchet is a limit load } \\
\text { criterion). }\end{array}$ & $\begin{array}{l}\text { NH-1110(d) } \\
\text { NH-3222.1(a) Eqn(1), } \\
\text { Eqn(2), NB-3133, } \\
\text { NH3252; } \\
\text { NH-3223(c) Eqn(4); } \\
\text { NH-3224(a) Eqn(7), } \\
\text { (c) Eqn(9); } \\
\text { NH-3225 (b) Eqn(12), } \\
\text { NH-3649.2;NH- } \\
3651(a)\end{array}$ & $\begin{array}{l}\text { General comment in } \\
\text { Intro; } \\
\text { Design limits; } \\
\text { Level A \& B limits; } \\
\text { Level C limits; } \\
\text { Level D limits; } \\
\text { Experimental Analysis } \\
\text { of Piping, Analysis. }\end{array}$ \\
\hline $\begin{array}{l}\text { ii. Excessive } \\
\text { deflection }\end{array}$ & $\mathrm{b}$ & $\mathrm{Y} / \mathrm{N}$ & $\begin{array}{l}\text { Only useful if default } \\
\text { strain limits satisfy } \\
\text { deflection limitations. }\end{array}$ & No. & $\begin{array}{l}\text { Must satisfy in Design } \\
\text { Specification (NCA- } \\
\text { 3250). Manufacturer } \\
\text { supply criteria to } \\
\text { Owner. }\end{array}$ & Yes. & $\begin{array}{l}\text { Appendix T - A\&B } \\
\text { Test: } \\
\text { T-1310, T-1322,T- } \\
\text { 1323,T-1324, T-1331, } \\
\text { T-1332, T-1333 }\end{array}$ & NH-3252 & $\begin{array}{l}\text { NH limits deformation } \\
\text { indirectly via Smt } \\
\text { stress allowable; App } \\
\text { T limits deformation } \\
\text { indirectly via strain } \\
\text { limits. } \\
\text { Strain does not equate } \\
\text { directly to deflection. }\end{array}$ \\
\hline
\end{tabular}


Table 9.2.5.4-2 ASME NH: Design Criteria / Procedures

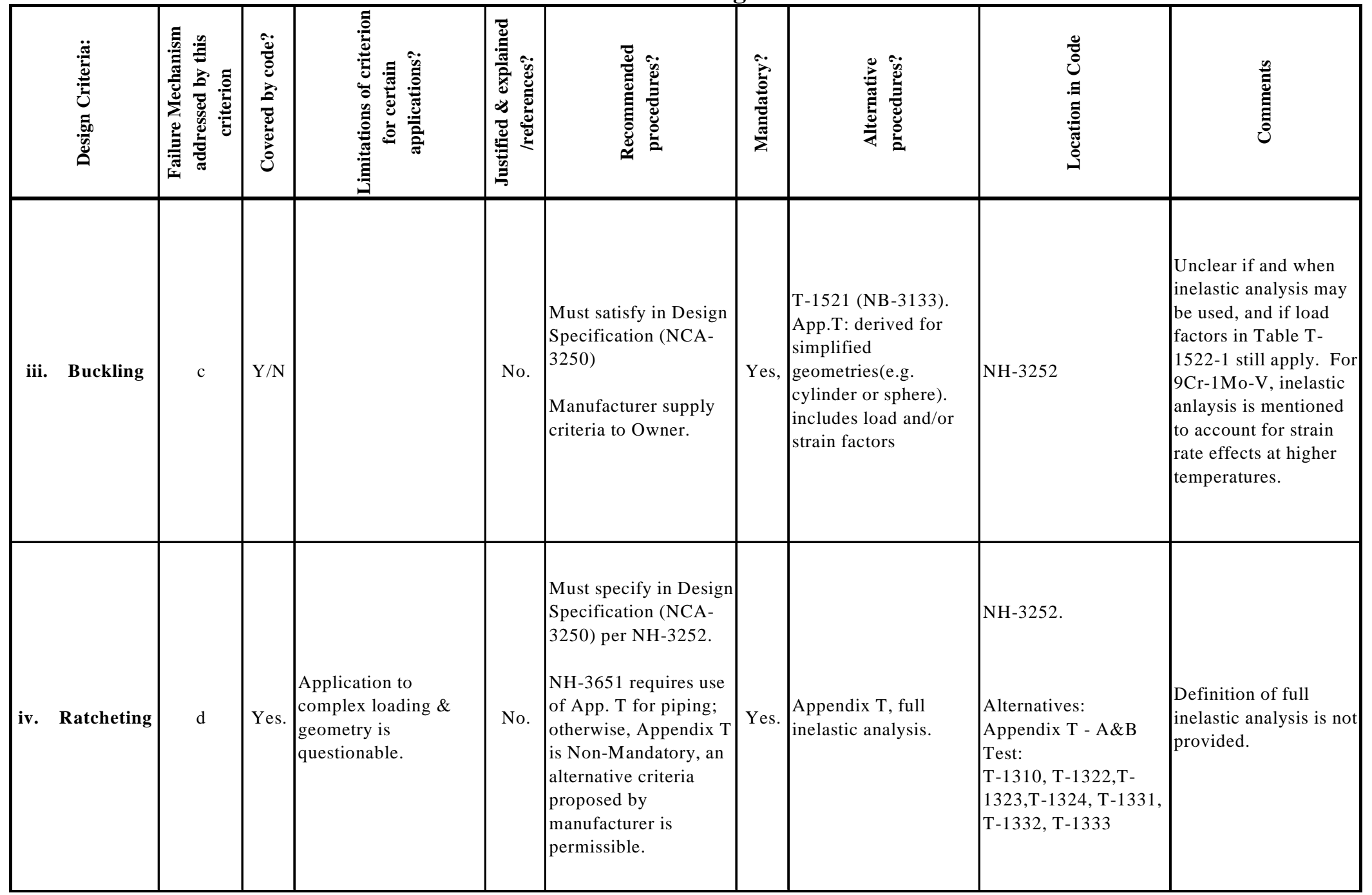


Table 9.2.5.4-3 ASME NH: Design Criteria / Procedures

\begin{tabular}{|c|c|c|c|c|c|c|c|c|c|}
\hline 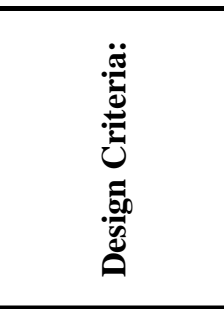 & 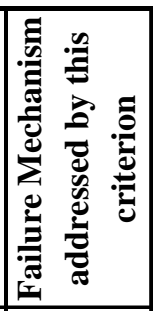 & 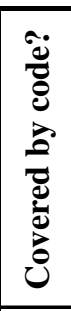 & 苛 & 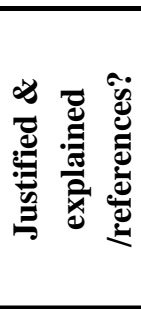 & 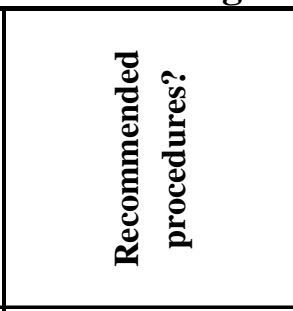 & 总 & 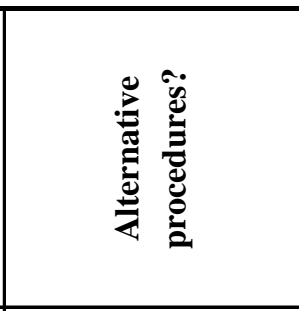 & 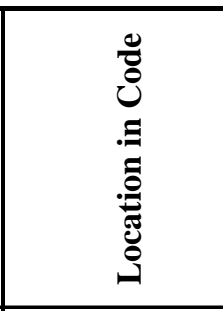 & ن气 \\
\hline v. Fracture & e & $\mathrm{Y} / \mathrm{N}$ & $\begin{array}{l}\text { Material dependent; no } \\
\text { mention of section } \\
\text { size, strain rate, grain } \\
\text { size, etc. }\end{array}$ & No. & $\begin{array}{l}\text { Stress Report (NCA- } \\
3550 \text { ); } \\
\text { Appendix G for } \\
\text { insignificant creep in } \\
\text { ferritics. Not required } \\
\text { for 304SS, 316SS, } \\
800 \mathrm{H}\end{array}$ & Yes. & $\begin{array}{l}\text { Appendix G of Section } \\
\text { III for ferritics if } \\
\text { insignificant creep. }\end{array}$ & NH-32414 & $\begin{array}{l}\text { 304SS, } 316 \mathrm{SS}, 800 \mathrm{H} \\
\text { must be reconsidered } \\
\text { if fabrication alters } \\
\text { fracture mode to } \\
\text { brittle. }\end{array}$ \\
\hline vi. Fatigue & f & Yes. & & No. & $\begin{array}{l}\text { Design Specification } \\
\text { (NCA-3250); } \\
\text { Manufacturer supply } \\
\text { criteria to Owner. }\end{array}$ & Yes. & $\begin{array}{l}\text { Appendix T (T-1400) } \\
\text { may be used to satisfy. }\end{array}$ & NH-3252 & $\begin{array}{l}\text { Fatigue addressed in } \\
\text { creep-fatigue criteria; } \\
\text { if insignificant creep. } \\
\text { Criteria covered under } \\
\text { C-F within this table. }\end{array}$ \\
\hline vii. Corrosion & g & No. & & & & & & & General disclaimer. \\
\hline $\begin{array}{l}\text { viii. Excessive } \\
\text { creep } \\
\text { deformation }\end{array}$ & $\mathrm{h}$ & Yes. & $\begin{array}{l}\text { Generalized criteria - } \\
\text { not specific to extent } \\
\text { of creep ductility or } \\
\text { lack thereof. }\end{array}$ & No. & $\begin{array}{l}\text { Test A \& B } \\
\text { Strain Limiting Stress, } \\
\text { St }\end{array}$ & Yes. & Inelastic analysis & T1300/ NH3000 & $\begin{array}{l}\text { Strain is limited to } \\
1 \%, 2 \% \text { \& } 5 \% \text {, AND } \\
\text { stress intensity is } \\
\text { dictated by limiting } \\
\text { strain to } 1 \%\end{array}$ \\
\hline
\end{tabular}


Table 9.2.5.4-4 ASME NH: Design Criteria / Procedures

\begin{tabular}{|c|c|c|c|c|c|c|c|c|c|}
\hline 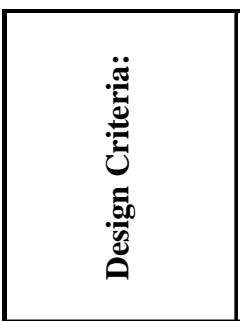 & 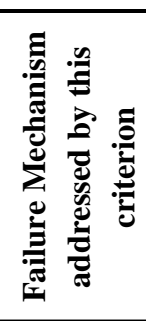 & 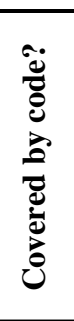 & 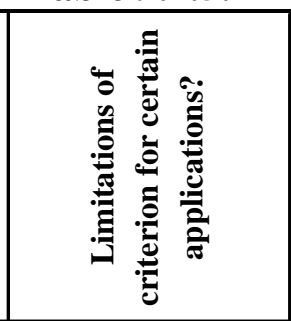 & 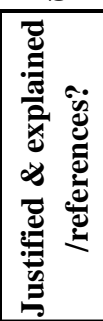 & 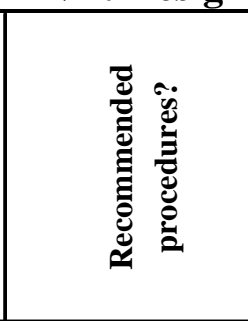 & 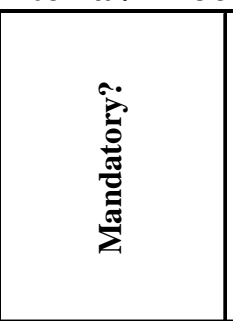 & 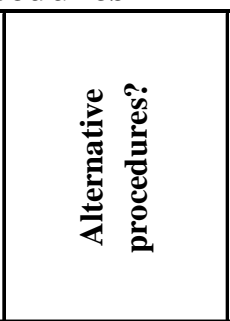 & 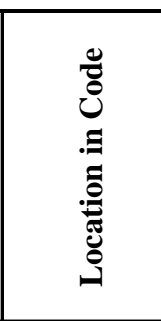 & 泀 \\
\hline $\begin{array}{l}\text { ix. Creep } \\
\text { buckling }\end{array}$ & h,b & Yes. & Limited to press vessel & No. & Apply load factors & Yes. Load factors & & T1521-1522 & \\
\hline x. $\quad$ Creep rupture & b,h,i & Yes. & & No. & Damage Rule & Yes. & & T-1400 & \\
\hline$\left|\begin{array}{cc}\text { xi. } & \text { Enhanced } \\
\text { Creep } & \text { Deformation }\end{array}\right|$ & $\mathrm{j}$ & Yes. & $\begin{array}{l}\text { A Tests are most } \\
\text { conservative; B Tests } \\
\text { are less conservative. }\end{array}$ & No. & Tests A \& B & Yes. & Inelastic analysis. & T-1300 & \\
\hline $\begin{array}{c}\text { xii. } \begin{array}{c}\text { Creep-Fatigue } \\
\text { Interaction }\end{array} \\
\text { ant }\end{array}$ & $\mathrm{k}$ & Yes. & $\begin{array}{l}\text { Linear damage rule } \\
\text { limitations. }\end{array}$ & No. & $\begin{array}{l}\text { Time/cycle fraction } \\
\text { rule }\end{array}$ & Yes. & $\begin{array}{l}\text { Simplified } \\
\text { approach or full } \\
\text { inelastic analysis. }\end{array}$ & T-1400 & \\
\hline
\end{tabular}


Table 9.2.5.4-5 ASME NH: Design Criteria / Procedures

\begin{tabular}{|c|c|c|c|c|c|c|c|c|c|}
\hline 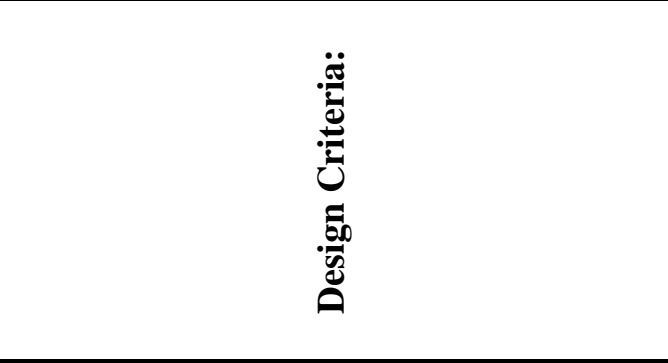 & 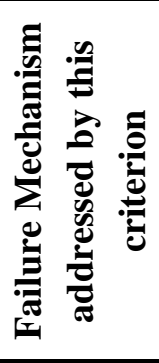 & 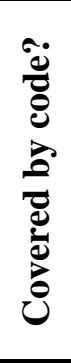 & 苞 & 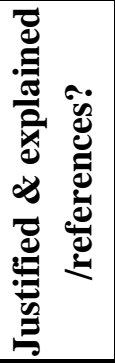 & 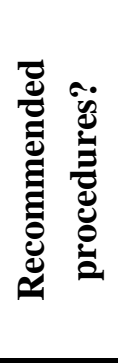 & 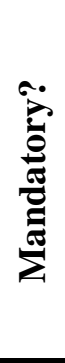 & 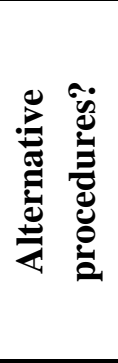 & 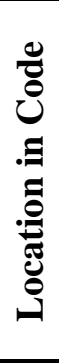 & نُّ \\
\hline xiii. Accelerated Cyclic Creep Rupture & 1 & No. & & & & & & & \\
\hline $\begin{array}{c}\text { xiv. Softening Enhanced Cyc. Creep } \\
\text { Deformation } \\
\end{array}$ & $\mathrm{m}$ & No. & & & & & & & \\
\hline $\begin{array}{c}\text { xv. Softening Enhanced Cyc. Creep } \\
\text { Rupture } \\
\end{array}$ & $\mathrm{n}$ & No. & & & & & & & \\
\hline xvi. Irradiation induced swelling & o & No. & & & & & & & \\
\hline xvii. Irradiation induced creep & o & No. & & & & & & & \\
\hline $\begin{array}{l}\text { xviii. Plastic Flow localization due to } \\
\text { irradiation (dislocation tunneling) }\end{array}$ & o & No. & & & & & & & \\
\hline $\begin{array}{l}\text { xix. Ductility exhaustion due to irradiation } \\
\text { exposure }\end{array}$ & o & No. & & & & & & & \\
\hline $\begin{array}{l}\text { xx. Irradiation induced effects on fatigue, } \\
\text { and on C-F }\end{array}$ & o & No. & & & & & & & \\
\hline
\end{tabular}


Table 9.2.5.4-6 ASME NH: Design Criteria / Procedures

\begin{tabular}{|c|c|c|c|c|c|c|c|c|c|}
\hline 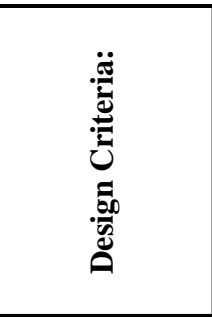 & 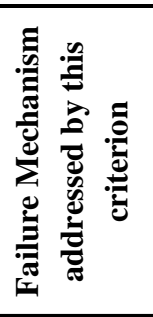 & 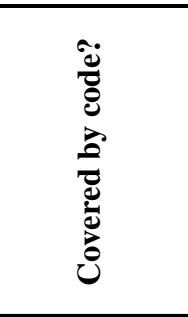 & 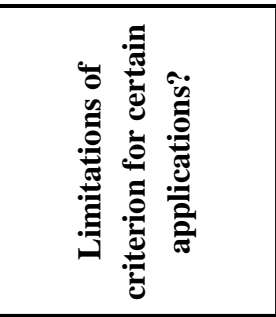 & 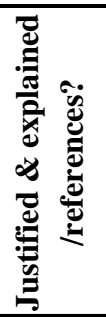 & 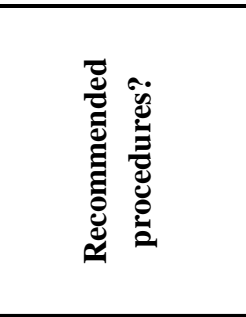 & 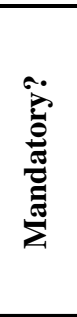 & 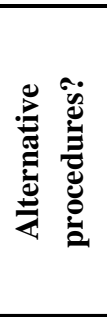 & 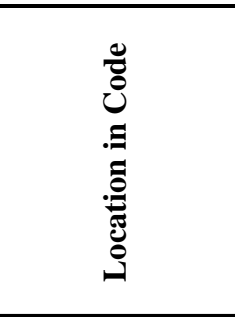 & نै \\
\hline $\begin{array}{l}\text { xxi. Local vs. } \\
\text { non-local } \\
\text { damage }\end{array}$ & $\mathrm{p}$ & Tim work on! & ? & ? & $\begin{array}{c}\text { Elastic, inelastic } \\
\text { analysis }\end{array}$ & ? & ? & $\mathrm{T}-1400$ & \\
\hline $\begin{array}{l}\text { xxii. Thermal } \\
\text { aging effects on } \\
\text { creep, fatigue, } \\
\text { limit load }\end{array}$ & $\mathrm{q}$ & $\mathrm{Y} / \mathrm{N}$ & ?? & No. & $\begin{array}{l}\text { Limit isoch curves } \\
\text { time/temp to avoid } \\
\text { aging }\end{array}$ & Yes. & & $\mathrm{T}-1400$ & \\
\hline $\begin{array}{l}\text { xxiii. Elastic } \\
\text { Follow-up }\end{array}$ & $\mathrm{r}$ & Yes. & $\begin{array}{l}\text { Thermal membrane } \\
\text { stress is conservativly } \\
\text { treated as Primary for } \\
\text { ratcheting rules. }\end{array}$ & No. & & Yes. & & $\begin{array}{c}\text { NH } 3138, T-1331 \\
\text { (d) }\end{array}$ & \\
\hline
\end{tabular}




\subsubsection{API579}

API 579 is not a design code. It is a guideline for the assessment of remaining life of plant in continuing operation. As such many of the assessment techniques presented in it are relevant to design with minor changes in objectives. This is particularly so because of the way it evolved.

About 15 years ago, both ASME and API came to realize the need for a regulated approach to assessment of existing plant with the object of determining remaining life after some period of operation. API approached the problem as an exercise in assembly of the best current methods of analysis for the wide range of damage mechanisms encountered in the petrochemical industry since, at the time, there was a tendency for fitness-for-service assessments to be carried out in a variety of ways, depending on the experience and resources of the analysts given the task. The list of mechanisms addressed was comprehensive from the outset, drawn as it was from ongoing plant experience, and the methods put forward to deal with them were drawn from the best available to the industry. Although FFS is not the same as design, the need for evaluation criteria is the same. Therefore, API579 contains practically all the elements required in a design code.

The parallel ASME effort was slower to develop so, in 2000, since the objectives of the two initiatives were similar, and in fact the participants were in many cases the same individuals, it was decided to merge the two into a single Joint ASME/API Task Force on FFS, with simultaneous standing as an API guideline, retaining the number 579, as well as an ASME Standard.

API579's origins have led to a different structure in places from that employed by the ASME Code. Since it is derived from an effort to produce a compendium of procedures for dealing with a range of damage mechanisms of direct concern to plant operators, it emphasizes individual types of defects, such as material losses due to corrosion, dents and pitting, as well as evaluation and remedial action in the event of cracklike defects being found. Some of these concerns are, currently, deemed to be beyond the scope of the conventional Sections of the ASM BPV Code.

Methods of defining analysis techniques also differ from Code practice to some extent. API 579 has been developed largely by participants who are either familiar with the ASME Code or are required to conform with it on a regular basis. In such situations as the definition of stress types (classification) or material assessment allowables, together with the simpler screening assessment methods, API 579 conforms closely with ASME Code practice. If there are differences, this lies in allowing more discretion on the part of the user to employ advanced methods of analysis, notably FEA, as a routine matter. Consequently, simplified methods holding a prominent position in Code procedures, such as the Bree Diagram for shakedown and ratcheting are absent, replaced by direct cyclic analysis of the actual component geometry. Therefore some of the design rules in the Code have no exact equivalents in API 579. 
A major difference between API 579 and current Code practice is a self contained and very comprehensive treatment of material failure mechanisms, including an extensive material database addressing material parameters, such as cyclic stress/strain curves and constitutive equations for creep. The database is not as extensive as Section II of the Code, to some extent because it does not call out material types according to their form (plate, tube, etc), but deals with the handful of materials most commonly used in plant operation. This database permits greater utilization of the detailed methods of structural analysis which have been developed in the past 20 years, based on the finite element technique and which, today, is a routine a tool for this purpose as was the slide-rule at one time.

API 579 is structured primarily as a guide for the assessment of components which have been discovered, in the field, to have suffered some form of damage. In order to speed the process of repair and return to operation, it is structured on 3 Levels. Level 1 is a simplified screening step which may be performed by plant operators and is therefore very conservative. Levels 2 and 3 are progressively more detailed levels of engineering analysis requiring the attention of a qualified engineer.

In keeping with its origins as a compendium of "best industrial practice”, API 579 allows the use of alternative procedures from other recognized codes, standards and guidelines where these are considered more appropriate. The same freedom extends to material data used in assessment. While a comprehensive range of material properties and associated evaluation methodologies are included in API 579, the user is permitted to user other sources if these own suitable credentials. In particular, R5 and R6 are specifically mentioned as permissible alternative FFS procedures.

API 579 is comprised of twelve (12) Parts : 1) Introduction, 2) FFS Engineering Assessment Procedure, 3) Brittle Fracture, 4/5/6) General metal loss through pitting, 7) Hydrogen damage, 8) Weld and Shell distortions, 9) Crack-like defects, 10) Components operating in the Creep Range, 11) Fire Damage, 12) Dents and Gouges, etc, 13)

Laminations, together with thirteen (13) Annexes. Parts 2 and 10, together with Annexes B.1. Stress Analysis overview, and C Material Properties, are relevant to this discussion.

\subsubsection{API579 - High Temperature}

Creep rupture, distortion and cracking under steady and cyclic loading are all addressed in Part 10 of API 579. High temperature operation is a major aspect of FFS, so that creep deformation and damages of all kinds hold a prominent place in the assessment procedures. However, since creep is an integral element of the component life cycle, no special methods are offered specifically to deal with creep as an isolated problem. Except for the simplest level of screening provided by a Level 1 assessment creep, together with all other material phenomena, are included in unified analyses which take all forms of deformation and damage into account as one. This means that API 579 does not offer special procedures for dealing with individual problems, such as ratcheting, enhanced 
creep, etc. These phenomena are identified as potential failure mechanisms but are addressed by a generic but detailed analysis of the load history which includes cyclic operation as well as hold periods at elevated temperature.

The only special attention given to creep as a unique problem is at Level 1, which seeks to exclude screen out the prospect of creep damage and avoid the complexities of carrying out a time dependent structural analysis if the temperature is too low for creep to be significant. This screening process is equivalent to the "negligible" creep criterion used in NH. However, the Level 1 procedure has no single answer but determines on a case-by-case basis, whether creep is likely to be a significant factor or not.

\subsubsection{API579 - Design Loads}

Table 9.2.6.2-1 and 9.2.6.2-2 summarize information relevant to 'Design Loads' for an Ideal ETD Code, including the Definition / Criterion, whether the Definition is 'Covered', the location in the Code where the Definition is addressed, and relevant comments. Of particular interest are the following:

The scope of API 579 includes all conceivable loading situations but, since it is in part, an after-the-fact evaluation of a prior load history, the categorization procedure differs in some respects from $\mathrm{NH}$.

The loadings employed in the original design of the component are used, where known, in preliminary phases of a FFS evaluation. Thereafter, actual loadings, both thermal and mechanical, are used for the assessment of prior damage and, in modified form, for the prediction of remaining life. The different levels of conservatism applied in Section III to "design" and Levels I through IV are still recognized and used in the assignment of different stress allowables to different combinations of pressure, gravity, external mechanical, wind and thermal loadings but, in place of grouping load cases under levels of severity, specific Safety margins are applied to a table of loading combinations. Significant points of interest in the use of API 579 are:

- Significant guidance is given for development of a load histogram and cycle definitions, as well as step-by-step guidelines for implementing them in an assessment.

- The fundamental concepts stress categorization, as laid out in Section III of the ASME Code, such as primary and secondary stresses, membrane, local, bending, and peak stresses are all drawn on as needed, depending on the Level selected for the assessment. For instance, simplified analysis based on linear elastic methods is one permissible route and API 579 provides detailed procedures for carrying out the associated stress linearization using several options, including one developed specifically for use in FEA of complex 3-dimensional geometries. utilized, although as will be discussed later, design criteria and procedures permit use of reference stress approaches which do not require stress linearization and categorization. API 579 also allows the use of detailed inelastic analysis which 
dispenses with stress classification on a section-by-section basis for the assessment of general structural failure mechanisms including gross load collapse, buckling, and ratcheting, with and without the inclusion of creep effects.

- Evaluation of imperfections is central to a FFS guideline. API 579 therefore gives considerable attention to preexisting defects and defects generated in service. In the past it has not been the policy of the ASME Code to address defects, either preexisting or generated in service, in what is stated to be a "construction code". In extending design practice to encompass finite life, as is inevitable in very high temperature applications for instance, that this policy may need to be modified, in which case the precedent given by API579 may become highly relevant.

\subsubsection{API579 - Failure Mechanisms}

The range of failure mechanisms addressed in API 579 is comprehensive as it applies to non-nuclear applications. It encompasses virtually all named mechanisms appearing in the ASME Code at present, many of them specifically and in detail, with the exception of damage caused by nuclear radiation.

Damage mechanisms involving metallurgical deterioration such as aging and cyclic softening are recognized in API 579 but these effects are dealt with at present by the extraction and testing, when possible, of specimens taken from components after time in service. One singular exception is the cyclic softening behavior of Grade 91 steel, which is known to influence the creep/fatigue strength of this material. For this reason, a specific methodology has been developed by MPC for this problem and is likely to be incorporated in the compendium of material descriptions contained in API 579.

Tables 9.2.6.3-1 thru 9.2.6.3-3 summarize the extent by which failure mechanisms of an Ideal ETD Code are addressed by the R5 procedure. The information is summarized in the same manner as Tables 9.2.6.2-1 and 9.2.6.2-2. Three possibilities exist for response to the column labeled 'Covered?', “Yes”, “No”, and "Yes/No”, with the latter requiring an explanation. "Yes/No" indicates that the Code does not explicitly address the failure mechanism or does in a limited way or circumstances; but the Code does address the mechanism indirectly to some extent. For example, 'Excessive deformation limiting functionality, under a single load application’ is indirectly covered by safety factors on Sy for limit loads.

\subsubsection{API579 - Design Criteria / Procedures}

Tables 9.2.6.4-1 through 9.2.6.4-7 summarize the various design criteria of an Ideal ETD Code and how API 579 addresses them or not. The first column indicates the design criteria, and the failure mechanism addressed by the criterion is listed in the second column; whether or not the API 579 covers the design criteria is indicated in the third 
column. Limitations of the criteria, justification \& explanation / references for the design criteria, and recommended procedures are also listed, including whether or not they are mandatory or not. Alternative procedures are summarized, including the locations in the Code that address the criteria. Finally, comments are included as appropriate.

API 579 accepts the use conventional stress linearization procedures when the assessment is carried by simplified elastic analysis. In this respect, the assessment procedures are essentially the same as those outlined in Sections III, and VIII/2 of the ASM Code.

Mechanical and thermal ratcheting are assessed initially by very simplified versions of the " $3 S \mathrm{Sm}$ " procedure for cyclic mechanical loads and rudimentary version of the Bree Diagram for cyclic thermal stress involving a linear or parabolic through-thickness temperature gradient. API 579 passes on quickly to description of a detailed structural evaluation, including an analysis of at least one instance of every cycle in the loading histogram, in which collapse, excessive deformation and progressive failure by ratcheting are assessed from FE results. This procedure encompasses creep deformations and therefore deals automatically with issues such as enhanced creep rupture and creep ratcheting. This approach is clearly more practical in the context of a FFS guideline, where the geometry and the nature of the load history are already known to some degree of certainty, than in a design code where geometries and loads are merely notional.

Failure by excessive creep deformation is not addressed as a separate problem by API 579 , since it is dealt with automatically as part of the more general cyclic load analysis recommended.

The failure criterion for ductile creep rupture (i.e. strain induced softening leading to significant tertiary creep) an be dealt with simply by the same procedure existent in $\mathrm{NH}$ at present, i.e. it is assumed that an element of material at the most critical location in the structure is assumed to act like an independent test specimen, and its life predicted from data derived from creep rupture tests. This procedure is known to be conservative, and API 579 permits the use of considerably more detailed methods, including the effects of continuum damage, to take account of the phenomenon of damage distribution which is believed to occur when tertiary creep is a significant factor.

Brittle creep failure by local ductility exhaustion is also considered by API 579. All creep analyses include the effects of multiaxiality on both strain accumulation and fracture.

Creep/fatigue is dealt with by essentially the same procedure as is used in NH. No mention is made of "elastic follow-up". The guideline does not emphasize structural analysis methodology in its outline of assessment of creep/fatigue interaction but it provides monotonic, cyclic and isochronous stress/strain curves as a matter of course, and appears to assume $a$ priori that any assessment of creep/fatigue interaction will be based on a nonlinear, cyclic analysis of some form or other.

Creep crack growth from preexisting defects is considered, and can be adapted to include the history of creep damage evolution in local stress concentrations. An in-depth 
procedure is provided for combining the creep damage generation ahead of the crack (or defect, or SCF) leading to creep crack initiation and growth. This procedure borrows heavily from work done in relation to the development of R5 and reflects communications between members of the international FFS community. No design criteria are provided to assess radiation effects and associated failure mechanisms.

Thermal aging effects are limited to fatigue strength reduction factors. 
Table 9.2.6.1 API579: High Temperature

\begin{tabular}{|c|c|c|l|}
\hline Definition / Criterion & Covered? & Location & \multicolumn{1}{|c|}{ Comment } \\
\hline High temperature limit & Yes & Para 10.4.2 & $\begin{array}{l}\text { Significant creep defined by a Level 1 screening assesment } \\
\text { based on simplified creep damage accumulation curves } \\
\text { developed for specific materials }\end{array}$ \\
\hline $\begin{array}{c}\text { Criterion or definition of } \\
\text { temperature limit for } \\
\text { insignificant creep effects }\end{array}$ & No & & $\begin{array}{l}\text { Negligible creep is defined on a case-by-case basis using a } \\
\text { simplifie damage criterion outlined above }\end{array}$ \\
\hline
\end{tabular}


Table 9.2.6.2-1 API579: Design Loads

\begin{tabular}{|c|c|c|c|c|}
\hline ID\# & Definition / Criterion & Covered? & Location & Comment \\
\hline a & $\begin{array}{l}\text { Guidelines for load historgram \& } \\
\text { cycle definition }\end{array}$ & Yes & $\begin{array}{l}\text { Level } 1 \text { in 10.4.2.2 ; Levels 2, } 3 \text { in } \\
\text { 10.5.2.3 }\end{array}$ & $\begin{array}{l}\text { Extensive guidance given on the definition and implemation of hload histories for } \\
\text { assessment purposes. }\end{array}$ \\
\hline $\mathrm{b}$ & Glossary of terms & Yes & Para 10.9 and Annex I & Comprehensive listing of all terms and definitions \\
\hline c & Definition of stress intensities & Yes? & $\begin{array}{l}\text { Annex B1.2.2 and Annex B2 for } \\
\text { linearization procedures }\end{array}$ & $\begin{array}{l}\text { Comprehensive of stress clasification, including current ASME Code nethodolgy } \\
\text { as well as alternative procedures specifically designed for FE of complex } \\
\text { component geometries }\end{array}$ \\
\hline d & Definition of strain intensities & Yes & & Equivalent strain (Mises) is used rather than 'strain intensity'. \\
\hline $\mathrm{g}$ & Procedure for stress linearization & No & see c) above & see c) above \\
\hline
\end{tabular}


Table 9.2.6.2-2 API579: Design Loads

\begin{tabular}{|c|c|c|c|c|}
\hline ID\# & Definition / Criterion & Covered? & Location & Comment \\
\hline $\mathrm{h}$ & Differentiated load categories: & Yes & $\begin{array}{l}\text { Table B1.1; Tables B1.2 thru } \\
4\end{array}$ & $\begin{array}{l}\text { Different load categories recognized but the categories are more } \\
\text { specific to a known in-service loading history, as suited to an FFS } \\
\text { guideline }\end{array}$ \\
\hline & design loads & yes & see above & see above \\
\hline & normal service loads & yes & see above & see above \\
\hline & frequent abnormal loads & see above & see above & see above \\
\hline & infrequent abnormal loads & see above & see above & see above \\
\hline & limiting fault loads & see above & see above & see above \\
\hline & test loads & yes & see above & Test allowables \\
\hline $\mathrm{i}$ & $\begin{array}{r}\text { Flow chart for design by analysis } \\
\text { or rule/procedure }\end{array}$ & No & & Implicit acceptance of ASME procedure without citation \\
\hline j & $\begin{array}{r}\text { Yield function requirements (e.g. } \\
\text { Tresca vs Mises) }\end{array}$ & Yes & B1.2.2 & $\begin{array}{l}\text { Mises for plasticity and creep deformation; other rules for creep } \\
\text { damage }\end{array}$ \\
\hline
\end{tabular}


Table 9.2.6.3-1 API579: Failure Mechanisms

\begin{tabular}{|c|c|c|c|c|}
\hline ID\# & Definition / Criterion & Covered? & Location & Comment \\
\hline $\mathrm{a}$ & $\begin{array}{l}\text { Limit load collapse, single load } \\
\text { application }\end{array}$ & Yes & B1.2 & $\begin{array}{l}\text { Method follows ASME VIII/2 but application spelt out in detail in } \\
\text { fomr of algorithm }\end{array}$ \\
\hline $\mathrm{b}$ & $\begin{array}{l}\text { Excessive deformation limiting } \\
\text { functionality, under a single load } \\
\text { application }\end{array}$ & ? & & $\begin{array}{l}\text { Covered implicitly by permitted detailed FE procedures including } \\
\text { both plasticity and creep simultaneously }\end{array}$ \\
\hline c & $\begin{array}{l}\text { Structural instability or buckling, } \\
\text { under a single load application }\end{array}$ & Yes & B1.4;para 10.5.5 & $\begin{array}{l}\text { Short term buckling based on full inelastic analysis; special creep } \\
\text { procedure limited to tubes under external pressure }\end{array}$ \\
\hline d & $\begin{array}{l}\text { Progressive collapse by } \\
\text { ratcheting under cyclic load }\end{array}$ & Yes & B1.5.6 & $\begin{array}{l}\text { Two methods offered for short term (no creep); a simplified elastic } \\
\text { based method using cyclic FEA and detailed full inelastic cyclic } \\
\text { analysis which could, in principle be used also for creep analysis }\end{array}$ \\
\hline e & Nonductile fracture & Yes & B1.3.3 & $\begin{array}{l}\text { Creep ductility, including multiaxial effects considered explicitly as } \\
\text { part of Elastic/plastic analysis }\end{array}$ \\
\hline $\mathrm{f}$ & $\begin{array}{l}\text { Fatigue failure (nucleation on } \\
\text { order of } 5 \mathrm{~mm} \text { ) }\end{array}$ & Yes & B1.5 & $\begin{array}{l}\text { Extensive in-depth treatment of both structural fatigue analysis } \\
\text { together with material data; includes bth initiation and crack } \\
\text { propagation with material data to support both }\end{array}$ \\
\hline g & $\begin{array}{l}\text { Collapse or breach of pressure } \\
\text { boundary due to corrosion, mass } \\
\text { transfer phenomenon, etc. }\end{array}$ & Yes & Parts 4,5,6,7 & $\begin{array}{l}\text { Extensive coverage of all aspects of corrosion including genral } \\
\text { wasting, local pitting and SCC; corroded sections iclued as routine } \\
\text { matter in stuctural analysis }\end{array}$ \\
\hline $\mathrm{h}$ & $\begin{array}{l}\text { Excessive deformation leading to } \\
\text { loss of functionality, due to creep } \\
\text { under steady load }\end{array}$ & Yes & B1.3.3 & $\begin{array}{l}\text { Not treated separately but considered a integral part of cyclic } \\
\text { inelastic analysis }\end{array}$ \\
\hline
\end{tabular}


Table 9.2.6.3-2 API579: Failure Mechanisms

\begin{tabular}{|c|l|c|c|l|}
\hline ID\# & Definition / Criterion & Covered? & Location & \multicolumn{1}{|c|}{ Comment } \\
\hline $\mathrm{i}$ & Creep rupture & Yes & para 10.5.3;Annex f7 & $\begin{array}{l}\text { Creep rupture dealt with in detail using MPC/Omega } \\
\text { model, tied to comprehensive material database for both } \\
\text { creep strain and damage computaion; includes LMP data } \\
\text { on most common materials from API 530 database in } \\
\text { parametric form }\end{array}$ \\
\hline $\mathrm{j}$ & Creep buckling & Yes & para 10.23 & $\begin{array}{l}\text { recommended method for creep buckling limited to } \\
\text { tubes; other methods permitted but not defined }\end{array}$ \\
\hline $\mathrm{k}$ & Enhanced creep & Yes & B1.3.3 & $\begin{array}{l}\text { Not treated a separate phenomenon, but considered part } \\
\text { of integrated cyclic inelastic analysis }\end{array}$ \\
\hline $\mathrm{l}$ & Creep-Fatigue & Yes & para 10.5.3; Annex F7 & Follows general NH procedure \\
\hline $\mathrm{m}$ & Accelerated creep rupture & No & para 10.5.3; Annex F7 & Follows general NH procedure \\
\hline $\mathrm{n}$ & $\begin{array}{l}\text { Softening enhanced cyclic creep } \\
\text { rupture }\end{array}$ & No & & \\
\hline $\mathrm{o}$ & $\begin{array}{l}\text { Softening enhanced cyclic creep } \\
\text { deformation }\end{array}$ & No & & \\
\hline $\mathrm{p}$ & Irradiation effects & No & & \\
\hline
\end{tabular}


Table 9.2.6.3-3 API579: Failure Mechanisms

\begin{tabular}{|c|l|c|l|l|}
\hline ID\# & \multicolumn{1}{|c|}{ Definition / Criterion } & Covered? & Location & Comment \\
\hline q & weldments (strength, strain) & Yes & See comment next column & $\begin{array}{l}\text { Too many references to list; extensive treatement of all aspects of } \\
\text { welds and weldments; weldment properties listed in Annex F }\end{array}$ \\
\hline r & $\begin{array}{l}\text { Multiaxial effects on creep, fatigue, } \\
\text { C-F }\end{array}$ & Yes & $\begin{array}{l}\text { para 10.5.2 (rupture);para 10.5.3. } \\
\text { creep/fatigue interaction }\end{array}$ & $\begin{array}{l}\text { Detailed methods for creep rupture assessment include multiaxial } \\
\text { stress state; creep/fatigue interaction procedure assumed to be } \\
\text { based on FEA and automatically introduces general stress state }\end{array}$ \\
\hline s & Local vs. distributed damage & Yes & See comment next column & $\begin{array}{l}\text { API 579 is drafted with the understanding that a Level 3 } \\
\text { assessment, which includes damage, will include creep rupture, } \\
\text { local creep damage leading to cracking, and creep crack } \\
\text { propagation; detailed procedures exist to deal with all these } \\
\text { mechanisms with the associated material data provided in Annex F }\end{array}$ \\
\hline $\mathrm{t}$ & Thermal aging & Yes & Para 10.4.2; Annex F, & $\begin{array}{l}\text { Level 1 screening assessment uses BHN testing as first level to } \\
\text { determine creep damage; creep database is derived from service } \\
\text { exposed material }\end{array}$ \\
\hline $\mathrm{u}$ & Elastic follow-up & No & $\begin{array}{l}\text { This simplifying concept is supplanted in API 579 by analyses of } \\
\text { sufficient complexity to make it redundant }\end{array}$ \\
\hline
\end{tabular}


Table 9.2.6.4-1 API579: Design Criteria / Procedures

\begin{tabular}{|c|c|c|c|c|c|c|c|c|c|}
\hline 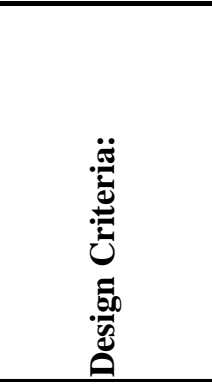 & 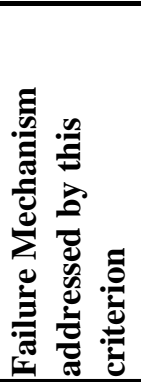 & 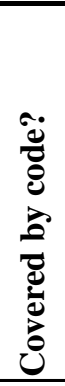 & 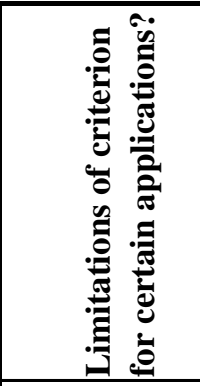 & 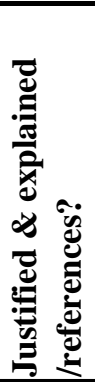 & 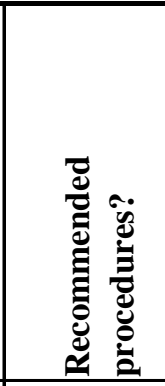 & 晜 & 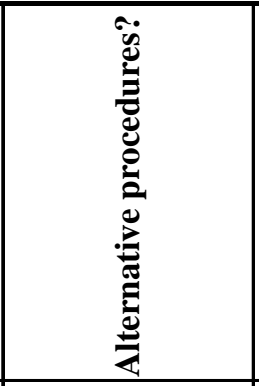 & 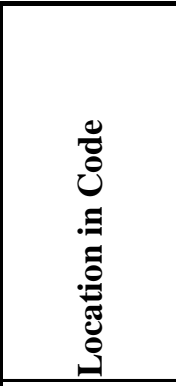 & 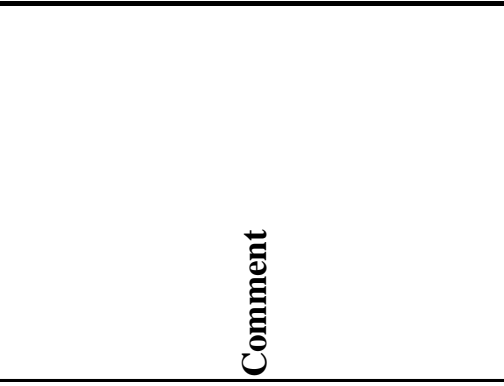 \\
\hline i. Limit Load & a & Yes & No. & Yes & Yes & No & $\begin{array}{l}\text { Reference stress } \\
\text { solutions for simple } \\
\text { geometries are listed } \\
\text { in Part } 8 \text {. More } \\
\text { complex solutions } \\
\text { are assumed to be } \\
\text { found by detailed } \\
\text { FEA. }\end{array}$ & $\begin{array}{l}\text { Part 8, Annex } \\
\text { B-1 }\end{array}$ & $\begin{array}{l}\text { Criteria similar to Section III NB. Not } \\
\text { employed for ETD }\end{array}$ \\
\hline $\begin{array}{l}\text { ii. Excessive } \\
\text { deflection }\end{array}$ & $\mathrm{b}$ & No. & See comments & & & & & & $\begin{array}{l}\text { API } 579 \text { does not address excessive } \\
\text { deflection explicitly. Since it is a FFS } \\
\text { guideline, the need to consider } \\
\text { deformation as a degradation of function } \\
\text { appears to be left to the analyst }\end{array}$ \\
\hline iii. Buckling & c & Yes & $\begin{array}{l}\text { Simple rules for } \\
\text { tubes }\end{array}$ & Yes & No & No & Yes & Part 10 & $\begin{array}{l}\text { Simle tubes use Chern solution. Otherwise } \\
\text { nonlinear FE avocated }\end{array}$ \\
\hline iv. Ratcheting & $3 d$ & Yes. & No & Yes. & $\begin{array}{l}\text { Direct FEA, } \\
\text { either } \\
\text { simplified } \\
\text { elastic or full } \\
\text { inelastic } \\
\text { analysis }\end{array}$ & No & None specified. & Annex B1 & $\begin{array}{l}\text { Ratcheting, with and without creep is } \\
\text { assumed to be best evaluated by direct } \\
\text { cyclic analysis. Procedures available in } \\
\text { other accepted international codes can be } \\
\text { used }\end{array}$ \\
\hline
\end{tabular}


Table 9.2.6.4-2 API579: Design Criteria / Procedures

\begin{tabular}{|c|c|c|c|c|c|c|c|c|c|}
\hline 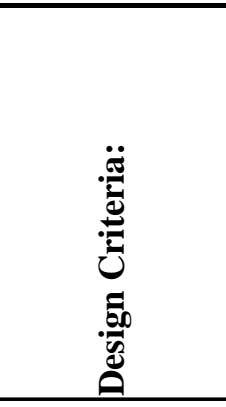 & 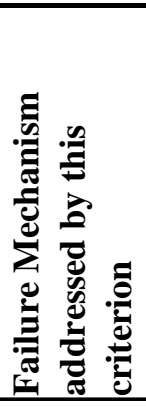 & 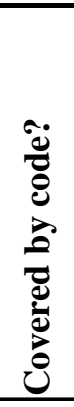 & 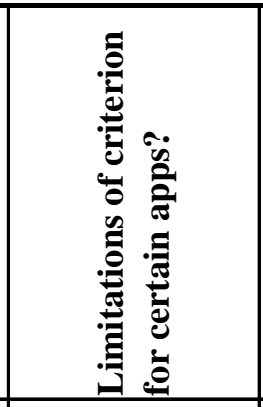 & 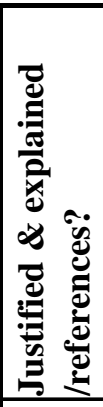 & 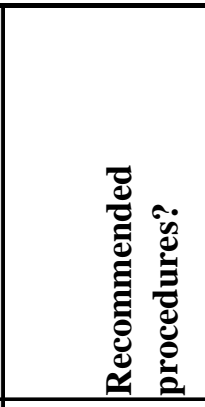 & 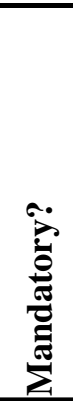 & 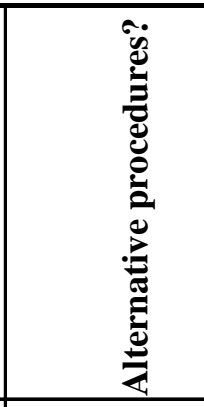 & 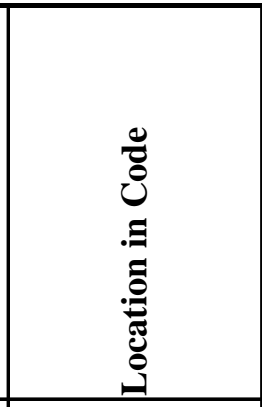 & 苞 \\
\hline iv. Ratcheting & d & Yes & No limitation & Yes & Yes & no & Yes & B1.3.3 & $\begin{array}{l}\text { No simplified method like Bree } \\
\text { diagram; two methods for direct } \\
\text { cyclic analysis, one simplified } \\
\text { and one full detail }\end{array}$ \\
\hline v. Fracture & e & Yes & No limitation & Yes & Yes & No & Yes & $\begin{array}{l}\text { Part } 3 \text { Brittle } \\
\text { fracture; part } 9 \\
\text { assessment of } \\
\text { cracks; Annex C } \\
\text { compendium of SI } \\
\text { solutions; Annex D: } \\
\text { Annex F materials }\end{array}$ & $\begin{array}{l}\text { As a FFS guideline API } 579 \\
\text { contains comprehensive } \\
\text { treatment of all aspects of } \\
\text { fracture }\end{array}$ \\
\hline vi. Fatigue & $\mathrm{f}$ & Yes. & No limitation & Yes & Miner's rule. & Yes. & None apparent & see "e" above & see "e" above \\
\hline vii. Corrosion & g & Yes & \begin{tabular}{|l|} 
primary concern \\
with geometric \\
results of corrosion
\end{tabular} & Yes & Yes & Yes & Yes & Parts 4,5,6,7 & $\begin{array}{l}\text { As a FFS guideline API } 579 \\
\text { contains comprehensive } \\
\text { treatment of all aspects of } \\
\text { corrosion }\end{array}$ \\
\hline
\end{tabular}


Table 9.2.6.4-3 API579: Design Criteria / Procedures

\begin{tabular}{|c|c|c|c|c|c|c|c|c|c|}
\hline 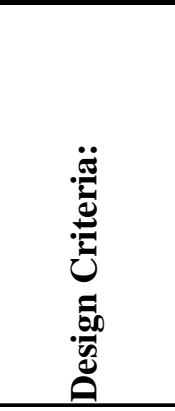 & 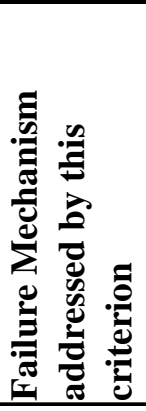 & 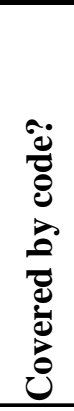 & 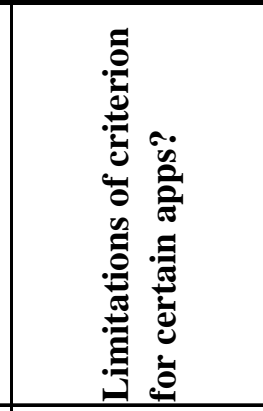 & 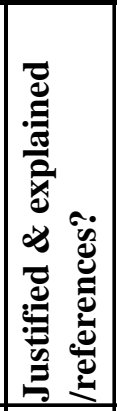 & 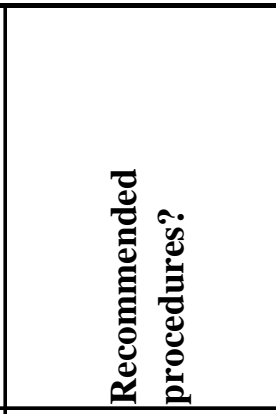 & 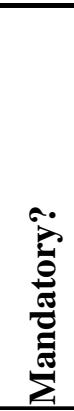 & 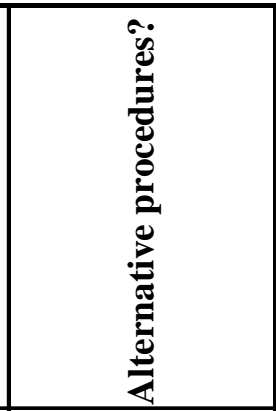 & 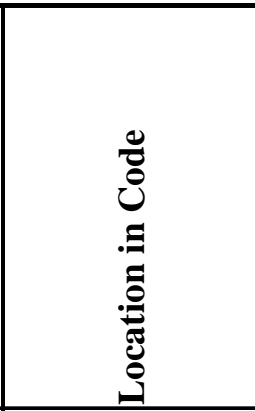 & 苞 \\
\hline $\begin{array}{c}\text { viii. } \\
\text { Excessive } \\
\text { creep } \\
\text { deformation } \\
\end{array}$ & $\mathrm{h}$ & Yes. & $\begin{array}{l}\text { Strain limitations, } \\
\text { not structural } \\
\text { deformation limits }\end{array}$ & Yes. & $\begin{array}{l}\text { Essentially the same } \\
\text { procdure as NH }\end{array}$ & Yes. & no & & $\begin{array}{l}\text { Strain is limited to } 1 \%, 2 \% \text { \& } \\
5 \% \text {, AND stress intensity is } \\
\text { dictated by limiting strain to } 1 \%\end{array}$ \\
\hline $\begin{array}{l}\text { ix. Creep } \\
\text { buckling }\end{array}$ & h,b & Yes & $\begin{array}{l}\text { Standard methods } \\
\text { for tubes under } \\
\text { external pressure; } \\
\text { detailed analysis } \\
\text { for other } \\
\text { geometries }\end{array}$ & Yes & Yes & No & Yes & Para 10.5 .5 & $\begin{array}{l}\text { Chern method provided for } \\
\text { externally pressurized tubes; } \\
\text { detailed inelastic analysis for all } \\
\text { else }\end{array}$ \\
\hline $\begin{array}{l}\text { x. Creep } \\
\text { rupture }\end{array}$ & 3b,h,i & Yes. & $\begin{array}{l}\text { Omega method } \\
\text { with associated } \\
\text { material properties } \\
\text { offered; other } \\
\text { methods optional }\end{array}$ & Yes. & Omega method & Yes. & $\begin{array}{l}\text { Any internationally } \\
\text { recognized HT code } \\
\text { procedure is } \\
\text { acceptable as } \\
\text { alternaive to Omega } \\
\text { method }\end{array}$ & para 10.5.2;B1.3.3 & $\begin{array}{l}\text { Major feature of API } 579 \text { and } \\
\text { dealt with extensively }\end{array}$ \\
\hline
\end{tabular}


Table 9.2.6.4-4 API579: Design Criteria / Procedures

\begin{tabular}{|c|c|c|c|c|c|c|c|c|c|}
\hline 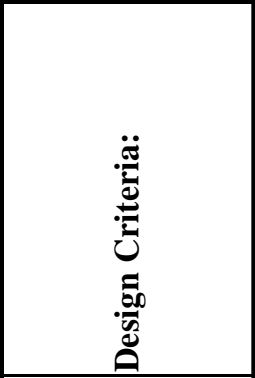 & 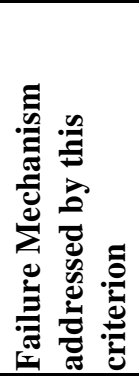 & 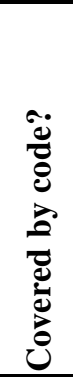 & 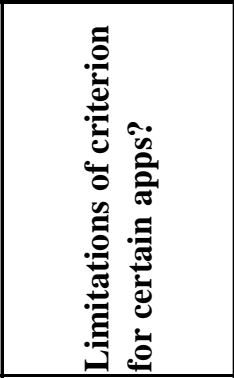 & 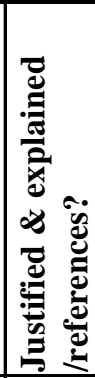 & 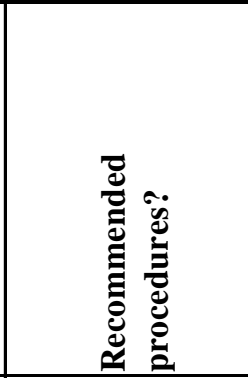 & 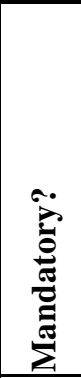 & 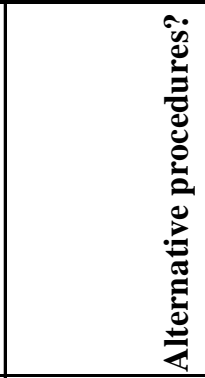 & 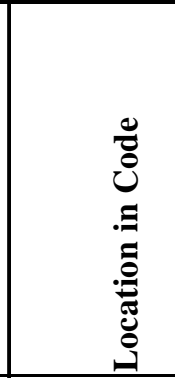 & 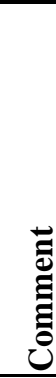 \\
\hline 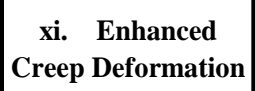 & $\mathrm{j}$ & No & & & & & & & \\
\hline $\begin{array}{c}\text { xii. C-F } \\
\text { Interaction }\end{array}$ & $3 \mathrm{k}$ & Yes. & $\begin{array}{l}\text { Linear damage rule } \\
\text { limitations. }\end{array}$ & Yes. & Linear damage rule. & Yes. & no & Section 3.4.2 & \\
\hline
\end{tabular}


Table 9.2.6.4-5 API579: Design Criteria / Procedures

\begin{tabular}{|c|c|c|c|c|c|c|c|c|c|}
\hline 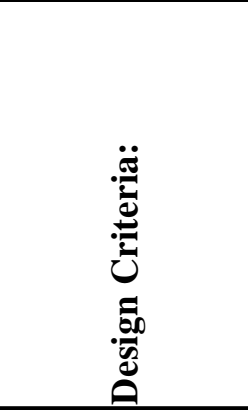 & 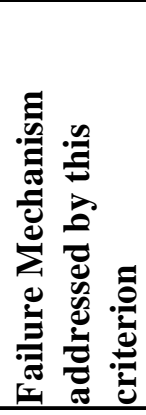 & 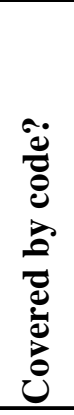 & 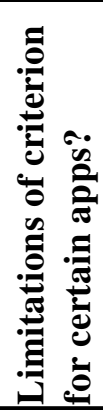 & 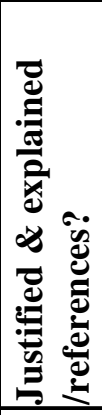 & 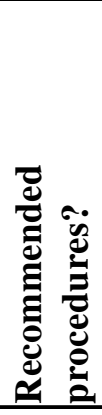 & 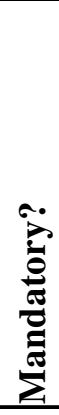 & 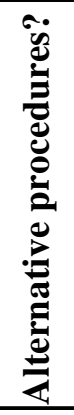 & 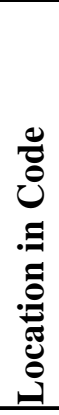 & 晜 \\
\hline $\begin{array}{l}\text { xiii. Accelerated } \\
\text { Cyclic Creep } \\
\text { Rupture }\end{array}$ & l & No & & & & & & & \\
\hline $\begin{array}{c}\text { xiv. Softening } \\
\text { Enhanced Cyc. } \\
\text { Creep } \\
\text { Deformation }\end{array}$ & $\mathrm{m}$ & No. & & & & & & & \\
\hline $\begin{array}{l}\text { xv. Softening } \\
\text { Enhanced Cyc. } \\
\text { Creep Rupture }\end{array}$ & $\mathrm{n}$ & No. & & & & & & & \\
\hline
\end{tabular}


Table 9.2.6.4-6 API579: Design Criteria / Procedures

\begin{tabular}{|c|c|c|c|c|c|c|c|c|c|}
\hline 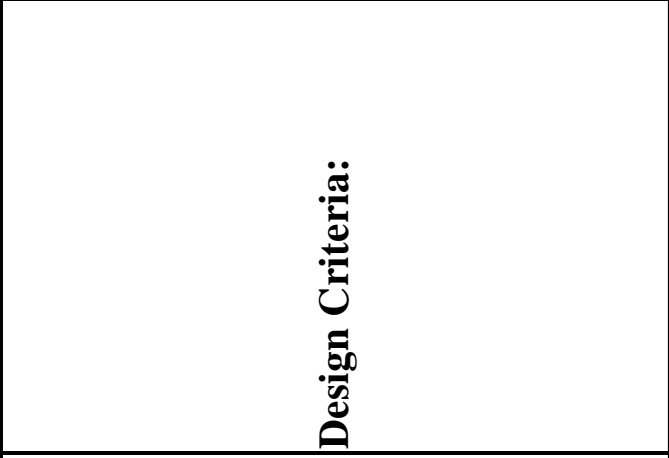 & 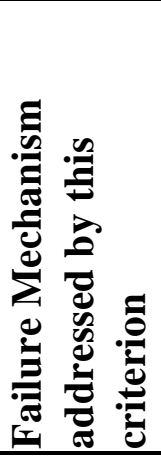 & 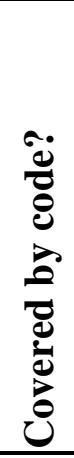 & 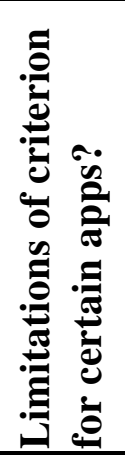 & 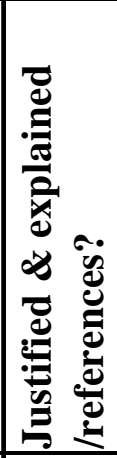 & 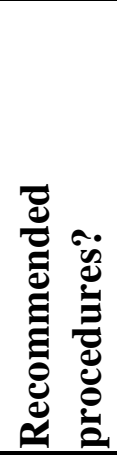 & 苛 & 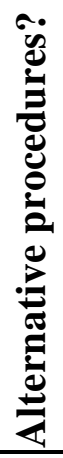 & 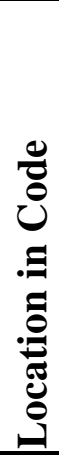 & $\begin{array}{l}\ddot{\Xi} \\
\dot{\Xi} \\
0\end{array}$ \\
\hline xvi. Irradiation induced swelling & o & No. & & & & & & & \\
\hline xvii. Irradiation induced creep & 0 & No. & & & & & & & \\
\hline $\begin{array}{l}\text { xviii. Plastic Flow localization due to } \\
\text { irradiation (dislocation tunneling) }\end{array}$ & 0 & No. & & & & & & & \\
\hline $\begin{array}{c}\text { xix. Ductility exhaustion due to irradiation } \\
\text { exposure }\end{array}$ & 0 & No. & & & & & & & \\
\hline $\begin{array}{c}x x . \quad \text { Irradiation induced effects on fatigue, } \\
\text { and on C-F }\end{array}$ & 0 & No. & & & & & & & \\
\hline
\end{tabular}


Table 9.2.6.4-7 API579: Design Criteria / Procedures

\begin{tabular}{|c|c|c|c|c|c|c|c|c|c|}
\hline 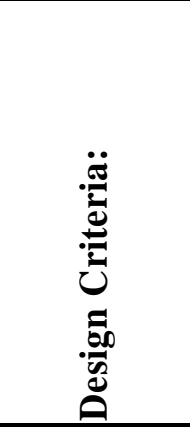 & 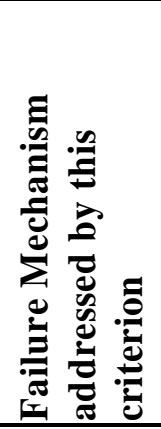 & 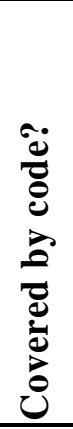 & 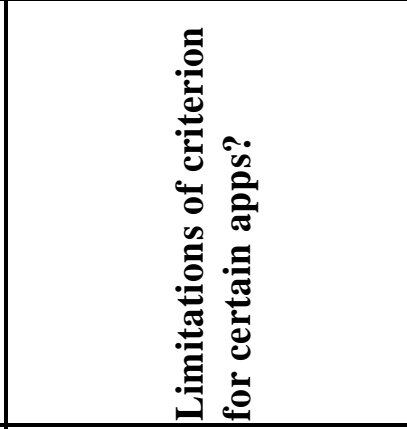 & 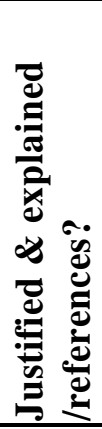 & 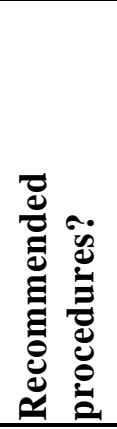 & 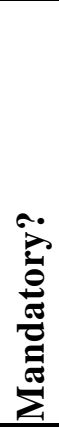 & 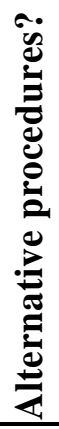 & 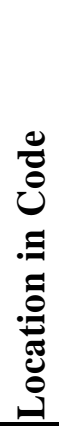 & 葛 \\
\hline $\begin{array}{l}\text { xxi. Local } \\
\text { vs. non-local } \\
\text { damage }\end{array}$ & $\mathrm{p}$ & No & & & & & & & \\
\hline $\begin{array}{c}\text { xxii. } \\
\text { Thermal } \\
\text { aging effects } \\
\text { on creep, } \\
\text { fatigue, limit } \\
\text { load }\end{array}$ & $\mathrm{q}$ & No & & & & & & & \\
\hline $\begin{array}{c}\text { xxiii. } \\
\text { Elastic } \\
\text { Follow-up }\end{array}$ & $\mathrm{r}$ & No & $\begin{array}{l}\text { Redundant concept in API } 579 \\
\text { since this analysis assumed to } \\
\text { be done by direct analysis }\end{array}$ & & & & & & \\
\hline
\end{tabular}




\subsubsection{Summary}

Several International ETD Codes were reviewed and compared relative to the Ideal ETD Code described in Subtask 9.1. The review followed the format of the Ideal ETD Code, namely High Temperature, Design Loads, Failure Mechanisms, and Design

Criteria/Procedures. Tables were utilized to summarize the extent by which the various Codes addressed elements of the Ideal ETD Code; accompanying details are provided to highlight the various Code approaches, limitations, and relevant locations in the Codes. The intent being to provide future designers, Code developers, and regulators such as the NRC with a roadmap to each Code to more readily understand the breadth of coverage in each. No attempt was made to compare the Codes with one another, since Subtask 9.3 will specifically address the evaluation of each International Code relative to the ASME NH Code. 


\title{
Task 9: Update and Improve Subsection NH - Simplified Elastic and Inelastic Design Analysis Methods
}

\section{Subtask 9.3 - Comparison of Current International ETD Codes}

\author{
Gen IV / NGNP Materials Project \\ Submitted to \\ Mr. Jim Ramirez \\ Vice President, Business Development \\ ASME Standards Technology, LLC \\ Three Park Avenue \\ New York, NY 10016 USA \\ By \\ Advanced Consulting Engineering Services, Inc. \\ Peoria, IL \\ On behalf of \\ Jeries Abou-Hanna \\ Douglas L. Marriott \\ Timothy E. McGreevy
}

December 31, 2008 


\section{TABLE OF CONTENTS}

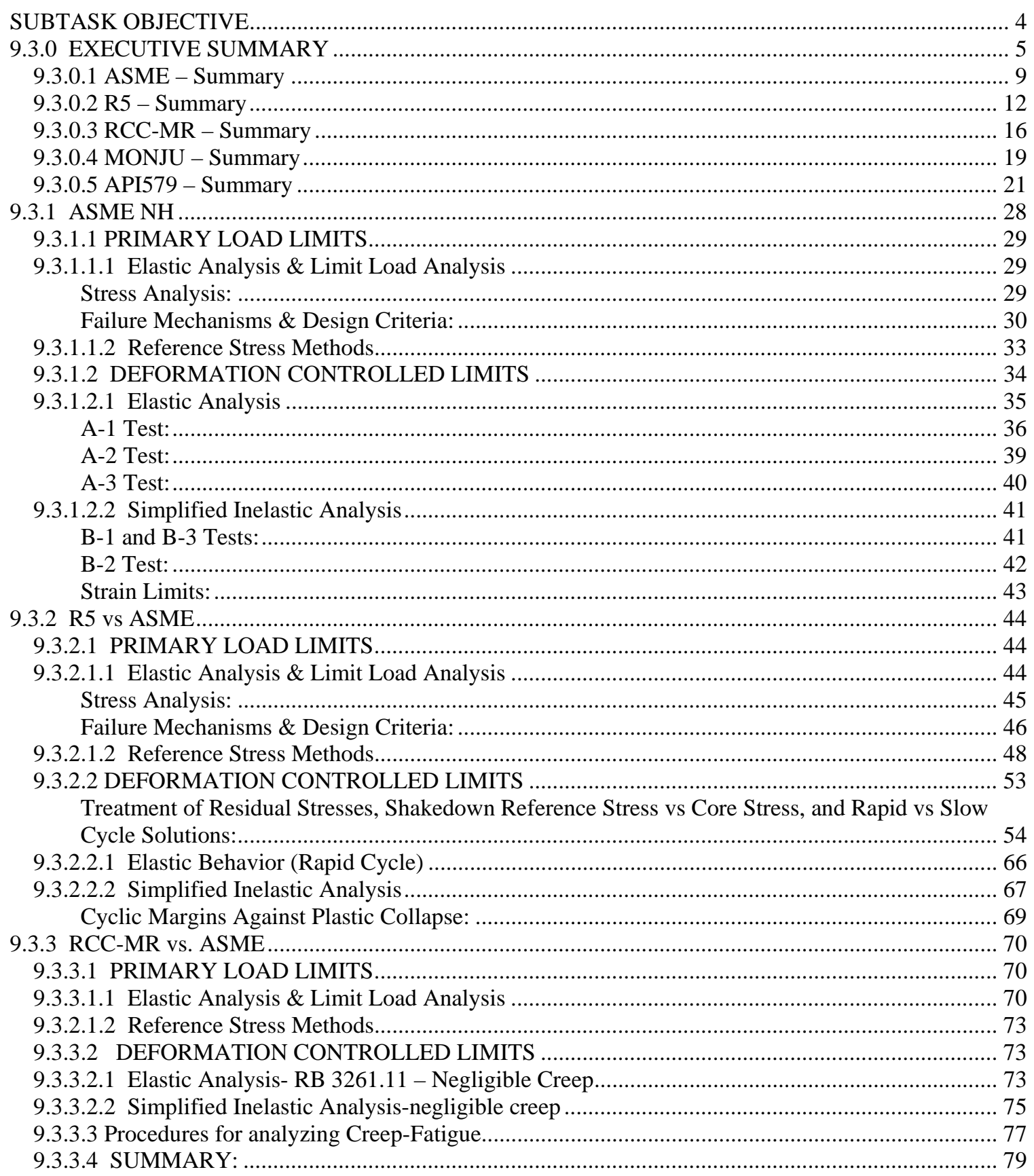




\section{TABLE OF CONTENTS (continued)}

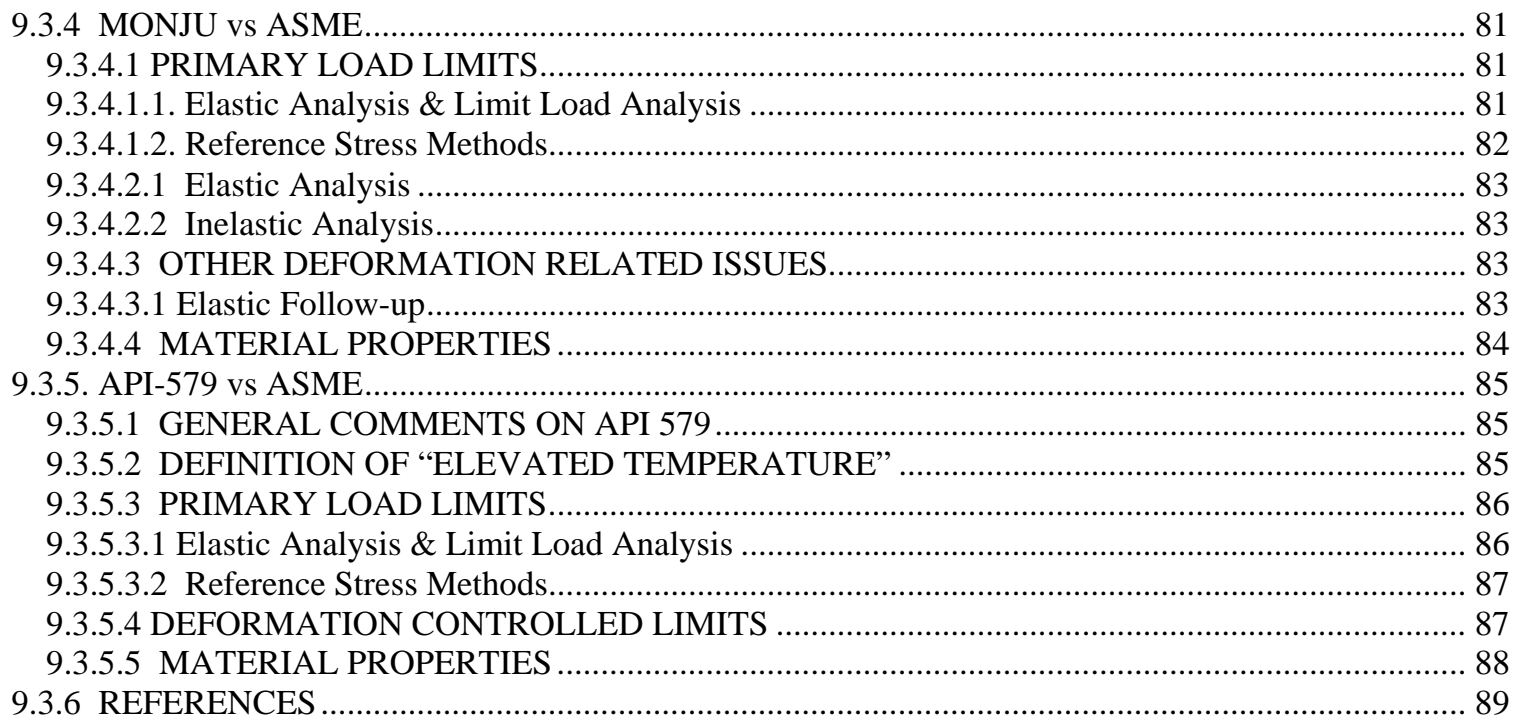




\section{SUBTASK OBJECTIVE}

The objective of this subtask is to provide a comparison of ASME, RCC-MR, R5, Monju, and API-579 Codes. This review will take into account any experience and perspective of the TIs, discussion/interviews with other International ETD experts, a review of published literature regarding existing Code rules, and a review of International ETD Codes provided in an earlier report for Subtask 9.2. Specifically, the TIs will identify and summarize both strengths and weaknesses in current methods and assessment procedures in the existing International ETD Code outlined above. This report differs from Subtask 9.2 in that 9.2 compared the International ETD Codes to the Ideal ETD Code specified in Subtask 9.1.

Where possible, the strengths and weaknesses will be illustrated with comparative examples obtained from the literature; the scope of these comparisons will be limited to

- Elastic analysis,

- Reference stress methods,

- Limit load, shakedown, and ratcheting analysis.

Note, these three areas are a subset of the Ideal ETD Code and all International ETD Codes discussed and reported in Subtasks 9.1 and 9.2. These topics are addressed in terms of Primary Load Limits (elastic analysis, reference stress methods, and limit load analysis) and Deformation Controlled Limits (elastic analysis, shakedown and ratcheting analysis, and the use of limit load and/or reference stress methods to address elastic/shakedown/plasticity/ratcheting analysis.

The ASME NH Code is summarized first. Subsequent ETD Codes are compared with the ASME NH Code. No direct comparison between non ASME ETD Codes is made; for instance, a comparison between RCC-MR and R5 would provide limited to no value as many of the foundations from the ETD Codes are based upon similar if not identical 
fundamental principals. Rather, any relevant differences between International Codes other than ASME will be readily apparent.

\subsubsection{EXECUTIVE SUMMARY}

While the review of the International ETD Codes is restricted to elastic analysis, reference stress, and shakedown/ratcheting/limit analysis, the details of such topics can easily overwhelm the reader, especially when one considers a review of ASME, RCCMR, R5, Monju, and API 579. Hence, the authors present this summary in order to provide the reader with a concise picture of the comparison of International ETD Codes. The format of the report provides a means for quickly locating topics in each International Code, and easy reference to the executive summary. The intent is to aid the reader in maintaining the broader view, while permitting examination of details as desired.

Table 9.3.0.1 provides a summary of ASME ETD Code assessment from this review. Details of the review are provided in section 9.3.1. The table is organized according to primary load limits, including both time independent and time dependent, and deformation controlled limits. Appropriate 'categories' are found in the first column, e.g. elastic or inelastic. Categories are common between time dependent and independent primary load limits, while additional categories are specified for deformation controlled limits. A 'rank' is provided for each category, ranging from the lowest to best score of: 'not allowed', 'not addressed', 'inadequate', 'adequate', 'effective', and 'excellent'. 'Not allowed' and 'not addressed' are self-explanatory. 'Inadequate' reflects the authors' opinions that the Code attempts to address the topic but does not provide sufficient or technically appropriate guidance. 'Adequate' indicates that in the authors' opinions the Code provides sufficient and appropriate guidance. 'Effective' indicates that the guidance is more comprehensive, e.g. applicable to general structures and loading and not simply ideal/simple geometries and loading conditions, and may also provide various options that result in less conservative designs but maintain an appropriate level of conservatism 
than 'adequate'. 'Excellent' indicates that the Code is very comprehensive, and as such may also provide options for varying levels of conservatism.

Table 9.3.0.2 provides a summary of International ETD Codes relative to ASME ETD Code (ASME NH). The same categories are used as in Table 9.3.0.1. ASME's rank is provided, and a relative rank is provided for R5, RCC-MR, Monju, and API 579. The 'key' to the relative rankings is provided and interpreted as follows. If ASME addresses the category, i.e. if ASME is anything other than 'NOT ALLOWED' or 'NOT ADDRESSED', then three pluses indicates 'superior' relative to ASME, and so on according to the key. If ASME's rank is 'NOT ALLOWED' or 'NOT ADDRESSED', then the ranking provided is not a relative ranking; as such, three pluses indicates 'excellent', two pluses 'effective', and so on. A broad summary of each ETD Code is provided below, with the intent to accompany the use and interpretation of Tables 9.3.0.1 and 9.3.0.2. 
Table 9.3.0.1 Summary of ASME NH Code Assessment

\begin{tabular}{|c|c|c|}
\hline \multicolumn{3}{|c|}{ SUMMARY TABLE: ASME NH CODE ASSESSMENT } \\
\hline CATEGORY & RANK & COMMENT \\
\hline \multicolumn{3}{|c|}{ PRIMARY LOAD LIMITS -TIME INDEPENDENT } \\
\hline ELASTIC & EFFECTIVE & STRESS CLASSIFICATION DIFFICULT \\
\hline INELASTIC & NOT ALLOWED & \\
\hline LIMIT LOAD METHOD & NOT ALLOWED & \\
\hline $\begin{array}{l}\text { JUSTIFICATION \& } \\
\text { DOCUMENTATION }\end{array}$ & NO JUSTIFICATION & \\
\hline \multicolumn{3}{|c|}{ PRIMARY LOAD LIMITS -TIME DEPENDENT } \\
\hline ELASTIC & EFFECTIVE & STRESS CLASSIFICATION DIFFICULT \\
\hline INELASTIC & NOT ALLOWED & \\
\hline LIMIT LOAD METHOD & NOT ALLOWED & \\
\hline $\begin{array}{l}\text { JUSTIFICATION \& } \\
\text { DOCUMENTATION }\end{array}$ & NO JUSTIFICATION & \\
\hline \multicolumn{3}{|c|}{ DEFORMATION CONTROLLED LIMITS } \\
\hline ELASTIC FOLLOW-UP & ADEQUATE & \\
\hline ELASTIC ANALYSIS & ADEQUATE & $\begin{array}{l}\text { STRESS CLASSIFICATION DIFFICULT I } \\
\text { BREE LIMITATIONS }\end{array}$ \\
\hline INELASTIC ANALYSIS & INADEQUATE & NO GUIDANCE \\
\hline LIMIT LOAD METHOD & NOT ADRESSED & \\
\hline $\begin{array}{l}\text { JUSTIFICATION \& } \\
\text { DOCUMENTATION } \\
\end{array}$ & NO JUSTIFICATION & \\
\hline CYCLE DEFINITION & INADEQUATE & UNCLEAR; LIMITED DESCRIPTION \\
\hline RAPID VS. SLOW CYCLES & EFFECTIVE & NOT WELL DEFINED \\
\hline
\end{tabular}


Table 9.3.0.2 Summary of International ETD Codes vs ASME ETD Code

\begin{tabular}{|c|c|c|c|c|c|c|c|c|}
\hline \multicolumn{2}{|c|}{ SUMMARY TABLE: COMPARISON TO ASME } & \multicolumn{4}{|c|}{ OTHER CODES } & & & \\
\hline \multicolumn{6}{|c|}{ PRIMARY LOAD LIMITS -TIME INDEPENDENT } & & & \\
\hline ELASTIC & EFFECTIVE & 0 & 0 & 0 & + & & & \\
\hline $\begin{array}{ll}\text { Ling } \\
\end{array}$ & NOT ALLOWED & +++ & ++ & $0 /++$ & + & & & \\
\hline $\begin{array}{l}\text { JUSTIFICATION \& } \\
\text { DOCUMENTATION }\end{array}$ & NO JUSTIFICATION & +++ & + & 0 & +++ & & & \\
\hline INELASTIC & NOT ALLOWED & ++ & ++ & $0 /++$ & +++ & ++ & SIGNIFICANTLYBETTER & EFFECTIVE \\
\hline 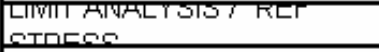 & NOT ALLOWED & +++ & ++ & $0 /++$ & + & + & BETTER & ADEQUATE \\
\hline $\begin{array}{l}\text { JUSTIFICATION \& } \\
\text { DOCUMENTATION } \\
\end{array}$ & NO JUSTIFICATION & +++ & 0 & 0 & +++ & 0 & SAME & \\
\hline \multicolumn{6}{|c|}{ DEFORMATION CONTROLLED LIMITS } & - & WORSE & INADEQUATE \\
\hline ELASTIC FOLLOW-UP & ADEQUATE & ++ & 0 & $+/ ?$ & Not Used & -- & SIGNIFICANTLYWORSE & \\
\hline CYCLE DEFINITION & INADEQUATE & + & + & 0 & +++ & & & \\
\hline RAPID VS. SLOW CYCLES & EFFECTVVE & $\ldots$ & 0 & 0 & Not Used & & & \\
\hline
\end{tabular}




\subsubsection{ASME - Summary}

\section{Primary Load Limits - Time Independent:}

Elastic: ASME's definition of primary and secondary stresses as well as peak stresses was revolutionary for structural design. The subsequent development and requirement of different design criteria were as well. Two main points limited the rank to 'EFFECTIVE' rather than 'EXCELLENT' and they are: issues with stress classifications, and modern engineering tools such as nonlinear finite element analysis (FEA).

Inelastic: ASME does not permit inelastic analysis when satisfying primary load limits for structures which operate at elevated temperature.

Limit Load Method: Similarly, ASME does not permit limit load analysis when satisfying primary load limits for structures which operate at elevated temperature.

Justification \& Documentation: The context of the use of the term 'Justification\& Documentation' must be clarified; the use of the term is with regards to whether or not the ETD Code provides references to work in the literature, National Laboratory reports, or other sources that provide detailed technical background to justify analysis procedures, material allowables, and/or design criteria. A 'NO JUSTIFICATION' does not indicate that no justification exists, rather only that such justification is not provided by the Code, and likely not conveniently within relevant sections of the Code where a user may find such relevant information useful. ASME does not provide such justification and documentation in this context.

\section{Primary Load Limits - Time Dependent:}

Elastic: ASME recognizes that stress redistribution will take place with creep deformation. The use of stress factors to reduce 'outer fiber' stresses is provided as the only option to address such redistribution of stresses. Similar to the time independent rank, two main points limited the rank to 'EFFECTIVE' rather than 'EXCELLENT'; 
these two points are the same as in the time independent case: issues with stress classifications, and modern engineering tools such as nonlinear finite element analysis (FEA).

Inelastic: ASME does not permit inelastic analysis when satisfying primary load limits for structures which operate at elevated temperature.

Limit Load Method: Similarly, ASME does not permit limit load analysis when satisfying primary load limits for structures which operate at elevated temperature.

Justification \& Documentation: ASME does not provide 'justification and documentation'.

\section{Deformation Controlled Limits:}

Elastic Follow-Up: ASME recognizes elastic follow-up. The effect of elastic follow-up is treated differently depending upon what type of design criteria and failure mechanism is being addressed. Experience with typical pressure vessels has led to the use of Tables to assist in classification of stresses in Primary Load Limits, while thermal membrane stresses are treated as primary stresses for ratcheting analysis. Elastic follow-up is considered infinite in determination of the creep strain increment when assessing strain range in fatigue analysis; however, stress is permitted to relax (elastic follow-up governed by deformation control, not load control) when assessing creep damage during cyclic loading. While the elastic follow-up factor may need to be treated differently depending upon the design criterion and failure mechanism of interest, the authors find the treatment to be 'adequate'. Stress classification issues contribute to this finding, as well as the extensive use of the ideal 'Bree tube' problem. While modern FEA permit more advanced assessment of elastic follow-up, ASME provides no guidance for such.

Elastic Analysis: The comments made with regards to elastic follow-up apply to the use of elastic analysis. Elastic analysis in this context refer to the use of elastic stress 
analysis and stress classification with results applied to Bree interaction diagrams for elastic, shakedown and ratcheting analysis, i.e. the A \& B Tests in Appendix T. Limitations for applicability to general structures and secondary loads other than linear thermal bending stresses exist as well.

Inelastic Analysis: Appendix T of ASME NH does permit inelastic analysis. In fact, it requires it for buckling analysis of structures made of Mod9Cr1Mo; that is, at higher temperatures, unified constitutive equations, which do not distinguish between ratedependent plasticity and time-dependent creep, should be used for satisfying the buckling limits of T-1520. No guidance is provided for conducting inelastic analysis. It is noted, that this is understood to be intentional, so as to not limit the user since the state of such analysis was limited at the time this portion of the Code was updated. Considerable advancement has taken place in conducting inelastic analysis; a summary of a variety of such methods is lacking. Implications of the use of various methods and application to design criteria and failure mechanisms are limited. Hence, a rank of 'inadequate’ was given.

Limit Load Method: Limit load analysis and limit analysis is an application of inelastic analysis, which can be applied to both monotonic loading of structures, e.g. primary load limits, as well as to cyclic loading of structures, e.g. shakedown and ratcheting. ASME does not address such methods; one might take the viewpoint that such methods fall under inelastic analysis of structures, although the argument may be considered by some to be weak. Many people will consider inelastic analysis of a structure to be the prediction of to elastic-plastic-creep deformation for a cycle by means of increments in time to obtain a time dependent solution. Limit analysis, of which limit load analysis is a subset, is not a time dependent inelastic analysis as such. While not specifically indicated, the A \& B Tests in Appendix T are limit analysis approaches, but limited in scope and application. Hence, a rank of 'NOT ADDRESED' was given.

Justification \& Documentation: ASME (Appendix T) does not provide 'justification and documentation'. 
Cycle Definition: Limited guidance is provided in Appendix $\mathrm{T}$ for definition of cycles from a load histogram. Issues with regards to 'carry-over' stresses (residual stresses) are addressed to some extent in the A-Test in Appendix T; however, the guidance is not clear and confusing. In addition, permission to use several different methods, e.g. A-1 Test and A-2 Test, for different cycles in a load histogram is not clear. As such, Appendix T of ASME NH was provided a rank of 'inadequate'.

Rapid vs. Slow Cycle: ASME actually provides solutions for both rapid cycle and slow cycle analysis of the classical 'Bree tube', i.e. constant primary load with cyclic linear secondary bending stress through the wall of an infinite tube. At elevated temperatures where the yield strength varies significantly, and where creep deformation may be significant, the difference between shakedown and ratcheting solutions, including subsequent assessment of the elastic core stress become significant between rapid and slow cycle solutions. ASME does not illustrate this to any extent, other than providing a different name to the 'Test', e.g. A-Test vs B-Test. In fact, one of the B-Tests is not a slow cycle solution, but rather a rapid cycle solution, which adds to the lack of clarity. The lack of clarity, as well as the limitation of the slow cycle solution to cases other than the classical 'Bree tube' problem limited the rank to 'EFFECTIVE'.

\subsubsection{R5 - Summary}

\section{Primary Load Limits - Time Independent:}

Elastic: R5 borrows directly from ASME's definition of primary and secondary stresses as well as peak stresses. Hence, a rank of ' 0 ' was given indicating it is the same as ASME.

Inelastic: Unlike ASME, R5 permits and encourages the use of inelastic analysis. A section is specifically includes that provides guidance on inelastic analysis and the use of various forms of constitutive equations. A rank of ' ++ ' was provided, indicating 
'effective'. A rank of 'excellent' $(+++)$ was not provided, as the direct use of inelastic analysis results to satisfy all design requirements was not always apparent.

Limit Load Method: Unlike ASME, R5 permits and to a large extent ecnourages the use of limit load methods, e.g. limit load analysis and reference stress solutions, to satisfy primary load limits. A ranking of ' +++ ' was given as the use of such approaches and incorporation with design criteria was 'excellent'.

Justification \& Documentation: R5 provides extensive and excellent references throughout each section of the Code.

\section{Primary Load Limits - Time Dependent:}

Elastic: R5 does not preclude the use of elastic analysis. Unlike ASME, R5 does not offer a means of accounting for stress redistribution due to creep deformation with elastic methods. Instead, R5 relies heavily on the use of limit load analysis and reference stress methods. The authors do not find this to be an oversight, rather a preference for use of what is often times a more appropriate approach than elastic analysis. That said, on the basis of direct comparison with ASME's elastic analysis approach, the R5 approach is 'worse' than ASME.

Inelastic: Again, R5 permits and encourages the use of inelastic analysis. The same section indicated above in inelastic analysis for primary load limits specifically includes guidance on inelastic analysis and the use of various forms of constitutive equations. A rank of ' ++ ' was provided, indicating 'effective'. Similarly, a rank of 'excellent' $(+++)$ was not provided, as the direct use of inelastic analysis results to satisfy all design requirements was not always apparent.

Limit Load Method: Limit load analysis and its application in the form of a reference stress approach is the heart of R5's approach to time dependent primary load limits. R5 encourages, to the extent that it nearly requires, the use of reference stress. A wide range 
of methods to obtain the reference stress are permitted. A ranking of ' +++ ' was given as the use of such approaches and incorporation with design criteria was 'excellent'. Note, the use of reference stress approaches requires that materials are creep ductile for the entire period of service.

Justification \& Documentation: R5 provides extensive documentation to justify the procedures and guidelines.

\section{Deformation Controlled Limits:}

Elastic Follow-Up: R5 also recognizes the importance of elastic follow-up on the behavior of structures and the subsequent importance on design criteria and analysis methods. With respect to cyclic loading, three options exist to address elastic follow-up, varying in extent of analysis and degree of conservatism. The options permit more realistic prediction of structural behavior, and likely have similar impact on assessment of strain range prediction for fatigue, creep strain increments, and creep-fatigue damage. When inelastic or reference stress approaches are used, stress classification issues are no longer an issue. The approaches lend themselves to modern FEA analysis, as well as simplified analysis. Consequently, a rank of ‘+ +' ('effective’) was given.

Elastic Analysis: R5 does permit elastic analysis, in the context of elastic stress analysis and stress classification with results applied to a rapid cycle time and temperature dependent elastic, shakedown and ratcheting analysis that is very similar to but not identical to the A-1 Test of ASME. If elastic analysis is used, limitations for applicability to general structures and secondary loads other than linear thermal bending stresses exist similar to that of ASME. As such, a rank of ' 0 ' was provided.

Inelastic Analysis: Again, R5 permits the use of inelastic analysis in the context of incremental time steps and analysis of elastic, plastic, and creep behavior of a structure. A rank of ' ++ ' was provided, indicating 'effective'. The main reason for not providing a 
rank of 'excellent' (' +++ ') is similar to that for primary load limits - the direct use of inelastic analysis to satisfy design requirements was not always apparent.

Limit Load Method: Limit load analysis is the heart of R5's use of reference stress solutions and design criteria incorporating the rupture reference stress. In terms of limit load analysis, this provides only one point or loading condition for evaluating shakedown and ratcheting conditions. For cyclic loading, a rapid cycle analysis is utilized to demonstrate shakedown, or the lack of shakedown. R5 does not limit the type of analysis that one may use to demonstrate shakedown, as a large number of tools exist to achieve this today. The cyclic state is then described by a shakedown reference stress. This shakedown reference stress is then compared against a rupture reference stress in order to satisfy design criteria against rupture and excessive strain. A rank of 'effective' ('++') was given. A few main issues were found: the shakedown reference stress is not identical to a core stress, and as such may be overly conservative relative to rupture and excessive strain accumulation. Also, R5 does not permit structures to operate in the ratcheting regime - if so, full inelastic analysis must be conducted.

Justification \& Documentation: Again, R5 provides extensive and excellent references throughout each section of the Code.

Cycle Definition: R5 does provide guidance on construction of cycles and cycle types. This in fact is a very important topic which receives limited attention or recognition of importance. The guidance provided is more than that provided by ASME; additional guidance and examples with relevance to incorporation with subsequent analysis would be very useful. A rank of '+’ ('better’) was given.

Rapid vs. Slow Cycle: R5 does not provide an option for slow cycle solutions. All solutions in R5 are based upon rapid cycle analysis. One exception does exist; the use of a time and temperature dependent yield strength as an effective yield strength is permitted; this is not a very effective slow cycle solution, but rather is a means of minimizing the shakedown reference stress and creep rupture damage by optimizing the 
residual stress field. The solution can be very conservative at very high temperatures or combinations of high temperature and long periods of time as the solution can drastically reduce the permissible operating conditions where 'shakedown' is predicted. The B-1 and B-3 Tests of ASME provide more relevant and less conservative solutions for slow cycle cyclic states of a structure, although the B-Tests are limited to the Bree tube problem. As such, R5 was determined to be 'significantly worse' ('- -') than ASME with respect to slow vs. rapid cycle solutions to predict the steady cyclic state of a structure.

\subsubsection{RCC-MR - Summary}

\section{Primary Load Limits - Time Independent:}

Elastic: RCC-MR classifies primary, secondary and peak stresses in the same manner as ASME/NH does. A rank of ' 0 ' was given indicating it is the same as ASME.

Inelastic: RCC-MR permits the use of inelastic analysis, unlike ASME. It provides appendices that describe inelastic analysis methods and constitutive material models to be used. A rank of ' ++ ' was provided.

Limit Load Method: While ASME does not allow it, RCC-MR permits limit load methods for primary loads. A ranking of ' ++ ', 'effective' was given; this is short of 'EXCELLENT' since the code did not describe and treat the method and its application to general loading and general components with any significant detail.

Justification \& Documentation: RCC-MR sections on failure mechanisms and design criteria include brief documentation of the procedure but no references or examples. For that, RCC-MR was given the rank of ' + '. The relevant appendices were not available, and they might include references and documentations, but it was not obvious whether that was the case or not. 


\section{Primary Load Limits - Time Dependent:}

Elastic: RCC-MR includes the use of elastic analysis. Like ASME, RCC-MR provides a means of accounting for stress redistribution due to creep deformation with elastic methods. Therefore, RCC-MR is ranked equal '0’ with respect to ASME.

Inelastic: RCC-MR permits the use of inelastic analysis, while ASME does not. A rank of 'effective’ $(++)$ was provided, because the use of inelastic analysis to address all design requirements was not clear.

Limit Load Method: Limit load analysis is allowed in RCC-MR and may not be mixed with other methods for time dependent primary load limits; ASME doe not allow this method. A ranking of ' ++ ' was given. A rank of 'excellent' was not given since the approach was not treated to any great extent; it was only mentioned that limit load method may be used, and the corresponding design limits were provided.

Justification \& Documentation: RCC-MR includes brief explanation of the design procedures but does not include references, examples or justifications. For that, RCCMR was given the rank of ' 0 '. The relevant appendices, which might include documentations and examples, were not available to the authors. However it was not obvious whether that was the case or not.

\section{Deformation Controlled Limits:}

Elastic Follow-Up: RCC-MR recognizes the need to address elastic follow-up in the analysis methods, but it does not treat this issue adequately; for the most part, the code simply has a general disclaimer that the elastic follow-up needs to be addressed, and in certain design criteria it assumes a very conservative elastic follow-up factor of 3 , and gives the analyst the choice of reducing this factor if he can prove the adequacy of the

lower value; it does not provide any guidelines for obtaining a more realistic follow-up 
factor, and it does not provide any guidelines for checking the significance of elastic follow-up. The rank of ' 0 ' was given to RCC-MR.

Elastic Analysis: RCC-MR has an extensive elastic analysis procedure. However, the procedure seems to be limited to the Bree tube type problem and its applicability to general structures and more complex loading is not addressed. While the elastic analysis procedure is quite extensive (requiring the definition of efficiency index and secondary ratios), it is difficult to assess whether it is better or worse than that of ASME. Therefore, a rank of ' 0 ' was given.

Inelastic Analysis: RCC-MR permits the use of inelastic analysis elasto-plastic, and visco-elasto-plastic types. A rank of '+ +' was provided, indicating 'significantly better', because RCC-MR seems to devote more effort to and provides some direction for inelastic analysis. The availability of relevant appendices would have shed more light on this issue. The reason a rank of 'excellent' (' +++ ') was not provided is the lack of direction of how inelastic analysis results would be used satisfy certain design criteria.

Limit Load Method: Limit load analysis is not permitted in RCC-MR, like ASME.

Justification \& Documentation: RCC-MR does a good job describing in detail the elastic procedure, and it provides appendices for describing the inelastic analysis, including constitutive behavior. However, there is no evidence that the code provides references, examples or justification, and that is why the ranking was not higher than ' + '.

Cycle Definition: RCC-MR devotes significant effort to defining cycles (RB3263). In fact it has a section on cycle definition. However, the description is somewhat ambiguous, possibly due to translation difficulties, and it lacks details that are critical to a complex loading scheme. None the less, the guidance provided is more than that provided by ASME; examples of some nontrivial loading cases would have been beneficial in supporting the guidelines. A rank of '+' ('better') was given. 
Rapid vs. Slow Cycle: RCC-MR does not provide a clear option for dealing with slow cycle solutions. However, it provides creep correction factors that minimize the effective stress used to predict creep strain. The procedure for obtaining the creep correction factor is included in the code but not explained or rationalized. RCC-MR is given a rank of ' 0 ' for the treatment of slow cycle.

\subsubsection{MONJU - Summary}

\section{Primary Load Limits - Time Independent:}

Elastic: MONJU is virtually identical to ASME's definition of primary and secondary stresses as well as peak stresses. The only difference is that it breaks out sort and long term stresses in the design criteria, which reflects the Japanese special concerns with seismic loading. Hence, a rank of ' 0 ' was given indicating it is the same as ASME.

Inelastic: MONJU neither permits nor prohibits inelastic analysis and, by default is identical to $\mathrm{NH}$ in this respect. However subsequent developments under the auspices of JSME appear to go much further in not only permitting inelastic analysis, but in basing a new "design without stress classification" procedure on inelastic methods. A rank of '+ +' was provided, indicating 'effective'. A rank of 'excellent' $(+++)$ was not provided, because details of the method of implementation of inelastic analysis in the new code were not available to review.

Limit Load Method: No information could be obtained to verify the use or otherwise of limit analysis in its own right, but the use of the Reference stress method implies that it may be an accepted method of analysis. The prospect of its being permitted, but with no direct evidence of if or how it is used, earns a ranking of ' +++ '.

Justification \& Documentation: Documentation on both MONJU and on more recent developments is difficult to locate. 


\section{Primary Load Limits - Time Dependent:}

Elastic: By default, this is the only approach available in the MONJU procedure. The implementation appears to be essentially the same as ASME/NH.

Inelastic: The new JSME sponsored code appears to be strongly based on inelastic analysis. However, no details could be found. Therefore it is not possible to comment on the merits of the methods used.

Limit Load Method: The use of a Reference Stress based so-called “design without stress classification” appears to be central to the new JSME sponsored code, which means that limit load analysis is used. As before, no details are available.

Justification \& Documentation: No documentation either about the code or how it is used is available

\section{Deformation Controlled Limits:}

Elastic Follow-Up: MONJU refers frequently to elastic follow-up and attempts to incorporate the concept more than any other code. In particular it provides guidelines on how to judge the extent of elastic follow-up, particularly where piping systems are concerned, and uses the concept to partition total stresses into primary and secondary components. The method offered is not entirely satisfactory, since it relies to some extent on subjective judgment in performing the partition.

Elastic Analysis: MONJU has one simplified method for dealing with cyclic loading and associated deformation limits. This is a Bree-type analysis similar to the NH B-1 test. For more complex geometries, there appears to be a procedure within the "classification-free" methodology for dealing with cyclic behavior, which limits stress ranges exceeding the yield range to an area less than $10 \%$ of the cross section. No details are available at the time of writing 
Inelastic Analysis: No detailed information is available. It can be inferred that inelastic cyclic behavior is dealt with by direct, detailed FE analysis.

\section{Limit Load Method: Not applicable}

Justification \& Documentation: There is little or no documentation about the code or the methods used.

Cycle Definition: The only specific reference to cycle definition is in the context of fatigue damage caused by seismic events. Otherwise no mention is made to cycle definition.

Rapid vs. Slow Cycle: This distinction is an artifact of deliberate attempts to simplify the problem of ratcheting analysis. While no information can be found to support this conjecture, it is believed that cyclic analysis by the new JSPME sponsored code uses detailed cyclic analysis (elastic-plastic-creep) and therefore dispenses with the concepts of rapid- and slow-cycling behavior.

\subsubsection{API579 - Summary}

API 579 is not a design code but a guideline for performance of Fitness-for-Service (FFS) assessments. As such it has different objectives from those of a design code in that it is concerned with predicting the remaining life of a component which already exists and which, furthermore, may have deviations from its original design intent in the form of defects or damage, whereas design is concerned with a notional components whose dimensions and material have yet to be determined. This difference means that FFS has less need than does design for simplified, scoping types of assessment procedures capable of evaluating a range of component options, because that decision has been made, but it has the advantage of being able to draw on more factual information on the component and its operating conditions to utilize more detailed methods of analysis such as advanced FE techniques as routine procedures. 
In the specific case of elevated temperature design, the primary design criterion is creep rupture, which is the end product of a form of cumulative damage or defectiveness. This is unusual in ASME design doctrine, which does not normally admit to the presence of defects as part of the design process but it is unavoidable in this instance. Here, the FFS approach may have special value since it is set up from the outset with the assessment of damage and defectiveness in mind.

In fact, since one way of viewing design is as a FFS assessment of a virgin, non-defective component, the tools needed for both activities are very similar, and sometimes identical in some cases. Many of the procedures and information provided in API 579 therefore have potential applications in improving code practices in the future.

In addition to having somewhat different objectives from a design code, API 579 also has a different format which does not match one-to-one with, say, Section III/NH but, within these differences, parallel activities can be identified. For instance, API 579 is constructed in three levels of assessment.

- Level 1 - A scoping level aimed at determining whether creep is a significant problem or not. This mirrors the need in a design code for a criterion for the threshold of "negligible creep". The unique feature of this level of API 579 is that it uses context specific data to determine the need for creep assessment on a caseby-case basis. Level 1 includes simplified "strength-of-materials" type calculations similar to, and in some cases identical to, the analytical methods provided in Sections I and VIII, Div. 1 of the ASME Code.

- Level 2 - In effect a rerun of original design computations but with actual dimensions, material properties and operating conditions in place of assumed, generally conservative assumed values used in design. API 579 permits the full range of computational techniques available, from elementary hand calculations, through simplified elastic FEA to detailed, inelastic and nonlinear FEA - but restricted to components with no perceivable damage. This level is therefore equivalent to the validation procedure in a design code. The specific methods offered are essentially the same as those mandated in Sections VIII, Division 2 and Section III of the ASME Design Code. 
- $\quad$ Level 3 - The same range of assessment procedures as used in a Level 2 assessment, with the additional feature of a wide spectrum of damage mechanisms and geometric defects included in the component description.

API 579 also possesses a number of other attributes which have no parallel in design codes and in Section III/NH in particular.

1. As a FFS guideline, API 579 is built round the assumption of cyclic loading for which it assumes direct cyclic FEA to be the standard approach. With the exception of low temperature time independent assessment therefore, it makes no reference to simplified procedures such as elastic follow-up or "Bree-type" diagrams for evaluation of incremental collapse. These simplified methods are therefore not so much omitted but superseded in API 579.

2. In addition to "design limits" similar to those mandated in code based design, API 579 also introduces "service limits” which are, in effect, deformation (i.e. displacement) limits on component distortion, to be specified by the owneroperator and based on functional limitations of the equipment.

3. The "service limits" described above, together with a material specific local strain limitation based on the multiaxial stress state, replace the mixed “deformation/strain” limits of 1/2/5\% membrane/bending/local strain limits prescribed in Section III/NH. Material data for a practical range of materials are provided as part of API 579 for this purpose.

4. API 579 provides a comprehensive material database covering a range of materials of practical interest in sufficient detail to permit the implementation of the detailed component analyses advocated. This database includes the MPC Omega creep constitutive model, monotonic and cyclic stress/strain curves and data needed to perform fatigue creep and combined creep/fatigue growth studies.

5. The API 579 philosophy is to be inclusive in its approach to assessment techniques. It does not constrain the user to specific methods for specific problems. On the contrary, in addition to providing recommended practices where these exist and have proven pedigrees, it encourages the user to seek other 
methods and points to other international codes and standards including the British R5 and BS7910, as well as ASME's own NH, as acceptable source of alternative methods of analysis. Its only caveat is that any method employed be validated and documented so that the results can be reproduced by an independent agent.

6. API 579 places great store on documentation. This is not limited to record keeping on individual projects, but on its own methodologies, the latter being supported by an extensive bibliography of state-of-the art developments in FFS technology.

7. In existing codes there is a mismatch in creep rupture assessment, in that simple "strength-of-materials" methods used successfully for many years are based on primary stresses, and ignore local peaks, or even creep relaxed local peaks, whereas more recent “advanced” methods invariably base component failure on local criteria with or without allowance for creep relaxation. In highly complex geometries this leads to significant conservatism. A more realistic assessment of creep damage should include some measure of damage propagation following initiation. In local strain concentrations this would take the form of a creep crack propagation analysis. API 579 contains a methodology for carrying out this extension of time-to-failure by including creep crack growth using a simplified method that could be adapted for design purposes.

To the extent that it is possible, the following sections attempt to compare API 579 methodology with that of existing elevated temperature design practice.

\section{Primary Load Limits - Time Independent:}

Elastic: Provided as one of several options. To all practical intents, API 579 practice follows that of the ASME Code in providing a simplified linear elastic FE analysis based on stress classification. It goes further than the Code in offering several practical methods of implementing stress linearization with special reference to complex 3-dimensional geometries. 
Inelastic: API 579 permits and encourages the use of inelastic analysis as an alternative to elastic. A rank of 'excellent' $(+++)$ is provided, as the direct use of inelastic analysis results to satisfy all design requirements is spelt out in detail.

Limit Load Method: API 579 uses limit load concepts in several contexts, including one intermediate level of simplified inelastic analysis. It provides a compendium of standard solutions for common geometries. It gives guidance on the use of limit load analysis in cyclic loading and in analysis of situations where collapse due to combined mechanical loading occurs, short of a full inelastic, cyclic analysis. A ranking of ' +' was given as the use of such approaches and incorporation with design criteria was 'adequate'.

Justification \& Documentation: API, like R5, provides extensive and excellent references throughout each section of the Code.

\section{Primary Load Limits - Time Dependent:}

Elastic: API 579 permits essentially the same elastic based method of analysis mandated in Section III/NH to be applied to primary load assessment in the creep range. In fact, due to the bipartisan nature of the task group developing API 579, much of the ASME Code methodology has been adopted unchanged. No allowance is made in API 579 for creep induced stress redistribution as is attempted in $\mathrm{NH}$. However, the latter is so marginal that the difference is insignificant.

Inelastic: API 579 relies heavily on FE based inelastic analysis, especially at Level 3. Within this category, there are sublevels of detail, including "simplified" inelastic methods using either linear analysis as a baseline, or limit analysis using elastic/perfectly plastic idealizations of material, but it includes the prospect of fully detailed nonlinear material, nonlinear geometry FEA, for the purposes of which it provides extensive material property data as well as step-by-step guidance on how to implement the analysis and interpret the results in term of damage and remaining life. A ranking of ' +++ ' is provided. Creep failure, whether under monotonic or cyclic conditions can be computed 
with the best accuracy available within the current state of the art, using inelastic analysis combined with the Omega creep model. This approach encompasses both "creep ductile" behavior for which the Reference Stress approach is an acceptable approximation and for "creep brittle” materials, such as weld interfaces, where creep ductility is not an acceptable assumption.

Limit Load Method: Limit load analysis and its application in the form of a reference stress approach are both used and referred to in passing but no great emphasis is placed on the use of the Reference Stress concept - in the context of creep analysis. For this purpose, API 579 prefers to use either a very simplified "strength-of-materials" approach, as is employed in Sections I and VIII of the ASME Code (which are, in effect "reference stress calculations without being recognized as such) or the local damage based methodology implemented in $\mathrm{NH}$ based on elastic analysis but extended to include inelastic material behavior. This approach is inherently conservative because redundant structures do not fail when the critical conditions are reached at a point, but only after some redistribution of damage has occurred. The Reference Stress technique as implemented by R5 does capture this phenomenon as does, ironically the simple "strength-of-materials" approach.

Justification \& Documentation: API, like R5, provides extensive and excellent references throughout each section of the Code.

\section{Deformation Controlled Limits:}

Elastic Follow-Up: This concept is not recognized in API 579, being replaced with direct cyclic analysis, including creep relaxation. This is not an omission but a displacement by more advanced methodology.

Elastic Analysis: In the context of deformation limits API 579 departs from the template of the other codes and standards reviewed in this report in that it distinguishes explicitly between displacement limits, which are user defined based on function, and local strain 
limits, which, instead of being constrained to arbitrary limits of 1, 2 and 5\%, are limited by listed material specific ductility, corrected for multiaxial stress state.

Inelastic Analysis: This is the preferred method advocated by API 579 for assessment of cumulative deformation and local strains. As for "elastic" analysis, limits are separated into structural deformations, and local strains and case or material specific limits are applied separately to each. Guidance and documentation are both excellent and earn a rating of ' +++ '

Limit Load Method: Shakedown and ratcheting are both evaluated directly in API 579, so that it finds no need for limit load based procedures. This category does not apply.

Justification \& Documentation: API, like R5, provides extensive and excellent references throughout each section of the Code.

Cycle Definition: API 579 gives considerable time and effort to the definition of load and thermal cycles. This is a corner stone of the document and rates ' +++ '.

Rapid vs. Slow Cycle: By the nature of the approach taken by API 579 to cyclic loading, the concepts of "slow" and "rapid" cycle have no relevance in its assessment methodology. 


\subsubsection{ASME NH}

ASME NH [1] is the basis for which many or all of the International Codes, elevated temperature and non-elevated temperature design, are based. The concept of primary and secondary stresses, as well as membrane, bending, peak, and local membrane stresses were instrumental, serving as the basis for current design by analysis and design by rule approaches in ASME. Application of such concepts is straightforward at temperatures where creep is deemed insignificant. The challenge lies in applying such concepts when creep mechanisms become significant.

Later in this report, the differences between International ETD Codes lies primarily in how approaches have evolved to incorporate creep behavior and related failure mechanisms while maintaining the basic foundation from which the ASME Code was built - stress classification and stress linearization. Advances in computational power over the last two decades has permitted more complex geometries and loadings to be evaluated than ever before, particularly in terms of full inelastic analysis (elastic viscoplastic analysis). Still, the design of structures is constrained by economics (cost), resulting in the desire to use simplified design methods to reduce, minimize, or eliminate the need for full inelastic analysis. The relevance to industrial needs has played a significant role in terms of what types of design methods have been developed, and implemented; for example, Liquid Metal Fast Breeder Reactors (LMFBR) were the driving force behind much of the activity in ASME NH development in the 1970's, with subsequent efforts in the 1980-1990s due to High Temperature Gas Reactors (HTGR), and recently the Next Generation Nuclear Plant, also an HTGR concept.

The demise of the nuclear industry in the United States resulted in relatively small activities in high temperature structural design efforts within the United States, and in ASME NH. However, strong programs in Japan, France, and the United Kingdom continued. The differences between the International Codes reviewed herein are largely due to such circumstances. 


\subsubsection{PRIMARY LOAD LIMITS}

Elastic analysis is addressed with respect to satisfying primary load limits and secondary (cyclic) load limits. Prior to conducting elastic analysis, loads must be categorized as primary or secondary; this step is aided by use of Tables NH-3217-1; subsequent application of design rules require stress linearization. Stress linearization continues to be a topic of debate, the details of which are beyond the scope of this report; however, this debate is partially responsible for the development of alternative design methods in International Codes and in the literature.

\subsection{Elastic Analysis \& Limit Load Analysis}

Elastic and limit load analysis are addressed in the same section due to the nature by which ASME evolved. Reference stress methods for primary load limits are very similar to limit analysis, but are addressed separately. This section is organized according to a) the type of stress analysis, and b) the design criteria that incorporates the stress intensity and allowable stress to address specific failure mechanisms.

\section{Stress Analysis:}

Prior to finite element codes, elastic analysis was conducted by application of solid mechanics, e.g. theory of plates and shells. This requires execution of engineering fundamentals, including simplification of the geometry and loading and construction of adequate but representative free body diagrams. Assumptions of how loads may be transferred from one portion of the structure to another are often required, e.g. on free body diagram to another. These approaches, while quite acceptable and necessary from an engineering standpoint, can often result in different but acceptable predictions of primary and secondary stresses. One example is the analysis of a pressure vessel with a flat end. Finite element analysis simplifies this analysis to a great extent, but still requires proper interpretation. Debate continues as to how to universally linearize stresses from finite element analysis. 
ASME NH does not permit inelastic analysis to satisfy primary load limits (NH-3220), with the exception of Level D Service Loads, where full inelastic analysis may be used rather than elastic (NH-3225). Consequently, stress levels predicted with elastic analysis will result in more conservative designs (higher stresses) than if creep were taken into account. However, ASME NH does recognize this, accounting for stress relaxation at the fibers of a vessel wall subjected to primary bending stresses; this is accomplished with a factor ' $K_{t}$ ', which is a function of the section factor ' $K$ '. $K$, and consequently $K_{t}$, are a function of the cross-section being considered (NH 3223, Table A-9521(b)-1 Appendix A Section III).

ASME's restriction to elastic analysis has influenced the various design criteria, since each design criterion must compare the stresses to some permissible strength parameter to address one or more failure mechanisms. The methods utilized to determine the permissible strength parameters, e.g. rupture stress, are outside of the scope of this report. However, the concepts supporting the design criterion (stress vs. strength) are discussed. Such discussions will be particularly important in later sections, as alternative approaches to those permitted by ASME NH.

\section{Failure Mechanisms \& Design Criteria:}

Strictly speaking, limit load analysis is not directly permitted in ASME NH. Rather, the use of elastic analysis is coupled with a safety factor to achieve the same or more conservative result. This approach, while perhaps not intentional, is directly supported by work of Langer [2]. As discussed in [3], limit analysis is the basis for low temperature service where creep is considered insignificant; ASME Section III Subsection NB 3228 “Applications of Plastic Analysis" permits the use of "limit analysis" or "plastic analysis" to satisfy a structures ability to resist primary loading for time-independent failure. Similarly, so does Section VIII Div2 Mandatory Appendix 4 (4-136.3 Limit Analysis). In fact, ASME Section I, Section III, and Section VIII Div 2 are based upon limit analysis as discussed by Langer, "The choice of the basic stress intensity limits for stress categories....was accomplished by the application of limit design theory tempered by engineering judgment and some conservative simplifications” [2]. 
With this knowledge in mind, let us review elastic analysis as it pertains to failure mechanisms addressed by Primary Load Limits in ASME NH. McGreevy has provided a review in earlier efforts for NGNP, including interpretation and comparison with reference stress approaches [3]. A brief summary of the same is provided below.

In NH-3220, the following criteria apply, and are adjusted slightly depending upon the Service Load Level (A\&B, C, or D) by safety factors. Hence, only the Level A \& B Service Limits are summarized. Furthermore, it should be noted, that ASME NH utilizes the average temperature through the thickness when satisfying the following design criteria.

- $\quad$ Limit load collapse under a single load application for time independent failure: primary membrane loading only The primary membrane stress cannot exceed the time independent strength: $\mathrm{NH}$ 3220, Eqn (3): $\mathrm{P}_{\mathrm{m}}<\mathrm{S}_{\mathrm{mt}}$. Here, no adjustment for $\mathrm{K}_{\mathrm{t}}$ or interaction of membrane and bending is required; $\mathrm{S}_{\mathrm{mt}}$ addresses both time dependent and time independent failure. $S_{m}$ is clearly a subset of $S_{m t}$, with $S_{m t}$ being more conservative than $S_{m}$.

- $\quad$ Limit load collapse under a single load application for time independent failure: primary membrane and bending The primary membrane stress (including any local effects on membrane stress) cannot exceed the time independent strength: $\mathrm{NH}$ 3220, Eqn (4): $\mathrm{P}_{\mathrm{L}}+\mathrm{P}_{\mathrm{b}}<\mathrm{KS}_{\mathrm{m}}$. Here, $\mathrm{K}$ is 1.5 for typical across the wall bending of shell structures (rectangular sections) and was achieved based upon limit load analysis.

- Deformation limit for time independent failure: primary membrane and/or primary bending No criteria exist; one must provide and satisfy criteria in the Design Specification (NCA-3250), and the manufacturer must supply criteria to the Owner.

- Deformation limit for time dependent failure: primary membrane only 
The primary membrane stress cannot exceed the time dependent strength: $\mathrm{NH}$ 3220, Eqn (3): $\mathrm{P}_{\mathrm{m}}<\mathrm{S}_{\mathrm{mt}}$. Here, no adjustment for $\mathrm{K}_{\mathrm{t}}$ or interaction of membrane and bending is required; $\mathrm{S}_{\mathrm{mt}}$ includes a limit on time to $1 \%$ total strain, or rupture, whichever is lower. Stricter limits may be required, depending upon the design and are left to the designer/manufacturer to specify and supply criteria to the Owner.

- Excessive creep deformation and rupture limit for time dependent failure: primary membrane only

The primary membrane stress cannot exceed the time dependent strength: $\mathrm{NH}$ 3220, Eqn (3): $P_{m}<S_{m t}$. Here, no adjustment for $K_{t}$ or interaction of membrane and bending is required; $S_{\mathrm{mt}}$ includes a limit relative to minimum creep rupture, onset of tertiary creep, and time to $1 \%$ total strain, whichever is lowest.

- Excessive creep deformation and rupture limit for time dependent failure: primary membrane and bending

The primary membrane and bending stress cannot exceed the time dependent strength: NH 3220, Eqn (5): $P_{L}+P_{b} / K_{t}<S_{t}$. Here, the adjustment for creep at the outer fibers is made via use of $K_{t}$. $S_{t}$ is equal to or less than $S_{m t}$, where $S_{t}$ includes a limit relative to minimum creep rupture, onset of tertiary creep, and time to $1 \%$ total strain, whichever is lowest.

* For cases where the time is less than the total service life, the use-fraction sum associated with time dependent primary limits must be satisfied: $\sum_{i}\left(\frac{t_{i}}{t_{i r}}\right) \leq B_{r}$.

- Non-ductile fracture:

ASME restricts materials, duration of use, and time with the intent to avoid any failure by nonductile fracture while at elevated temperatures (NH-3241). However, creep relaxation may lead to high residual stresses during cycles at the lowest temperatures of service. In such cases, if creep is insignificant, Appendix $\mathrm{G}$ of Section III is an acceptable procedure for preventing nonductile fracture for ferritic materials. Otherwise, additional measures are required, but not specified. For austenitics, (Type 304 SS, Type 316 SS, or Alloy $800 H$ ), such justification is 
not required unless fabrication effects alter the fracture characteristics of the material. Currently, there are no other Code approved non-ferritic or nonaustenitic materials, such as Alloy 617, although a Draft Code Case exists for the material.

- Failures of Weldments:

Time dependent effects of weldments are addressed by modification of allowable stresses (NH-3221) by definition of St, where St $=\min$ (St in Tables I-14.4, $0.8 * \mathrm{~S}_{\mathrm{r}} * \mathrm{R}$ ), where $\mathrm{R}$ is the ratio of weld metal creep rupture strength to base metal creep rupture strength (note, this factor apparently only addresses rupture, not time to $1 \%$ strain or onset of tertiary creep).

- Multiaxial effects on Creep Failure:

No criteria address this concern (deformation or rupture effects) for Primary Limits, except for the limitation that the algebraic sum of the three primary stresses shall not exceed four times the tabulated value of $S_{\mathrm{mt}}(\mathrm{NH}-3227.4)$.

\subsection{Reference Stress Methods}

ASME does not incorporate reference stress methods; depending upon the method, the term 'reference stress' may refer to different stress conditions, values, and have different intentions and application. . 


\subsubsection{DEFORMATION CONTROLLED LIMITS}

Deformation controlled limits address failure mechanisms that result from secondary stresses, and peak stresses. One must specify the appropriate approaches and justification in the Design Specification (NCA-3250) per NH-3252 to address associated failure mechanisms. NH-3651 requires use of App. T for piping; otherwise, Appendix T is NonMandatory, and an alternative criteria proposed by the manufacturer is permissible.

Unless specified otherwise, reference to Deformation Controlled Limits in ASME are actually found in Appendix T. The main sections are T-1300 (strain limits, including ratcheting), T-1400 (creep-fatigue), T-1500 (buckling \& instability limits), and T-1700 (special requirements, e.g. strain requirements at weldments).

Of greatest relevance for this review, elastic and simplified inelastic analyses are utilized to address strain limits including shakedown and ratcheting analysis in (T-1300), and C-F (T-1400). Appendix T is summarized with respect to elastic and simplified inelastic analysis herein.

C-F analysis is out of scope; however, where appropriate, relevance of simplified inelastic (or elastic) analysis to C-F is noted. No reference stress methods are used in Appendix T, nor are limit load analysis with the only possible exception of the requirement of the use of a unified constitutive equation for Mod9Cr1Mo in buckling and stability analysis of T-1520. Note, while Appendix T does not utilize such approaches, ASME NH permits alternative approaches that may include limit load, reference stress, and other approaches; such approaches must be specified in the Design Specification (NCA-3250). Note, ASME does not call out or specify such alternative approach.

Prior to launching into specific Code comparisons, a brief comment is required with regards to various types of ratcheting analysis. The intent of a majority of shakedown and ratcheting analysis is conducted with the intent of determining the steady cyclic state of the structure. Typically this is accomplished with a variety of simplified elastic-plastic analysis methods, otherwise, a full and detailed inelastic analysis would be required. 
Among the steady cyclic state solutions that result from various techniques are the rapid cycle analyses, slow cycle analyses, and variations in-between $[4,5]$.

Rapid cycle analysis is an elastic-plastic analysis of a structure subjected to a repeated cyclic load histogram; creep is not permitted to occur, i.e. the rate of loading does not permit creep to occur, or the analysis is conducted without creep. Shakedown and ratcheting theories provide proof that this is the most conservative treatment of the steady cyclic state predicted with rapid cycle analysis.

If creep is permitted to take place, and the actual duration of the load histogram elapses, then portions of the structure will experience stress redistribution due to creep deformation. Eventually, the structure reaches a steady cyclic state where elastic, plastic, and creep deformation occur, but where the state of the structure at time ' $\mathrm{t}$ ' and ' $\mathrm{t}+\Delta \mathrm{T}$ ' from one cycle to the next are identical, where $\Delta \mathrm{T}$ is the period of the cycle.

Several variations of rapid cycle analysis and slow cycle analysis have been proposed and/or are utilized by Codes. These include use of rapid cycle analysis with time and temperature dependent yield strengths rather than temperature dependent yield strengths. Furthermore, a variant of the slow cycle solution exists as well; in such a case, the stress distribution at the start of a dwell period is determined based upon beneficial residual stresses resulting from full creep relaxation of secondary stresses during the dwell period. This variant of the slow cycle solution, along with other restrictions on its use, provide an upper bound on the slow cycle solution, and hence, a less conservative bound on the steady cyclic state than rapid cycle analysis.

\subsection{Elastic Analysis}

Strain limits are required, including limitations on strains according to the following values:

- strains averaged through the thickness, $1 \%$ 
- strains at the surface, due to an equivalent linear distribution of strain through the thickness, $2 \%$

- local strains at any point, $5 \%$

Similar to the Primary Load Limits, temperature is the average temperature through the thickness.

McGreevy recently provided a review of the A-Tests [6]; there are three - A-1, A-2, and A-3. All of the A-Tests are based upon the classical Bree tube problem: an infinitely long tube, subjected to a constant primary membrane stress and a cyclic through the wall linear thermal gradient. If and where applicable, any suggested modifications of ASME are reserved until Subtask 9.5; however, weaknesses and strengths are summarized herein.

Note, the A-Tests require elastic stress analysis (stress categorization and linearization) and address deformation (strain ) failure mechanisms or limits only, with the exception of the A-3 Test, which addresses creep rupture damage and deformation limits in terms of satisfying several insignificant creep criteria.

\section{A-1 Test:}

While one intent is to limit the average creep strain across a section to less than $1 \%$, the constraint is not enforced directly by any of the A-Tests; satisfying one of the A-Tests alone is insufficient to enforce the $1 \%$ limit. Rather, the A-Tests serve as means to demonstrate that no plastic ratchet strain and no enhanced creep strain occurs as a result of the combination of applied loads and residual stresses, with residual stresses resulting from creep relaxation of secondary stresses during high temperature service. The $1 \%$ strain limit is enforced with the additional requirement of satisfying primary load limits of NH-3000.

The current A-1 Test has the following key aspects:

1. The method utilizes elastic stresses obtained after stress categorization and linearization. $\mathrm{X}$ is the normalized primary stress intensity parameter, where 
$\mathrm{X}=\left(\mathrm{P}_{\mathrm{L}}+\mathrm{P}_{\mathrm{b}} / \mathrm{K}_{\mathrm{t}}\right) / \mathrm{S}_{\mathrm{y}}$ and $\mathrm{Y}$ is the normalized secondary stress intensity parameter, where $\mathrm{Y}=\left(\mathrm{Q}_{\mathrm{R}}\right)_{\max } / \mathrm{S}_{\mathrm{y}} . \mathrm{K}_{\mathrm{t}}=(\mathrm{K}+1) / 2$, where $\mathrm{K}$ is a section factor that is the ratio of the load set producing a fully plastic section to a load set producing initial yielding of the extreme fiber of the cross section, see Appendix A of Sec III, Table A-9521(b)-1.

2. The method does impose a cycle definition restriction for $\mathrm{X}$ and $\mathrm{Y}$. At least one cycle must be defined that includes the maximum secondary stress intensity range, $\mathrm{Q}_{\mathrm{R}}$, and the maximum value of $\left(\mathrm{P}_{\mathrm{L}}+\mathrm{P}_{\mathrm{b}} / \mathrm{K}_{\mathrm{t}}\right)$, which occur during all Level A, B, and C Service Loadings. This means that the combination of the maximum $\mathrm{Q}_{\mathrm{R}}$ and the maximum value of $\left(\mathrm{P}_{\mathrm{L}}+\mathrm{P}_{\mathrm{b}} / \mathrm{K}_{\mathrm{t}}\right)$ serve to define the worst possible loading case, even if they do not occur in the same cycle in the load histogram (with the exception of any Level D Service Loads).

3. The method includes no temperature restrictions, e.g. it does not require one end of the cycle to be below the creep range.

4. The method has no time restriction on individual cycles or the entire load history.

5. The yield strength used to normalize $S_{y}$ is defined as the average of $S_{y}$ values at the maximum and minimum wall averaged temperatures during the cycle. i.e. $\mathrm{S}_{\mathrm{y}}=$ average $\left(\mathrm{S}_{\mathrm{yL}}, \mathrm{S}_{\mathrm{yH}}\right)$. In fact, after examination, it is clear that the choice of how to normalize primary and secondary stresses to obtain $\mathrm{X}$ and $\mathrm{Y}$, respectively, is irrelevant (i.e. it has no impact on the solution).

6. The governing equation, Eqn (1) I T-1300 is given as: $X+Y<=S_{a} / S_{y}$ where $S_{a}$ is an effective yield strength that is both temperature and time dependent $\rightarrow \mathrm{S}_{\mathrm{a}}=\min \left(\mathrm{S}_{\mathrm{y}}, 1.25 \mathrm{~S}_{\mathrm{t}} @ \mathrm{~T}_{\mathrm{H}} @ 10 \mathrm{khrs}\right)$ where $\mathrm{T}_{\mathrm{H}}$ is the core temperature at the hot end of the cycle in question (not the maximum temperature of the load history).

Eqn (1) can be rewritten as: $\mathrm{P} / \mathrm{S}_{\mathrm{y}}+\mathrm{Q}_{\mathrm{R}} / \mathrm{S}_{\mathrm{y}}<=\mathrm{S}_{\mathrm{a}} / \mathrm{S}_{\mathrm{y}}$. Multiplying by $\mathrm{S}_{\mathrm{y}}$ reveals: $\mathrm{P}+\mathrm{Q}_{\mathrm{R}}<=\mathrm{S}_{\mathrm{a}}$. Note, the equation is independent of $\mathrm{S}_{\mathrm{y}}$. 
The A-1 Test requires that the sum of the primary and maximum secondary stress range be less than the strength parameter, $S_{a}$. $S_{a}$ is an effective yield strength, and hence, the A1 Test requires that elastically calculated stresses must not exceed the effective yield strength $S_{a}$.

Several weaknesses are observed [6].

1. Utilizing the maximum $\mathrm{Q}_{\mathrm{R}}$ and the maximum value of $\left(\mathrm{P}_{\mathrm{L}}+\mathrm{P}_{\mathrm{b}} / \mathrm{K}_{\mathrm{t}}\right)$ does not necessarily define the maximum values of $\mathrm{X}$ and $\mathrm{Y}$. Why, because it is not necessarily true that the maximum core temperature, and hence the effective yield strength ( $\mathrm{S}_{\mathrm{y}}$, as defined in $\left.\mathrm{T}-1321\right)$ corresponds to maximum values of $\mathrm{X}$ and $\mathrm{Y}$. Recall, the intent to combine the maximum $\mathrm{Q}_{\mathrm{R}}$ and maximum $\left(\mathrm{P}_{\mathrm{L}}+\mathrm{P}_{\mathrm{b}} / \mathrm{K}_{\mathrm{t}}\right)$ are not impacted by the value of $S_{y}$. Since $S_{a}$ is a function of the core temperature, the core temperature corresponding to maximum $\mathrm{Q}_{\mathrm{R}}$ and maximum $\left(\mathrm{P}_{\mathrm{L}}+\mathrm{P}_{\mathrm{b}} / \mathrm{K}_{\mathrm{t}}\right)$ is crucial.

2. Appendix $\mathrm{T}$ is not clear as to whether or not the A-Tests may be used in combination with one another to address various load cycles during the lifetime of the component or structure.

3. The A-1 Test is not clear as to whether the cycle definition is restricted to the 'whole life' or not.

4. The use of an effective yield strength, $S_{a}$, which is dependent upon $S_{t}$ at a time of 10,000 hours is overly conservative. The A- 1 Test is a rapid cycle solution, which conservatively ensures that no yielding occurs; as such, the Primary Load limits ensure against the $1 \%$ strain limit. Implementing a time dependent yield strength is not required.

5. The A-1 Test does not refer to NH-3000 subsequently serving to satisfy the $1 \%$ strain limit.

6. The effects of cyclic softening or hardening on yield strength are not considered. In terms of hardening, the A-1 Test will be conservative, whereas in the case of cyclically softening materials, the A-1 Test will be unconservative.

The strengths of the A-1 Test are: 
1. The test is a simple fast screening process that may serve to justify no enhanced creep and no plastic ratcheting strains for many components.

2. There are no temperature or time restrictions on the load histogram for the test.

\section{A-2 Test:}

The A-2 Test includes identical definitions to X and Y. Key aspects of the method or test are:

1. The method includes the requirement of a cold-end at one extreme of cycle according to Table T-1323.

2. The method has no time restriction for core stresses.

3. The method does impose the cycle definition restriction in terms of $X$ and $Y$ discussed earlier for the A-1 Test.

4. In summary: the $\mathrm{A}-2$ Test (T-1323) is expressed in Eqn (2): $\mathrm{P} / \mathrm{S}_{\mathrm{y}}+\mathrm{Q} / \mathrm{S}_{\mathrm{y}}<=1$, with the restriction that the core temperature (average wall temperature) be less than the temperature at which $\mathrm{S}_{\mathrm{m}}=\mathrm{S}_{\mathrm{t}} @ 100 \mathrm{khrs}$.

Upon examination of Eqn (2), the A-2 Test is an average of the slow cycle solution and rapid cycle solution for the Bree problem, where the same cycle is repeated, e.g. $\mathrm{P}+$ $\left(\mathrm{Q}_{\mathrm{R}}\right)_{\max } \leq\left(\mathrm{S}_{\mathrm{y}}\right)$, where $\mathrm{S}_{\mathrm{y}}$ is the average of the yield strengths at the hot and cold ends of the cycle [6].

Weaknesses of the A-2 approach are as follows:

1. The A-2 Test is an average of rapid cycle and slow cycle solutions. As such, it relies upon favorable residual stresses (carry-over stresses that cancel out with subsequent applied stresses).

2. The A-2 Test is not clear as to whether the cycle definition is restricted to the 'whole life' or not.

3. No means are taken to ensure that the favorable residual stresses (carry-over stresses) form, and that they are indeed favorable as opposed to detrimental. 
4. No restriction is placed on the A-2 Test solution to ensure against the primary stress exceeding the hot yield strength, similar to the core stress not exceeding the hot yield strength in the B-1 Test.

5. While the use of an average of rapid cycle and slow cycle solution may tend to address some of the issues summarized above, assuming all residual stresses are beneficial, they are not considered to be sufficient to justify the use of the A-2 Test.

6. Use of other A-Tests in conjunction with the A-2 Test is unclear, and has no apparent justification.

The sole strength of the A-2 Test lies in the simplicity of the test; although, given the weaknesses indicated above, the simplicity is irrelevant.

\section{A-3 Test:}

The A-1 and A-2 Tests are tests intended to ensure loading in the elastic regime of the Bree interaction diagram. The A-3 Test (T-1324) is actually a shakedown approach.

Generally, shakedown approaches are less restrictive than elastic approaches; however, the A-3 Test also requires that creep strain accumulation be less than $0.2 \%$ and that creep damage (time fraction rule) is less than 0.1 with a core stress equal to the $1.5 \mathrm{~S}_{\mathrm{y}}(\mathrm{T}-1324)$. This is essentially the equivalent shakedown analysis of ASME Section III Subsection NB (NB-3222.2 and NB-322.3), where creep effects are not significant.

The maximum range of primary and secondary stress intensities $(\mathrm{P}+\mathrm{Q})$ must be less than an equivalent strength range, the lesser of $3 S_{\mathrm{m}}$ or $\overline{3 S_{m}}$. In cases where creep relaxation may occur, $\overline{3 S_{m}}$ is modified by use of relaxation strengths.

Weaknesses in the A-3 Test are:

1. This creep effective shakedown approach is more restrictive than both rapid cycle and slow cycle shakedown solutions, since the creep effective shakedown solution 
does not permit resetting of stresses at any time during the operation of the structure. Rapid and slow cycle shakedown solutions do not guarantee that stresses will not reset after creep relaxation. However, this is understood to be intentional.

Strengths of the A-3 Test are:

1. The approach is a simple screening test that may serve to justify no enhanced creep, no plastic ratcheting strains, and no significant creep effects to the extent that they reset stresses upon subsequent unloading and reloading.

\subsection{Simplified Inelastic Analysis}

\section{B-1 and B-3 Tests:}

The B-1 and B-3 Test are appropriate for problems with a constant primary membrane stress and cyclic through the wall secondary bending stress. They also are appropriate for conditions where yield strength varies with temperature. Due to the symmetry of the geometry and residual stress fields generated due to plastic deformation, there are very strong similarities between the rapid cycle and slow cycle Bree type interaction diagrams. Of particular importance is the recognition that since the B-1 and B-3 Tests are slow cycle solutions that predict an upper bound on the core stress for the Bree tube problem, the use of a slow cycle solutions requires additional restrictions.

These restrictions include restrictions on the core stress, where, if one wishes to avoid plastic ratcheting at the hot end of the cycle then the core stress can not exceed the hot yield strength. Also, for variable loading histograms, restrictions are placed on the value of the core stress from one cycle to the next; this is required to account for the lack of creep relaxation of the core stress that is in excess of the primary membrane stress during one cycle. If not accounted for in this manner, the residual stresses will carry over into subsequent cycles and can result in larger strain accumulation than predicted for subsequent cycles, specifically if the core stress in subsequent cycles is less than the core stress of a previous cycle. 
The S/P boundary in the B-1 and B-3 Test has a small error. The core stress is correct as indicated in the interaction diagram; but, there can be plasticity in the outer fibers below the indicated boundary ( $\mathrm{Y}=2)$, unless hot and cold yield strengths are equal. Otherwise, the proper $\mathrm{S} / \mathrm{P}$ boundary is at $\mathrm{Y}=1+\mathrm{S}_{\mathrm{yH}} / \mathrm{S}_{\mathrm{yL}}$.

The strength of the B-1 and B-3 Tests lies in the use of a slow cycle solution, which is also an upper bound on a slow cycle solution. The B-3 Test also provided a means of assessing severe cycles that generate plastic ratcheting strains. Furthermore, the restrictions placed on the use of the B-1 and B-3 Tests are appropriate for variable cycle loading. While not a weakness, the use of a less conservative but bounded slow cycle solution requires a little more effort to satisfy the additional restrictions. The weakness of the B-1 and B-3 Tests lie in its relevance to a specific type of problem, the Bree tube problem.

\section{B-2 Test:}

In contrast, the B-2 Test is a rapid cycle ratcheting solution based upon equal yield strength analysis. The geometry for which this simplified approach is based is the Bree tube; however, the use of a nonlinear thermal gradient is applied. The nonlinear thermal gradient was varied to represent various types of thermal shocks, e.g. a shallow but severe thermal shock, and a deep but mild thermal shock in another. The various thermal shocks were quantified in terms of their effective bending stress on the section.

Thousands of cases were simulated with equivalent bending stresses over the section, with each cases comprised of a range of thermal shocks as mentioned above. The maximum core stress obtained for the various thermal shocks was identified with the given effective bending stress. This was repeated with various levels of primary membrane stress, and the results plotted in terms of constant core stress contours, i.e. $\mathrm{z}$ contours. 
A strength with this approach is the simplified approach may very well be conservative; however, the weakness of the approach is that it has limited physical basis relative to actual geometries and loading conditions other than those for which the approach was developed. Furthermore, no guidance or interpretation is available to avoid plastic ratcheting at the hot end of a cycle in cases where the hot yield strength is significantly lower than that at the cold end of the cycle as in the B-1 and B-3 Tests.

\section{Strain Limits:}

All of the B-Tests rely upon use of the predicted core stress and isochronous curves in prediction of strain accumulation. The total strain accumulation at the core is restricted to $1 \%$. Limits are placed upon the bending ( $2 \%$ maximum strain at a surface) and at a local point (5\% maximum strain accumulation). Guidance is provided on how to obtain these strains, i.e. strain linearization. Two main issues exist with these requirements: the strain linearization procedure is not deemed appropriate, and even if appropriate, the linearization requires knowledge of the strain distribution across the section of interest, which is not available when one utilizes simplified analysis - rendering the simplified approaches of limited value. This topic will be covered to a greater extent in Subtask 9.5.

Integrating the ratcheting analysis and solution with the C-F assessment procedures would be ideal. If one utilizes one of the A-Tests, the elastic C-F procedures may be integrated with the ratcheting analysis. Otherwise, there is no such link or integration between the two procedures. While several other Gen IV Tasks are addressing C-F, these tasks are limited to assessment of procedures relative to material damage and life correlations. Such Tasks do not address procedural issues on how to perform structural analysis and obtain the inputs (stress and strain) to be utilized in the C-F life assessment. This is outside of the scope of this Task; however, the authors strongly recommend that this topic be addressed in the future. 


\subsubsection{R5 vs ASME}

R5 permits various analysis types, e.g. elastic analysis or inelastic analysis, to be utilized to satisfy both Primary Load Limits as well as Deformation Controlled Limits. This is in contrast to ASME NH only permitting elastic analysis for Primary Load Limits. ASME $\mathrm{NH}$ does recommend various simplified elastic analysis methods to satisfy Deformation Controlled Limits in Appendix T; ASME NH Appendix T also permits full inelastic analysis in replacement of such simplified methods. The difference between R5 and ASME NH with regards to use of inelastic analysis can be summarized as follows. The use of inelastic analysis to satisfy the intent of R5 design criterion is a bit more apparent, whereas it is not apparent in how to do so for ASME NH Appendix T. That said, opportunity exists to improve upon integration of inelastic analysis with the intent of design criteria for R5, ASME NH, and Appendix T.

\subsubsection{PRIMARY LOAD LIMITS}

\subsection{Elastic Analysis \& Limit Load Analysis}

Elastic analysis in R5 requires that the load history be resolved into different service cycles, with each service cycle having an associated cyclic load, a steady state load which operates during a dwell period, and a characteristic temperature. This is generally consistent with the intent of ASME NH, with the exception being that R5 provides some detailed advice on defining and constructing cycle types (Appendix A2 and A3). ASME provides very limited, if any, guidance on the subject. R5 also does not distinguish between different service load levels, while ASME NH does; this is important, since NH utilizes different safety factors within design criteria for different service levels. R5 is more of a Fitness-for-Service (FFS) Code, and does not distinguish between such levels.

In addition to elastic analysis, options exist that permit one to utilize reference stress analysis (limit load analysis) to satisfy Primary Stress Limits. Following the section of ASME, this section is organized according to a) the type of stress analysis, and b) the 
design criteria that incorporates the stress intensity and allowable stress to address specific failure mechanisms.

\section{Stress Analysis:}

Consistent with ASME NH, elastic analysis is conducted assuming a homogeneous body of parent material. The variation in stress with position $(\mathrm{x})$ and time $(\mathrm{t})$ is conducted for each cycle. Zones which give the most critical regions for lifetime limiting mechanisms (R5 specifically indicates: plastic collapse, creep rupture, ratcheting, creep-fatigue, and cyclically enhanced creep deformation) are selected. Special areas including weldments, maximum stress levels, stress ranges, and maximum temperatures at times at these temperatures are noted.

Maximum and equivalent stress and strain ranges are determined for each cycle in terms of von Mises equivalent stress and strain. Convenient sections through the thickness of the structure are selected, and the equivalent stress values Pm, PL, PB, Q, and F are determined. A definition for stress classification is provided, both in terms of a written description of their nature as well as guidance on how to calculate such values; several references are provided as well. While these are consistent with what the authors would assume ASME NH requires, $\mathrm{NH}$ does not provide as much detail or description on how to proceed with these steps, with the exception that ASME NH does provide tables that specify stress classification for various types of structures and loading. Given that there are a variety of methods by which to conduct stress linearization and classification, an opportunity for providing references that detail how to conduct such analysis exists; or, NH might provide specific recommendations on how to do so.

As with ASME NH, stress levels predicted with elastic analysis in R5 will result in more conservative designs (higher stresses) than if creep were taken into account. R5 does recognize this, similar to ASME NH, and takes this into account by permitting use of limit load or reference stress solution methods instead of elastic methods. These will be discussed in greater detail for specific failure mechanisms and design criteria. 


\section{Failure Mechanisms \& Design Criteria:}

For direct comparison with ASME NH, we revisit the following criteria in terms of the use of elastic stress analysis; reference stress methods are discussed separately. Of particular importance is the following fact: ASME NH utilizes the average temperature through the thickness when satisfying the following design criteria, while R5 utilizes the maximum temperature at the chosen section of interest.

- Limit load collapse under a single load application for time independent failure: primary membrane loading only

The primary membrane stress cannot exceed the time independent strength: R5 Eqn (6.1): $P_{m}<0.67 S_{y}{ }^{\prime}$; where $S_{y}{ }^{\prime}=S_{y}$ for creep stress exponents $n \geq 2$, and $\mathrm{S}_{\mathrm{y}}{ }^{\prime}=3 \mathrm{nS}_{\mathrm{y}} /\{2(\mathrm{n}+1)\}$ for $\mathrm{n}<2$. $\mathrm{S}_{\mathrm{y}}$ is the minimum monotonic $0.2 \%$ proof stress, at the maximum temperature at the chosen section. Typical values of $\mathrm{n}$ for ferritic and austenitic steels are in excess of 2; hence, typically $\mathrm{S}_{\mathrm{y}}{ }^{\circ}=\mathrm{S}_{\mathrm{y}}$. Note, $\mathrm{S}_{\mathrm{m}} \sim 0.67 \mathrm{~S}_{\mathrm{y}}$. Hence, this is consistent with the criteria provided by ASME NH with only exception being the definition of temperature; $\mathrm{R} 5$ is more conservative than ASME NH.

- Limit load collapse under a single load application for time independent failure: primary membrane and bending The primary membrane stress (including any local effects on membrane stress) cannot exceed the time independent strength: R5 Eqn (6.2): $\mathrm{P}_{\mathrm{L}}+\mathrm{P}_{\mathrm{b}}<\mathrm{S}_{\mathrm{y}}$. Again, for typical ferritic and austenitic materials, $\mathrm{S}_{\mathrm{y}}{ }^{\prime}=\mathrm{S}_{\mathrm{y}}$. Since $\mathrm{Sm} \sim 0.67 \mathrm{~S}_{\mathrm{y}}, \mathrm{R} 5$ effectively uses a $\mathrm{K}$ factor of 1.5 to account for stress redistribution due inelastic deformation. Thus, ASME NH and R5 are identical, with the exception of the use of maximum temperature by R5; R5 is more conservative than ASME NH.

- Deformation limit for time independent failure: primary membrane and/or primary bending

No specific criteria exists in R5; consistent with no specific criteria in ASME NH.

- Deformation limit for time dependent failure: primary membrane only

The primary membrane stress cannot exceed the time dependent strength: R5 has no criteria available that utilizes elastic stress analysis, only reference stress 
analysis. However, options are provided to allow one to use existing design codes, invert them, and arrive at estimations of reference stress approximations. In such cases, the reference stress is adjusted for local strain concentration to provide the rupture reference stress. Guidance is provided for isothermal, nonisothermal, and non-homogeneous structures, including addressing creep ductile vs. brittle materials.

It should be noted, R5 implements a 'rupture reference stress' (a stress allowable) that includes a limitation on strain accumulation (1\% creep strain (not total) for ferritics, and $2 \%$ creep strain for austenitics (not total strain)); this is similar to the use of $\mathrm{S}_{\mathrm{mt}}$ in ASME NH, with the noted exception that ASME NH includes a limitation on the onset of tertiary creep.

- Excessive creep deformation and rupture limit for time dependent failure: primary membrane only

The primary membrane stress cannot exceed the time dependent strength: again, R5 has no equivalent elastic stress analysis method and design criteria to address this topic. The process of inverting design criteria in available Code to arrive at an equivalent reference stress is permitted.

- Excessive creep deformation and rupture limit for time dependent failure: primary membrane and bending

The primary membrane and bending stress cannot exceed the time dependent strength: again, R5 has no equivalent elastic stress analysis method and design criteria to address this topic. The inversion process stated in the previous paragraph may be applied to address this topic. The inversion of ASME NH has been conducted already by McGreevy [3]; rather than defer the summary of this work until Task 9.4 (as the inversion has not been incorporated into any Code), it does make sense to include it herein, rather than later. McGreevy's equivalent ASME NH recommendation for time dependent primary load limits is $\sigma_{r e f} \leq S_{m t}$; if one includes primary bending as well, the ASME $\mathrm{NH}$ recommended equivalent, 
taking into account stress redistribution at fibers, is: $\sigma_{r e f}\left(\frac{2 n+1}{2 n}\right) \leq S_{t}$, where $\mathrm{n}$ is the creep exponent of the material.

Note, similar to ASME NH, R5 addresses cases where the cycle time is less than the total service life by the use of a creep usage factor, $U$. The creep usage factors are similar between ASME NH and R5, with the only exception being that R5 only considers the portion of the steady loading operation where creep is significant (ASME NH requires the entire duration of the cycle to be considered?), and R5 utilizes the maximum temperature of the section in question while ASME NH considers the average temperature in the section. Of course, differences in database or approach for determining allowable stresses (e.g. AMSE NH using a restriction based upon onset of tertiary creep) may exist as well.

\subsection{Reference Stress Methods}

R5 to a large extent is based upon implementation of the reference stress approach. In contrast, ASME uses no such concepts. The various failure criteria reviewed earlier are revisited relative to the use of the reference stress.

- $\quad$ Limit load collapse under a single load application for time independent failure: primary membrane loading only

The primary membrane stress cannot exceed the time independent strength: R5 has an option (Option 3), which permits a lower bound limit analysis (or use of an existing solution) to demonstrate that the limit load is always greater than the applied mechanical load for a material yield strength of $0.67 \mathrm{~S}_{\mathrm{y}}$ '. Given that $\mathrm{S}_{\mathrm{m}} \sim 0.67 \mathrm{~S}_{\mathrm{y}}$, this equates to $\mathrm{P}<0.67 \mathrm{P}_{\mathrm{y}}$, where $\mathrm{P}$ is the applied mechanical load for the cycle of interest, and $\mathrm{P}_{\mathrm{y}}$ is the limit load of the structure. For isothermal structures under application of primary loads $\mathrm{P}, \sigma_{\text {ref }}=\mathrm{PS}_{\mathrm{y}} / \mathrm{P}_{\mathrm{u}}$. This is equivalent to $\sigma_{r e f} \leq S_{m}$. 
- Limit load collapse under a single load application for time independent failure: primary membrane and bending

The primary membrane stress (including any local effects on membrane stress) cannot exceed the time independent strength: R5's Option 3 applies directly to this criterion as well.

- Deformation limit for time independent failure: primary membrane and/or primary bending

No specific criteria exists in R5; consistent with no specific criteria in ASME NH.

- Deformation limit for time dependent failure: primary membrane only

The primary membrane stress cannot exceed the time dependent strength: R5 does not provide specific criteria that address deformation limits. Since the creep rupture stress includes a limitation on permissible creep strain in addition to failure by rupture, the rupture limit for time dependent failure would address this criterion. This is true for ASME NH as well.

- Excessive creep deformation and rupture limit for time dependent failure: primary membrane only

The primary membrane stress cannot exceed the time dependent strength: R5 does not separate primary membrane stress design criteria separately from primary membrane and bending; similar to the time independent criteria discussed above. The reference stress considers the impact of all primary loads simultaneously. For example, for isothermal structures of rectangular crosssection, an overestimate of the reference stress is given in R5 Eqn (A5.2): $\sigma_{\text {ref }}=\left[\left(\mathrm{P}_{\mathrm{B}} / 3\right)+\left\{\left(\mathrm{P}_{\mathrm{B}} / 3\right)^{2}+\mathrm{P}_{\mathrm{L}}^{2}\right\}^{1 / 2}\right]$. It is noted, that this equation neglects redistribution of stresses from one section to another due to inelastic deformation. R5 permits a significant number of approaches to be used to obtain reference stress solutions: approaches are discussed in R5 (Volume 4/5 Appendix A2, Volume 6 Appendix A2, Volume 7 Appendix A3), R6 (Sections 3.8, II.4, and IV.1), compendia of limit load solutions such found in R6 (II.4), and other similar sources, inversions of design Code, limit analysis, solutions from the literature, finite element analysis, and experiments. 
For non-isothermal structures, the reference stress is obtained as follows, per section A5.1.2 of R5. Two options exist.

(1) Pessimistically speaking, the reference temperature can be set equal to the highest temperature in the structure; then, apply the isothermal reference stress procedures.

(2) When the temperature $T$ varies with position $x$, values are assigned to the yield strength that are in proportion to the minimum creep rupture stress $S_{R}$ for the part $t_{i}$ of the operational lifetime which is at temperature $T$ in the creep range: $\sigma_{\mathrm{y}}(\mathrm{x}) \sim \mathrm{S}_{\mathrm{R}}\left\{\mathrm{T}(\mathrm{x}), \mathrm{t}_{\mathrm{i}}\right\}$. This is equivalent to conducting a limit load calculation on a structure who's effective yield strength is both time and temperature dependent, e.g. $\sigma_{\mathrm{y}}(\mathrm{x})=\min \left[\operatorname{Sy}\{\mathrm{T}(\mathrm{x})\}, \mathrm{S}_{\mathrm{R}}\left\{\mathrm{T}(\mathrm{x}), \mathrm{t}_{\mathrm{i}}\right\}\right]$. This results in a single value of limit load, $\mathrm{Pu}$; however, the reference stress varies spatially, $\sigma_{\text {ref }}(\mathrm{x})$, with the associated reference temperature $\mathrm{T}_{\text {ref }}$ and position $\mathrm{x}$. Since the effective yield strength is determined based upon the same time $t_{i}$, the reference stress and temperature at any location in the structure may be selected, as all such possibilities result in the same creep rupture endurance. Thus, any selection is representative of the structure or feature as whole; R5 recommends that the maximum stress or maximum temperature location be utilized. The same approach applies for structures made from different materials.

- Excessive creep deformation and rupture limit for time dependent failure: primary membrane and bending

The primary membrane and bending stress cannot exceed the time dependent strength: again, R5 does not separate primary membrane stress design criteria separately from primary membrane and bending. The reference stress approach described above for time dependent failure: primary membrane only are used. 
Note, similar to ASME NH, R5 addresses cases where the cycle time is less than the total service life by the use of a creep usage factor, $U$. The creep usage factors are similar between ASME NH and R5, with the only exception being that R5 only considers the portion of the steady loading operation where creep is significant (ASME NH requires the entire duration of the cycle to be considered?), and R5 utilizes the maximum temperature of the section in question while ASME NH considers the average temperature in the section. Of course, differences in database or approach for determining allowable stresses (e.g. AMSE NH using a restriction based upon onset of tertiary creep) may exist as well.

- Non-ductile fracture:

R5 permits use of materials that are creep brittle (A1.7). R5 provides a list of steels that are considered creep ductile (A1.7), which exclude weld metals and heat affected zones. These include a variety of steels in normalized and tempered or annealed conditions. Among them are:

o Carbon-manganese steels

o $1 / 2 \mathrm{CrMoV}$

o $1 \mathrm{Cr}^{1} \frac{1}{2 \mathrm{Mo}}$

o $2 \frac{1}{4} \mathrm{Cr} 1 \mathrm{Mo}$

o 9Cr1Mo

o $12 \mathrm{Cr} 1 \mathrm{MoV}(\mathrm{W})$,

Solution treated austenitic stainless steels of the 300 series include:

o Types 304, 304H, and 304L

o Types 316, 316H, 316L, and 316L(N)

o Types 321 and $312 \mathrm{H}$

o Alloy 800 and $800 \mathrm{H}$ 
Note, no time, temperature, or stress limits are discussed relative to these materials. Such restrictions are assumed to be included in the stress allowables published by British Energy, e.g. the AGR materials data handbook, R66. R5 permits other justified sources of materials data to be used where appropriate.

If materials are not listed, the designer must consider a criterion to determine if the material is creep ductile or creep brittle. This criterion essentially states that the average creep rupture strain at failure must be equal to or greater than five (5) times that of the average Monkman-Grant strain, at the given temperature and stress level in question.

R5 and R6 provide extensive guidance on assessment of structures with defects, both at elevated temperature, and at temperatures where creep is insignificant. This topic is outside of the scope of this Task; although, a closely related Task is underway within the Gen IV Materials Tasks.

\section{- Failures of Weldments:}

In ASME NH, the time dependent effects of weldments are addressed by modification of allowable stresses (NH-3221) by definition of St, where St $=$ min (St in Tables I-14.4, $0.8 * \mathrm{~S}_{\mathrm{r}}{ }^{*} \mathrm{R}$ ), where $\mathrm{R}$ is the ratio of weld metal creep rupture strength to base metal creep rupture strength (note, this factor apparently only addresses rupture, not time to $1 \%$ strain or onset of tertiary creep).

In R5, the treatment of weldments in terms of creep deformation and damage is still at a formative stage. The procedures and principles are very similar to that for parent materials, but some guidance related to the following complications are provided:

o potential mismatch of material properties,

o welding defects,

o high local residual stresses,

o effects of surface finish creating ‘dressed' vs. 'undressed’ welds. 
Appendix A4 of R5 addresses such issues. Specific to Primary Stress Limits, the same procedure utilized for parent material applies; the exception being that the rupture reference stress for a feature consisting of more than one material is utilized. If stress redistribution between regions of the weldement with different creep strengths exist, Volume 4/5 and Volume 7 contain recommended procedures for appropriate determination of weldment limit loads. These approaches are largely based upon reference stress approaches, not elastic stress analysis. While outside of the scope of this Task, Appendix 4 also contains guidance on cyclic assessment of weldments, including the use of fatigue strength reduction factors (FSRF).

- Multiaxial effects on Creep Failure:

R5 includes a means to account for multiaxial stress rupture. The approach was proposed by Huddleston [7]. Caution is indicated, since some materials may fail due to maximum principal stresses than equivalent stresses - requiring an alternative multiaxial rupture criterion (not provided by R5).

\subsubsection{DEFORMATION CONTROLLED LIMITS}

R5 does not specifically refer to deformation limits by name; rather, they are referred to as ‘shakedown analysis and secondary stress limits'. This includes limitation on ratcheting, cyclically enhanced ratcheting, and creep-fatigue. The use of the term 'elastic' must be used carefully when discussing elastic methods vs. inelastic methods in R5; this statement will be elaborated on below.

R5 presents and implements design criteria based upon well established and understood shakedown theory. The presentation of this theory is in the following context: stresses are represented in terms of the elastically calculated stresses, and residual stresses. Typically, R5 disregards peak stresses (F) in the following implementation; if peak stresses are included, results will be more conservative. 
If the structure is shown to remain elastic, the residual stresses are zero. To satisfy shakedown, inelastic deformation sets up residual stress fields in the structure. If these residual stress fields remain constant upon subsequent repetition of loading of the structure, a steady cyclic shakedown state is reached. If these residual stresses do not remain constant, then the structure either experiences plastic loading (reversed plasticity) or plastic ratcheting.

With this in mind, it is difficult to compare simplified methods in R5 relative to ASME $\mathrm{NH}$ in terms of 'elastic analyses' or 'simplified elastic methods'. However, comparisons are more readily made in the context of the cyclic steady state of the structure, e.g. elastic, shakedown, plastic, or ratcheting.

One very significant point to be made is with regards to the shakedown reference stress and the elastic core stress. One must be careful interpreting the use of the shakedown reference stress that R5 utilizes relative to the use of the term core stress as developed and implement in ASME NH Appendix T. The two are not equivalent. There are some similarities, and under certain circumstances the two have the same value. However, the reader should be aware that they are not consistently equivalent in meaning at any point in time. This will be discussed at length later in this report. The ratcheting reference stress is actually consistent with the meaning of the core stress. R5 does not currently utilize a 'ratcheting reference stress'; this topic will be reviewed in Subtask 9.4.

Treatment of Residual Stresses, Shakedown Reference Stress vs Core Stress, and Rapid vs Slow Cycle Solutions:

Of particular importance is the realization of the source of the residual stresses that lead to shakedown, plasticity, or ratcheting. In R5, the residual stresses are due to redistribution of stresses due to plastic deformation; creep is not permitted to take place. Hence, the cyclic analysis in R5 is considered as 'rapid cycle analyses'. If shakedown is demonstrated (or elastic loading), the resulting reference stress is deemed the 'shakedown 
reference stress'. R5 does not permit loading into the plasticity or ratcheting regimes; if the structure fails to shakedown, inelastic analysis must be used, or the load cycle is deemed unacceptable.

There is one exception to the source of residual stresses in R5: the beneficial aspect of creep relaxation on subsequent stresses at the hot end of the cycle. Creep relaxation tends to reduce the stress at the hot end of the cycle, i.e. generating additional residual stresses due to creep relaxation. R5 takes a simplified approach in an attempt to account for reduction of these stresses at the hot end of the cycle due to creep deformation, with the intention of reducing the calculated creep damage as well as the enhanced strain range due to creep; the overall intent is to improve upon the C-F life prediction. While C-F is out of scope in this report, the approach is discussed herein relative to adverse implications on the prediction of the core stress.

In brief, to address creep effects on residual stresses R5 utilizes time and temperature dependent effective yield strengths to conduct rapid cycle shakedown analyses. If one can demonstrate that the structure achieves shakedown, then one is guaranteed that even with full relaxation of secondary stresses after startup, stresses will not reset upon subsequent shutdown and startup. If one includes peak stresses, $F$, in the shakedown analysis, then the structure effectively is not aware that it is undergoing cyclic loading. For very high temperature applications, this simplified approach can and will be grossly conservative. The maximum stress predicted in the structure (e.g. at the extreme fiber for the Bree tube problem) will decrease with decreasing yield strength; however, this also increases the predicted core stress. This topic will be discussed further, but later on in this section.

ASME NH Appendix T, on the other hand, includes approaches that are based upon residual stresses due to plastic redistribution alone (A-Tests and the B-2 Test), while some approaches are based upon residual stresses that arise from both plastic and creep redistribution of stresses (B-1 \& B-3 Test). Note, the B-1 and B-3 Tests are not rapid cycle solutions, but rather slow cycle solutions. All of the A-Test and B-Test approaches 
are intended to address the behavior of the elastic core region, specifically enhanced cyclic creep deformation (ratcheting) of the core.

Extension of the simplified methods (A-Tests or B-1 Tests) for enhanced cyclic creep ratcheting to elastic C-F life prediction methods is one approach in Appendix T. The core stress obtained from the A-Tests or B-Tests is then used to predict the creep strain increment (creep strain range contribution to the total strain range of the cycle). The creep strain range increment is multiplied by the local geometric stress concentration factor, $\mathrm{K}^{1}$.

This is quite a different approach as compared to R5's use of the shakedown reference stress, which essentially is the maximum stress in the structure at the start of dwell, e.g. the fiber stress in the Bree tube problem. The shakedown reference stress appears to be intentionally utilized with the intent in assessing creep-fatigue damage in subsequent calculations, with no apparent intent to consider the core stress and its impact on the overall deformation or strain in the structure.

However, R5 also refers to the use of a Bree type of solution (when the geometry and loading case are consistent with the Bree solution) to obtain the core stress; and in this specific case, the core stress is specifically referred to and noted to be a less conservative estimate of the shakedown reference stress ${ }^{2}$. There appears to be inconsistency on the use and intent of the shakedown reference stress in R5. In short, one can not and should

\footnotetext{
${ }^{1}$ Note: a problem is easily observed with the elastic C-F approach in ASME Appendix T. Consider T-1432 (g): while load controlled stresses may be zero for a simple thermal bending stress alone, also resulting in a core stress of zero, the resulting creep strain increment $\Delta \varepsilon_{\mathrm{c}}$ predicted with the recommended procedure will be zero. This is clearly incorrect; while the core strain may be zero, the creep strain increment at the surface (with or without a local stress concentration) will be greater than zero. Unless there is a constraint, e.g. no warping of sections so that total strain remains constant, then there can be creep during relaxation of secondary stresses that enhance the strain range during the cycle. Furthermore, in cases where the core stress is nonzero, T-1432 (g) utilizes a load controlled solution, meaning an infinite elastic follow-up factor. This will be very conservative for most cases. Note, creep damage follows a different procedure in ASME NH Appendix T, where T-1433 permits the stresses to relax when assessing creep damage, e.g. T1433 (a) Step 5, or T-1433 (b). Ideally, creep damage and the contribution of the creep strain increment to the strain range should be handled consistently; furthermore, assumption of infinite elastic follow-up is extremely conservative for many circumstances, as is the assumption of uniaxial relaxation inappropriate in many circumstances.

${ }^{2}$ To clarify, the shakedown reference temperature, $\mathrm{T}_{\text {ref }} \mathrm{s}$, is equal to the maximum wall temperature, not the average wall temperature as in ASME Appendix T. Furthermore, R5 utilizes a temperature dependent yield strength rapid cycle solution for this core stress; it has been clearly demonstrated that this approach is grossly conservative for very high temperature applications - specifically when creep relaxation is significant [McGreevy?].
} 
not utilize a shakedown reference stress to consistently represent the core stress and the stresses at the surface of the section of interest, e.g. at the inner or outer fiber. Doing so will lead to conservative designs.

Closer examination of the application of the shakedown reference stresses and core stresses reveals that only when the loading causes the structure to approach the shakedown-ratcheting boundary do the shakedown reference stress and the core stress levels become equal in value; otherwise, the shakedown reference stress will always be greater than the core stress. For example, in the Bree tube problem, the stresses at either the inner or outer fibers will reach yield prior to that of the core. As one approaches the ratchet boundary, the core stress increases until it reaches the yield strength and ratcheting commences. Furthermore, since the shakedown reference stress is not indicative of the core stress, the reference temperature associated with the shakedown reference stress will be more closely associated with the maximum metal temperature, rather than the mid-wall temperature that is associated with the core stress.

If one wishes to address and examine the behavior of the core region, then the ratchet boundary, and hence ratcheting reference stress, is an appropriate reference stress. If one wishes to address the creep-fatigue behavior of the structure, e.g. the extreme fibers in the Bree tube problem, then a shakedown reference stress is more appropriate.

Unfortunately, the Bree diagram that is in R5 is not strictly correct for a rapid cycle solution with temperature dependent yield strength. The correct solution was recently provided by McGreevy [4]. The difference or error observed is that the shakedownplasticity boundary indicated by $\mathrm{R} 5$ is conservatively indicated at $\mathrm{Y}=2 \rightarrow$ at $\mathrm{Q}=2 * \mathrm{~S}_{\mathrm{yH}}$; strictly speaking, when normalizing $\mathrm{Y}_{\text {by }} \mathrm{S}_{\mathrm{yH}}$ (as in R5) the boundary should be at $\mathrm{Y}=1+\mathrm{S}_{\mathrm{yL}} / \mathrm{S}_{\mathrm{yH}} \rightarrow$ at $\mathrm{Q}=\mathrm{S}_{\mathrm{yH}}+\mathrm{S}_{\mathrm{yL}}$. Hence, the permissible shakedown region in $\mathrm{R} 5$ is unnecessarily restricted further. 
Returning to the use of an effective yield strength that is both time $\left(\mathrm{t}_{\mathrm{s}}\right)^{3}$ and temperature dependent to arrive at a shakedown reference stress, the permissible operating conditions may be overly restricted as well. In other words, one might not be able to demonstrate for a given load cycle that the structure achieves shakedown with an effective yield strength that is both time and temperature dependent $\underline{\text { and }}$ the assumption of a rapid cycle. Failure to demonstrate that the structure achieves shakedown indicates that after the time $t_{s}$, the subsequent reversal of cyclic loads will cause stresses to reset.

If the shakedown-plasticity boundary is crossed, this means that there will be a plastic strain range associated with the total strain range of the cycle. If the shakedown-ratchet boundary is crossed, this means that that the core stress exceeds the temperature and time dependent strength criterion, e.g. the core stress exceeds $S_{t}(A S M E N H)$ or $S_{R}(R 5)$.

Note, this core stress is achieved by use of a rapid cycle analysis; it is a conservative estimate of the core stress. A less conservative but bounded estimate of the core stress is available. With significant creep relaxation, much more beneficial residual stresses are achieved that setup a significantly different cyclic steady state than predicted by R5's rapid cycle methods. As a result, a larger operating region is possible for which the structure still demonstrates shakedown.

Note, this definition of shakedown is not identical to that of 'short-term shakedown' with or without time dependent yield strength. This definition of shakedown is one where the structure after full relaxation of residual stresses at the hot end of the cycle, is subjected to further cyclic loading. The additional cyclic loading is conducted without any creep. During the subsequent loading, the structure may exhibit 'short-term shakedown'. In other words, in the Bree tube problem, the slow cycle solution for shakedown reveals operating conditions where after the full relaxation of stresses at the hot end of the cycle, the structure will shakedown by one of two general cases. Either the structure will yield

\footnotetext{
${ }^{3}$ This is not R5 nomenclature, but for clarity in further discussion we define this term.
} 
only the $1^{\text {st }}$ subsequent shutdown $\left(\mathrm{S}_{1}\right)$ or it will yield only on the $1^{\text {st }}$ subsequent shutdown and startup $\left(\mathrm{S}_{2}\right)$. The structure will behave elastically under all subsequent cycles ${ }^{4}$.

This significantly different cyclic state is the 'slow cycle' solution $[8,5,9]$. The solution provides a bound on the core stress for the slow cycle. For structures that undergo uniform primary membrane loading and cyclic through the wall linear thermal bending stresses, the B-1 Test actually is the 'slow cycle' solution.

Figure 9.3.2.1 illustrates the difference between rapid and slow cycle solutions for shakedown and ratcheting boundaries, when the yield strength is a function of temperature only (for the case where $\mathrm{S}_{\mathrm{yL}}=2 * \mathrm{~S}_{\mathrm{yH}}$ ). Note, the B-1 Test indicates that the slow cycle solution shakedown boundary is at $\mathrm{Y}=2$; this is unfortunately incorrect. The correct boundary is at $Y=1+1 / \alpha=1+S_{y H} / S_{y L}\left(\alpha=S_{y L} / S_{y H}\right)$, since in this case $S_{y L}$ is used to normalize the axes. While not readily apparent, Appendix $\mathrm{T}$ does properly state that the slow cycle ratchet boundary for this case is when the core stress equals the hot yield strength ( $\left.\mathrm{S}_{\mathrm{yH}}\right)$, i.e. for $\alpha=2$ this is for stress contours where $\mathrm{z}=0.5$. When the temperature dependent yield strengths are equal, the rapid and slow cycle solutions are identical as illustrated by the blue dashed lines in Figure 9.3.2.2.

Now, consider the case where the time independent yield strengths differ by a factor of 2 , i.e. $\alpha=2$. Figure 9.3.2.2 illustrates the shakedown and ratchet boundaries for the Bree tube problem. The thin red lines are the slow cycle solution boundaries, while the thick red lines are the rapid cycle solution boundaries. The operating point in question is given by the large solid black circle, and is clearly within the shakedown boundary of the rapid and slow cycle temperature dependent yield strength boundaries.

If one utilizes a temperature and time dependent yield strength, which effectively reduces the hot yield strength such that $\alpha=3.9$, one arrives at the thin and thick green lines for rapid and slow cycle solutions, respectively. This is the approach that R5 permits to

\footnotetext{
${ }^{4}$ All subsequent cycles refers to the repletion of the same load cycle setting up a steady cyclic state.
} 
reduce the shakedown reference stress. The values of the stress contours, $\mathrm{z}$, are defined as $\mathrm{z}=\sigma_{\mathrm{c}} / \mathrm{S}_{\mathrm{yL}}$, where $\sigma_{\mathrm{c}}$ is the core stress. Note, $\mathrm{S}_{\mathrm{yL}}$ does not change.

The interpretation of the thick green line (R5's rapid cycle time and temperature dependent yield strength) is that the shakedown reference stress will be equal to $0.25 * S_{\mathrm{yL}}$. Since the operating point intersects the shakedown-ratchet boundary, the predicted core stress becomes equal to the predicted shakedown reference stress. In contrast, the rapid cycle time independent solution, Figure 9.3.2.3, illustrates the same operating point, which falls on the stress contour of $\mathrm{z}=0.28$, or $\sigma_{\mathrm{c}}=0.28 \mathrm{~S}_{\mathrm{yH}}=0.28 \mathrm{~S}_{\mathrm{yL}} / 2=0.14 \mathrm{~S}_{\mathrm{yL}}$. This illustrates that the time dependent yield strength increases the core stress substantially.

In reality, the core stress in the steady cyclic state can be lower than that predicted by the rapid cycle time independent analysis. The slow cycle solution provides a bound on the steady cyclic state. Figure 9.3.2.4 illustrates the stress contour of $\mathrm{z}=0.10$ for the slow cycle solution; the operating point lies slightly to the left, at about $\mathrm{z}=0.09$. Hence, the steady cyclic state core stress is equal to $0.09 \mathrm{~S}_{\mathrm{yL}}$. This is about three (3) times lower than predicted by the rapid cycle time and temperature dependent yield strength solution, and about one and a half (1.5) times lower than the rapid cycle time independent yield strength solution.

The implications are that if one desires to assess the accumulation of strain (or possible rupture) of the core region, the use of time and temperature dependent yield strength rapid cycle analysis is overly conservative. If one desires to assess whether or not a structure, after creep relaxation for a period $t_{s}$, upon subsequent shutdown and startup will yield and reset stresses or not - then the time and temperature dependent yield strength can conservatively answer this question; if peak stresses are not included in the shakedown assessment, then stresses may reset, but the extent will be smaller and subsequently the strain range predicted in the area of interest will be smaller. However, this assessment of resetting of stresses after creep relaxation is also prone to overconservatism. 
Closer examination of Figure 9.3.2.3 or Figure 9.3.2.4 reveals that the slow cycle solution predicts the operating point of interest to lie within the elastic regime. This means that after initial loading, which may or may not include a small amount of plastic deformation, and subsequent creep relaxation, the structure will always behave in an elastic manner. The rapid cycle time and temperature dependent analyses predicts that the maximum duration of the hold time where creep stresses relax is $t_{s}$ such that $\mathrm{S}_{\mathrm{y}}(\mathrm{T}, \mathrm{ts})=\mathrm{S}_{\mathrm{yL}} / 3.9$; the slow cycle solution reveals that there is no limitation on the time duration to ensure that stresses do not reset. Note, this does not mean that the structure has an infinite life; in fact, life is limited by the allowable stress $\left(S_{t}\right.$ in Appendix $T$, or $S_{R}$ in R5).

Finally, slow cycle solutions will also reduce the stress at the start of dwell, providing a more reasonable estimate on the creep strain increment during the cycle, and the subsequent total strain range and creep damage calculations for creep fatigue assessment.

This leads into the topic of the use of elastic follow-up factors to address the nature of load controlled vs. displacement controlled creep during the dwell periods of the cycle. Use of this elastic follow-up factor provides conservative but reasonable estimates of the creep strain increment during the dwell period, as opposed to a variety of methods that may have overly conservative estimates, e.g. ASME NH Appendix T elastic C-F approach in T-1432 which assumes load controlled stresses to assess the creep strain increment. 


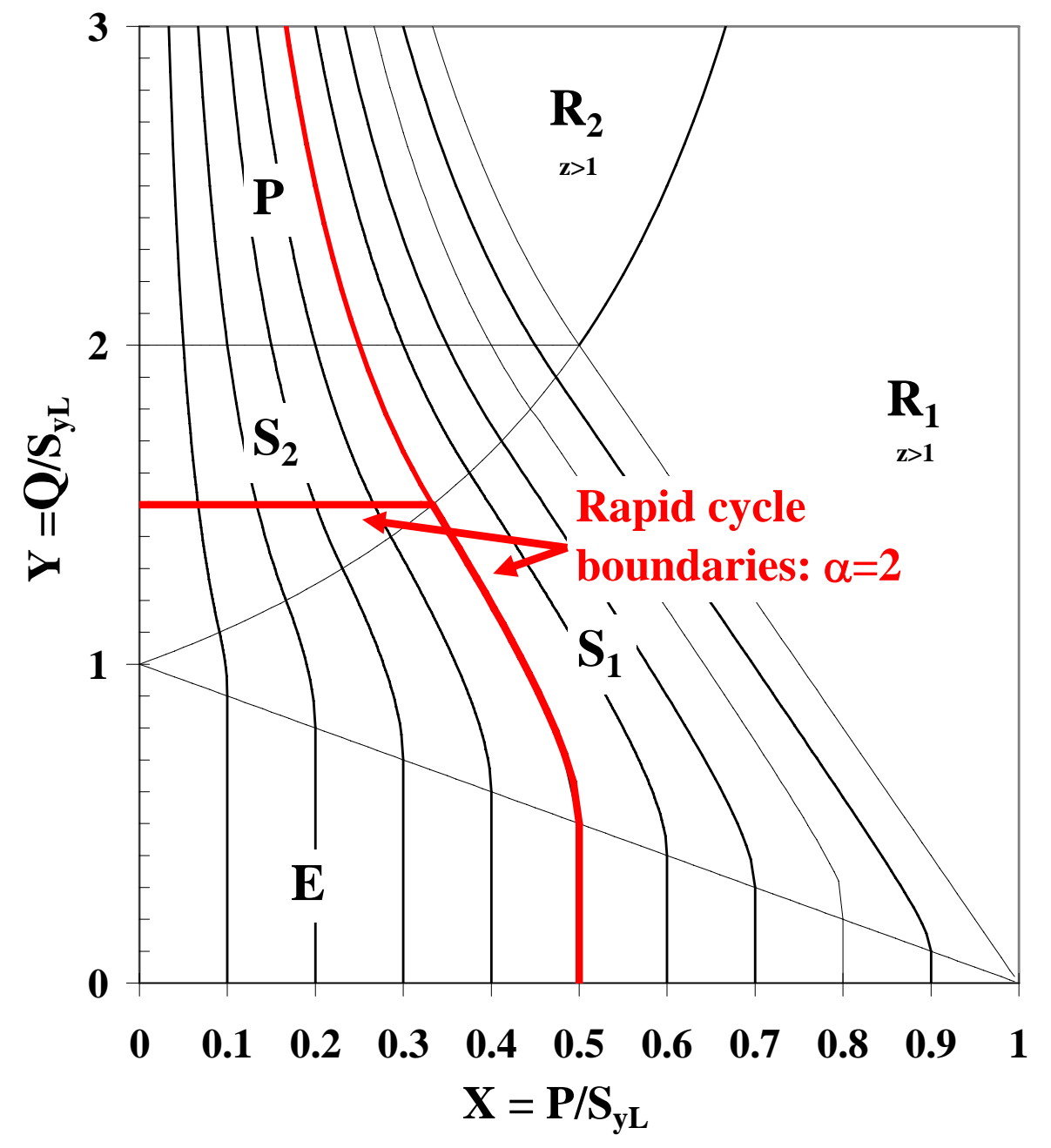

Figure 9.3.2.1 Boundaries for Bree tube problem: rapid cycle \& slow cycle solutions, $S y=f(T)$ only; $S_{y L}=2 * S_{y H}$ 


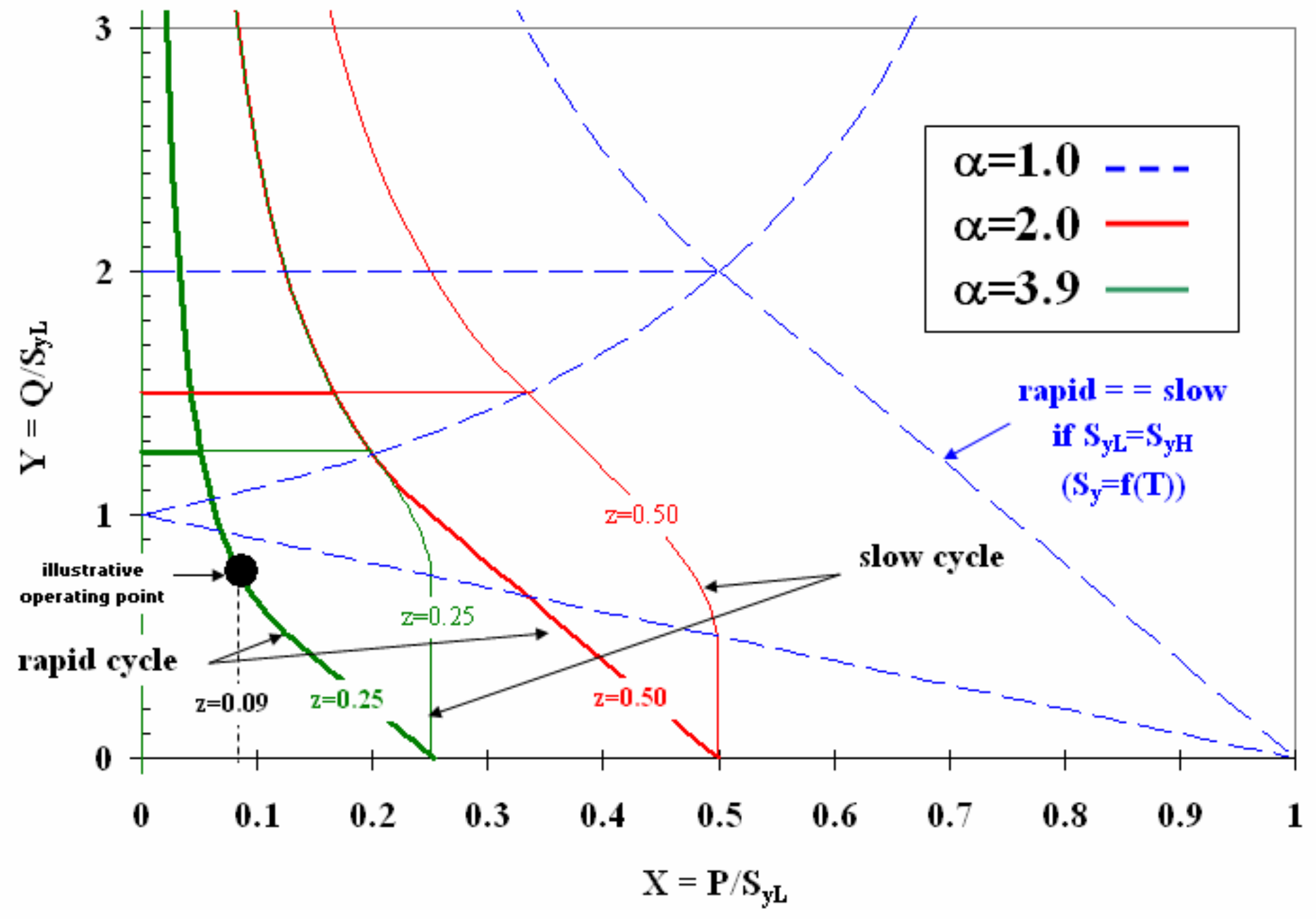

Figure 9.3.2.2 Impact of temperature dependent and time dependent yield strength on ratchet boundaries 


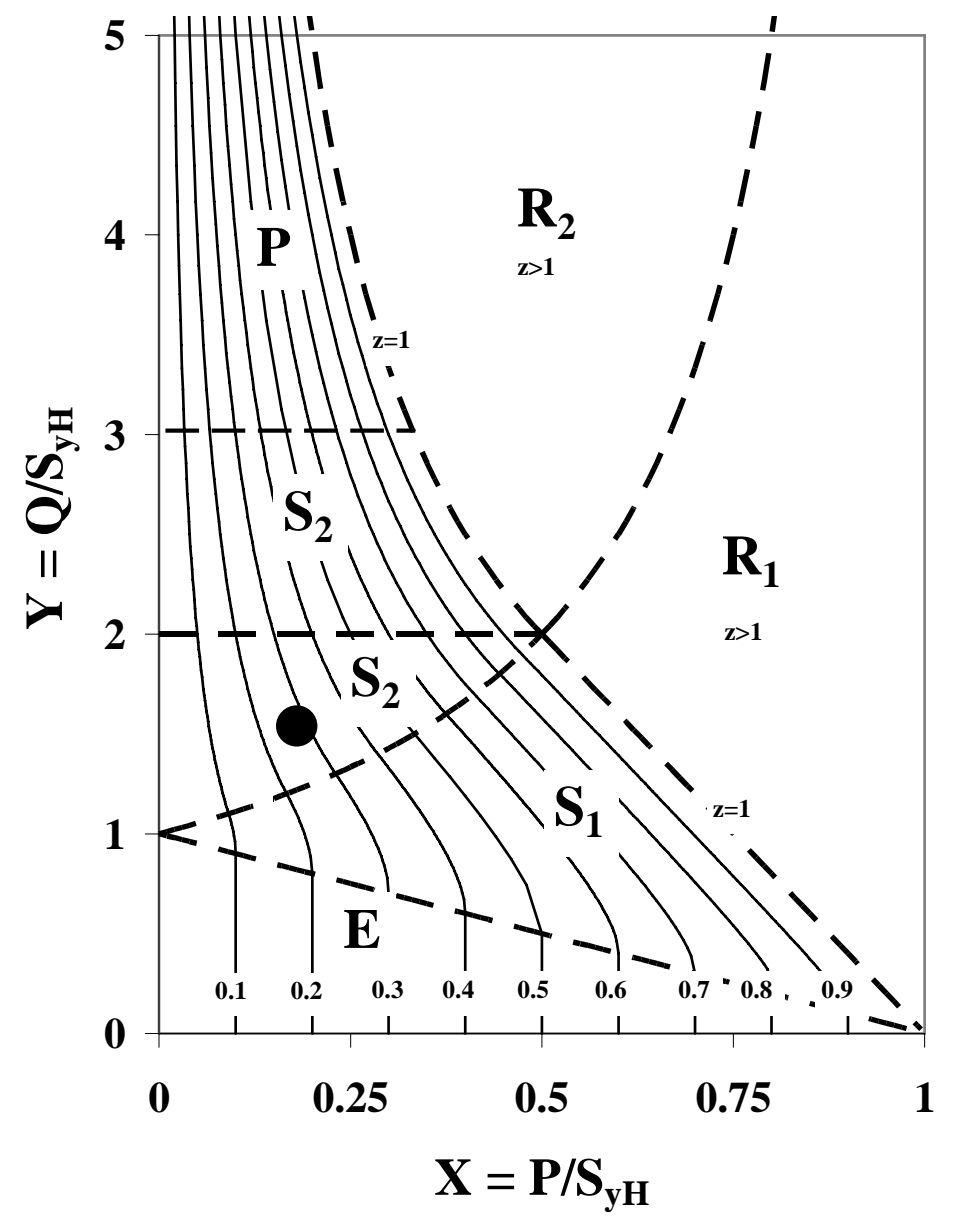

Figure 9.3.2.3 Operating point and contours of constant core stress for rapid cycle temperature dependent yield strength Bree tube problem 


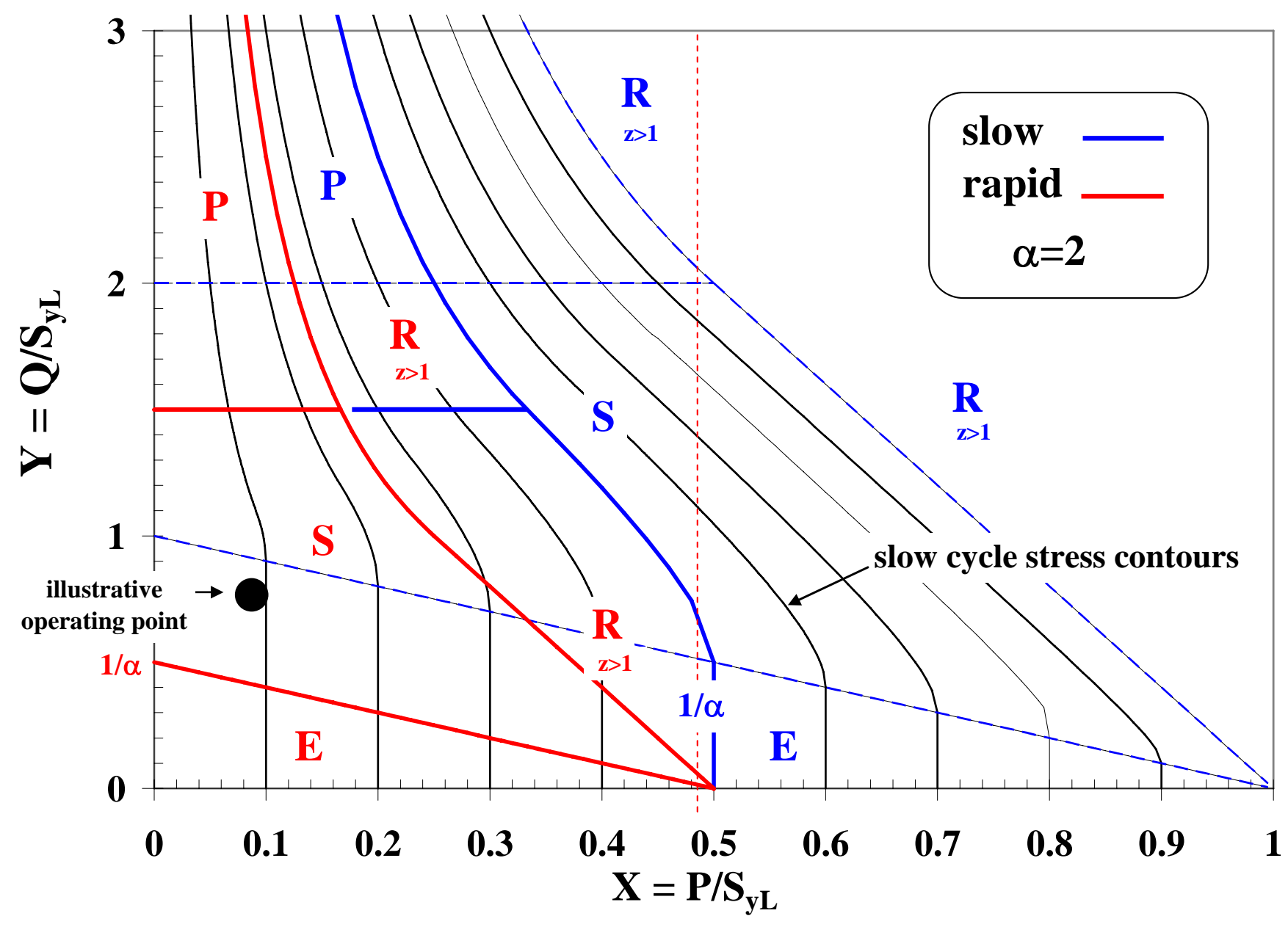

Figure 9.3.2.4 Operating point and constant core stress contours for slow cycle bounded solution with temperature dependent yield strength elastic, shakedown, and ratchet boundaries 
Another key aspect is consideration of the effects of cycle sequence. In R5, these sequence effects are initially ignored, e.g. load cycles are treated independently. Appendix A12 provides guidance on inelastic analysis methods to consider cycle order

effects on deformation; Appendix A10 describes approaches for taking into account cycle sequencing on fatigue damage accumulation.

\subsection{Elastic Behavior (Rapid Cycle)}

The elastic behavior case is a subset of the shakedown reference stress solution, i.e. this is the special case when the residual stress is constant and is zero. If one can show that the equivalent elastic stresses determined from linearized stresses, at all the points $\mathrm{x}$ on the stress classification line for all time t, are within a modified yield limit:

$$
\bar{\sigma}_{e l, l i n}(x, t) \leq \mathrm{K}_{s} S_{y} \quad \text { (R5: Eqn 6.11) }
$$

the structure satisfies 'global shakedown'. $\mathrm{K}_{s} S_{y}$ is a measure of the ability of the material to develop a steady cyclic behavior, $S_{y}$ is the minimum $0.2 \%$ proof stress for the material for the temperature at point $\mathrm{x}$ and time $\mathrm{t}$.

This case is very similar to ASME NH Appendix T, A-1 and A-2 Tests, with only a slight difference in the yield strength that dictates the limit of elastic loading. Note, this is a rapid cycle analysis.

One significant difference is that R5 also permits a looser definition for global shakedown - if the cyclic plastic zone is less than $20 \%$ of the section thickness and the elastic region satisfies Eqn 6.11, then shakedown is met. In such a case, it is necessary to assume that the steady cyclic stresses are equal to the elastic stresses. Appendix T has no such consideration or rule.

Similar to the A-3 Test, R5 has a check for insignificant cyclic loading. This check must be met to essentially receive an exemption from C-F analysis. To do so, one must satisfy global shakedown, demonstrate that fatigue is insignificant, and demonstrate that creep 
behavior is unperturbed by cyclic loading. (One may also demonstrate insignificant cyclic loading if they chose to conduct detailed shakedown analysis requiring assessment of residual stress distributions.) All of the following criteria must be met:

- the most severe cycle is within the elastic range of the material:

$$
\bar{\sigma}_{e l, \max }(x, t) \leq\left(\mathrm{K}_{s} S_{y}\right)_{c}+\left(\mathrm{K}_{s} S_{y}\right)_{n c} \quad \text { (R5: Eqn 6.14), }
$$

where $\mathrm{c}$ and nc refer to the creep and non-creep portions of the cycle

- the total fatigue damage using a life fraction rule of the maximum elastic strain range relative to the fatigue limit must be less than 0.05, i.e. $\mathrm{D}_{\mathrm{f}, \mathrm{el}}<0.05$, (R5: Eqn 6.15)

- Stresses do not reset during cyclic loading:

$$
\bar{\sigma}_{e l, \max } \leq \sigma_{r e f}^{R}+\left(\mathrm{K}_{s} S_{y}\right)_{n c} \quad \text { (R5: Eqn 6.14) }
$$

The definitions are in similar in terms of intent, but in contrast to the means of demonstrating the intent, as ASME NH Appendix T A-3 Test requirements, where the A-3 Test requires a satisfying a limitation of creep usage $\leq 0.1$, limitation of creep strain accumulation to less than or equal to $0.2 \%$, and the modified $3 \mathrm{~S}_{\mathrm{m}}$ rule to ensure that stresses do not reset.

If these criteria are not met, then one must continue and follow procedures that address both creep and fatigue failure mechanisms and design criteria.

\subsection{Simplified Inelastic Analysis}

Shakedown can be demonstrated with no specific requirement on the use of numerical or analytical tools. The Code is written specifically to ensure satisfaction of shakedown theory as discussed earlier in terms of elastically calculated stresses and residual stresses. This requires determination of residual stress distribution, the steady cyclic stress state, satisfying shakedown criterion, and ensuring that no more than $20 \%$ of the section wall thickness experiences cyclic plastic loading. Recall, loading in the plastic or ratcheting 
regime is not permitted without conducting inelastic analysis. While certain tools are not dictated, the use of the shakedown reference stress, reference temperature, and the stress at the start of the dwell period is required.

Satisfying shakedown with residual stresses arising from plastic deformation is called 'short-term shakedown'. This is to distinguish it from shakedown and residual stresses that are generated by creep using the effective time and temperature dependent yield strength. Regardless, once shakedown is demonstrated one must demonstrate avoidance of creep-fatigue failure (initiation) and excessive cyclically enhanced deformation due to creep (creep ratcheting).

Creep-fatigue is outside of the scope of this Task. However, a brief summary of R5 is provided in terms of the structural analysis to arrive at the inputs to assess C-F failure, i.e. stress and strain estimates utilized in calculation of C-F damage assessment.

Peak stresses must be considered when assessing creep and fatigue damage. If the peak stresses were not included in the shakedown assessment, a correction is required to arrive at an estimate of the local stress at the start of the dwell period. If peak stresses were included, no correction is required. An elastic follow-up factor is utilized to predict the extent of creep strain increment and its contribution to the total strain range when assessing fatigue damage. Creep damage is assessed with a ductility exhaustion method and criterion, which accounts for strain rate effects and stress state effects on creep ductility. A linear damage rule for C-F interaction is used. If a crack initiates due to C-F, R5 includes procedures to assess crack growth as well; this is the subject of another Task in the DOE-ASME Gen IV Materials Tasks.

While ASME NH Appendix T places specific limits on strain to ensure against cyclically enhanced deformation, R5 does so by incorporating strain limits in its definition of $S_{R}$, $1 \%$ for ferritics and $2 \%$ for austenitics. There is no specific restriction to any strain limits as in ASME NH Appendix T, e.g. 1\% strain averaged through the section of a wall. Rather, R5 implements a creep usage factor, W. Essentially, this is identical to the creep 
usage factor in R5 for primary load limits, with the exception that the shakedown reference stress is used instead of the rupture reference stress.

The use of the shakedown reference stress in this context has the potential for excessive conservatism. If the shakedown reference stress is not consistent with the core stress as explained earlier, then the creep usage factor $\mathrm{W}$ will be overly conservative - the extent of which is dependent upon how conservative the shakedown reference stress is relative to the true core stress in the steady cyclic cycle. Also, as pointed out earlier, the reference temperature is not indicative of the average temperature through the section.

Even if the shakedown reference stress is consistent with the core stress, no specific limitations are given in terms of strain limits as in ASME NH, i.e. there are no $2 \%$ or $5 \%$ strain limits.

As discussed earlier, R5 has no equivalent or alternative simplified procedures for obtaining slow cycle solutions, such as the B-1 \& B-3 Tests in ASME NH Appendix T. There is also no method comparable to the B-2 Test.

\section{Cyclic Margins Against Plastic Collapse:}

The primary load limits in R5 include measures to avoid plastic collapse. Two additional measures against plastic collapse exist that are more closely associated with cyclic loading. These criteria restrict the maximum equivalent stress range, including any contributions from membrane and local membrane stresses as follows:

$\Delta\left(P_{L}+P_{B}+Q\right) \leq 2.0 S_{y}^{\prime}$ for ferritic steels (R5: Eqn 6.3)

$\Delta\left(P_{L}+P_{B}+Q\right) \leq 2.7 S_{y}^{\prime}$ for austenitic steels (R5: Eqn 6.4)

This appears to be a simple limitation on the entire load history stress range (excluding peak stresses) to satisfy a shakedown criteria, where the yield strength is approximately 1.0 and 1.35 times $\mathrm{S}_{\mathrm{y}}$. 


\subsubsection{RCC-MR vs. ASME}

The RCC-MR French Code is very similar to the ASME NH Code in terms of the procedures that it applies to stress and strain classifications, primary load limits, deformation controlled limits, and the use of limit analysis and/or reference stress methods to formulae elastic, shakedown, plasticity and ratcheting analyses.

It appears that the RCC-MR has its basis in the ASME NH Code. The rules in RB 3000 address the design and stress analyses of class 1 equipment to ensure safety margins against mechanical damage ( $\mathrm{P}$ and $\mathrm{S}$ type) under operating conditions and loadings. The "P type damage" results from the application of a steady or constant loading. The S type damage is one caused by cyclic repeated loading. The RB 3000 rules do not address damage resulting from irradiation, erosion or corrosion.

One major difference between ASME NH and RCC-MR is that the latter allows for inelastic analysis to be used for most of the design criteria, including those to be addressed in this Subtask 9.3. The inelastic analyses allowed in RCC-MR are limit and elasto-plastic analyses. NH on the other hand does not permit limit analysis.

\subsubsection{PRIMARY LOAD LIMITS}

\subsection{Elastic Analysis \& Limit Load Analysis}

- Limit Load Collapse under a single load application:

Elastic analysis method for predicting primary load limits and secondary load limits is one of three analysis options that RCC-MR provides, the other two being elasto-plastic and limit analyses which are not allowed by NH Code except for level D loading. RCC MR requires load and stress classification just the same as the ASME Code, and it also requires stress linearization for some of the analysis; stress linearization is handled in the same way as $\mathrm{NH}$. 
Section RB3251.11 addresses elastic analysis, RB3251.12 addresses limit analysis, and RB3251.13 addresses elasto-plastic analysis for negligible creep conditions for level A load. Level C \&D loads are addressed in RB3251.2 \& RB3251.3 respectively. Service load levels affect the factors of safety dictated by these methods. The load limits also are dependent on which analysis method is used.

\section{The elastic analysis}

This method limits the general primary membrane, local primary membrane and primary membrane plus bending stresses to limits that are proportional to Sm. The limits also depend on the geometry of the component, i.e. shell, beam...etc. It is obvious that the elastic method of RCC-MR is identical to that of NH. Just like NH, RCC-MR utilizes the wall average temperature for specifying this design criterion.

\section{The limit analysis}

This method of RCC-MR (RB3251.12) checks if $\mathrm{So} \leq \mathrm{S}_{\mathrm{m}}$, where $\mathrm{S}_{\mathrm{m}}$ is based on the maximum temperature of the structure at wall midsection. Here $S_{o}=\left(C / C_{L}\right) * R_{L}$, where $\mathrm{R}_{\mathrm{L}}$ is the yield strength, $\mathrm{C}$ is the load and $\mathrm{C}_{\mathrm{L}}$ is the collapse load. The latter can be obtained by lower bound theorem or by elastic-perfectly plastic limit analysis. This approach is not allowed in NH.

The elasto-plastic analysis method checks for plastic instability under a load that is 2.5Xdesign load.

\section{Deformation Limited Stress for Time Dependent Failure:}

The primary membrane stress must not exceed the time dependent strength $S_{t}$ (obtained from A3.52). Just like $\mathrm{NH}$, there is no adjustment for $\mathrm{K}_{\mathrm{t}}$ and no interaction of membrane and bending is required; $\mathrm{S}_{\mathrm{t}}$ includes a limit on time to $1 \%$ total strain, or rupture, whichever is lower.

\section{- Excessive Creep Deformation and Rupture Limit for Time Dependent}

\section{Failure:}


For significant creep analysis, limit analysis method must not be combined with any other method. Section RB3252 addresses time dependent criteria with significant creep under P type loading. It states that aging factor $\left(F_{v}\right)$ from A3.51 must be used to satisfy primary load analysis (RB3251). NH Code has a built in time dependent aging factors in the creep rupture data. RCC-MR allows only two methods with significant creep: elastic and limit analysis.

In using elastic analysis:

A. Creep usage factor under primary membrane stress, $\mathrm{P}_{\mathrm{m}}$, must $\leq 1$. A correction factor on the primary membrane stress is identified and is dependent on the level of local primary stress relative to the membrane stress (RB3252.11).

B. In addition to item A, and in the presence of bending stress, the effect of creep is also included, where the creep usage factor is evaluated based on a stress level that is equal to $\mathrm{P}_{\mathrm{L}}+\phi \mathrm{P}_{\mathrm{b}} ; \phi$ is the creep factor (less than 1 ), $\mathrm{P}_{\mathrm{b}}$ is the bending stress, and $\mathrm{P}_{\mathrm{L}}$ is the local stress.

Non-ductile fracture: Fast fracture is not at all addressed in RCC-MR, and there are no design rules to address it. However, the materials approved for design and documented by RCC-MR, are selected with the intent of avoiding fast fracture at the temperature and duration conditions allowed by the code.

Failures of Weldments: RB3100-3200 sections address weldments in terms of strain limits and not deformation limited stresses. Other sections of the Code may address the weld issue by specifying a different value for $S_{t}$, but that is not clear from RB3100-3200 documents.

\section{Multiaxial effects on Creep Failure:}

RCC-MR addresses the effect of triaxiality on creep deformation or creep rupture criteria by replacing the stress tensor $\sigma$ by $0.867 \sigma+0.133 \operatorname{tr}_{j}$, where $\operatorname{tr}_{\mathrm{j}}$ is the trace tensor. 


\subsection{Reference Stress Methods}

RCC-MR allows limit analysis method as an alternative to elastic analysis in P type loading.

\subsubsection{DEFORMATION CONTROLLED LIMITS}

Deformation controlled limits address failure mechanisms that result from secondary stresses, and peak stresses. RB3261 (negligible creep) and RB3262 (significant creep) are sections of the code that address design rules of deformation controlled limits for level A loading. Shakedown is called "Plastic Adaptation”, Reversed plasticity is called “Plastic Accommodation”, and Ratcheting is called “Progressive Deformation”.

\subsection{Elastic Analysis- RB 3261.11 - Negligible Creep}

The elastic analysis is essentially the same as that of NH. It requires the definition of effective primary and secondary stress intensities for cyclic loading (S type). The guidelines for formulating the maximum primary and secondary stress components are well described in RCC-MR. The following factors may be considered which affect the stress levels:

1. membrane secondary stress

2. overstress for short duration (seismic loading)

A correction factor for overstress during short duration is used to modify the maximum primary stress components (RB3261.1).

In order to compute the effective primary stress components, two Secondary ratios are

first computed: $\mathrm{SR}_{1}=\frac{\Delta \mathrm{q}}{\operatorname{Max}\left(\sigma_{\mathrm{m}}\right)}$, and $\mathrm{SR}_{2}=\frac{\Delta \mathrm{q}}{\operatorname{Max}\left(\sigma_{\mathrm{L}}+\sigma_{\mathrm{b}}\right)}$, where $\mathrm{SR}_{1}$ is the ratio of the secondary stress intensity range $(\Delta q)$ to the maximum membrane stress $\operatorname{Max}\left(\sigma_{\mathrm{m}}\right) . \mathrm{SR}_{2}$ is 
the ratio of the secondary stress range $(\Delta q)$ to the sum of maximum bending and local stresses $\operatorname{Max}\left(\sigma_{\mathrm{L}}+\sigma_{\mathrm{b}}\right)$. These two ratios are used to determine efficiency indices $\mathrm{v}_{1}$ and $\mathrm{v}_{2}$, according to a certain procedure that is not justified or explained in the Code. The resulting efficiency is given in Tables Table 9.3.3.2.1 \& Table 9.3.3.2.2.

Table 9.3.3.2.1 Efficiency index

\begin{tabular}{|c|c|c|c|l|l|l|l|l|l|l|}
\hline SR & $<0.46$ & 0.5 & 0.6 & 0.7 & 0.8 & 0.9 & 1 & 1.5 & 2 & 3 \\
\hline $\mathbf{V}$ & 1 & 0.99 & 0.963 & 0.936 & 0.910 & 0.885 & 0.861 & 0.760 & 0.681 & 0.572 \\
\hline
\end{tabular}

Table 9.3.3.2.2 Efficiency index

\begin{tabular}{|c|c|c|c|c|c|c|c|c|c|c|c|}
\hline SR & 4 & 5 & 6 & 7 & 8 & 9 & 10 & 20 & 30 & 40 & 50 \\
\hline $\mathbf{v}$ & 0.5 & 0.45 & 0.41 & 0.38 & 0.35 & 0.33 & 0.32 & 0.22 & 0.18 & 0.16 & 0.14 \\
\hline
\end{tabular}

The effective primary membrane stress intensity $P_{1}$ is equal to the ratio of the maximum of membrane stress $\left(\sigma_{\mathrm{m}}\right)$ to the membrane efficiency index $\mathrm{v}_{1}$ :

$$
\mathrm{P}_{1}=\operatorname{Max}\left(\sigma_{\mathrm{m}}\right) / \mathrm{v}_{1}
$$

The effective primary stress intensity of the sum of primary stresses $\mathrm{P}_{2}$ is equal to the ratio of the stress, $\operatorname{Max}\left(\sigma_{\mathrm{L}}+\sigma_{\mathrm{b}}\right)$ to the efficiency index of the sum of primary stresses $\mathrm{v}_{2}$ :

$$
P_{2}=\operatorname{Max}\left(\sigma_{L}+\sigma_{b}\right) / v_{2}
$$

Limits on P1 and P2 are:

$\mathrm{P}_{1} \leq 1.3 \mathrm{~S}_{\mathrm{m}} \& \mathrm{P}_{2} \leq 1.3 \times 1.5 \mathrm{~S}_{\mathrm{m}}$ for level A load. The factor 1.5 applies to shells and plates; a different factor applies to different components. The first criterion would essentially limit the membrane strain to $1 \%$ (austenitic steels), and the second would limit the strain in the outer fibre to $1.7 \%$.

The second rule is: $\operatorname{Max}\left(\overline{\mathrm{PL}_{\mathrm{L}}+\mathrm{P}_{\mathrm{b}}}\right)+\overline{\Delta \mathrm{Q}} \leq 3 \cdot \mathrm{S}_{\mathrm{m}}$ These limits are identical to those in the ASME-NH code. 


\subsection{Simplified Inelastic Analysis-negligible creep}

RCC-MR recommends two elasto-plastic analyses:

- A validated cyclic elasto-plastic analysis method is available (RB 3233 and A10). Criterion RB 3261.212 is then applied instead of RB 3261.11, which is described in 3.2.1 above.

- Elasto-plastic analysis is used to obtain a realistic classification of the stresses in accordance with the principle given in RB 3261.213.

RB3261.212 criterion contains two sub-criteria:

- Mean plastic strain $\left(\widetilde{\varepsilon}_{\mathrm{m}}\right)_{\mathrm{pl}}$ (RB 3227.6) remains lower than the maximum allowable strain, Dmax, found in (A3.56). Appendix A.356 provides the strain limits based on material type. This is different from $\mathrm{NH}$ in that the strain limits are material dependent.

- Linear plastic strain $\left(\varepsilon_{\mathrm{m}} \tilde{\tau} \varepsilon_{\mathrm{b}}\right)_{\mathrm{pl}}(\mathbf{R B}$ 3227.7) remains less than twice the maximum allowable, $\mathrm{D}_{\max }$, listed in Appendix A3.56.

In determining cycles, one must include an "envelope cycle" which concatenates all cycles. This means that this envelope cycle will assume the highest primary and secondary loads of the group of cycles that it envelopes. NH allows the envelope cycle as an option in Appendix $\mathrm{T}$ for A-tests, and does not require it in B-test. A clear definition of cycles is provided in RB3263.

\section{Deformation Control -Significant Creep}

With significant creep, there are two rules to follow: elastic or inelastic methods.

\section{Elastic Method for Significant Creep:}


The method is very much similar to that of the NH Code in that it relies on definition of effective primary stress intensity which is the membrane stress intensity; the effective membrane stress is corrected by the effect of creep. The secondary stress intensity across the wall thickness (mean value) is also required. The method is based on a Bree type structure. The Code addresses the cases of overstress and provides correction factors for it. The method uses the effective membrane stress and the secondary stress to define two secondary ratios (SR1 and SR2), RB3262.112 as described in the previous section, and these ratios are used to define an efficiency index (RB3262.113). The efficiency index is then used to correct the effective primary stress intensity.

Once the effective primary stress intensities are computed, the following criteria are checked for Level A loading:

- The inelastic strain at 1.25 times the effective primary membrane stress must not exceed $1 \%$-- identical to $\mathrm{NH}$ rule.

- The inelastic strain at 1.25 times the sum of the effective primary stresses (corrected by the effect of creep) must not exceed 2\%-- identical to NH rule.

- The limits in $1 \& 2$ are reduced by a factor of 2 for welds.

\section{Elasto-Visco-Plastic Method with Significant Creep:}

There are two possible methods RCC-MR Code recommends:

1. An elasto-visco-plastic analysis method, which captures the inelastic strains. For this case, the deformation criteria are:

- The mean plasticity plus creep strain (average membrane strain) remains lower than the maximum allowable strain, Dmax given in Appendix A3.56

- The linear plasticity plus creep strain remains less than twice the maximum allowable inelastic strain, Dmax given in Appendix A3.56.

For the elasto-visco-plastic analysis, the initial cycle should consider no residual stresses, but the subsequent cycles will assume the state of stress and strain of the previous cycle. 
2. Elasto-plastic analysis could be used to obtain a more realistic classification of the primary and secondary stresses, as opposed to the elastic approach. Then the resulting primary and secondary stresses will be used to determine the effective stress intensities in a fashion similar to the elastic approach.

As mentioned earlier, the Code makes an attempt at describing how cycles should be defined, and it discusses what it calls an "envelope" cycle and "least favorable cycle". A detailed description of defining cycles and cyclic loads is given in RB3263. However, the description pertaining to complex loading is brief and ambiguous; this may be due to difficulty in translating the Code from French to English.

In general, the procedures and criteria for deformation control are very similar to those in the NH Code. They focus on structures depicted by the Bree tube, and they do not address more complex geometries or loading in any meaningful manner. The RCC-MR Code defines effective membrane stress using efficiency indices obtained by secondary ratios SR1 and SR2 that address the effect of secondary stress on the effective primary stress intensities. The Code does not provide justification to the use of secondary ratios and efficiency indices for predicting effective stresses that drive the elastic approach. It is also unclear if the elastic approach yields the same results as the elastic approach in the NH Code.

\subsubsection{Procedures for analyzing Creep-Fatigue}

\section{Highlights of Procedure:}

1. Time fraction (creep usage) and cycle fraction (fatigue usage) are checked against creep-fatigue interaction diagram.

2. Fatigue analysis is based on strain range as linear sum of elastic, plastic and creep strain ranges:

3. Procedures account for elastic followup for creep strain calculations

Methods used for creep strain prediction: 
1. Elastic Method: For creep strain calculations, the following information and assumptions are made (this is an extremely conservative approach)
a. Highest temperature during hold time is used for creep analysis
b. Highest value of primary stress during hold time is
c. Stress range is identified
d. Primary stress range is identified $\Delta \sigma$
e. Symmetrization coefficient, $\mathrm{K}_{\mathrm{s}}$ is computed (not clear what this factor is)
f. Stress used to compute creep strain is: $\sigma_{\mathrm{k}}=\overline{\mathrm{P}}_{\max }+\mathrm{K}_{\mathrm{s}} \cdot \overline{\Delta \mathrm{S}^{*}}$, where $\Delta \mathrm{S}$ is the secondary stress

A less pessimistic (conservative) elastic approach may be used, and is one that takes into consideration the creep stress relaxation.

The exact procedure of the above is quoted from RCC-MR and given in the section below.

2. Inelastic (elasto-plastic analysis) method may be used to compute the strain range and stress. The stress information is then used to compute the creep strain. The resulting total resulting strain range is then used to predict fatigue damage.

\section{ELASTCIC METHODS: Procedure for computing plastic and creep strain ranges when} holding time exists at one extreme of the cycle. (Quoted from RCC-MR Code)

a. Amplification due to plasticity - Calculation of $\overline{\Delta \varepsilon_{\mathrm{el}+\mathrm{pl}}}$

The "elastic plus plastic" strain range evaluation $\overline{\Delta \varepsilon_{\mathrm{el}+\mathrm{pl}}}$ is evaluated in the same way as $\overline{\Delta \varepsilon}$ defined in the "Amplification due to plasticity - calculation of $\overline{\Delta \varepsilon}$ " of RB 3261.123.

Henceforth, $\overline{\Delta \sigma}$ * will be used to note the ordinate of the point on the cyclic curve (A3.46) for which the abscissa is equal to $\overline{\Delta \varepsilon_{\mathrm{el}+\mathrm{pl}}}$ thus calculated (Fig. RB 3262.123a).

b. Amplification due to creep - Calculation of $\overline{\Delta \varepsilon_{\mathrm{fl}}}$

For each of the cycles to be considered, the following must first be determined at the point examined: 
- the highest temperature during the holding time: $\theta^{*}$

- the period for which, during the holding time, the temperature exceeds the temperature at which the creep effects appear (A3.31) : T*

- the highest value during the holding time of primary stress intensity:

$$
\overline{\mathrm{P}}_{\max }=\operatorname{Max}\left[\overline{\mathrm{P}_{\mathrm{m}}+0,66 \cdot\left(\mathrm{P}_{\mathrm{b}}+\mathrm{P}_{\mathrm{L}}-\mathrm{P}_{\mathrm{m}}\right)}\right]
$$

- the stress range: $\overline{\Delta \sigma}$ *

- the primary stress range : $\overline{\Delta P}\left(\mathrm{RB} 3261.123\right.$ - calculation of $\left.\overline{\Delta \varepsilon_{2}}\right)$

- the secondary stress range: $\overline{\Delta \mathrm{S}^{*}}=\overline{\Delta \sigma^{*}}-\overline{\Delta \mathrm{P}}$

- the symmetrization coefficient $K_{s}$. obtained as a function of the ratio $\overline{\Delta \sigma} * / 2 \cdot\left(R_{\mathrm{p} 0.2}^{t}\left(\theta^{*}\right)\right)_{\min }$ on the basis of the curve given in $\mathbf{A} \mathbf{3 . 4 6}$.

The amplification of the strain range which results from the effects of time (creep) is obtained by using the creep rule (A3.54) to calculate the creep strain due to a stress equal to $\sigma_{\mathrm{K}}=\overline{\mathrm{P}}_{\max }+\mathrm{K}_{\mathrm{S}} \cdot \overline{\Delta \mathrm{S}^{*}}$ held for time $T^{*}$ at temperature $\theta^{*}$. The above formula for calculating $\sigma_{k}$ uses the scalar addition of $\bar{P}_{\max }$ and $\mathrm{K}_{\mathrm{S}} \cdot \overline{\Delta \mathrm{S}^{*}}$. This formula can be extremely unfavourable in a certain number of cases. A better combination of both values can be obtained (Fig. RB 3262.123b) considering the value of $\sigma_{k}$ corresponding, on the reduced cyclic curve, to the sum of strains $\varepsilon_{p}$ (strain for $\overline{\mathrm{P}}_{\max }$ stress) and $\varepsilon_{\mathrm{s}}$ (strain for $\mathrm{K}_{\mathrm{s}} \cdot \overline{\Delta \mathrm{S}{ }^{*}}$ stress).

The Reduced Cyclic Curve is obtained by dividing by 2 the coordinates of the Cyclic Curve (A3.46) corresponding to the highest temperature at the point examined during the cycle considered $(\operatorname{Max} \theta)$.

A less pessimistic value of $\overline{\Delta \varepsilon_{\mathrm{fl}}}$ can be obtained taking into account the relaxation during the holding time of the (scalar) stress noted $\mathrm{s}_{\mathrm{r}}(\mathrm{t})$, whose initial value is equal to $\mathrm{s}_{\mathrm{r}}(0)=\mathrm{K}_{\mathrm{s}} \cdot \overline{\Delta \mathrm{S}^{*}}$. The stress $\mathrm{s}_{\mathrm{r}}(\mathrm{t})$ is limited by a nil minimal value. This relaxation takes place at a speed defined by:

$$
\dot{\mathrm{s}}_{\mathrm{r}}=-\frac{\mathrm{E}}{\mathrm{C}_{\mathrm{r}}} \dot{\varepsilon}_{\mathrm{fl}}\left(\sigma_{\mathrm{k}}\right)
$$

where :

E Young's modulus (A3.22) at the point considered.

$\mathrm{C}_{\mathrm{r}} \quad$ elastic following-up and triaxiality coefficient. This coefficient will be considered as equal to 3 except when the design draughtsman justifies a smaller value.

$\sigma_{\mathrm{k}} \quad$ stress obtained either by adding scalars $\overline{\mathrm{P}}_{\max }$ and $\mathrm{s}_{\mathrm{r}}(\mathrm{t})$, or considering (Fig. RB 3262.123c) the value on the Reduced Cyclic Curve corresponding to the sum of strains $\varepsilon_{\mathrm{p}}$ (strain for stress $\bar{P}_{\max }$ ) and $\varepsilon_{r}$ (strain for stress $\mathrm{s}_{\mathrm{r}}$ )

$\dot{\varepsilon}_{f l}\left(t, \sigma_{k}\right) \quad$ creep strain rate given by the creep strain laws (A3.54) for a stress $\sigma_{k}$ and a creep strain $\varepsilon_{\mathrm{fl}}$, assuming a nil value of this creep strain for $\mathrm{t}=0$.

$\overline{\Delta \varepsilon_{\mathrm{fl}}}$ can then be calculated as follows:

$$
\overline{\Delta \varepsilon_{\mathrm{fl}}}=\int_{0}^{\mathrm{T}} \dot{\varepsilon}_{\mathrm{fl}}\left(\sigma_{\mathrm{k}}\right) \mathrm{dt}
$$

\subsubsection{SUMMARY:}

The following are highlights of the RCC-MR Code Design Criteria for steady state cyclic ratcheting, shakedown and limit load analyses:

1. For the most part, RCC-MR is based on the ASME NH Code 
2. RCC-MR requires stress classification

3. RCC-MR corrects for the effect of creep on membrane stress in the elastic analysis.

4. RCC-MR allows limit analysis for significant creep deformation criteria/rupture for $\mathrm{P}$ type loading, whereas $\mathrm{NH}$ does not allow the use of limit analysis.

5. RCC-MR does not address non-ductile fracture.

6. RCC-MR addresses triaxiality effect on effective stress by adjusting the stress tensor using the trace tensor.

7. RCC-MR uses reference stress method - limit analysis

8. RCC-MR's deformation controlled stress limits are identical to NH's

9. Procedures for obtaining effective stresses in elastic analysis of $S$ type loading are different from $\mathrm{NH}$; they utilize efficiency indices and secondary stress ratios, a process that is not explained or justified. One cannot compare this method to the NH approach or find a common thread so easily.

10. Elastic analyses procedures are essentially limited to Bree tube type component. Procedures are very well documented but lack justification.

11. RCC-MR devotes significant effort to defining cycles (RB3263). However, the description is somewhat ambiguous, possibly due to translation difficulties, and it lacks details that are critical in a complex loading scheme.

12. Procedures for inelastic cyclic analyses are documented in Appendices (A.10 \& A.11). Appendices were not available. 


\subsubsection{MONJU vs ASME}

This comparison is based largely on the Guideline referred to here for brevity as the "Monju” code. This was the original guideline used by Japan in ETD for nuclear plant but has since been superseded by new standards which are not generally available. However, some aspects of the new standards were revealed in two papers by Professor Asada and published posthumously in 2006, which, in addition to describing the work involved in developing the new standards, also provided brief summaries of several innovations relevant to future code development.

In terms of scope, content and procedure, the Monju code is procedurally very similar to ASME NH. For instance, rules governing load categories, stress classification system, deformation limits are virtually identical to NH practice, and definition of design allowables follows the NH format very closely. Implementation of the rules differ in detail; these differences will be discussed below.

\subsubsection{PRIMARY LOAD LIMITS}

\subsection{Elastic Analysis \& Limit Load Analysis}

The Monju guideline relies largely on elastic analysis, the stresses being classified by procedures virtually identical to those adopted in $\mathrm{NH}$. Unlike $\mathrm{NH}$, elastic analysis is not explicitly mandated for design purposes, but no alternative is offered.

The equations for comparing combinations of primary and secondary stresses with design allowables are algebraically and numerically identical to the equivalent equations in $\mathrm{NH}$, with one significant difference. In Monju, short term and long term stresses are distinct stress categories and evaluated differently. In common with $\mathrm{NH}$, primary and secondary stresses are compared separately with time independent allowables, designated by $\mathrm{S}_{\mathrm{m}}$, and time dependent allowables, $\mathrm{S}_{\mathrm{t}}$. However, in Monju, short term stresses are only 
included in the criteria for time independent stresses. Presumably this practice is a result of the special concern in Japan for resistance to seismic loading, a concern which emerges in several parts of the Monju guideline.

No information could be obtained on the procedures used in setting $S_{m}$ and $S_{t}$ limits, since these are described in a separate document which was not available at the time of writing.

\subsection{Reference Stress Methods}

More recently, i.e. in the past 5 years, significant changes have been made in Japanese ETD practice. According to Asada, one of these changes has been to introduce an alternative "assessment without stress classification" based on the Reference Stress concept. This procedure effectively eliminates the category of secondary stress as defined in NH in two stages. The first is to factorize secondary stresses into mechanical induced, discontinuity stresses on the one hand, and thermal stresses on the other. The first subcategory disappears when the Reference Stress approach is adopted for primary load assessment. Thermally induced secondary stresses remain but, as far as can be determined from Asada's paper, they are retained as "total” stresses, and not classified into linear and peak components as is the $\mathrm{NH}$ practice.

1) It appears that some thought has been given in this new approach, to the functions performed by thermal stresses. There are two. The first of these is as a contribution to fatigue and creep/fatigue failure. Both linear and nonlinear parts of the total thermal stresses are required in fatigue assessment, so there is no purpose in factorizing them for this purpose.

2) The second role of thermal stress is in ratcheting due to cyclic operation. Local peak stresses do not play a part in this mechanism and linearization is a useful way of eliminating them as a precursor to shakedown or ratcheting analysis. An alternative way of achieving this end is to ignore local stress or strain concentrations occupying less than some small proportion of the component volume. The Japanese criterion is to ignore concentrations which occupying less 
than $10 \%$ of the wall thickness. This is similar to the R5 practice, but more conservative, as R5 allows a concentration volume of up to $20 \%$ of the wall thickness.

\subsubsection{DEFORMATION CONTROLLED LIMITS}

Deformation controlled limits are presumed to be applicable to the cumulative deformations due to cyclic loading.

The procedures used in Monju are essentially the same as the NH screening tests (T1320) and test B-1 (T-1332) but are not optional, holding equal status with the rest of the guideline. A simplified version of the Bree Diagram given in NH (Fig t-1332-1) is provided in Figure 3.4.1 of Monju without the " $z$ " contours, which are given only in algebraic format.

\subsection{Elastic Analysis}

Deformation limits are the same as those adopted in NH, i.e. 1\% membrane strain, $2 \%$ and bending strain. There is no limit equivalent to the NH 5\% limit on local peak strains

\subsection{Inelastic Analysis}

No specific reference is made in Monju or Asada's papers to deformation limits based on inelastic analysis.

\subsubsection{OTHER DEFORMATION RELATED ISSUES}

\subsection{Elastic Follow-up}

Monju devotes a large amount of time to the problem of “elastic follow-up”. This phenomenon is primarily of concern in the assessment of cyclic damage, but computing elastic follow-up is a structural problem and it is therefore appropriate to address the subject here. 
In $\mathrm{NH}$, elastic follow-up is recognized as a problem in the assignment of stress categories. Thermal stresses are, at least nominally, secondary in character. However, situations arise, such as in the thermal expansion of piping systems, when deformations resulting from thermal expansion can result in local collapse and it can be argued they should be reclassified as primary for the purpose of assessment. $\mathrm{NH}$ offers no specific rules for evaluating elastic follow-up, directing the designer to NH-3250 which in turn calls for whatever analysis is agreed upon with the Owner as part of the specification. Monju states the need to classify thermal stresses as primary or secondary, but then leaves the classification process to “judgment”. In the case of low stress, long term operation, calculations of elastic follow-up strains for simple geometries are provided in an Appendix B.

It appears this is a problem which has been recognized but not yet resolved.

\subsubsection{MATERIAL PROPERTIES}

In common with the ASME Code Monju and is descendents is accompanied by a material database and, presumably, a set of criteria for extracting design allowables from the raw data. Unfortunately no documentation relating to this part of the guideline could be obtained. Therefore it is not possible to comment on it, or compare it with ASME practice. 


\subsubsection{API-579 vs ASME}

\subsubsection{GENERAL COMMENTS ON API 579}

API579 is not a design code and does note parallel Section III in general, or NH in particular, as regards its general methodology. However, as the only ASME standard or code other than $\mathrm{NH}$ addressing elevated temperature operation in detail, it has much to offer in terms of computational methods and assessment procedures, even if these are directed at components already in service.

API 579 is formatted around different types of defects and in this respect, differs from current design practice as addressed in the ASME Code, which does not consider defects of any sort, whether preexisting from manufacture or forming in service. Given the exceedingly long design lives being considered for certain nuclear applications, ignoring the effects of defects may be a policy which might need to be reconsidered in the future. If this occurs, then many of the procedures contained in API 579 will have instant value. The foundation of API 579 is the ASME BPV Code. Basic methods of analysis are virtually word-for-word the same as equivalent sections of the Code, and the same structure of stress classification and application of design allowables is retained.

\subsubsection{DEFINITION OF “ELEVATED TEMPERATURE"}

Elevated temperature operation is generally considered to be operation in the creep range. In reality there is no sharp line delineating the creep range from low temperature operation but, as a practical matter, and to save expenditure of effort performing unnecessary detailed analysis, the common design code practice is to define a material dependent temperature below which no significant creep or creep damage occurs in a typical component lifetime. This is known as the threshold for "negligible creep”. API 579 offers an alternative approach.

Elevated temperature operation is addressed in Part 10 of API 579. In common with other Parts, assessment is prescribed in three Levels. Level 1 is a simple screening level 
intended for use by plant operators as a precursor to possibly more detailed evaluations by experts in FFS.

Level 1 of Part 10 has direct relevance to ETD in that it offers an alternative method of determining the limit for "negligible creep". Unlike the methods current to existing design codes, which seek to define this limit by a fixed temperature, the Level 1 approach used in API 579 uses a simplified graphical procedure based on real material creep and damage data to determine the "negligible creep" threshold on a case specific basis. Success of the method depends on the existence of an appropriate database, which API 579 also provides via access to an extensive material database developed by MPC and published as an integral part of API 579.

Given access to the same database used by API 579, this Level 1 procedure is an effective answer to the problem of whether time dependency needs to be considered or not.

\subsubsection{PRIMARY LOAD LIMITS}

\subsection{Elastic Analysis \& Limit Load Analysis}

Levels 2 and 3 of API 579 address a range of analysis methods from simplified hand calculations, through linear elastic analysis of complex components using FEA, combined with a stress classification procedure identical to that used in the ASME Code, to state-of-the-art applications of material and geometric nonlinear FE computations of complex 3-dimensional structures.

Elastic methods track the procedures used in the ASME Code almost exactly. In addition to the classification methodology given in Section III, API 579 provides specific guidance on implementation of the linearization procedure which is central to stress classification. Several procedures are provided, including a recently developed method which takes a new look at the problem, using attributes of FE modeling. 
Since it is primarily concerned with assessing components which have been in service, possibly for extended periods, and exhibiting various levels of geometric and material damage, the assessment criteria used in API 579 do not correspond exactly with those used in Section II generally, or in NH in particular. The main point of contact, and the one where most can be gained by the comparison, is in the nature of the analyses presented, together with the material properties needed for successful implementation.

Limit analysis is addressed in depth by API 579. Procedures for carrying out limit load analyses and collapse load analyses according to the same definitions contained in Section III of the ASME Code are spelled out in detail.

One difference between design and FFS is that, in the latter case, the load history is known to some degree and is not a matter of conjecture. Therefore, cyclic analysis is considered a more routine operation in API 579 than is normal in the ASME Code generally.

\subsection{Reference Stress Methods}

Reference Stress methods are not currently considered in the evaluation of creep deformations according to Part 10 of API 579. This is because creep deformations per se are not addressed directly in API 579, but only as an interim step toward evaluation of creep and creep/fatigue damage.

\subsubsection{DEFORMATION CONTROLLED LIMITS}

API 579 makes an unstated distinction between deformation limits for the purpose of controlling structural deflections and strain limits aimed at avoiding local ductility exhaustion. This practice avoids the ambiguity in $\mathrm{NH}$, which appears to associate local strain limits with both gross deformation and local ductility exhaustion without distinguishing between the two very different forms of structural behavior.

For the purpose of deformation limits, the same criteria as are contained in $\mathrm{NH}$ are used, i.e. $1 \%$ membrane, $2 \%$ bending and 5\% local. Given that this is a Fitness-for-Service guideline, these “deformation” limits are not mandatory and, presumably, can be 
overridden by the circumstances of the application if these call for more or less stringent limits for functional reasons.

Local strain is limited by a multiaxial ductility criterion based on the equation derived originally by Rice and Tracey, and which defines local ductility in terms of the ductility

of the material in a uniaxial tensile test and the triaxiality factor, $T-\sigma_{E} / o_{\text {mtges }}$. It is in character with the FFS objective that API 579 contains no simplified methods of cyclic analysis equivalent to the Bree diagram or its derivatives. Cyclic analysis, both for the purpose of evaluating ratcheting and creep/fatigue interaction is presumed to be carried out by a full and detailed cyclic FE analysis. Some simplification is allowed in this approach, including the use of cyclic stress/strain curves for cyclic elastic/plastic stress analysis.

Detailed elastic/plastic/creep analyses, whether monotonic or cyclic, require detailed material constitutive models. API 579 provides a reasonably comprehensive database of representative materials for this purpose.

\subsubsection{MATERIAL PROPERTIES}

In line with the generally more detailed analyses demanded of API 579 on a routine basis, this guideline includes a comprehensive database for a representative cross section of alloys used in pressure vessel construction. This database ranges from low carbon steel, through low alloy ferritic steels and austenitic stainless steels to Alloy 800 and HK40. The grouping of these materials is comparable with the groupings used in Section II for the purpose of providing material properties such as Young's Modulus and coefficient of thermal expansion.

The API database is broader in scope than the ASME Code, including equations and material data for brittle fracture, fatigue and creep assessments. 


\subsubsection{REFERENCES}

[1] American Society of Mechanical Engineers, Boiler \& Pressure Vessel Code 2007 Edition.

[2] B. Langer, "Criteria of the ASME Boiler and Pressure Vessel Code for Design by Analysis in Section III and VIII, Division 2”, in Pressure Vessels and Piping: Design and Analysis - A Decade of Progress, Volume 1 - Analysis, ASME, 1970.

[3] T.E. McGreevy, Interim Development of Simplified Methods for Very High Temperature Metallic Design, ORNL/GEN4/LTR-06-016, Oak Ridge National Laboratory, Oak Ridge, TN, July 30, 2006.

[4] T.E. McGreevy et. al., "The Effect of Temperature Dependent Yield Strength on Upper Bounds for Creep Ratcheting”, Proceedings of PVP2006-ICPVT-11, PVP2006-ICPVT11-94011, Vancouver, BC, Canada, July 23-27, 2006.

[5] T.E. McGreevy \& J. Abou-Hanna, “Applicability of Simplified Methods to Alloy 617 in Excess of $649^{\circ} \mathrm{C}^{\prime}$, Proceedings of PVP2007, PVP2007-26672, San Antonio, TX, July 22-26, 2007.

[6] T.E. McGreevy, "Summary of Investigation of Strain Limits in ASME NH Appendix T: A-1 \& A-2 Tests”, Oak Ridge National Laboratory, May 2008.

[7] R L Huddleston, “Assessment of an Improved Multiaxial Strength Theory Based On Creep-Rupture Data for Type 316 Stainless Steel”, ASME J Pres Ves Tech 115, 177-184 (1993).

[8] W.J. O’Donnell \& J.S. Porowski, “Creep Ratcheting Bounds Based on Elastic Core Concept”, Transactions of 5th International SMiRT Vol. L 10/3.

[9] T.E. McGreevy \& J. Abou-Hanna, “A Hybrid Approach to Simplified Design Methods”, Oak Ridge National Laboratory, October 2008. 


\title{
Task 9: Update and Improve Subsection NH - Simplified Elastic and Inelastic Design Analysis Methods
}

\author{
Subtask 9.4 - Review of Modern Methods \\ Developed for Elevated Temperature Design (ETD)
}

Gen IV / NGNP Materials Project

\author{
Submitted to \\ Mr. Jim Ramirez \\ Vice President, Business Development \\ ASME Standards Technology, LLC \\ Three Park Avenue \\ New York, NY 10016 USA \\ By \\ Advanced Consulting Engineering Services, Inc. \\ Peoria, IL \\ On behalf of \\ Jeries Abou-Hanna \\ Douglas L. Marriott \\ Timothy E. McGreevy
}

April 14, 2009 


\section{TABLE OF CONTENTS}

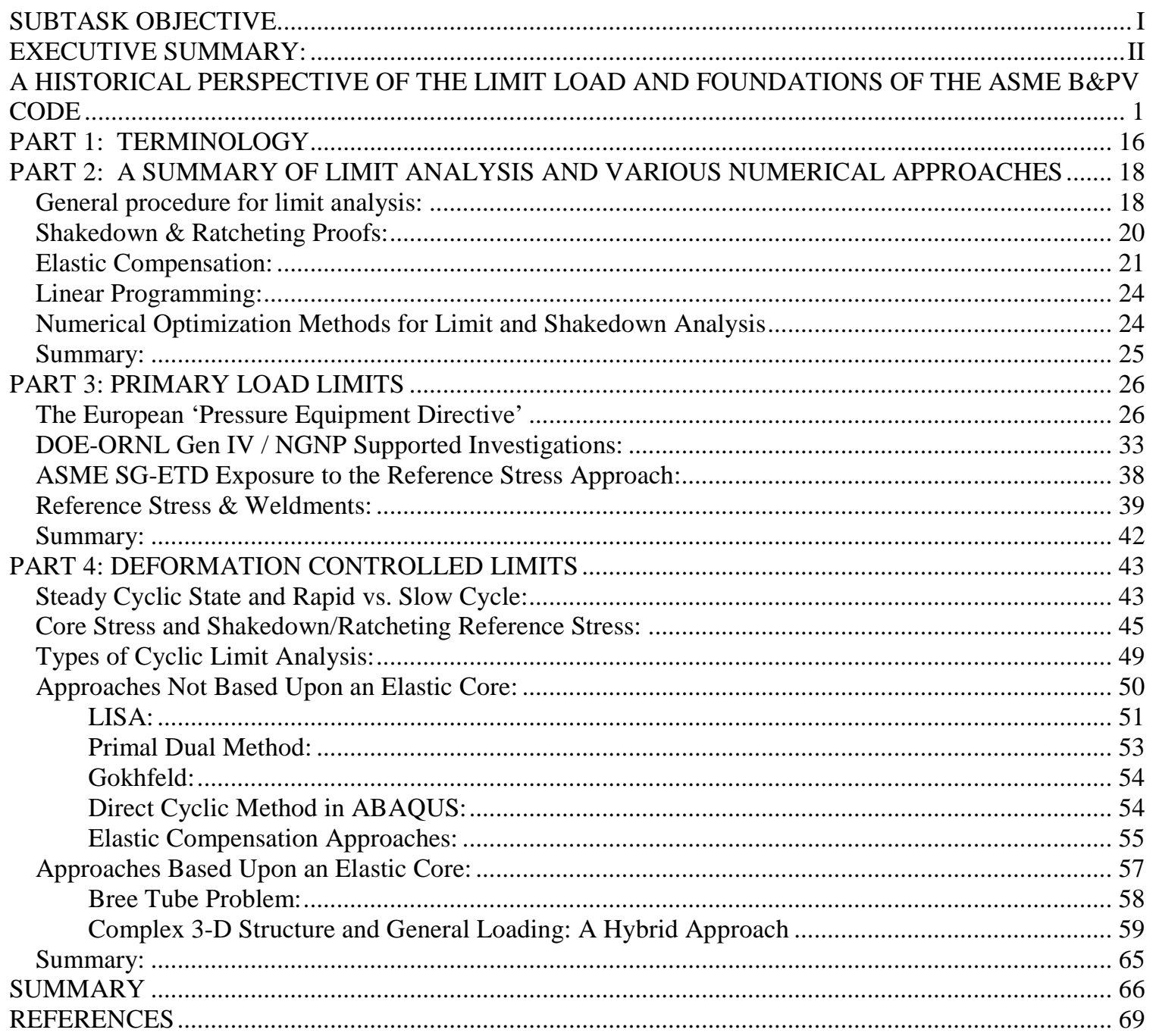




\section{SUBTASK OBJECTIVE}

The objective of this subtask is essentially the same as Task 9.3, but is directed specifically at reviewing new and/or promising methods which may have been published in the open technical literature but which, to date, have not been incorporated into any International ETD Code or guideline. The candidate methods are compared when possible to available applications (geometry \& loading) found in the literature or available to the TI's as a result of their involvement in related R\&D.

As in Task 9.3, where possible, the strengths and weaknesses will be summarized; the scope of these comparisons will be limited to

- Elastic analysis,

- Reference stress methods,

- Limit load, shakedown, and ratcheting analysis.

Note, these three areas are a subset of the Ideal ETD Code and all International ETD Codes. These topics are addressed in terms of Load Controlled Limits (elastic analysis, reference stress methods, and limit analysis) and Deformation Controlled Limits (elastic analysis, reference stress methods, and shakedown and ratcheting analysis). 


\section{EXECUTIVE SUMMARY:}

This executive summary is intended to highlight the key approach and findings of a review of new and/or promising methods which may have been published in the open technical literature but which, to date, have not been incorporated into any International ETD Code or guideline. An exception to this is the reference stress approach as it applies to primary load evaluation, e.g. R5 utilizes this approach extensively. It is included here because of various methods being developed that avoid the need for stress classification. The contents of the report provide more detail in support of this summary. Note, the extent of material in this area is large, with a broad range of application outside of the ASME B\&PV Code current needs. Similarly, the number of various approaches to obtain the same objective - defining the limiting state of a structure or system, is very large.

The use of full inelastic analysis is one option to an analyst or designer; however, the scope of this work is for simplified methods. As such, full inelastic analysis is not addressed herein, i.e. elastic-plastic-creep analysis.

Elastic analysis, as utilized and evaluated in Sections III and VIII/Div.2, reference stress analysis, shakedown, and ratcheting analysis are all variations of the use of 'limit analysis'. Elastic analysis provides a lower bound on the limiting state of a structure, while full inelastic analysis provides a means of a more efficient and accurate estimate of the limiting state of a structure. Note, various inelastic approaches exist that provide lower or upper bounds on inelastic solutions.

Mr. Bernard Langer [1] describes the stress classification methodology adopted by the ASME Code with the introduction of so-called "design by analysis". From his paper, and accompanying Introductions to the Commemorative Volume by Hsu [42] and Cloud [43] it is clear that use of the branch of solid mechanics known as limit analysis had reached maturity in the pressure vessel industry and this development contributed significantly to the basis for the calculation of both primary stresses and for the $\mathrm{P}+\mathrm{Q}$ criterion for elastic 
shakedown in Section III, Part NB, Design of Nuclear Plant. (see also Bernstein [44] for a concise history of the development of the ASME Code). While NB permits elastic analysis for design, this is only in combination with a stress classification procedure which recognizes the mechanisms of failure and assures a lower bound on plastic collapse. As an alternative, NB permits the use of varied forms of elasto-plastic analysis to arrive at a more efficient estimate of the limit load of the structure, or when the complexity of the geometry defies plausible classification of stresses under the simplified method based on elastic analysis.

ETD Code procedures, such as $\mathrm{NH}$, already utilize elastic analysis coupled with stress categorization and linearization approaches, modified in the case of $\mathrm{NH}$ by section factors which increase the level of conservatism, to account for the possible difference between general yielding at low temperatures, and a more localized failure due to creep rupture. In fact, $\mathrm{NH}$ in particular mandates the use of such linear elastic methods for all forms Load Controlled design other than Type D service limits. A significant advantage to the use of modern engineering tools is that even the most complicated geometries and loading can be analyzed systematically and with relative ease eliminating the need to modify elastic analysis to arrive at more efficient limit analysis solutions.

The use of limit analysis for cyclic loading is already in Sections III and VIII/2 of the ASME Code. As described in [1] and [44] the limit on the range of P+Q in Part NB is a direct consequence of the application of limit load principles.

In ASME NH Appendix T, the A \& B Tests are limit analysis solutions in one form or another; the B-Tests address the existence of an 'elastic core', and bounds on the behavior of the elastic core and other portions of the structures, e.g. elastic, shakedown, plasticity, and ratcheting. The process to arrive at these bounds is in fact 'limit analysis'. The ATests are extensions of the limit solutions tempered with engineering judgment and consensus. Similar to the primary load limits in NB, the cyclic limits utilize elastically calculated stresses to obtain a lower bound solution to the limiting state. For example, the A-1 \& A-2 tests only permit loading in the elastic regime; however, the B-Tests 
permit loading in the shakedown, plasticity and even sometimes in the ratcheting regime. In the case of the B-1 \& B-3 Tests, the use of inelastic analysis provided closed form analytical solutions to map elastically calculated stresses to limit analysis results obtained with inelastic analysis; whereas, the B-2 Test was developed through extensive inelastic finite element analysis.

Similarly, elastically calculated stresses in ASME NH are also mapped to limit analysis results, e.g. an elastically calculated primary bending stress is modified by use of a section factor $(\mathrm{K})$ to account for redistribution of stresses during creep based upon the steady state stress distribution in a creeping structure under bending. In fact, redistribution of stresses may result from tertiary creep as well, which such an approach does not consider.

The advancement of computational methods has led to the use of finite element analysis as a standard tool for designers and analysts. Compared with the limitations on computation in existence in 1963 when Section III was introduced, today it is a routine affair in a design environment to construct, mesh and run an inelastic analysis on a threedimensional model composed of several million finite elements in a matter of hours. Such modern inelastic analysis permits one to obtain more accurate solutions to limit states of more complex structures as well. Furthermore, due to changes in education and the availability of tools with this level of computational power, it can be easier, faster and, most importantly, more reliable, for design analysts to perform their computations by detailed methods than by traditional methods based on analytical models. The desire to integrate these modern tools with the ASME design methodology is apparent to many of those currently working in the field of pressure vessel design.

Two approaches may be taken to implementing this technology.

1. One, the seemingly obvious approach, is to resort to detailed elastic-plastic-large deformation FEA for all applications, and this is the route that has been taken in some industries, where inputs such as material properties, are a relatively straightforward procedure. 
2. The other is to utilize advanced computational power in performing simplified analyses, largely to deal with the uncertainties which exist at the design stage in ETD regarding initial knowledge of scantling sizes, material properties and operating conditions. A term coined to describe this approach, and used in several PVP conference sessions is "robust methods" [45], that is to say methods which are relatively insensitive to the quality or quantity of input data and are capable of providing solutions with sufficient accuracy. The classic example of a "robust method" is the reference stress approach, in its original application to estimation of gross structural deformations due to creep. In theory, this task requires only a single creep test performed at the reference stress to predict the general creep induced deformation of the component, once the reference stress for that component under a specified loading is known. Computationally it is as easy to perform a full creep analysis, so computational resources are not the problem. The problem is the lack of knowledge of the precise creep constitutive model, which is seldom known at the design stage. The reference stress technique circumvents this issue and, incidentally, can be proven to give an upper or safe bound on the true creep deformations.

This report addresses the latter, 'robust methods' or 'simplified analysis', which utilizes elastic-plastic analysis of structures for use in ETD.

There is insufficient space in this report to give detailed summary of this vast amount of work in this report. Instead, the authors illustrate the equivalence of many of these methods, and particularly emphasize the extent by which they are dissimilar. Modern limit analysis permits the use of simplified methods in the form of reference stress or core stress solutions.

For load control limits, two illustrative studies of analysis methods to satisfy primary load limits are reviewed: the first with respect to time independent limits, and the second with respect to time dependent limits. Realistic structures and loading comprise the various examples investigated. Application to primary limits of weldments is also noted. 
For deformation controlled limits (e.g. cyclic limits), simple examples are utilized to illustrate how modern approaches can be used in terms of the reference stress approaches (shakedown and ratcheting reference stress approaches) and the concept of an elastic core and core stress. References are cited that illustrate various points, such as how various sources of inelastic deformation, plasticity vs creep, can generate different steady state stresses in the structure; the use of an appropriate steady state stress solution leads to appropriate and often more efficient bounds to creep strain accumulation of the structure. 


\section{A HISTORICAL PERSPECTIVE OF THE LIMIT LOAD AND FOUNDATIONS OF THE ASME B\&PV CODE}

Exact analysis, if it were possible, would require no interpretation. Success or failure of a design would be the end point of the calculations, assuming the material constitutive model was rigorously correct and all geometry and service conditions known with precision.

The fact that this ideal is a practical impossibility for a variety of reasons, means that all design calculations are simplified approximations. They differ only in the degree of approximation, the degree of detail required of the input data and the effort needed to implement them. As approximations they therefore require interpretation in order to provide accurate insight into the real situations being analyzed.

By definition, approximations, simplified or otherwise, are only capable of capturing part of a problem. In mechanical design, when it may be necessary to assure structural integrity against a range of potential failure mechanisms, an equivalent number of partial models may be required, each testing one aspect of the component performance against a specific subset of material properties.

For example, the earliest forms of systematic structural design work, i.e. masonry constructions, were satisfied with the limited objective of ensuring stability. The model, such as it was, consisted of the shape, including location of joints, and location of the resultant of forces inside the structure's perimeter, so as to avoid placing tension on a joint. Material properties were not even a consideration as long as the structure did not try to take tension on a joint in the masonry.

Once the rules relating applied stress to material strength were developed, it was possible to add a quantitative measure of stress versus a strength - based, once again, on equilibrium on critical sections. A great deal of successful design, from the Industrial Revolution until relatively recently, has been based on the simple principle that "equilibrium will be 
satisfied". This is the basis of the long standing "strength-of-materials" approach which is still valid for elements of mechanical design even today.

Sophistication was brought to design by the introduction of linear elasticity. This approach is capable of calculating local stresses with high accuracy and, if one of the criteria of failure depends on the stress at a point reaching some critical value, it can be very useful as an evaluative tool.

However, not all structures are linear elastic, and not all failures depend on reaching a critical stress at a point. Other models and other measures are needed to deal with more complex problems involving participation of the entire structure in the failure mechanism. The truth of this statement has been revealed and amplified very well by the pioneering work done by groups such as the Pressure Vessel Research Council, and reported on in depth by several authors in the B.J. Langer Commemorative Volume of the ASME Decade of Progress [1].

When it comes to design for elevated temperature, the list of potential failure modes increases dramatically, introducing as it does mechanisms related to both time and temperature.

Evaluation of a design concept consists of comparing a predicted characteristic of the component, such as its stress, with a criterion based, usually, on some material limitation, such as yield strength or creep rupture strength.

Traditionally, that is to say up to a century ago, structural design was carried out by very simple procedures, partly because the understanding of material properties was relatively simple and partly because the analytical tools and the engines to drive them were similarly limited.

Before the time of, for example, Timoshenko, mechanical design consisted of simple equilibrium solutions for nominal stress which were compared with equally simple material 
properties, such as the yield strength. Even in Europe, where fundamental elastic solutions were employed to calculate stresses in engineering components, the evaluation was limited to comparing the maximum stress with an allowable value from tests.

This process encouraged a convenient division of labor. On the one hand a designer calculates the stresses produced in his component due to a variety of loads, including thermal gradients. In the meantime other individual tests materials to obtain the quantities needed to set the allowable. This procedure applied to both static and cyclic loading through the adoption of a static strength and a stress/life fatigue curve.

Under this system there is a clear distinction between structures and materials. The structural provides the applied stress. The material test provides the strength. When strength exceeds applied stress, the design is deemed a success.

Shortly before WWII it dawned on research workers in both the USA and the UK that structures made of ductile material did not fail when the maximum stress reached the critical value for the material. For instance, Professor J.F.Baker, later Head of Mechanical Sciences at Cambridge University, discovered, by strain gauging steel structures in service, that stresses far in excess of the values allowed by codes at the time existed in riveted joints - yet the structures remained standing. This led to the development of the so-called "limit" or "plastic" method of structural analysis. This method was first used for the design of the Morrison shelter, a personal protection cage provided in large numbers to Londoners during the air raids in the early years of WWII.

Plastic analysis ignored the largely elastic stresses under operating conditions and, instead, aimed at determining the load at which the structure would collapse. The innovative idea introduced here was the concept of a "collapse mechanism". In a simple beam-and-column structure with several degrees of redundancy, failure would not occur until a sufficient number of "plastic hinges" had formed to convert the structure into a kinematically feasible 
mechanism. Structural integrity is then guaranteed by limiting the service load to some fraction of the limit load.

Besides the paradoxical fact that inelastic analysis actually became easier to perform than linear elastic analysis, this innovation meant that the simple association of strength with stress at a point was no longer valid. With plastic analysis, the entire structure participates in the measure of strength, not just the stress or strain at an isolated point. While this simplifies the design methodology, it now complicates the ability to separate out design criteria into neat piles of material properties and structural stresses.

What follows is an excerpt from the Wikipedia entry about Sir John Baker, the originator of plastic design.

"Steel can either behave elastically or plastically. Elastic deformation is reversible, and with the removal of load the material will return to its original shape, position and stress distribution. Plastic deformation is not reversible, and with the removal of load the material will assume a different shape, position and stress distribution to the one it held originally.

Plasticity theory is based on plastic behavior, and calculates a lower bound on the load that a structure can carry (the load at which it collapses will not be lower than that calculated). This allows a structure to be designed so it will always be able to carry the chosen magnitude of load, even if the exact way it does so is not understood.

Elasticity theory depends on guessing the way in which a structure works, and the loads it will be subjected to, and designing it to carry those loads in the assumed manner. This ensures it is safe if the structure is well understood, but it may not be safe if the structure carries the loads in a different manner. Therefore it gives an upper bound on the collapse load (the load at which it collapses will not be higher than that calculated). Elastic design is sensitive to deformation of the structure, and only works for small deflections. 
During the 1950s Baker and W. Prager of Brown University published a two volume account of the history of steel structures, with plastic theory integral to it. By the 1960s it was being taught in the undergraduate engineering course at Cambridge University. Although plastic theory is theoretically superior to elastic theory, it is still not in common usage today. Elastic theory, in a codified form, is used for the design of most steel structures, mainly due to the relative ease of use of elastic theory and the computerization of the calculations. Plastic theory is used in some structures and specialist applications, specifically in bridge design."

The use of limit analysis, or limit load analysis in structural engineering was recognized in pressure vessel design during the early 1960's and is acknowledged in papers by Langer and others in the 1972 Decade of Progress two Volume set, commemorating Mr. Langer, as having played a significant part in the development of the stress classification methodology - which formed a central part of the new Section III, Design of Nuclear Plant, published in 1962 [45]. By then, as the excerpt above shows, this form of analysis was well established.

The impetus for stress classification came from the realization that, in more detailed design work, all stresses are not equal, and that linear elastic analysis, on its own, did not provide the answers designers were looking for. It is stated several times, by different contributors to the Langer Commemorative Volume $[1,42,43,44]$ that there is a need to associate only parts of the total stress state with certain modes of vessel failure and that the maximum stress obtained from an unfactorized linear elastic analysis would lead to unrealistic and excessively conservative results. First among the concerns for failure of a pressure vessel is the risk of a rupture under internal pressure. By evaluating stresses in thick tubes and notched bars, Langer demonstrated that the only part of the stress state relevant to such a failure mode is the component of stress in equilibrium with the external load or, in this case the pressure - as long as the material has sufficient ductility to allow redistribution of the stresses within the yield criterion without cracking. This thinking, he acknowledges, is pure application of limit load doctrine; specifically application of the Lower Bound theorem of plasticity. 
A second important innovation of the classification system was to recognize the qualified importance of self-equilibrating stresses, such as thermal and discontinuity induced stresses. These only have relevance under cyclic conditions leading potentially to incremental collapse. Here too are found solutions built on limit load concepts. More specifically, concepts of shakedown, or attainment of a steady cyclic state as a result of the formation of a stable residual stress state were acknowledged to be the basis for the $(\mathrm{P}+\mathrm{Q})$ criterion which applies to secondary stresses $[1,45]$.

Finally, peak stress, a critical value identified by unprocessed linear elastic analysis, was shown to have relevance only for assessment of local damage mechanisms.

Although limit load analysis can be simpler to perform than linear elastic analysis, a major reason for adopting the concept was the economy that can be realized by recognizing that a stress state in a loaded structure is made up of two components. One part, the primary stress, is in equilibrium with the external load and is the only part which, due to the lower bound theorem, is required not to exceed the yield strength. In a redundant structure there is an infinite number of possible primary stress states and economy dictates selection of the one with the lowest maximum stress for design purposes. The remainder of the stress state, termed secondary, is self equilibrating and plays no part in determining the limit load.

At the time Section III was introduced there was, however, a significant mismatch between the techniques being used in research organizations and universities for the practical implementation of limit load analysis, and the level of familiarity with this kind of procedure on the part of the rank-and-file design engineer. Fortunately, the contribution of Drucker et al [46] was a set of Limit Load Theorems which enable one to estimate limit loads very easily using sets of consistent assumptions.

A complete limit solution requires the simultaneous discovery of a kinematically admissible mechanism (e.g. by the formation of plastic hinges or zones of plastic 
deformation) and a statically admissible stress state which nowhere exceeds the yield strength of the material.

If a partial solution can be found involving an only kinematically admissible mechanism but not necessarily everywhere in equilibrium or yield, then the solution is an upper bound. On the other hand a statically admissible solution which everywhere satisfies equilibrium without exceeding yield, but not necessarily compatible with a kinematically admissible deformation, provides a lower bound.

This means that any stress state in equilibrium with the applied stresses, if scaled not to exceed the yield strength of the material provides a lower bound on the true collapse load and can therefore be used as a conservative estimate of strength for design purposes.

This is a simple process. In fact it is identical to the simple methods engineers were using before the theory of elasticity was introduced and made everything difficult.

It is ironic that, after so much work went into supposedly raising the technical capabilities of engineers through the introduction of design methods based on methods of elastic analysis, the methods they were using before, often referred to - and sometimes rather scathingly - as "strength-of-materials" methods, turned out, arguably, to be more correct. This has implications for modern code development when venturing to classify methods as "by rule" or by design".

In developing Sections III and VIII/2, it was recognized that structural shapes and load cases would be encountered where simple "strength-of-materials" methods might not be enough for all applications. Simultaneously, computers began to make their mark as a working design tool and provided a basis for carrying out large and complicated calculations in a systematic way which did not depend on individual virtuosity with a slide 
rule. However, at that time, the ability to perform inelastic analyses was limited to the point of being nonexistent and bounding methods did not interface well with the mechanical procedures required of a computer based analysis.

The solution, which solves this problem in part, is to revert, somewhat, to elastic analysis as a means to obtaining a convenient statically determinate stress solution to be used in a lower bound analysis.

An elastic solution, scaled so that the maximum stress does not exceed yield is therefore a lower bound on collapse. Unfortunately it is not a very useful one because it achieves no more than elastic analysis has been able to do for years.

An innovative procedure developed as part of Sections III and VIII/2 was one called "stress linearization". One observed that, in complex components, the maximum elastic stress is frequently a consequence of a nonlinear distribution across a section through the component. In plane stress or strain, or axisymmetric applications the lower bound property can be preserved by replacing stresses on sections by simpler, statically equivalent distributions. The substitution selected for code purposes, although not a necessary restriction, is to use the statically equivalent stresses obtained from Euler beam theory. This section-by-section substitution does not enjoy the benefit of a full section-to-section redistribution. However, this was recognized by Langer [1] who argued that there was some benefit to safety in not admitting full section-to-section redistribution, on the basis that this could lead to excessive inelastic deformations. Nevertheless, such redistribution is allowed under Paragraph NB-3228.1, which permits a simplified form of limit analysis based on the assumption of elastic, perfectly plastic material behavior, as long as a check is made on the deformation accumulation at a value of $2 / 3$ of the limit collapse load.

Stress linearization fails to benefit consistently from the even greater economy possible by separating stresses into primary and secondary categories, because this is a question which 
involves equilibrium of the component as a whole and cannot be evaluated by considering individual sections in a piecewise manner. The result is that, in situations where it is difficult or impossible to classify linearized stresses by some external criterion, the only safe option is to classify it all as primary, thereby incurring a potentially heavy penalty if the linearized stress was even partly secondary in nature. However, it is straightforward to implement as a routine procedure.

The most radical innovation of the Section III/Section VIII/2 methodology was the deliberate separation of total stress into primary (external load bearing) and secondary (self equilibrating residual stresses). Unfortunately no rigorous procedure comparable with stress linearization exists to implement this factorization.

Secondary stress includes all the possible contributions to the formation of an internal self equilibrating residual stress state, including thermal stresses, effects of material processing, interference fits and discontinuity stresses required to force compatibility in redundant structures.

At present the only method available to classify stresses that is supported by ASME Code is a series of rules, based on fundamental understanding of structural mechanics, such as the identification of fixity or discontinuity stresses, or on physics of the loading, as in the case of thermal stresses. This method is referred to in the ASME Code as the "hopper diagram".

Judgment and experience are needed to use the hopper diagram, especially when this is done in conjunction with the linearization process for interpreting stresses obtained from a linear elastic analysis. Since it is based historically on relatively simple geometries, most of which were essentially 2-dimensional, there are further difficulties involved in extending its application to the very complex geometric finite element models in common use today. 
Assuming it is possible to carry out the stress classification process successfully the object is to assign different limits to different components, dependent on the nature of the failure mechanism under consideration.

The two most fundamental of these are the primary stress limit (Load Control Limits) and secondary stress limit (Deformation Controlled Limits). The rest of this report discusses various limit analysis to address both primary and secondary stress limits. 


\section{MARRIAGE OF MODERN ANALYSIS TOOLS WITH FOUNDATIONS OF THE ETD CODE}

Today, the advancement of engineering tools, e.g. finite and boundary element analysis, and their common use throughout industry necessitates the integration of such tools with the basic foundation of the ASME Code.

Let us step back and review the basic need for addressing primary stress limits and secondary stress limits:

- Primary stress limits are intended to ensure against collapse of a structure due to either short term loading event or failure due to sustained long term loading, e.g. creep rupture or excessive deformation/strain.

- Deformation controlled limits, e.g. secondary stress limits, are intended, at least partially, to ensure against excessive deformation of an overall structure due to cyclic loading, e.g. strain limit of $1 \%$ averaged across a section. Other limits related to deformation controlled loading, e.g. creep-fatigue, exist but are not within scope of this report.

In the previous section, the historical perspective of limit load analysis and the classification of stresses as primary or secondary was reviewed. The use of elastic analysis to provide a simplified limit load analysis for structures, and hence, limits to primary loads (stresses) has been very useful for decades, with extension of limitations on time dependent primary stresses for ETD needs.

While not readily apparent, the limits to cyclic loading, e.g. shakedown and ratcheting, fall into the broad category of 'limit analysis'. The primary load limits are actually a subset of the shakedown and ratcheting limit states, e.g. when secondary loads are noncyclic or non-existent. 
Bree developed the limits for various cyclic loading conditions for the classical case of a tube subjected to constant pressure and a cyclic linear thermal gradient through the wall of the tube [2]. In fact, his efforts were an analytical solution to the limit load problem and cyclic load limits (elastic, shakedown, plasticity, and ratcheting).

O'Donnell \& Porowski extended Bree's solution to address the strain limits of the tube problem, specifically the effects of additional creep (enhanced creep) that the structure experiences above and beyond that expected as a result of the pressure load (primary load) alone [3]. This was accomplished with the development of the concept of an 'elastic core'. This 'elastic core' serves as the foundation for several International ETD Codes in addressing Deformation Controlled Limits.

For a variety of reasons, some perhaps warranted and some not, it is much more common place for designers and producers of pressure vessel equipment to design much more complex structures than in decades past. Along with this situation, more complex engineering tools are being utilized in the design and analysis of such structures. Often times, the fundamental knowledge of shell theory, beam theory, etc that was so common place in the past is now being replaced to a large extent by extensive finite element models. What is missing in many cases is an understanding of the impact of various design parameters on the behavior of the structure since the modern analyst is often busy interpreting the extensive output of data from such complex models.

Meanwhile, in part due to advances in the academic world and due to specific efforts to utilize modern analysis methods in international Codes (ETD and non-ETD), a rather broad range of numerical approaches has been developed to address the behavior of structures under primary and secondary loading. These have evolved to approaches such as those proposed by Bree, O'Donnell \& Porowski, Gokhfeld, Ponter, Sheshadri and many others, that in one form or another, are essentially all different versions of 'limit analysis' [2-6]. 
To clarify, limit analysis can be provided in terms of either elastic solutions or inelastic solutions. A wide range of terms are used to describe such approaches, including analytical solutions (elastic or elastic-plastic), direct numerical inelastic analysis (e.g. finite element analysis), or indirect numerical limit solutions (e.g. nonlinear optimization or linear programming methods).

Application of limit analysis to non-ETD, where creep is insignificant, does not require the identification and use of a core stress or reference stress. However, in terms of limit analysis applied to ETD, two major approaches to utilize the results are: the determination of the reference stress (primary reference stress, shakedown reference stress, or ratcheting reference stress), and the core stress.

With that background and introduction, this report aims to accomplish the following in four sections as summarized below:

\section{$\underline{\text { Part 1: }}$}

- Define a set of terminologies for use in the comparison of a wide variety of limit analysis.

\section{$\underline{\text { Part 2: }}$}

- Provide enough background to the topic to convey that limit analysis is supported by proven (undisputable) theory. With such proofs, limit analysis can be solely utilized for application to ETD criteria or tempered appropriately with experience and engineering judgment. In fact, it may even more readily be applied to nonETD - where creep is insignificant. These proofs should provide higher levels of confidence in support of regulators accepting the use of limit analysis in conjunction with various design criteria.

- Convey that there are many different ways to pose problems and as many or more ways to find numerical or analytical solutions to such problems. In the end, with the exception of those approaches that identify and utilize an elastic core region, most approaches are fancy, academic, or perfectly acceptable alternative ways to 
determine either the limit load (primary load limit), the cyclic limit state (elastic, plastic, shakedown or ratcheting boundaries), or the steady state stress (and sometimes strain range) distribution under cyclic loading conditions.

\section{Part 3:}

- Convey that a variety of approaches exist to determine the primary stress in a structure for use in satisfying Primary Load Limit, and that alternative methods exist that do not require use of stress linearization and classification by conventional means, e.g. by use of a 'hopper diagram'.

- Provide a conceptual description of the procedures used to conduct limit analysis for determination of the primary stress (i.e. the primary reference stress).

- Illustrate that the use of classical approaches (stress classifications and design by formula) have been shown to be adequate and efficient for some structures, while nonconservative for others; this is apparently, at least partially, due to how stresses may be redistributed and is also dependent upon structure geometry and loading.

- Advances in application of limit analysis to welded structures have been made, and are used today for the design and construction of structures that operate at elevated temperatures; these approaches incorporate effects of multiaxial stress state, redistribution of stress due to different material strength throughout a weld.

\section{Part 4:}

- Describe what a steady cyclic state of a structure is; provide a definition of a rapid cycle and a slow cycle (solution); describe the role of residual stresses on the steady cyclic state; discuss bounds associated with rapid cycle and slow cycle solutions; define what the terms elastic core / core stress / core strain / and core temperature are, and define the terms cyclic reference stress (shakedown and ratcheting reference stresses),

- Demonstrate how the use of a shakedown reference stress is not the same as a 'core stress', and that the shakedown reference stress is either equal to or more conservative than the 'core stress'. 
- Emphasize the importance of the use of a 'slow cycle' solution approach for structures that operate at elevated temperature, especially those that operate at very high temperatures.

- Note that when assessing Deformation Controlled Limits there are a limited number of such modern simplified methods based upon the concept of an 'elastic core', which serves as the foundation of elastic, shakedown and ratcheting limits in ASME NH Appendix T. Many of the existing tools may be utilized in a manner similar to that of the shakedown reference stress concept in R5.

- Provide a conceptual description of the procedures required in determining the effects of cyclic loading on the core stress and reference stress, and subsequently the core strain for Deformation Controlled Limits.

- Illustrate that shakedown and ratcheting proofs support implementation of practical simplified methods, with various levels of effort/rigor, and for various levels of screening a design (e.g. demonstration of elastic loading, shakedown, plasticity, or ratcheting) in both ETD and non-ETD Code.

The reader is encouraged to read Part 1 as understanding the terminology will be required. However, the reader may wish to skip Part 2 and go directly to Part 3 and Part 4, returning to Part 2 for a more in depth understanding of limit analysis. 


\section{PART 1: TERMINOLOGY}

Below is a brief list of some terms and a simple explanation of their meaning. This is intended to provide the reader with some basic knowledge and can be used to quickly reference as needed.

Elastic Regime: region in an interaction diagram where stresses remain elastic Elastic Core: the last portion of a structure that provides resistance to deformation with incremental amounts of loading, e.g. the last portion of the structure to yield that provides resistance to deformation

Core Stress: the stress in the elastic core region

Core Temperature: the temperature of the elastic core region

Core Strain: the strain level in the region of the elastic core

Limit Analysis: analysis in search of an extreme condition, e.g. maximum permissible load, or minimum unsafe load

Limit Load: the primary load at which a structure collapses

Plasticity Regime: region in an interaction diagram where reversed plasticity somewhere in the structure occurs, but nowhere in the structure is the plasticity not reversed Rapid Cycle: a cycle where no creep takes place (or creep is insignificant relative to plastic deformation)

Ratcheting Reference Stress: a representative stress obtained from cyclic limit analysis that is consistent physically with the meaning of the core stress

Ratcheting Regime: region in an interaction diagram where the structure experiences a plastic ratcheting strain increment

Reference Temperature: the temperature associated with the shakedown or ratcheting reference stress

Shakedown Reference Stress: a representative stress obtained from cyclic limit analysis that is not consistent physically with the meaning of the core stress (more conservative than a core stress) 
Shakedown Regime: region in an interaction diagram where some portion of the structure undergoes inelastic deformation upon initial loading, but thereafter remains elastic

Slow Cycle: a cycle where creep occurs and is significant in terms of its impact on the steady cyclic state

Steady Cyclic State: the steady state condition of a structure subjected to cyclic loading where the same cycle is experienced repeatedly 


\section{PART 2: A SUMMARY OF LIMIT ANALYSIS AND VARIOUS NUMERICAL APPROACHES}

'Limit load analysis' is actually a subset of 'limit analysis'. Simply put, 'limit load analysis' is a common approach that a majority of engineers would utilize to implement 'limit analysis'. 'Limit analysis' may be thought of as the analysis in search of an extreme condition, e.g. maximum permissible load, or minimum unsafe load; this also includes cyclic states such as the shakedown and ratchet limits. The problem involves determining limiting states of variables, e.g. strain and stress states, with constraints imposed by equilibrium, compatibility, yield functions, boundary conditions, and theories such as Drucker's postulate. Limit load analysis is commonly achieved by use of finite element analysis, although this in not required. As discussed in Subtask 9.3, R5 essentially relates the limit load to a 'reference stress' for use in satisfying Primary Load Limits.

Broadly speaking, there are two basic formulations to limit analysis for the shakedown problem: a) those based upon Melan's static shakedown theorem, and b) those based upon Koiter's kinematic shakedown theorem [4]. Melan's provides a lower bound, while Koiter's is an upper bound to the solution. Depending upon the approach taken, either the upper bound or lower bound solution is used. In more advanced numerical approaches today you can formulate the numerical problem in terms of both upper and lower bound solutions; for example, the 'primal dual method' was used to ensure convergence of both upper and lower bound solutions to the 'true solution'. A brief description of a general procedure for limit analysis is given below.

\section{General procedure for limit analysis:}

A general procedure for limit analysis utilized by Gokhfeld is summarized below [4]. Most modern numerically based limit analysis procedures contain very similar steps; for example, Ponter's Linear Matching Method (LMM) [7, 8]. 
1. Discretize the structure such that the following constraints and fields apply to all discretized parts.

2. Define the following constraints in terms of elastically calculated applied stresses (e.g. due to primary and secondary loading) and the to-be-determined residual stresses; also, apply these constraints at extreme points in the cycle (e.g. the extreme points in the simple Bree tube problem are at shutdown and at startup only).

a. Equilibrium constraints on the residual stresses in the interior of the volume: i.e. $\rho_{i j}^{o}=0$, and similar constraints [4]

b. Compatibility constraints: i.e. compatibility equation in terms of strains and displacements.

c. Stress-strain relationships: i.e. flow rule and normality rule.

d. Constraint on closed cycle: i.e. $\rho_{i j}^{o}(x, t)=\rho_{i j}^{o}(x, t+T)$ meaning that the residual stresses at the start of a cycle must be equal to the residual stresses at the end of a cycle.

e. Energy constraints to the closed cycle: i.e. Drucker's postulate.

3. From among the admissible residual stress fields $\rho_{i j}^{o}$ there should be chosen one which makes a functional attain its absolute minimum, under the constraints listed above. See Gokhfeld Chapter 11 for more details [4].

4. The residual stress field and the associated plastic strain rate fields will develop in the steady cyclic state.

5. The residual stress field combined with elastically calculated stress (applied) provides the 'real stress' state. 


\section{Shakedown \& Ratcheting Proofs:}

Analytical solutions essentially require that the same constraints be satisfied, e.g. equilibrium, with the exception of constraints associated with Melan's theorem and the lower bound solution (static constraints), or Koiter's theorem and the upper bound solution (kinematic constraints).

The general approach above applies to both limit load analysis, as well as shakedown and ratcheting analysis. The ratcheting problem requires a bit more proof than the shakedown problem. Gokhfeld developed numerous formulations to the above problem, with various advantages and disadvantages depending upon whether one was looking for only the residual stress field, the entire plastic strain field, etc. He did so for limit load analysis, and both shakedown and ratcheting limit analysis. One of Gokhfeld's significant contributions was the first proof of the ratcheting limit state or solution [4]. He also notes that 'linear programming' can be used to solve certain formulations, while others require nonlinear programming, which was not so advanced at that point in time.

Since Gokhfeld's work, various numerical approaches have been developed in attempt to solve system of equations and inequalities with more efficient means, and for various formulations of the limit load problem, e.g. elastic-plastic material, strain hardening material, temperature dependent yield strength, etc. Elastic compensation methods (various exist) are one approach; other approaches are more 'mathematical' in nature and are based upon various nonlinear optimization techniques. Techniques may vary greatly in terms of their cost to implement, the constitutive equation utilized (elastic-perfectlyplastic, strain hardening), and temperature dependent properties (or not).

Almost all of the limit analysis approaches for cyclic loading fail to address ratcheting, except by default when the shakedown boundary and the ratchet boundary are the same, e.g. for $X \geq 0.5$ in the Bree tube problem. Ponter, recently, does address the entire ratchet boundary - utilizing Gokhfeld's proof, arriving at an excellent procedure to implement - 
the previously mentioned Linear Matching Method (LMM) [7, 8]. In addition, more recently McGreevy and Abou-Hanna developed another approach that also addresses the ratcheting limit - the Hybrid approach [34, 40].

The significant contribution from Gokhfeld was his proof that constant primary loading has no effect on the magnitude of plastic strains (e.g. at a given thermal stress range, increasing the constant primary load does not affect plastic strain ranges) for structures that are not ratcheting; this simplifies terms in the derivation of a ratchet limit state, and permits a solution for the plastic/ratchet boundary to be achieved; no proof of the boundary existed prior to Gokhfeld's work.

As indicated earlier, a large body of work exists with various approaches to solving 'limit analysis' problems. These will be briefly covered or introduced in order to provide the reader with an appreciation for various tools available to solve engineering problems. Then, a summary of several promising approaches, the LMM and the Hybrid Approach, will be provided later in the report.

\section{Elastic Compensation:}

Marriott was perhaps among the first to attempt or utilize a reduction in modulus to solve a nonlinear problem which formed a part of a live design project, at a time when inelastic analysis was not a routine design office analysis tool. In fact, Marriott's motivation was not limit analysis. The problem was motivated by a 'spacer' design issue. The issue was the existence of large linearized stresses in a locality where discrimination between primary and secondary stress was not possible. High bending stresses on a fan around the ends of spacer welds existed, which if one follows ASME procedures, are primary in the hoop direction but possibly secondary in the axial direction. So, the main issue was proper classification of stresses [9].

The decision, in the absence of better guidance, was to rate all stresses as primary, causing endless grief in the design to accommodate the assumed 'primary' stresses. 
Marriott's motive was to devise a method whereby, without recourse to elastic/plastic analysis since elastic finite element analysis was the extent of capability at the time, to come up with a "primary stress" which did not exceed the $\mathrm{S}_{\mathrm{mt}}$ limit. Any stress distribution in equilibrium would suffice; knowing the background to the use of linear elasticity in the ASME Code merely as a means of producing a statically admissible stress state, any linear elastic solution would suffice; including one in which the Young's Modulus was made to vary from point to point. If the high stress of concern was really secondary, the strain would remain sensibly constant if the modulus changed, and the stress would drop.

To implement the concept, Marriott's strategy involved merely reducing the modulus at critical stress locations until the elastically calculated stress intensity dropped below the design allowable, $S_{\mathrm{mt}}$. While finite element models at the time were relatively crude, involving less than 1000 elements, the number of highly stresses elements was less than 10; hence, implementation was feasible, and was only a simple matter to reduce the modulus in any element in which the maximum stress intensity exceeded $S_{\mathrm{mt}}$ and run the problem again.

Upon revisiting the problem sometime later, Marriott realized that the approach was a simple means (at the time) of calculating the limit load. In terms of stress classification, the approach is useful in determining to what extent a thermal stress acted as a primary load as opposed to a secondary load.

Seshadri's GLOSS R-node has received more attention to achieve the same goal [10], with an extension to shakedown analysis by Mackenzie et. al. [11]. Seshadri's GLOSS Rnode method grew out of Marriott's thesis work involving the "Skeletal Point", which revived an idea first put forward by an American engineer in the 1950's, who saw that there is a point within a beam section where initial elastic and final steady state stresses under creeping conditions are equal. Hence, the two stresses could not vary much in the interim and must therefore be reasonably constant. Given the kinematic constraint of an Euler beam, a creep test at this "skeletal point" stress gives the creep deformation of the 
beam. Marriott attempted to extend this concept to more complex structures. Seshadri pursued "skeletal points" as being nodes of zero stress change, resulting in the R-node concept; the relation between reference stress, limit loads and ASME stress classification was discussed by Seshadri and Marriott [12].

The same concept has been investigated and used by the Japanese in conceptual design efforts for Fast Breeder Reactors [13]. They adopt the concept of Seshadri-s R-node, and incorporate inelastic analysis directly rather than indirectly by use of the elastic compensation approach. Their approach requires the use of a very small hardening coefficient and a fictitious small yield stress in order to properly identify the R-node (Redistribution node). While successful, trial and error were required to set the level of hardening; also, the approach is sensitive to the fictitious yield stress used relative to the level of external primary load (stress). A more robust method would likely be preferred, but the ability to identify the primary stress without section by section evaluation and without stress classification is supported by the approach.

Ponter's method is one of the most advanced of the elastic compensation approaches; with it one is capable of conducting both limit load analysis to find the primary reference stress (or simply 'reference stress'), as well as 'shakedown' and 'ratcheting' reference stresses and boundaries for cyclic analysis [5, 7, 8]. Ponter et al formulated Gokhfeld's proof in a manner that could be implemented with elastic compensation methods while utilizing a popular commercial finite element code, Abaqus, as a solver; this permitted them to obtain the entire stress and strain field for the steady cyclic state at extreme points (points of interest) in the cycle. The use of Abaqus not only provided them with a solver, but its pre-processor, file handling, results viewer, etc. were also valuable in constructing finite element models. The LMM is an effective limit analysis tool in that regard.

In fact, Ponter et al. have recently extended the approach to determine the steady cyclic state of a structure under arbitrary loading and arbitrary time/dwell conditions; some may consider this less of simplified method and more closely resembling a more efficient 
finite element approach; depending upon how it is implemented, it is still a simplified method [14-16].

\section{Linear Programming:}

Gokhfeld was a pioneer in limit analysis of structures, particularly for cyclic loading applications [4]. Many of his formulations can be solved with 'linear programming' techniques; these are problem formulations where the equations and/or inequalities are linear functions of the unknowns. In addition, many of his formulations are also of the non-linear type. Gokhfeld did not expand upon the numerical techniques to solve such formulations; rather he focused on the concepts and theories to prove the existence of shakedown and ratcheting, with most example problems being solved analytically or by hand, or simplified to the extent that the limited computer capabilities at the time could be used to illustrate such concepts.

Extensive knowledge exists today with regards to linear programming techniques, and is not reviewed further herein.

\section{Numerical Optimization Methods for Limit and Shakedown Analysis}

Various formulations of limit analysis may result in functions and inequalities that contain non-linear relationships with unknowns such as strain and stress, e.g. many of Gokhfeld's formulations. These require more sophisticated numerical methods to find the limit state than linear programming techniques.

Use of conventional finite element analysis is typically termed a 'direct route' as it essentially is direct simulation of material and structural response through increments in time as loads are incrementally applied. The limit state may also be determined by an 'indirect route', where the limit state is iteratively achieved by optimization techniques; these optimization techniques typically do not involve marching through time. While 
'linear programming' falls into the category of the 'indirect route', many of the modern indirect approaches require non-linear optimization.

In one project, a variety of approaches have been investigated and comparisons made by applying to a variety of practical pressure vessel structures and loading conditions. The project was called LISA (Limit and Shakedown Analysis) and was for industrial use in the earlier 1990's and lasted for about 10 years [17]. It was supported by The Commission of the European Communities. A summary of the results is provided later; details with regards to implementing the various non-linear optimization techniques are outside of the scope of this report.

\section{Summary:}

Shakedown and ratcheting proofs provide a solid foundation for application of limit analysis in design; limit load analysis is a subset of limit analysis. A wide range of techniques exist to arrive at limit analysis solutions. A limited number of such approaches exists that address the ratcheting limit state, while a large number of approaches exist that address the shakedown limit and primary limit load conditions. 


\section{PART 3: PRIMARY LOAD LIMITS}

The use of Limit Analysis in ETD to satisfy Primary Load Limits is very well established, more so than its use for Deformation Controlled Limits. There is an immense number of documented applications of similar approaches to the design of pressure vessels. Following is a summary of two extensive studies that illustrate very effectively the use of Limit Analysis for Primary Load Limits, and the advantages that come along with its use.

Specifically, the two studies illustrate the advantages and disadvantages of conventional methods such as Design by Formula (DBF) and Stress Classification (SC) that are used in many International Codes, and the pro's/con's for both 'direct route' and 'indirect route' limit analysis solution methods.

\section{The European 'Pressure Equipment Directive'}

An excellent summary of Design by Analysis approaches was conducted for the European Commission's Directorate General for Industry to promote the proposed Pressure Equipment Directive, and particularly the draft unfired pressure vessel European standards. The commission notes that computational power and economic factors were primarily responsible for the attention to the modern methods which require more detailed investigation of pressure equipment. At the time, the draft CEN unfired pressure vessel standard prEN 13445-3 permits design by analysis options by direct routes, with the intent to overcome difficulties associated with stress categorization for which many Codes are based. The authors were unable to determine to what extent the draft CEN prEN 13445-3 standard was accepted and approved, but adoption of the draft was confirmed in a recent ASME SG-ETD meeting. 
The Joint Research Centre Institute for Advanced Materials in Petten, Netherlands received the contract from the European Commission. Key technical roles were conducted by members of the European Pressure Equipment Research Council (EPERC). Ten example cases covering a wide range of component geometries and loading conditions were examined and published in a Design by Analysis manual [18]. Eight of the ten examples included comparative predictions of limit states with respect to gross plastic deformation (addressing failure modes of ductile rupture and excessive local strains), while some limited cases addressed progressive plastic deformation (shakedown / plasticity / ratcheting). Many of the cases addressed cyclic fatigue design checks, and are not discussed herein. These ten examples are:

1. Thick unwelded flat head

2. Thin unwelded flat head

3. Welded-in flat end without nozzle

4. Welded-in flat end with nozzle

5. Storage tank (cone-cylinder junctions)

6. Thin-walled cylinder-cylinder intersection

7. Thick-walled cylinder-cylinder intersection

8. Dish end with nozzle in knuckle region

9. Nozzle in spherical end with cold medium injection

10. Jacketed vessel with jacket on cylindrical shell only, and flat annual end plates

While these examples do not operate in the creep regime, they serve as excellent examples of how to address issues related to stress classification, and satisfying primary load limits. Of particular value is a section that summarizes the difficulties in implementing stress linearization and categorization, including the use of shell discontinuity analysis. The work also points out the use of 'Limit Analysis' in ASME VIII Div 2 Appendix 4-136.3; and it summarizes the use of 'Plastic Analysis' in ASME VIII Div 2 Appendix 4-136.5, which ultimately utilizes the 'twice elastic slope criterion' as used in experimental analysis. 
Several different 'plastic design loads' may be defined: limit load, plastic collapse load, and the ultimate load.

A 'limit load' is one where the load is increased to an extent that overall plastic deformation of the vessel occurs. The strain and displacement are of the small deformation theory type, and the material is either rigid plastic or elastic-perfectlyplastic.

The 'plastic collapse load' utilizes actual strain hardening material properties and large deformation theory to incorporate geometry changes during loading. At the plastic collapse load the structure plastically deforms overall. This would constitute 'failure' of the structure, although larger deformations may be possible before the structure may actually collapse.

For comparison, the limit load for an idealized structure is typically assumed to occur when the structure is largely plastic under small deformation theory - this is typically assumed to be equivalent to the plastic collapse load of a vessel or structure.

The 'ultimate load' is the load for which the vessel actually collapses; the vessel may not actually collapse at the 'plastic collapse load' since strain hardening is included in the material model.

Since the effects of strain hardening will increase the load carry capacity of a structure, a rigid plastic or elastic-perfectly-plastic material is recommended. Geometry changes may strengthen or weaken the structure, depending upon the structure of interest. Furthermore, elastic constraint may restrict plastic deformation to very localized regions and form hinges where large amounts of strain may occur.

To address these issues, the following recommendations were made within the Design By Analysis Manual: a) use of proportional increase in all actions (pressure and thermal) in determining the limit load, b) an elastic-perfectly-plastic material (or rigid plastic), c) 
small deformation theory, d) Tresca yield criterion with associated flow, and e) some specified design strength parameters chosen from simple structures and pressure action to force DBA and DBF to agree. Upon determination of the limit load, the limit load shall be defined as lower of the following: i) the load at which the limit load is reached, ii) the load at which the (absolute) maximum principal strain is $5 \%$.

In comparison with R5, R5 instructs that only mechanical loads should be applied, with the exception that long range thermal loads be applied as mechanical loads.

The use of Tresca yield criterion is deemed by the authors to be unnecessary. Also, introducing thermal loads that must be ramped indefinitely until collapse makes no sense; and to do so in terms of equivalent applied mechanical loads brings one full-circle to one main issue - avoiding stress linearization and classification.

Including the temperature field in the limit analysis in conjunction with a temperature dependent yield strength to account for the effects of spatially varying strength on the limit load is justifiable.

To summarize the findings in eight of the ten examples evaluated, conventional approaches were conducted with design by formulae (DBF) or design by analysis using stress categorization approaches (SC). These or similar approaches are found throughout ASME Code and other International Codes (nuclear and non-nuclear). Modern approaches including design by analysis using direct route elasto-plastic approaches with the aid of commercial finite element codes with small displacement theory (DRS), design by analysis using a direct route elastic compensation approach (DRC), and design by analysis with the elasto-plastic approach plus nonlinear geometry option (NLG) were also conducted.

Table 1 summarizes the findings in the study. The type of analysis conducted is listed in the first column, as defined above. The problem type is listed in the first row. The subsequent values in all other cells are the permissible pressures in units of MPa. Where 
multiple numbers are provided in a given cell, the numbers represent independent analysis conducted by participants, and often included use of different mesh and software (ANSYS vs. ABAQUS). Note, some analysis was conducted with Tresca yield criterion, others with Von Mises. When Von Mises was used, the permissible pressure was scaled by $\sqrt{3} / 2$. Also, the partial safety factors recommended by the study were used, e.g. 1.2 on pressure, and 1.25 on resistance. These partial safety factors were calibrated with respect to design by formulae (DBF) results for simple problems in an attempt to have consistent comparison for various approaches relative to DBF.

A color code was utilized to highlight some trends in the table. All comparisons were made with respect to the direct inelastic route approaches (DRS and NLG). The DBF (design by formulae) predictions are often excessively conservative (green color), with one case being excessively un-conservative (red color for the Dish end with nozzle in knuckle). The SC (stress classification) approach is typically consistent with DRS and NLG, with two exceptions - overly conservative for the Dish end with nozzle in knuckle, and un-conservative for the thin-walled cylinder to cylinder junction. The elastic compensation approach (DRC) is often times excessively conservative (green colors) and marginally conservative (light blue); however, for the thin-walled cylinder to cylinder problem it is nonconservative. For this case, it is noted that the elastic compensation approach used was incapable of considering the 5\% local strain limit in determining the permissible pressure; this does not preclude other elastic compensation approaches from being able to do so.

In summary, for the most part the use of design by formulae (DBF) approaches is overly conservative, with the exception of one un-conservative case. The use of stress classification (SC), while difficult to implement, was consistent with direct route approaches with only one overly conservative and un-conservative case. The elastic compensation method (DRC) utilized was equally conservative as design by formulae (DBF), with an un-conservative prediction for the same un-conservative case as stress classification. 
The study also included recommendations for sufficient Continuing Professional Development Courses in the area of finite element, elastic-plastic theory, constitutive equations, and plate and shell theory. The reason was related to concerns that designers should not utilize such approaches as a 'black box'. The authors may as easily make the statement that implementation of design by formulae (DBF) approaches used in Codes should have the same requirement.

In comparison to $\mathrm{R} 5, \mathrm{R} 5$ permits use of the elastic compensation methods, noting the need for both upper and lower bound solutions to obtain more accurate solutions. R5 also recommends finite element analysis using elastic-perfectly-plastic material with small deformation theory - utilizing the results as a lower bound when the analysis no longer remains convergent. R5 also permits use of literature solutions for specific geometries and loading, and of course experiments. Finally, R5 also permits approximations of limit loads by inversion of Design Codes, for example, in pipe-work and boiler header components. 
Table 1 - Summary of DBA Gross Plastic Distortion Limits (Primary Load Limits) [18]

\begin{tabular}{|c|c|c|c|c|c|c|c|c|}
\hline $\begin{array}{c}\text { Type of } \\
\text { Analysis }\end{array}$ & $\begin{array}{c}\text { Thick } \\
\text { unwelded } \\
\text { flat head }\end{array}$ & $\begin{array}{c}\text { Thin } \\
\text { unwelded } \\
\text { flat head }\end{array}$ & $\begin{array}{c}\text { Welded-in } \\
\text { flat end } \\
\text { without } \\
\text { nozzle }\end{array}$ & $\begin{array}{c}\text { Welded-in } \\
\text { flat end } \\
\text { with nozzle }\end{array}$ & $\begin{array}{c}\text { Thin-walled } \\
\text { cyl-cyl }\end{array}$ & $\begin{array}{c}\text { Thick- } \\
\text { walled cyl- } \\
\text { cyl }\end{array}$ & $\begin{array}{c}\text { Dish end } \\
\text { with nozzle } \\
\text { in knuckle }\end{array}$ & $\begin{array}{c}\text { Nozzle in } \\
\text { spherical } \\
\text { end }\end{array}$ \\
\hline DBF & 17 & 4.2 & 12.7 & 7.8 & 0.28 & 14 & 0.58 & 13 \\
\hline SC & 69,58 & 5.6 & 12.3 & 10 & 0.45 & 14.2 & 0.26 & $\mathrm{~N}-\mathrm{A}$ \\
\hline DRS & $60,58,62$ & $5.7,6.1,5.7$ & $\begin{array}{c}12.6,12.4, \\
12.4\end{array}$ & $9.9,10,9.9$ & $0.39,0.38$ & $14.7,15.1$ & $0.37,0.42$ & $13,13.1$, \\
\hline DRC & 62 & 4.5 & 10.8 & 8.4 & 0.46 & 11.5 & 0.41 & 11.1 \\
\hline NLG & 58 & 5.6 & 12.4 & 9.9 & NC & NC & 0.42 & N-A \\
\hline
\end{tabular}

Gross Plastic Distortion Limit (MPa); NC=not conducted; N-A: no pressure allowed due to magnitude of thermal stresses according to conditions in the SC route.

excessively conservative

excessively nonconservative

marginally conservative
DBF: Design by Formula

SC: Stress Classification

DRS: Direct Route - Small Displacement Theory

DRC: Elastic Compensation Method

NLG: Direct Route - Non-linear Geometry 


\section{DOE-ORNL Gen IV / NGNP Supported Investigations:}

Efforts to gain support of utilizing reference stress (i.e. limit load analysis) to eliminate issues with classification of stresses, were supported by DOE and ORNL in 2005 and reported in [19]. The assessment of load controlled stress design criteria was included. A brief summary is provided below.

All structures examined were assumed to be made of Alloy 617, and were at $900^{\circ} \mathrm{C}$. Several types of modeling approaches were utilized. The first was the use of a simplified version of the Omega model for Alloy 617, which in essence captures deformation due to tertiary creep; explicit modeling of 'material failure' due to creep was not included. Rather, excessive deformation of the structure was used to constitute 'failure' or 'rupture'. Second, the Omega model was used to construct isochronous curves for various times. Note, these curves were generated with the assumption of constant strain rate rather than constant stress or load. The third approach utilized was that consistent with R5's analysis using a reference stress calculation. Finally, the two ASME NH methods permitted were utilized: the first simply based upon $\mathrm{P}_{\mathrm{L}}+\mathrm{P}_{\mathrm{B}}$ and the second based upon $\mathrm{P}_{\mathrm{L}}+\mathrm{P}_{\mathrm{B}} / \mathrm{K}_{\mathrm{t}}$. All loading conditions were selected such that the reference stress was $20 \mathrm{MPa}$, resulting in reference stress creep life predictions being equal for all structures examined.

The following structures were analyzed: 1) a deep notched tensile bar, termed a "yoyo", that was loaded in tension in the longitudinal direction, 2) a plane strain double edge notched bar in bending, essentially the "yoyo" geometry in bending, 3) a plane strain ligament, termed "ligament", loaded longitudinally in tension 4) an axisymmetric nozzle under internal pressure, 5) a 3-dimensional cylinder to cylinder nozzle under internal pressure, 6) fixed end/edge plane stress beam, circular and square plates under uniformly distributed loading, and 7) flathead closures with varying ratios of tube to end wall thickness under uniformly distributed loading. Figures 1-3 illustrate these models. Representations of several of the finite element models and meshes are illustrated in Figure 4. 
The notched specimen geometries were analyzed because they represent structures with large amounts of hydrostatic tension. The nozzle and cylinder models were analyzed due to their complex tube structures, which incorporate notches and 3-dimensional effects. Beam and plate structures were also analyzed to investigate further implications of 3dimensional effects, and the redistribution of stresses under creep conditions.

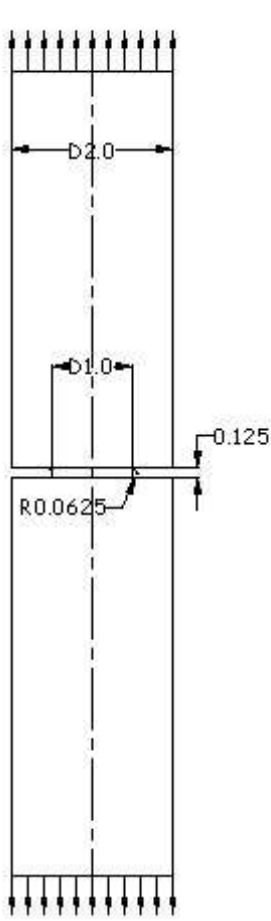

axisymmetric "yoyo" notch

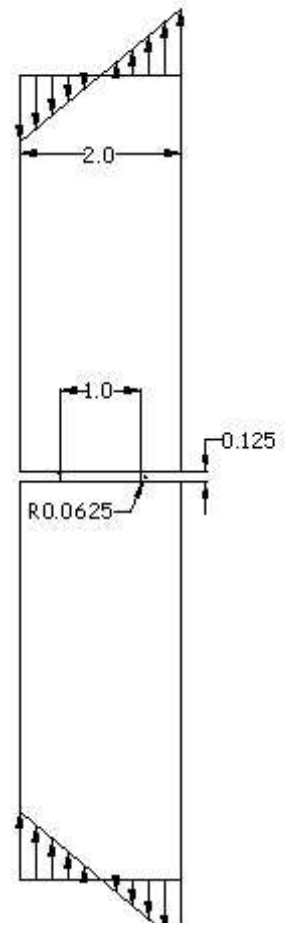

plane strain notch in bending

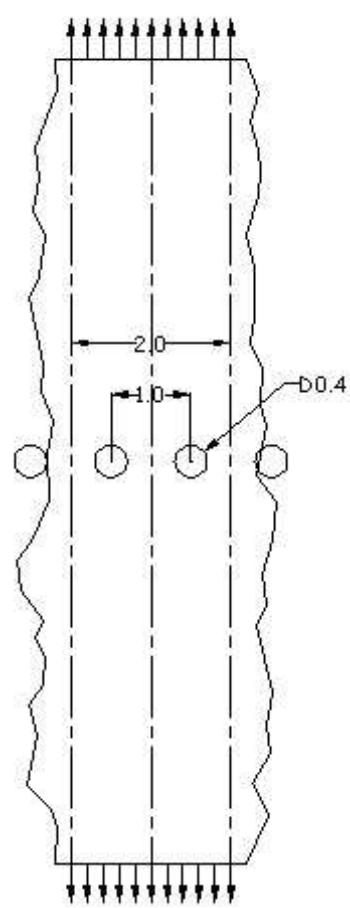

plane strain ligament in tension

Figure 1 Dimensions (inches) of several simply notched structures [19] 


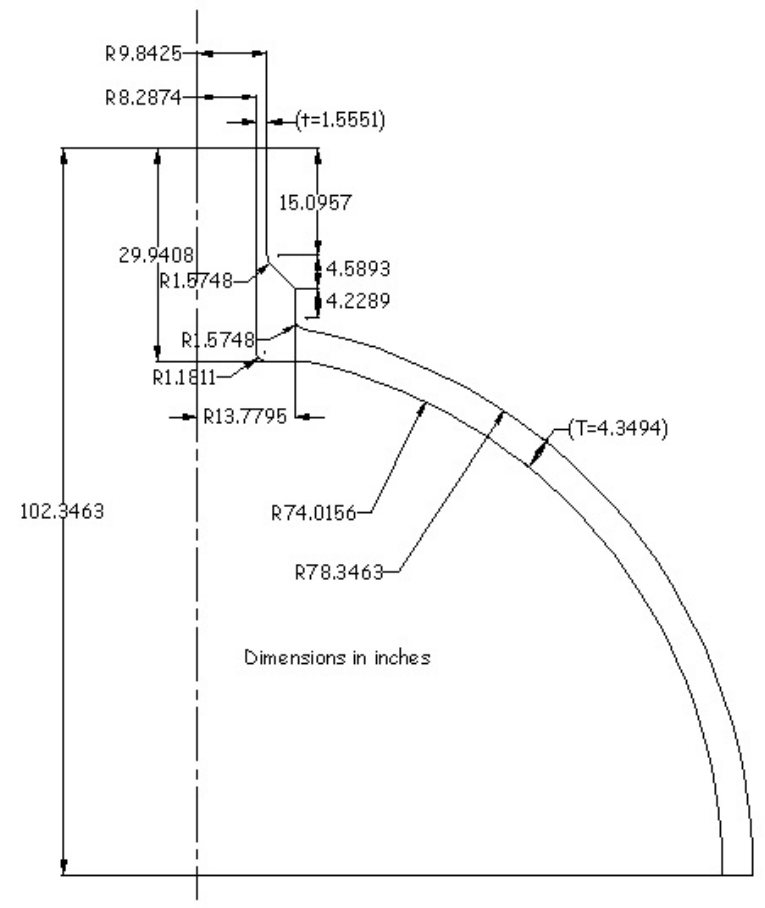

Figure 2 Dimensions (inches) and cross-section of sphere/nozzle and cylinder/nozzle intersection [19]

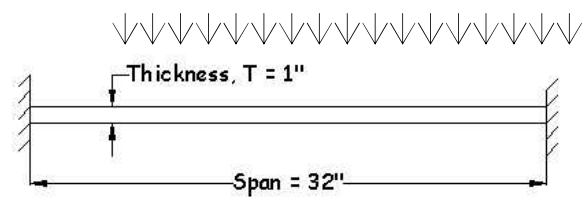

FIXED END, PLANE STRESS BE AM

FIXED EDGE CIRCULAR PLATE

FIXED EDGE SQUARE PLATE

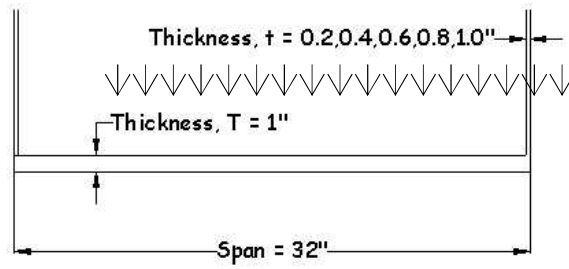

FLATHEAD CLOSURE

Figure 3 Beams, plates, and flathead structures with uniformly distributed loads were investigated (dimensions in inches) [19] 


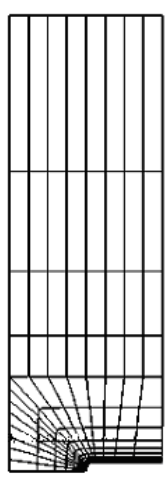

"yoyo"

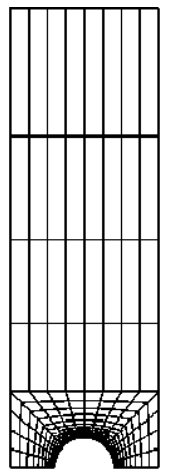

ligament

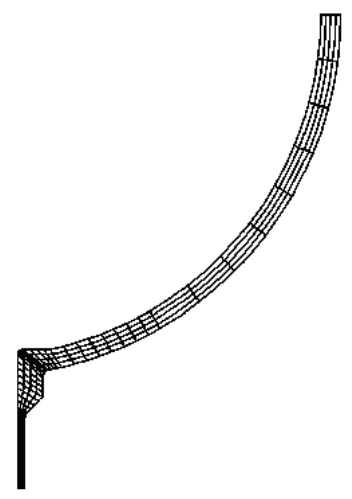

axisymmetric nozzle

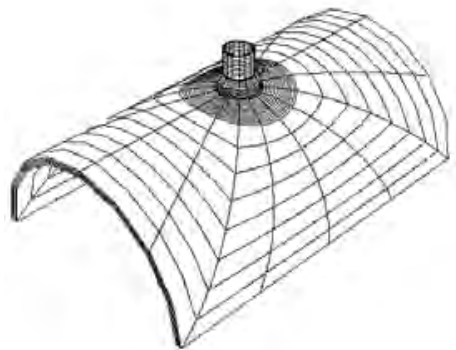

cylinder to cylinder

\section{Figure 4 Several illustrations of finite element models and meshes for notched bars and structures that were analyzed [19]}

Life predictions based on the Omega model, $\mathrm{t}_{\text {omega }}$, were considered as the best approximations to the true solution. The other methods are compared to this "true" result, the reference stress approach, isochronous curves based on the Omega model and constant strain rate, and the two NH load based design procedures.

The objective of the study was to assess the applicability of $\mathrm{NH}$ to very high temperature materials, with the expectation that $\mathrm{NH}$ would be found to be overly conservative. As can be observed in Figure 5, this is not always the case. The predicted creep life for fixed reference stress is plotted for notched specimens, pressure vessel components, plates, and beams analyzed. Both the reference stress and the isochronous stress-strain methods track the Omega solution closely. The reference stress tends to consistently overestimate the creep life by a factor of about 2 , while the isochronous stress-strain method underestimates life by a factor of 2 to 3 .

However, the ASME-NH procedures were found to be inconsistent. NH based life predictions for the notched specimens were comparable with the detailed Omega solutions, with the exception of the double notched bend sample. The cylinder/cylinder nozzle case revealed the $\mathrm{NH}$ procedures to be very pessimistic. The reason for this 
difference in performance was believed to be due to the influence of bending. Another possible explanation was the way stress is redistributed during creep [20]. Stress redistribution during creep can occur both within sections and from one section to another. The ASME procedure only deals with relaxation within a section; no allowance is included for the possibility that section bending and membrane forces also undergo long range relaxation.

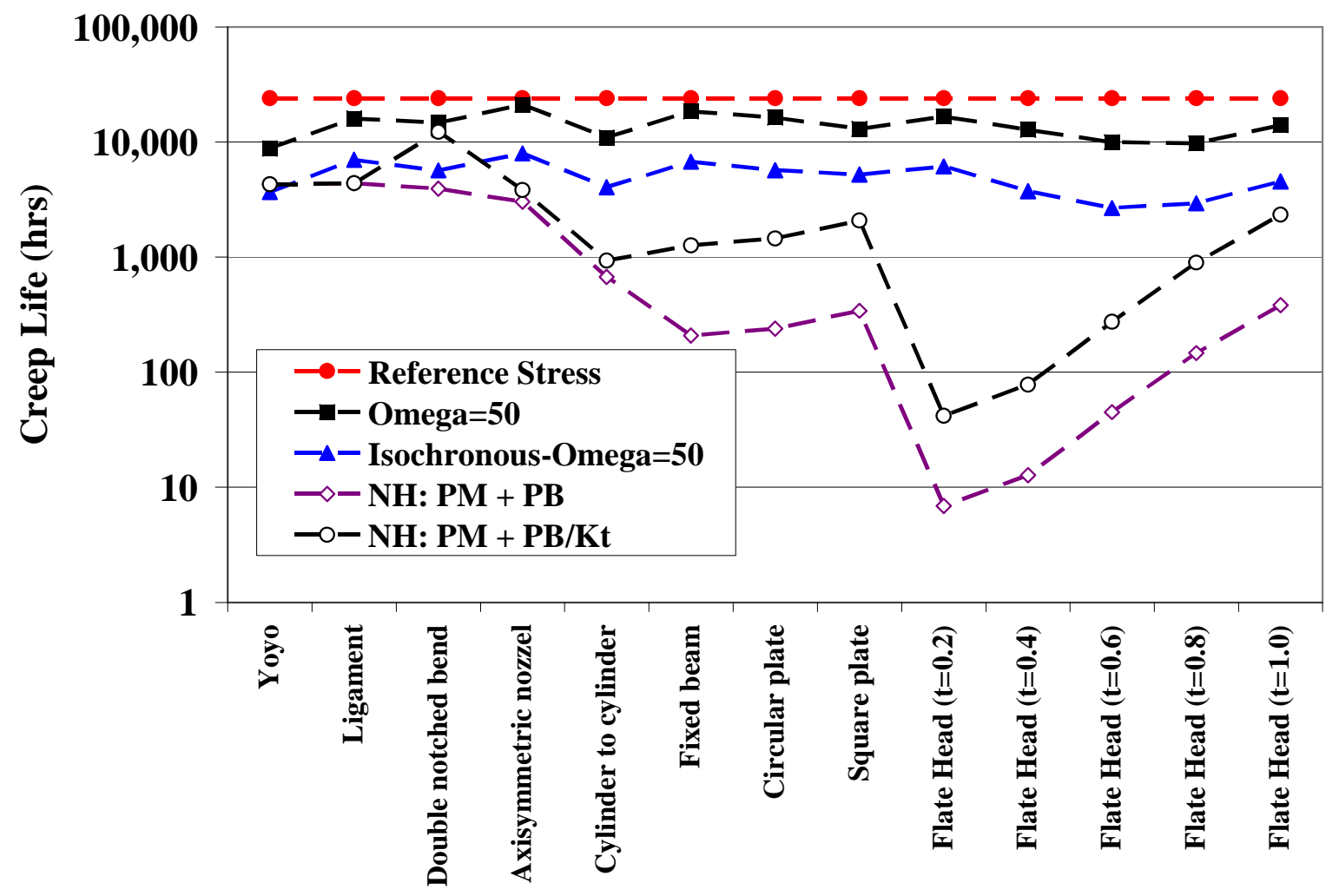

Figure 5 Comparison of predicted creep lives at constant reference stress for notched specimens, pressure vessel components, beams, and plates [19]

Some of the recommendations made with respect to load based design criteria are:

1. The two NH procedures evaluated herein should be retained and unaltered, however, the use of an alternate procedure(s) or the current ASME NH procedures should be permitted. 
2. Alternate design procedures should be considered within $\mathrm{NH}$, namely the use of the limit load reference stress approach, and the use of isochronous curves.

3. Primary stress effects, aging effects, and cyclic softening/hardening effects should be considered in generation of isochronous curves. Different curves can result if based upon constant load, constant strain, or constant strain rate tests or models. These effects may best be predicted with an experimentally verified unified constitutive model. The model might be used to generate a family of isochronous curves to address these issues. Then, the simpler isochronous curves may be used in finite element models as a family of time independent curves to predict time dependent behavior.

In 2006, equivalent ASME NH design criteria were developed based upon the use of reference stress approximations [21]. Equivalent but conservative estimates of the primary stresses in terms of a reference stress were provided. Comparison of the reference stress with stress allowables resulted in proposed changes to ASME NH. This type of approach is specifically noted and permitted in R5. These recommendations have not been adopted in any form in ASME NH to date. These recommendations are consistent with Langer's adoption of the limit load analysis and the basis of its use in NB with a design margin applied to $\left(\mathrm{P}_{\mathrm{m}}+\mathrm{P}_{\mathrm{b}}\right)$ where $\left(\mathrm{P}_{\mathrm{m}}+\mathrm{P}_{\mathrm{b}}\right)$ is arrived at by elastic analysis and stress classification; the difference between the ASME NH (and ASME NB) criteria and the proposed reference stress based criteria are extension of limit analysis to time dependent criteria.

\section{ASME SG-ETD Exposure to the Reference Stress Approach:}

A broad body of work in the area of the reference stress for primary load limits has been conducted and published, much of it utilized in the development of the use of the reference stress in R5; many of these are referenced within R5 [22]. Besides the original implementation of limit analysis by Langer, ASME has been exposed to such information and approaches, including experimental work to verify the reference stress or limit analysis techniques. For example, an excellent report was provided and a presentation 
made by Leckie in October 1983 to the ASME SG-ETD while he was at the University of Illinois in Urbana-Champaign [23]. A copy of this report was included in the August 1985 SG-ETD minutes (Attachment No. 12), and was recently reintroduced to the SGETD in 2006.

Leckie's report includes a variety of test data to support the use of the reference stress for primary load limits and design criteria. Included are test data from aluminum, copper, and 316 SS plates penetrated by holes, aluminum and copper notched round bars, and a variety of weld reinforced pressure vessels made of 21/4Cr1Mo (normalized and tempered) with geometries that included cylinder to cylinder intersections and cylinder to sphere intersections. The reference stress was shown to be very effective in predicting life of structures with notches; the requirement of adequate creep ductility was discussed and demonstrated, consistent with the original work of Goodall [24].

Leckie reported limited success with the reference stress to predict creep rupture of welded structures. For example, in a study conducted by Manjoine of 308 weld metal to join a 304 SS plate, the weld metal has higher creep rupture strength than the base metal. Despite this, cracks first form in the HAZ, and propagate in the base metal at a rate 6 times faster than the weld metal. Manjoine deduces that it appears that crack initiation is governed by the strength of the HAZ, but growth is dependent upon the properties of the base or weld metals. Note, since this time, R5 has devoted lots of attention to weldments and the use of the reference stress.

\section{Reference Stress \& Weldments:}

While the subject is deemed outside of the scope of Task 9, the authors thought it appropriate to make a few comments on the use of reference stress approaches and weldments. Since the reportings of Leckie in 1983, considerable efforts in the use of reference stress (primary load reference stress as well as cyclic reference stress) have been conducted. 
Budden recently published the results of analysis of Type IV creep failure of welded ferritic pressure vessels [25]. Creep rupture and creep crack growth assessments were performed on three $0.5 \mathrm{Cr}-0.5 \mathrm{Mo}-0.25 \mathrm{~V}$ welded pressure vessels using reference stress methods. Each test vessel was observed to fail by steam leakage through the Type IV location of a weldment. Estimated rupture times and failure locations were compared with the experimental results. The location of failure was shown to be correctly predicted and the calculated times to failure to be conservative with respect to the test results in each case. Furthermore, estimates of creep crack growth from a pre-existing defect in the Type IV region of one vessel weldment were also made; conservative predictions of growth close to vessel failure were obtained.

Even more recently, Budden reports on validation of the R5 high-temperature structural integrity assessment procedure for weldements [26]. In this investigation, large-scale component tests were performed to conduct direct substantiation of continued plant operation, validate life monitoring procedures based on accumulated strain or damage, and validate analysis-based structural integrity assessment procedures. Tests on both defect-free and defective components were conducted; Budden discusses the data required from the tests, their use within R5 validation, and their limitations.

Related, Fookes and Smith have proposed a strain based approach to initiation and propagation of defects in weldments [27]. They examine recent developments and methodologies for assessing creep ductile materials, using fracture mechanics parameters like $\mathrm{C}^{*}$ and $\mathrm{Ct}$, that have been extended to include creep-brittle materials. Specifically, they outline the difficulties in adopting these developments. They propose an alternative approach, where a strain based failure assessment diagram (SB-FAD) is used.

Experimental results from a series of tests on a simulated heat affected zone of a low alloy steel were utilized, and the application of the methodology for assessing the initiation and growth of a defect in a creep-brittle material was demonstrated.

Closely related, Carter has reported on efforts to incorporate reference stress approaches and continuum damage approaches to address creep rupture of weldments [28]. In 
summary, he utilized the reference stress approach obtained from limit analysis to analyze a welded structure with different properties for different portions of the weld. The maximum principle stress and Von Mises stresses at integration points in a finite element analyses were easily obtained, and utilized in a continuum damage calculation to predict life. The combination of the reference stress approach with the continuum damage approach was referred to as a 'modified reference stress'.

The approach addressed two major mechanisms: 1) redistribution of stresses due to differences in material properties in the weldment, which tend to protect the weaker material, and 2) multiaxiality effects which tend to increase the ration of maximum principle stresses and Von Mises stresses as redistribution occurs. The approach was illustrated for an Alloy 617 main steam girth weld with different ratios of HAZ width to tube thickness. The R5 approach without multiaxial effects was shown to be nonconservative relative to the 'modified reference stress' approach, while the British standard BS6539 and ASME NH were found to be conservative. The comparisons are seen in Figure 6.

While Carter provides no verification, i.e. test results, the use of continuum damage mechanics is not a new concept and has a long history to merit its use. Details may exist in unpublished DOE reports regarding results of crossweld test data for calibration of the continuum damage model, analysis predictions, and verification testing but were unavailable to the authors. 


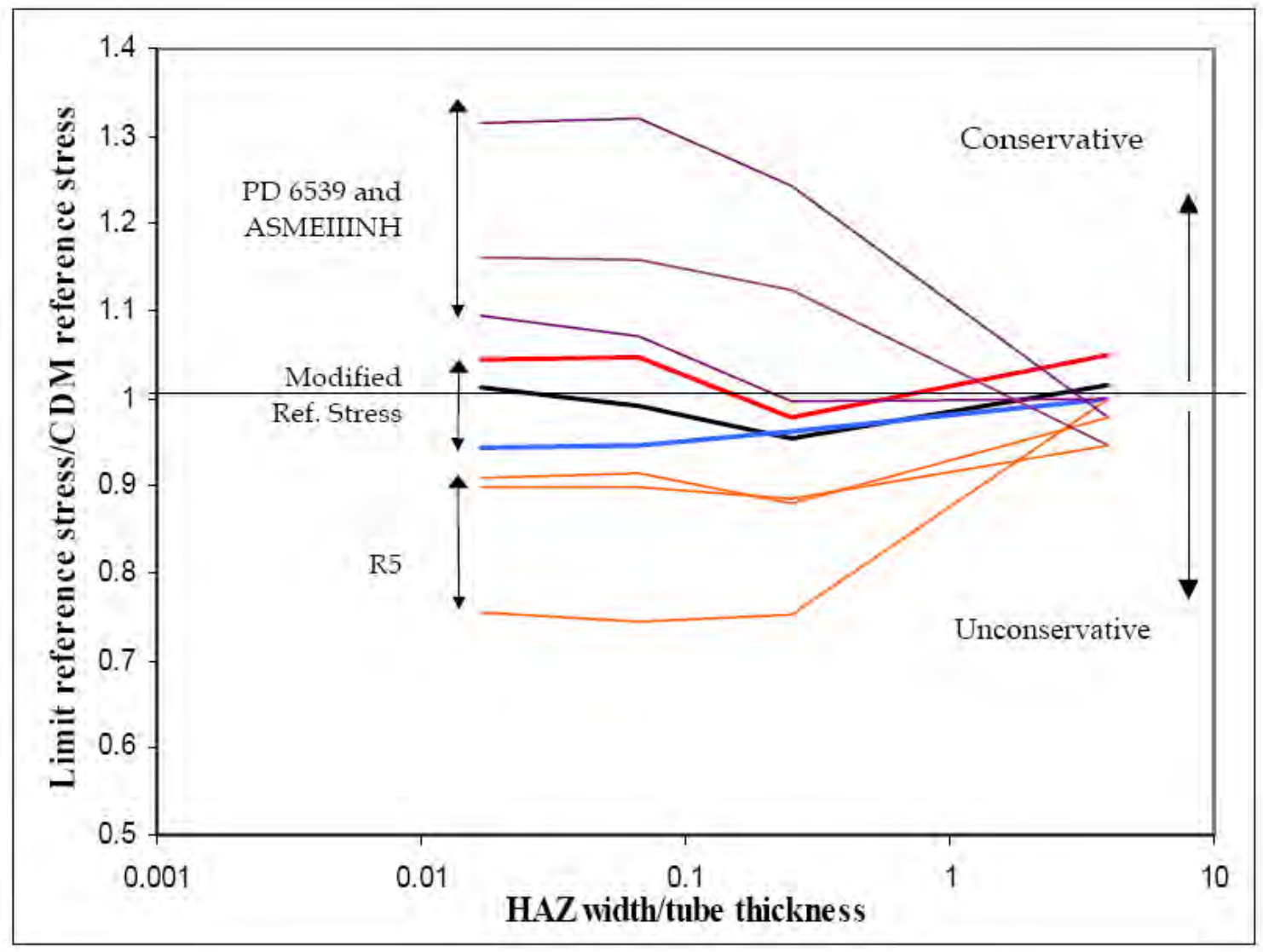

Figure 6 Alloy 617 main steam girth weld [28]

\section{Summary:}

The use of reference stress (limit analysis) to avoid the need of stress classification has been demonstrated and has been used successfully in practice for decades. The use of classical approaches (stress classifications and design by formula) have been shown to be adequate and efficient for some structures, while nonconservative for others; this is apparently, at least partially, due to how stresses may be redistributed and is also dependent upon structure geometry and loading. The requirement of sufficiently creep ductile material is required for use in assessing creep damage (rupture, excessive strain, onset of tertiary creep). Advances in its application to welded structures have taken place, and are used today for the design and construction of structures that operate at elevated temperatures; incorporation of multiaxiality effects and stress redistribution on the evolution of creep damage in weldments is important and realizable. Developments to address creep-brittle materials have taken place with reasonable success. 


\section{PART 4: DEFORMATION CONTROLLED LIMITS}

Application of limit analysis in satisfying Deformation Controlled Limits requires an understanding of the following concepts:

- the steady cyclic state of a structure,

- the definition of a rapid cycle and a slow cycle,

- the role of residual stresses,

- bounds on the rapid cycle solution and the slow cycle solution,

- an elastic core and its associated core stress, core temperature, and core strain,

- the cyclic reference stress (shakedown or ratcheting), and its (their) physical meaning relative to the core stress.

\section{Steady Cyclic State and Rapid vs. Slow Cycle:}

The steady cyclic state of a structure simply means that if cyclic loading were repeatedly applied to a structure, that after some transient response the structure would reach a steady state condition. This steady cyclic condition occurs when the stresses in the structure will vary throughout a cycle with a period $\mathrm{T}$, but would be identical for any two cycles at any given point in time within a cycle, e.g. $\sigma(T+\Delta t)=\sigma(T)$.

Simply speaking, a rapid cycle solution to predicting the steady cyclic state of a structure, or the limit state associated with this steady cyclic state, is one whereby there is insufficient time for creep to take place. In practice, this is accomplished typically by implementing only elastic and plastic material models in the analysis.

Similarly, a slow cycle solution is one where creep is operative, and as such, will influence the steady cyclic state. Since creep is a time dependent mechanism, the rate of loading, cycle time, and dwell are important relative to the rate of creep deformation and the associated creep stress relaxation in obtaining the steady cyclic state. 
The extent of inelastic deformation, whether creep or plasticity, results in redistribution of stresses, where upon removal of loading (mechanical and/or thermal) residual stress fields will be generated. In most cases, these residual stress fields are beneficial; they are beneficial in that they reduce the levels of stress throughout the structure during subsequent cyclic loading relative to the elastically calculated stresses. For example, the redistribution of stresses upon the first half cycle can occur, with subsequent unloading and reloading resulting in elastic loading of the structure. Cases where relaxation of stresses due to creep may not be beneficial include the load case discussed in Task 9.3: where secondary stress relaxation followed by the removal of the applied secondary load, followed by a primary load, or the relaxation of compressive residual stresses that may increase tensile stresses upon reversal of loading during thermal mechanical fatigue loading.

Practically speaking, one way of simplifying the effects of creep on the steady cyclic state is to assume that during a dwell period there is full relaxation of secondary stresses. This requires some restrictions on how long the assumed steady state condition must be assumed to exist relative to subsequent cycles and their steady cyclic state condition. This ensures that the deformation/creep associated with the development of the residual stresses is accounted for in the analysis [3, 29, 30].

Simply speaking, proofs exist and comparison studies have been conducted that demonstrate that use of a rapid cycle provides an upper bound on the stress state of the structure; hence, providing an upper bound (conservative) prediction on the creep deformation/strain of the overall structure, i.e. the core strain or average strain across the section of a structure $[3,4,7,14,30,34,40]$.

When creep is significant, the creep strain predictions based upon rapid cycle solutions may very well be overly conservative. Hence, a more efficient bound on the creep deformation is desired. The most well known efficient creep solution is that for the Bree tube problem, as developed by O'Donnell \& Porowski and utilized in ASME NH 
Appendix T: B-1/B-3 Tests. The approach provides a solution to the steady state slow cycle problem that conservatively bounds creep strain accumulation of the core.

R5 also permits an arbitrary residual stress field to be assumed in order to obtain a more efficient shakedown reference stress, so long as the residual stress field does not change both between cycles and within each cycle, i.e. it remains constant. It is the requirement that the residual stress remain constant that R5 restricts simplified analysis to elastic and shakedown behavior.

Limiting the creep strain representative of the overall deformation of the structure is one of the limits imposed by most ETD Codes, e.g. the average inelastic strain across a section should not exceed $1 \%$ strain.

\section{Core Stress and Shakedown/Ratcheting Reference Stress:}

One method to predict this strain level requires the identification of an elastic core, where the elastic core is the region of the structure that remains elastic during inelastic loading of other regions of the structure; this core provides resistance to deformation to increasing applied loading after other regions of the structure have yielded and have little or no resistance to incremental loading. This core region will experience an associated peak stress level at an extreme end of the cycle (e.g. start-up), and an associated temperature at the extreme end of the cycle (e.g. the temperature at the hot end (start-up) of the cycle). With the assumption that the peak core stress will not relax with time, one can conservatively predict the creep strain accumulation by utilizing the core stress, core temperature, and a simple creep equation or isochronous curves.

Alternatively, one may utilize a different stress to as a representative stress level to bound the inelastic deformation of the structure. In R5, this representative stress is the cyclic reference stress, or more strictly speaking the shakedown reference stress. In order to conservatively bound the strain in the overall structure, one must select a more conservative combination of stress and temperature; the stress and temperature are 
associated with the same location in the structure, e.g. the outer fiber of the tube or the inner fiber of the tube. The shakedown reference stress is defined to accomplish this; that is - the shakedown reference stress is determined by the combination of stress state and temperature that result in the largest creep strain prediction. (Strictly speaking, R5 bases the selection upon the shortest 'rupture' time where 'rupture' refers to the lesser of the stress to rupture and stress to a $1 \%$ or $2 \%$ strain level depending upon the material; since ASME NH Appendix T restricts the strain to $1 \%$, the strain level was used in the definition here for comparison purposes).

Note, the shakedown reference stress is not identical to the core stress; this is due to the fact that depending upon which region of the interaction diagram the structure is operating, different 'mechanisms' can be operating. Let us consider the Bree tube problem, where the elastic core region has been shown to be the mid-plane of the tube wall.

If the loading causes the operating point to be near the ratcheting boundary, e.g. Y 0 and $\mathrm{X}$ approaches $\mathrm{P}_{\text {limit }} / \mathrm{Sy}$ (the limit load of a simple tube, assuming a temperature independent yield strength), the shakedown reference stress will tend to approach or be equal to the core stress. Hence, in this simple case, the shakedown reference stress is associated with the stress at the mid-wall, i.e. the stress at the core region.

For the case where $\mathrm{X} \sim 0$ and $\mathrm{Y}$ approaches 2 (the secondary stress range approaches twice the yield strength, assuming a temperature independent yield), the shakedown reference stress will approach the yield strength - whereas the core stress will be zero; in this simple case, the shakedown reference stress is associated with the stress state at the extreme fibers of the tube, not the mid-wall of the tube. Thus, the shakedown reference stress will conservatively estimate the core stress (overestimate it).

Strictly speaking, R5 defines the shakedown reference stress and temperature as the combination of steady cyclic equivalent stress and associated temperature, $\mathrm{T}$, at the same point during the same period which gives the shortest rupture life. The simple Bree tube 
problem was simply used to illustrate the concept; note, R5 does permit for the Bree tube case that the shakedown reference stress be more efficiently predicted (lower stress) by using the core stress prediction directly.

R5 does not currently permit/utilize a ratcheting reference stress. The ratcheting reference stress concept is similar to the shakedown reference stress, but is synonymous with the core stress of the structure. In the Bree tube problem again (temperature independent yield strength), for cases where $\mathrm{X} \geq 0.5$ and $\mathrm{Y}$ is sufficiently large to cause the operating point to approach the shakedown/ratcheting boundary, the shakedown and ratcheting reference stresses are identical and are both associated with the stress at the mid-wall.

For cases where $\mathrm{X}<0.5$, shakedown occurs at a much lower value of $\mathrm{Y}(\mathrm{Y}=2)$ relative to the intensity of $\mathrm{Y}$ required to cause ratcheting ( $Y \rightarrow \infty$ at $\mathrm{X}=0$ ). In such cases, the ratcheting reference stress will be less than the shakedown reference stress; note, the ratcheting reference stress is associated with the stress at the mid-wall, while the shakedown reference stress is associated with the stress at the fiber extremes. When the operating point approaches the ratcheting boundary for $\mathrm{X}<0.5$ the ratcheting reference stress is identical to the core stress and is associated with the stress at the mid-wall.

Of course, once the cyclic reference stress and reference temperature have been identified, a variety of approaches may be used to predict creep strain - ranging from application of simple Norton-Bailey creep laws, Omega models, isochronous curves, etc for constant stress and temperature conditions. The same is true for approaches that utilize the core stress and core temperature.

In order to implement the concept of a cyclic reference stress (shakedown or ratcheting) as discussed above, the steady cyclic stress state is required. This may be obtained by any one of the limit analysis methods discussed in Part 2. Of course, the method must provide the spatial distribution of stress state and temperature - at least at the extremes of the cycle, i.e. startup and shutdown conditions for the Bree tube problem. (Only the 
stress state is available for shakedown approaches based upon Melan's theorem, and stress and strain state (or strain rate) if the approach is based upon Koiter's theorem.) Note, not all approaches implement temperature dependent properties, e.g. yield strength; in such cases these approaches have limited use in ETD simplified methods.

To illustrate, take an example utilizing a rapid cycle solution or analysis.

Earlier the case of the Bree tube was discussed when $\mathrm{X} \sim 0$ and $\mathrm{Y}$ approaches 2 (Q 2*Sy), with temperature independent yield strength. Clearly, the operating point is at or approaching the limiting shakedown boundary. As such, the shakedown reference stress will be equal to the yield strength of the extreme fiber (inner or outer).

However, what about the case where $\mathrm{X} \sim 0$ and $\mathrm{Y}$ approaches 1.5? To obtain the shakedown reference stress, one common approach is to reduce the yield strength until the shakedown boundary and the operating point are coincident. Hence, in the unmodified yield strength condition, $\mathrm{X}_{1}=\mathrm{Pm} / \mathrm{Sy}_{1} \sim 0$ and $\mathrm{Y}_{1}=\mathrm{Q} / \mathrm{Sy}_{1}=1.5$. Decrease $\mathrm{Sy} 1$ to a value that causes $\mathrm{Y}_{2}=\mathrm{Q} / \mathrm{Sy}_{2}=2$. This occurs when $\mathrm{Sy}_{2}=0.75 * \mathrm{Sy}_{1}$. Hence, the shakedown reference stress for $\mathrm{X} \sim 0$ and $\mathrm{Y}$ approaching 1.5 is $0.75^{*} \mathrm{Sy}$.

Another approach is to simply obtain the steady cyclic stress state spatially and temporally in terms of an equivalent stress state, e.g. Von Mises stress: $\sigma_{M}(x, t)$. Utilizing the associated temperature $\mathrm{T}(\mathrm{x}, \mathrm{t})$, determine the combination that produces the largest creep strain (or shortest rupture time). For the case $\mathrm{X} \sim 0$ and $\mathrm{Y} \sim 2$ (temperature independent yield strength), obviously there is no difference in the shakedown reference stress; it is equal to the yield strength. For the case $\mathrm{X} \sim 0$ and $\mathrm{Y}=1.5$, the outer fibers yield upon first loading, and then shakedown. Hence, at the peak stress state the outer fibers are at a stress level equal to the yield strength. There is no difference in the shakedown reference stress between the two loading cases if this approach is used, as opposed to the shakedown reference stresses obtained by reducing the yield strength until the structure under the given loading conditions just reaches the shakedown limit. 
Let us examine the ratcheting reference stress for the case $\mathrm{X} \sim 0$ and $\mathrm{Y}$ approaching 2 and Y approaching 1.5. Following the same first approach for the shakedown reference stress, reduce the yield strength until the operating point and the ratcheting boundary are coincident. This occurs when the yield strength Sy 0. Hence, the ratcheting reference stress is equal to zero for both cases.

The authors are not aware of a method to obtain the ratcheting reference stress without reducing the yield strength. Reduction of the yield strength can be viewed as using a time and temperature dependent yield strength, where this effective yield strength is equal to the minimum of the temperature dependent yield strength, $\operatorname{Sy}(\mathrm{T})$, and the time and temperature dependent rupture strength, $\operatorname{Sr}(T, t)$ or $\operatorname{St}(T)$. One may simply increase the time, $t$, until the effective yield strength reduces sufficiently to cause the structure to reach the limit state (shakedown or ratcheting) for the given operating cycle of interest. R5 utilizes this procedure to obtain a more efficient shakedown reference stress [22].

By comparison, the elastic core stress is clearly zero for the two operating cases immediately above.

For the simple examples discussed, one can see how conservative the shakedown reference stress may be relative to the core stress. In reality, if the structure were operating at elevated temperature with significant creep relaxation over the duration of lifetime of the structure, the results may be even more conservative relative to the slow cycle solution. A recent investigation illustrated this for the Bree tube problem for Alloy 617 at temperatures above $649^{\circ} \mathrm{C}$; the conclusions also supported that the requirement to utilize unified constitutive models when differentiation between creep and strain rate dependent plasticity was difficult was unnecessary [30].

\section{Types of Cyclic Limit Analysis:}

One may summarize from a broad perspective the use of limit analysis on deformation controlled limits in terms of the following important aspects: 
- rapid cycle vs. slow cycle solutions

- those that identify an elastic core region, core stress, and core temperature, and those that do not,

- those that incorporate temperature dependent yield strength properties, and those that do not, and

- those that incorporate an effective time and temperature dependent yield strength,

Unless otherwise stated, the discussion addresses the intent to limit the average strain in the structure, e.g. the inelastic strain at the mid-wall of the Bree tube problem is limited to $1 \%$ strain in many Codes.

Typically the approaches incorporate elastic perfectly-plastic material models, while some are capable of utilizing various levels of hardening or softening. Structures made of materials that exhibit more hardening than others with the same yield strength will typically be capable of withstanding larger loads than those that are elastic perfectlyplastic. Use of different material models have the following implications: shakedown reference stress predictions tend to increase with materials that harden; ratcheting reference stress and core stress predictions tend to decrease with materials that harden.

Depending upon the combination of approaches used as listed above, either a large body of material exists in the literature to solve such problems or very small. Different degrees of conservatism may result with different approaches. Below is a summary of a combination of such problems and approaches; several known references of solutions/studies from the literature or other resources known. The approaches are grouped further into those that do not identify a core region, core stress and core temperature, and those that do.

Approaches Not Based Upon an Elastic Core:

Approaches that do not identify a core region, core stress and core temperature are: 


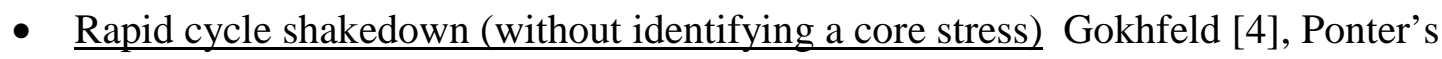
LMM [5, 31, 32], direct cyclic method in Abaqus [33], full inelastic finite element analysis [34], and Vu et. al.'s primal dual method [35] are a few of many.

- $\quad$ Rapid cycle ratchet (without identifying a core stress) Gokhfeld [4], Ponter's LMM [7, 8 ] and full inelastic finite element analysis, e.g. Abaqus [34].

- $\quad$ Slow cycle shakedown and ratchet (without identifying a core stress) Ponter's LMM for arbitrary cycle time [14-16, 36] - which might be viewed by some as closer to a direct route full inelastic FEA method, and full inelastic finite element analysis, e.g. ABAQUS.

One additional complication is consideration of extreme loading conditions in excess of the ratchet boundary. In such case, the plastic ratcheting strain increment is also required. Nearly all simplified approaches fail to address this need. Analytical solutions to specific problems may exist such as a cyclic thermal membrane loading of a thin tube and the Bree tube problem; however, in the context of an approach that is applicable to a wide range of general problems (geometry, boundary conditions, and loading), the only ones that the authors are aware of is the Hybrid method. Incidentally, the Hybrid method is based upon the existence of a core region, not a cyclic reference stress.

With the broad overview of shakedown and ratcheting analysis approaches that do not identify a core region, core stress, and core temperature, a more focused overview of several specific approaches is provided below. The intent is not to provide extensive details of each, but rather to provide the reader with knowledge of the extent of work and type of problems that have been addressed.

\section{LISA:}

The Commission of the European Communities supported a project called LISA (Limit and Shakedown Analysis) for industrial use in the earlier 1990's for about 10 years [17]. In this project, limit and shakedown analysis address the design problem in terms of a nonlinear optimization problem with the objective of determining the maximum safe load. The project specifically did not include approaches such as elastic compensation 
methods, e.g. Ponter's LMM or Seshadri's GLOSS (or R-node) method. This project was a rather large undertaking given the large number of unknowns and constraints for realistic industrial problems considered. The LISA project addressed large scale optimization methods for such purposes. LISA touched on a natural extension of the approach, reliability analysis, which may be of increasing interest to ASME and the NRC.

In summary, some of the methods in the LISA project address temperature dependent properties, and some permit the use of strain hardening materials. All of the approaches can be lumped into the 'rapid cycle' solutions to shakedown analysis. One advantage with these methods is rapid convergence to solutions compared to inelastic direct route approaches. However, these approaches are very sophisticated, as most engineers and pressure vessel designers would unlikely have the time or expertise to understand the theory. At this point in time, the extent that such software is available to designers is limited (at least to the author's knowledge), certainly relative to finite element analysis availability and experience. However, twenty years ago the same may have been said with regards to finite element analysis relative to analytical mechanics solutions with plate and shell theory.

One important aspect for a designer is visual representation to assist in obtaining a physical understanding of the material and structural behavior to loading. In many of the advanced numerical nonlinear optimization approaches, typically no intermediate loading conditions, e.g. stress and strain distribution as loads are ramped up or down, are available to the designer to visualize structural behavior and gain an understanding physically as to the structures response to loading. This is a common drawback for the designer / analyst in gaining an understanding of how structures behave to different loading conditions.

The LISA project included validation of such advanced numerical approaches to the following problems, including primary load limit prediction and/or shakedown limit predictions: 
- a plate with a hole under uniaxial tension,

- a torispherical vessel head under internal pressure,

- a mixing device with high thermal transient, internal pressure, and external piping loads,

- a thin plate subjected to primary and secondary loading,

- a rotating turbine disk with radial temperature distribution,

- the limit pressure of a grooved cylinder,

- the classical Bree tube problem,

- a thick-walled sphere under radial thermal loading and internal pressure, and

- a pipe junction under internal pressure.

While the approaches may be uncommon, they certainly can satisfy the intent of ETD Code to address Primary Limits as well as some of the simplified methods in

Deformation Limits. As such, they shouldn't be eliminated, but maintained as possible options for those well versed in their application. Since these approaches do not identify a core stress, implementation to ETD would require an approach similar or equivalent to that used by R5, a shakedown or ratcheting reference stress approach.

\section{Primal Dual Method:}

Depending upon how the problem is formulated an upper bound or lower bound solution may be obtained. In simple terms, if the problem is formulated according to Melan's static shakedown theory or Koiter's kinematic shakedown theory, lower and upper bound solutions may be obtained to the shakedown limit states being sought. Of particular recent interest is the use of a 'primal dual method' which uses both upper and lower bound formulations in order to converge upon a single, consistent, and true solution [35]. The point is illustrated that some elastic compensation methods are prone to underpredicting limit loads and shakedown limits; this is not a concern in terms of safety as the results will be conservative. 


\section{Gokhfeld:}

Melan and Koiter were pioneers in the area of shakedown, establishing lower and upper bound theorems to such limit states. Gokhfeld, was also a pioneer in the area of limits of structures under cyclic loading, particularly ratcheting limits [4]. He developed many different formulations to the shakedown and ratcheting solutions about the same era of the birth and start of the popularity and use of computers, from about the 1960's to the 1980 's. As such, relative to today, limited advances in implementing numerical methods existed. Some of the approaches in the LISA project and other modern numerical approaches may be utilized in conjunction with Gokhfeld's formulations.

\section{Direct Cyclic Method in ABAQUS:}

The direct cycle method in ABAQUS is an approach that attempts to obtain the stabilized response of an elastic-plastic structure subjected to cyclic loading in a more efficient manner than traditional cyclic analysis (called 'transient analysis' in ABAQUS). As the problem size increases for transient analysis, the solution of these nonlinear equations can dominate the entire computational effort; hence, the method can become quite expensive, since the application of many loading cycles may be required before the stabilized response is obtained. The direct cyclic algorithm uses a modified Newton method in conjunction with a Fourier representation of the solution and the residual vector to obtain the stabilized cyclic response directly.

The basic method can be summarized as follows. A displacement function is defined that describes the response of the structure at all times ' $\mathrm{t}$ ' during a load cycle with period ' $\mathrm{T}$ ' and has the characteristic $\mathrm{u}(\mathrm{t}+\mathrm{T})=\mathrm{u}(\mathrm{t})$. A truncated Fourier series with 'n' Fourier terms is used to represent the displacement function. The problem is reformulated then in terms of an elastic stiffness matrix, correction coefficients to the displacement function, and a residual. The use of the elastic stiffness matrix for the Jacobian throughout the analysis 
results in the requirement to solve the equilibrium equation system only once. Therefore, the direct cyclic algorithm is likely to be less expensive to use than the full Newton approach for the solution of nonlinear equations, especially when the problem is large.

Many similar types of approaches have been developed that do not specifically address creep of structures, two examples being application to train wheels [37], and layered pavements [38]. Spiliopoulos developed a simplified method to predict the steady cyclic stress state of creep structures that utilized Fourier series in the decomposition of residual stresses, rather than displacements as is the approach implemented in ABAQUS [33, 39].

\section{Elastic Compensation Approaches:}

Ponter and colleagues have conducted extensive work in attempts to establish shakedown and ratcheting analysis for structures experiencing creep [14-16]. The approach is an extension of the rapid cycle elastic compensation approach, i.e. Linear Matching Method (LMM). The approach does not identify an elastic core. The LMM approach can be applied to general 3-D structures and general loading.

The LMM is formulated in terms of elastically calculated stresses, residual stresses, and the actual stresses. The formulation and approach relies upon iterative techniques that modify the elastic moduli to perform equivalent inelastic analysis. Loads are prescribed in terms of the constant mechanical and variable mechanical load distributions, and the cyclic thermal load distribution. An elastic load history is calculated. Following a very similar procedure as summarized in Part 2 by Gokhfeld, the elastic stresses are augmented by changes in the residual stresses that simultaneously satisfy equilibrium, compatibility, etc. A load multiplier parameter, $\lambda$, is implemented that operates on the constant mechanical load distribution; the value of $\lambda$ is determined that causes the structure to shakedown or ratchet (for a given variable mechanical plus thermal cyclic load history) - providing a single point on the interaction diagram for the problem in 
question. The process is repeated for various levels of cyclic loading to generate the entire shakedown and ratcheting boundaries.

An alternative formulation does not utilize a load multiplier, but utilizes the reduction in effective yield strength (e.g. $S_{y}=\min \left\{S_{y}(T), S_{t}(t, T)\right\}$ ) as illustrated earlier for obtaining the shakedown stress and ratcheting reference stress for the Bree tube problem. An iterative approach is utilized that increases the time $t$ until the limiting state is reached, i.e. shakedown or ratcheting occurs. The shakedown and ratcheting reference stress are then determined from the combination of the stress and temperature (spatially) that results in the shortest rupture life.

The shakedown analysis approach has been developed for easy implementation in Abaqus via several user subroutines [31]. Several steps are required: determine the vertices of load history in stress space, input of material properties (temperature dependent), thermal analysis (transient if needed) to obtain the temperature field history, performing elastic analysis for the mechanical loads and thermal loads (separately), perform elastic shakedown analysis and demonstrate that reversed plasticity and ratcheting do not occur. If one is concerned with loading in excess of the shakedown limit, an additional analysis is conducted that utilizes the previous shakedown analysis in order to obtain the ratchet limit and/or the ratcheting reference stress.

The LMM procedure also provides information such as the plastic and creep strain range for use in $\mathrm{C}-\mathrm{F}$ assessment; creep strain increments utilize an elastic follow-up calculation.

If the user is interested in obtaining the ratchet limit, the user is cautioned in that the loading path must not exceed the shakedown limit, or the solution will diverge; no such limitation applies to obtaining the shakedown limit.

If a slow cycle solution is sought, the relevant time intervals between vertices of the load history must be prescribed. A modified formulation is implemented which introduces creep deformation to the problem to obtain the residual stresses that are dependent upon 
both plastic and creep deformations. A more accurate solution to the steady cyclic state may be obtained depending upon the number of vertices used to define the load history; as the number of vertices increases (i.e. time between load vertices decreases), the approach essentially becomes nearly identical to an elastic-plastic-creep analysis conducted with specified time steps between vertices.

Much of Ponter et. al.'s work was utilized to verify the recent development of an approach that relies upon the concept of an elastic core - the Hybrid approach [40]. Examples of Ponter's work will be illustrated when the Hybrid approach is summarized.

\section{Approaches Based Upon an Elastic Core:}

Approaches that do identify a core region, core stress and core temperature are:

- $\quad$ Rapid cycle shakedown and ratcheting (with identification of a core stress) The original core stress approach derived from the Bree problem [3], a recent revisited solution to the Bree problem for unequal yield strength cases [41], and a new approach called the Hybrid approach [40].

- Slow cycle shakedown and ratcheting (with identification of a core stress) The same approaches above for rapid shakedown and ratcheting solutions

One quick observation is that there are limited approaches that incorporate the concept of a core region, core stress, and core temperature for cyclic analyses. There may be others, but none for which the authors are aware.

The O'Donnell \& Porowski approach is already utilized in ASME Appendix T (B-1 \& B3 Tests); it is adopted in one form or another by R5, RCC-MR, Monju, and possibly other Codes. As illustrated and restricted with constraints on the core stress in ASME NH Appendix T, the B-1 \& B-3 Tests actually provide solutions to both the rapid and slow cycle solutions for the Bree tube problem. Note, the B-1 \& B-3 Tests do address the case where the yield strength varies on the hot end and cold end of the cycle as well. The 
model is limited in its applicability, e.g. its application to general structures that contain stress concentrations and nonlinear secondary stresses is prohibited.

\section{Bree Tube Problem:}

McGreevy et. al. recently revisited the Bree tube problem [41]. Their findings illustrate that for a material with unequal yield strength at the hot and cold end of the cycle, the shakedown/plasticity boundary for a rapid cycle analysis should correctly be $Q=S_{y L}+S_{y H}$; R5 utilizes a conservative value of $\mathrm{Q}=2 * \mathrm{~S}_{\mathrm{yH}}$. ASME uses a non-conservative boundary of $\mathrm{Q}=2 * \mathrm{~S}_{\mathrm{yL}}$. Note, the delineation of the shakedown/plasticity boundary for the Bree tube problem differentiates between when the extreme fibers shakedown or experience reversible plastic strain; the contours of constant core stress (also called isostrain contours) for the Bree tube are not impacted by the representation of the actual shakedown/plasticity boundary.

The applicability of the B-1 \& B-3 Tests at very high temperatures was investigated by McGreevy \& Abou-Hanna [30]. The study concluded that the use of the B-1 \& B-3 Tests for Alloy 617 in excess of $649^{\circ} \mathrm{C}$ is appropriate, and does not require the use of unified constitutive equations as required in the 617 Draft Code Case. This was confirmed for a) repeated cycles of the identical loading, b) cycles of constant core stress but with varying pressure and linear thermal gradients, c) cycles of variable core stress, and d) simplified block loading. The conclusion also supports applicability of the B-1 and B-3 Test to other materials at temperatures and strain rates where a clear distinction between plasticity and creep behavior is lacking. This includes currently approved ASME Subsection NH materials, with the exception of Mod9Cr1Mo. Mod9Cr1Mo cyclically softens, and appropriate cyclic yield strength properties should be incorporated.

The ASME NH Appendix T simplified design methods, namely the A \& B Tests in T1300, were developed based upon the classical Bree Tube problem. The simplified methods often include restrictions on geometry, temperature, and/or cycle definition to render them suitable for design of most pressure vessels. More advanced approaches 
would be useful in the design of say a compact heat exchanger for the NGNP where the structure and loading are far from a simple tube subjected to constant internal pressure and cyclic thermal gradients.

Appendix $\mathrm{T}$ also places requirements that pressure induced discontinuity stresses and thermal membrane stresses be classified as primary. Pressure induced discontinuity stresses are recognized by the authors to act as primary stresses; however, much controversy exists as to the requirement that thermal membrane stresses be treated as primary for ratcheting analysis - a viewpoint not shared by the authors.

\section{Complex 3-D Structure and General Loading: A Hybrid Approach}

In response to the limitations noted, efforts at ORNL were taken to develop a slow cycle solution (also applicable for rapid cycle predictions) that specifically identifies an elastic core [40] in support of structures operating at very high temperature, such as Very High Temperature Reactors (VHTR), specifically the U.S. Next Generation Nuclear Plant (NGNP). The approach, developed by McGreevy and Abou-Hanna, is a variation of Gokhfeld's fictitious yield strength approach [34, 40]. The simplified method removes restrictions such as linear temperature gradients, geometry, local and gross structural discontinuities, and the need for stress linearization and categorization. This is accomplished while maintaining reasonable accuracy without excessive conservatism.

The Hybrid approach identifies the elastic core region from limit analysis, is capable of conducting primary limit load analysis, cyclic limit state analysis, as well as predicting steady cyclic stress states and ratcheting strain increments [40]. Implementation was achieved with a combination of limit analysis and simplified inelastic finite element analysis for one and a half-cycles. The advantage is a strong physical understanding of a structure's behavior, aided by current computational tools available to the common engineer to arrive at bounded yet not excessively conservative solutions. 
The core regions are identified as those elastic regions in the structure that serve as the overall resistance to deformation of the structure. In terms of limit analysis, the elastic region is the last region of the structure to remain elastic and subsequently yield prior to collapse of the structure under increasing primary loading. The effect of cyclic loads is to reduce the load carrying capacity of the structure, and is a cumulative effect of local reduction in load carrying capacity from local cyclic stresses. This information is readily available from outputs of one stage of the Hybrid approach, the load dependent yield modification (LDYM) approach [34, 40]. An earlier version of LDYM utilized a uniform modification of the yield stress (UMY), meaning the extent by which the cyclic loads reduced the load carrying capacity was independent of the relative direction of the constant primary load and the cyclic loads $[34,40]$. The approach also permits one to identify the type of ratchet mechanism that the structure exhibits as a function of loading, e.g. high primary \& low secondary load combinations may activate one mechanism, while high secondary $\&$ low primary loads activate a different mechanism.

With the core region identified, the core temperature and stress remain to be extracted from the steady cyclic state of the structure. The steady cyclic state of the structure is obtained similar to the process used by O'Donnell \& Porowski [30, 40]. Then, calculation of the creep strain at the core is relatively straightforward.

The Hybrid approach was verified first with benchmark problems in the prediction of ratchet boundaries; various structures and loading conditions were investigated, including thermal membrane stresses, and cyclic primary stresses. Predictions were validated with various alternative approaches, including elastic-plastic finite element analysis, results from the literature, and application of Ponter's Linear Matching Method.

Table 2 summarizes the ratchet boundary problems investigated. Figures 7-9 illustrate the agreement of Ponter's LMM ratchet boundaries with those predicted by the Hybrid. Details of the loading and geometry can be found in [40]; the intent is to illustrate that the simplified approach has been successfully verified for complex loading and structures. Note, the development stage of the Hybrid approach utilized two different approaches in 
the limit analysis step of the procedure; hence, UMY and LDMY refer to these two different approaches. UMY is more conservative than LDMY $[34,40]$.

Table 2 Ratchet boundary problems investigated [40]

\begin{tabular}{|c|c|c|}
\hline $\begin{array}{c}\text { Problem } \\
\text { Description }\end{array}$ & Verification Methods & Comments \\
\hline Bree Tube & $\begin{array}{c}\text { e-p FEA, B-1 Test, Alpha } \\
\text { Model, direct cycle } \\
\text { (ABAQUS), Ponter LMM }\end{array}$ & $\begin{array}{c}\text { rapid' and 'slo w' cycle problems; infinite tube vs. } \\
\text { shorter tube (boundary cond ition effects) }\end{array}$ \\
\hline Bree Plate & $\begin{array}{c}\text { e-p FEA, Ponter LMM, } \\
\text { direct cycle }\end{array}$ & similar to Bree tube, but 2-D (not axisymm.) \\
\hline Travel Wave & $\begin{array}{c}\text { e-p FEA, direct cycle, } \\
\text { Ponter analytical solutions }\end{array}$ & $\begin{array}{c}\text { short' vs. 'long' wave travel distance; st eepness of } \\
\text { thermal membrane gradient }\end{array}$ \\
\hline Holed Plate & Ponter LMM & biaxial pressure loading on ends of plate \\
\hline Holed Plate & e-p FEA, Ponter LMM & axial pressure loading with cy clic thermal gradient \\
\hline Note: e-p: elastic-plastic \\
\hline
\end{tabular}
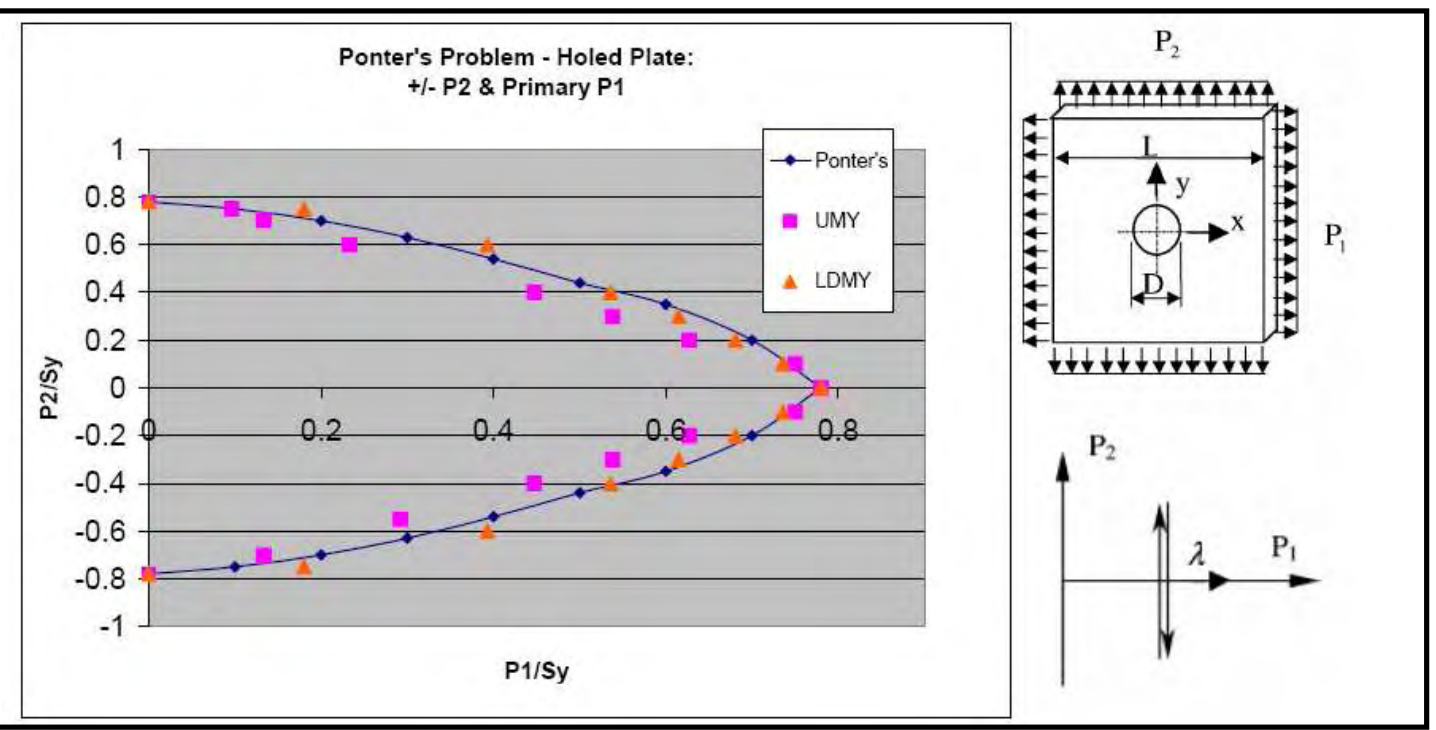

Figure 7 LDYM ratchet boundary verification for the Holed Plate under biaxial loading, zero mean [40] 

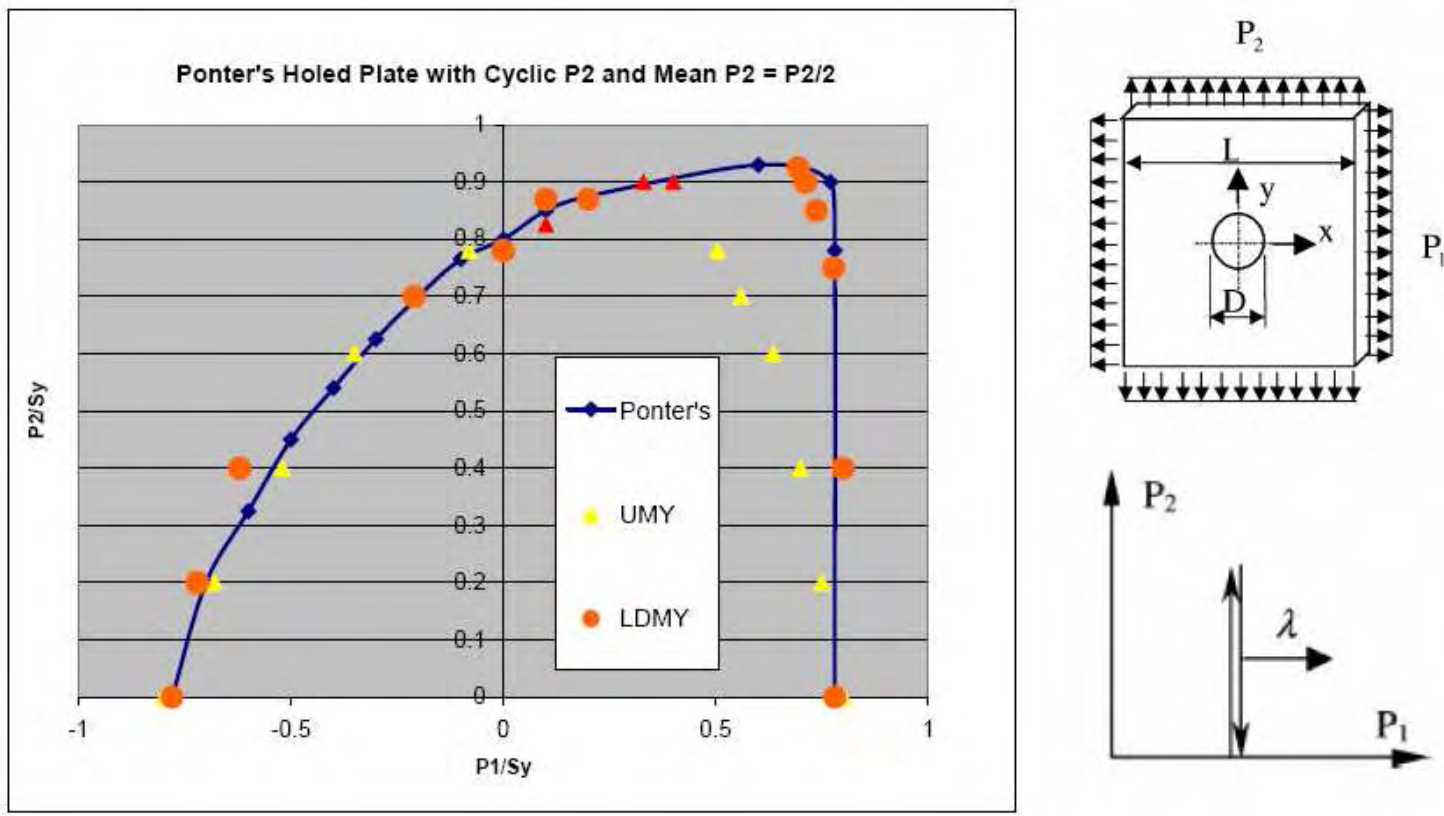

Figure 8 LDYM ratchet boundary verification - Holed Plate: biaxial loading. non-zero mean [40]

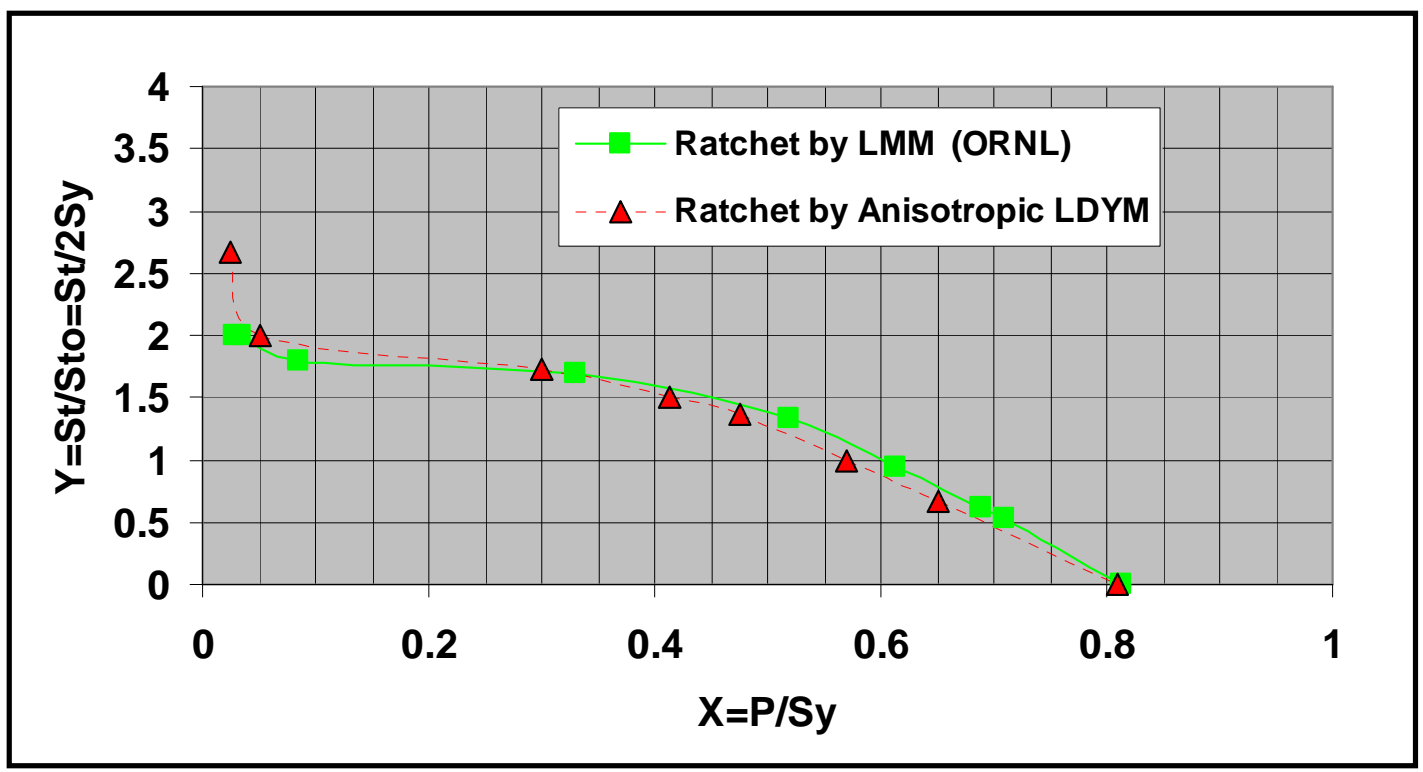

Figure 9 LDYM verification with Holed Plate: constant pressure + nonlinear thermal gradient [40] 
Additional benchmark problems were investigated in the assessment of the accumulation of gross structural deformation or strains, namely the Bree Tube problem and Ponter's Holed Plate problem. The Holed Plate Problem is a case where a nonlinear cyclic thermal gradient is applied to a plate with a hole, which is also subjected to constant and/or cyclic primary loads; the problem may appear somewhat simple at first glance. However, the problem introduces significant peak strains due to geometry (large notch/hole) and nonlinear thermal loading. As such, the problem poses challenges with application of current ASME NH Appendix T simplified methods, e.g. limitations of geometry, loading, cycle definitions, and temperature restrictions. Various loading conditions and material properties were investigated for the Holed Plate problem as well, utilizing Alloy 617 material properties. For instance, a case was developed where the temperature cycle was intentionally defined so that the elastic core region would be at a relative cool or low temperature during the dwell period in order that the extent of creep deformation would be very low. In another example, the temperature cycle was intentionally defined so that the elastic core region would be at a very high temperature during the dwell period so that very significant amounts of creep took place. Results were verified with full inelastic cyclic creep finite element analysis.

The Hybrid approach may be implemented in either rapid or slow cycle solutions; this permits analysis of conditions where relaxation of secondary stresses is either limited or non existent (rapid cycle) or significant (slow cycle). Consequently, the effects of carryover or residual stresses associated with the relaxation on the elastic core stress may be addressed. In such cases, the degree of conservatism associated with rapid cycle solutions is drastically reduced when a slow cycle solution is implemented. The rapid cycle was observed to predict as much as 30-60 times more creep strain than the slow cycle solution. This is consistent with the findings of a similar comparison of rapid vs. slow cycle solutions for the Bree tube problem [30].

Relative to full inelastic finite element analysis, the slow cycle Hybrid approach predicted creep strain accumulation of the core that 2-10 times higher than the finite element analysis. Grouping of cycles into 'blocks' was also investigated; the block 
representation with slow cycle solution resulted in substantially large conservatism in strain prediction, on the order of 50 times more than FEA results; this is consistent with the finding in [30].

Strain linearization of the inelastic analysis results was also conducted. A comparison was made with direct observations of the strains in the structure, as well as the predicted core region of the structure. The conclusions made were that strain linearization per ASME Appendix T was inappropriate in some circumstances, and conservative in others; the results support the concept of an elastic core to predict gross structural behavior rather than strain linearization.

Prediction of plastic ratchet strains was also shown to be possible with the Hybrid approach, illustrating that the Hybrid solution is applicable to elastic, shakedown, plastic, and ratcheting regimes. This is important, as many simplified solutions are limited to elastic (A Tests), and/or shakedown and plasticity regimes (B-1 \& B-2 Tests). The approach may be applied on a cycle by cycle basis, or block of cycles, similar to the B1/B-3 Tests in ASME NH Appendix T. Note, R5 limits use of simplified methods to the shakedown regime, with restrictions that the assumed residual stress field state be utilized and demonstrated to be constant with respect to time throughout all loading cycles for the assessment of shakedown.

While not part of the scope of the investigation, more efficient core strain predictions might be achieved by integration of the Hybrid approach with an elastic follow-up factor, but the results need to be demonstrated to be conservative and may require restrictions on how/when the such an approach may be used.

The Hybrid approach may be readily extended as a simplified inelastic C-F analysis method, since the analysis readily provides stress and strain (elastic and plastic) information throughout the load history of the cycle or block in question; for example, the use of elastic follow-up may provide conservative estimates of the creep strain range increment. Depending upon the type of damage rule to be implemented (time fraction or 
ductility exhaustion), the elastic follow-up permits creep damage calculations to be made. One may conservatively assume an infinite elastic follow-up, e.g. no relaxation of stress during a dwell. Or, one may use a previously demonstrated conservative elastic followup factor for the structure and cycle of interest, e.g. rather than conduct creep relaxation analysis for each cycle, a study that demonstrates a range of various follow-ups may conservatively bound the problem for later use in simplified methods. Finally, one may conduct a monotonic creep relaxation calculation to obtain the creep strain increment directly.

Note, the extent of relaxation will be a function of the structure, the loading, and will be limited to relaxation to a stress level equal to the primary reference stress or the core stress; a conservative estimate would be to limit the relaxation to the core stress. As such, the Hybrid approach should be directly applicable to providing inputs for assessment of $\mathrm{C}-\mathrm{F}$ in the structure.

In terms of computational effort, the CPU and 'clock' time was not documented; however, the Hybrid approach was easily 10 times faster, typically 100-1,000 times faster than full inelastic finite element analysis. In addition, a strong understanding of the structure's behavior is gained. The net result is a better understanding of the problem in a fraction of the time.

The extent of detail placed into the finite element model, either in terms of geometry and/or load history, may vary depending upon how far along one is within the design stage: e.g. conceptual to final design, and even fitness for service. Furthermore, since the approach can be used for elastic, shakedown, reversed plasticity, or ratcheting states, the approach lends itself to implementation in various design criteria, e.g. equivalents of the ASME NH Appendix T A-Tests \& B-Tests, or structures with much more complex 3-D geometry and loading. In addition, the extension to $\mathrm{C}-\mathrm{F}$ analysis is clearly very feasible.

\section{Summary:}


The difference between a core stress and a reference stress (shakedown and ratcheting) has been illustrated through several simple examples. The ratcheting reference stress has been shown to be equivalent to the core stress, while the shakedown reference stress has been shown to be not necessarily associated with the core stress and to be more conservative than the core stress. A review of various types of cyclic limit analysis methods was provided, emphasizing those that are and are not based upon the concept of an elastic core. Those that are not based upon an elastic core region lend themselves to implementation similar to a cyclic reference stress.

A summary of two promising methods, the Linear Matching Method (LMM) and the Hybrid approach were provided. LMM is not based upon an elastic core concept, while the Hybrid is. Both are able to predict the ratcheting boundary; most methods are incapable of doing so. Rapid and slow cycle solutions are feasible with both LMM and the Hybrid; slow cycle solutions have been shown to be very efficient under conditions where creep is significant, while rapid cycle approaches have been shown to tend to be overly conservative. Both methods can be extended for use in C-F assessment. Complex 3-D structures subjected to complex loading (e.g. nonlinear thermal gradients) may be analyzed by the LMM and Hybrid approaches. The two approaches lend themselves to application at various stages in the design and/or operation process. Finally, relative to full inelastic analysis, both the LMM and Hybrid approach are faster, the Hybrid being 10-1,000 times faster.

\section{SUMMARY}

Elastic analysis, reference stress analysis, and limit load, shakedown, and ratcheting analysis are all variations of the use of 'limit analysis'. Elastic analysis provides a lower bound on the limiting state of a structure, while inelastic analysis provides a means of a more efficient and accurate estimate of the limiting state of a structure. Note, various inelastic approaches exist that provide lower or upper bounds on inelastic solutions. 
Limit analysis is actually already utilized extensively by ASME, as well as all Codes that are based primarily upon ASME. However, limit analysis is largely implemented in ASME by use of elastic analysis, rather than inelastic analysis; whereas some Codes implement inelastic analysis to obtain more accurate predictions of the limit state, e.g. R5's use of primary reference stress.

For primary load limits, two illustrative studies of analysis methods to satisfy primary load limits were reviewed: the first with respect to time independent limits, and the second with respect to time dependent limits. Realistic structures and loading comprised the various examples investigated. Application to primary limits of weldments was also noted.

For deformation controlled limits (e.g. cyclic limits), simple examples were utilized to illustrate how modern approaches can be used in terms of the reference stress approach and the concept of an elastic core and core stress. References were cited that illustrate various points, such as the use of slow cycle solutions as more efficient bounds on creep strain accumulation then rapid cycle solutions.

Two promising simplified methods were discussed briefly as well: Ponter's Linear Matching Method (LMM) and McGreevy and Abou-Hanna's Hybrid approach. Ponter's method is based upon reference stress concepts, while McGreevy and Abou-Hanna's Hybrid approach is based upon the concept of a core region and core stress. Both approaches lend themselves naturally for extension in C-F analysis. The two approaches can be utilized in elastic, shakedown, plastic, and ratcheting analysis as well; most of the alternative simplified methods are limited to assessing shakedown and are incapable of addressing behavior in the plasticity and ratcheting regimes. Results were reviewed relative to full inelastic analysis for the case of a plate with a large hole in it subjected to cyclic nonlinear thermal gradients and constant primary loading.

The use of these modern simplified methods provides for more efficient predictions of relevant stress states and can be readily integrated with existing design criteria. Solutions 
can be obtained very quickly, on the order of 10-1000 times faster than full inelastic analysis. The physical behavior of the structure can be more readily understood with the simplified results, as compared to full inelastic analysis - particularly when utilizing the concept of a core stress. Such methods also lend themselves to contain as much detail or lack of detail as necessary, lending themselves to be applied to design criteria with decreasing degrees of conservatism for use in pre-conceptual to final design stages, e.g. from use of the A-1 Test (conservative), to the B-3 Test (less conservative). 


\section{REFERENCES}

1. Criteria of the ASME Boiler and Pressure Vessel Code for Design by Analysis in Sections III and VIII, Division 2, A Decade of Progress. B.J.Langer Commemorative Volume, Volume 1 - Analysis for Design, pp.61-83.

2. J. Bree, Elastic-Plastic Behavior of Thin Tubes Subjected to Internal Pressure and Intermittent High-Heat Fluxes with Applications to Fast Nuclear-Reactor Fuel Elements, Journal of Strain Analysis, Vol. 2, No. 3, 1967.

3. O'Donnell and J.S. Porowski, Upper Bounds for Accumulated Strains Due to Creep Ratcheting, Trans. ASME, Journal of Pressure Vessel Technology, Vol. 96, pp. 126, 1974; also WRC Bulletin No. 185 (1974).

4. D. A. Gokhfeld and O.F. Cherniavsky, "Limit analysis of structures at thermal cycling", SIJTHOFF \& NOORDHOFF 1980.

5. A. R. S. Ponter and M. Englehardt, "Shakedown limits for a general yield condition: implementation and application for a Von Mises yield condition," Eur. J. Mech. A/Solids 19, 423 (2000).

6. Seshadri, R., Fernando, C.P.D., 1992, "Limit Loads of Mechanical Components and Structures using the GLOSS R-Node Method," Journal of Pressure Vessel Technology, Vol. 114, pp. 201-208.

7. A.R.S. Ponter and H. Chen, A minimum theorem for cyclic load in excess of shakedown, with application to the evaluation of a ratchet limit, Eur. J. Mech. A/Solids 20 (2001) pp 539-553.

8. A.R.S. Ponter and H. Chen, A method for the evaluation of a ratchet limit and the amplitude of plastic strain for bodies subjected to cyclic loading, Eur. J. Mech. A/Solids 20 (2001) pp 555-571. 
9. D.L. Marriott, Evaluation of Deformation or Load Control of Stresses under Inelastic Conditions using Elastic FEA, ASME Publ. PVP - Vol. 136, ASME, 1988.

10. Seshadri, R., "The Generalized Local Stress Strain (GLOSS) Analysis - Theory and Applications," Journal of Pressure Vessel Technology, Vol. 113, pp. 219-227, 1991.

11. D. Mackenzie, J.T. Boyle, and R. Hamilton, The elastic compensation method for limit and shakedown analysis: a review, Journal of Strain Analysis, Vol. 35, No 3, pp. 171-188, 2000.

12. Seshadri, R., Marriott, D.L., 1993, “On Relating the Reference Stress, Limit Load and the ASME Stress Classification Concepts," Int. J. Pres. Ves. \& Piping, Vol. 56, pp. 387-408.

13. H. Hagashima et. al., Application of a Classification Method to Obtain Primary Stresses Without Evaluation Sections to Perforated Structures, Proceedings of PVP2005, PVP2005-71690, July 17-21 Denver, Colorado, USA, 2005.

14. M. Boulbibane and A.R.S. Ponter, Minimum theorems and the Linear Matching method for bodies in a cyclic state of creep, European Journal of Mechanics A/Solids, 21, pp-915-925, 2002.

15. M. Boulbibane and A.R.S. Ponter, A method for the evaluation of design limits for structural materials in a cyclic state of creep, European Journal of Mechanics A/Solids, 21, pp. 899-914, 2002.

16. M. Boulbibane and A.R.S. Ponter, Finite element analysis and evaluation of design limits for structural materials in a cyclic state of creep, International Journal for Numerical Methods in Engineering, 58, pp. 2065-2082, 2003.

17. "Numerical Methods for Limit and Shakedown Analysis: Deterministic and Probabilistic Problems. Report of the European project: FEM-Based Limit and Shakedown Analysis for Design and Integrity Assessment in European Industry LISA," Publication Series of the John von Neumann Institute for Computing (NIC) Volume 15, (2002). 
18. Design by Analysis, 17 December 1999, http://www/mpa-lifetech.de/EPERCTP/ ped/jrc/ jrc_design.html (no longer available online), Pressure Equipment Directive: Joint Research Centre; contact: European Pressure Equipment Research Council, http://www.eperc.bam.de/projects/ttf1.html

19. T. E. McGreevy, D. L. Marriott and P. Carter, "High-Temperature Design Methods Development Advances for 617: Status \& Plans," ORNL/TM-2005/515, Oak Ridge National Laboratory (2005).

20. Design for Creep, 2nd Edition, Penny \& Marriott, Chapman \& Hall 1995.

21. T.E. McGreevy, Interim Development of Simplified Methods for Very High Temperature Metallic Design, ORNL/GEN4/LTR-06-16, Oak Ridge National Laboratory, Oak Ridge, TN, July 2006.

22. R5, Assessment Procedure for the High Temperature Response of Structures, Issue 3, British Energy Generation Ltd, June 2003.

23. F.A. Leckie, Damage Studies in Pressure Vessel Components, UIUC, Urbana, IL, 1983.

24. Goodall, I.W., Cockroft, R.D., and Chubb, E.J., "An Approximate Description of the Creep Rupture of Structures", Int. J. Mech. Sci, Vol. 17, pp.351, 1975.

25. P.J. Budden, Analysis of the Type IV creep failures of three welded ferritic pressure vessels, International Journal of Pressure Vessels and Piping, Volume 75, Issue 6, pp. 509-519, May 1998.

26. P.J. Budden, Validation of the high-temperature structural integrity procedure R5 by component testing, International Journal of Pressure Vessels and Piping, Volume 80, Issues 7-8, pp. 517-526, July-August 2003.

27. A. J. Fookes and D. J. Smith, Using a strain based failure assessment diagram for creep-brittle materials, International Journal of Pressure Vessels and Piping, Volume 78, Issues 11-12, pp. 951-961, November 2001. 
28. P. Carter, Simplified Methods for High Temperature Weld Design and Assessment for Steady and Cyclic Loading, EPRI report, 2007.

29. ASME NH Appendix T, 2007.

30. T.E. McGreevy and J. Abou-Hanna, Applicability of Simplified Methods to Alloy 617 in Excess of $649^{\circ} \mathrm{C}$, PVP2007-26672, ASME PVP Conference, San Antonio, TX, July 22-26, 2007.

31. A. R. S. Ponter and H. F. Chen, "Linear Matching Method on Structural Integrity Assessment - User Guide," Department of Engineering, University of Leicester \& British Energy Generation Ltd. (2004).

32. H.F Chen and A.R.S. Ponter, Integrity assessment of a 3D tube-plate using the linear matching method. Part1. Shakedown, reverse plasticity and ratcheting, International Journal of Pressure Vessels and Piping, 82 pp 85-94 (2005).

33. ABAQUS vs. 6.5.1.

34. T.E. McGreevy, C.E. Duty, and R.L. Williamson, Status of the Development of Simplified Methods and Constitutive Equations, ORNL/TM-2006/544, Oak Ridge National Laboratories, Oak Ridge, TN, August 2006.

35. D.K. Vu, M. Staat, and I.T. Tran, Analysis of pressure equipment by application of the primal-dual theory of shakedown, Communications in Numerical Methods in Engineering, Vol. 23, No 3, pp. 213-225, 2007.

36. H. F. Chen and A. R. S. Ponter, "Shakedown and limit analyses for 3-D structures using the linear matching method," International Journal of Pressure Vessels \& Piping, Vol. 78, No 6, 443-51, 2001.

37. M. Bocciarelli, G. Cocchetti and G. Maier, Shakedown analysis of train wheels by Fourier series and nonlinear programming, Engineering Structures, Volume 26, Issue 4, March 2004, Pages 455-470. 
38. I. F. Collins, A. P. Wang, L. R. Saunders, Shakedown in layered pavements under moving surface loads, International Journal for Numerical and Analytical Methods in Geomechanics, Vol. 17, No 3, pp 165-174, 1993.

39. K.V. Spiliopoulos, A Simplified Method to Predict the Steady Cyclic Stress State of Creeping Structures, Transactions of the ASME, Vol. 69, pp. 148-153, March 2002.

40. T. E. McGreevy, and J. Abou-Hanna, A Hybrid Approach to Simplified Methods, ORNL subcontract report: Contract \#6400007059, October 27, 2008; to be published.

41. T. E. McGreevy et al., "The Effect of Temperature Dependent Strength on Upper Bounds for Creep Ratcheting”, PVP2006-ICPVT11-94011, July 23-27, 2006, Vancouver, BC, Canada, 2006.

42. L.C.Hsu, Introduction to Part I - Analysis for Design, A Decade of Progress. B.J.Langer Commemorative Volume, Volume 1 - Analysis for Design, pp.1-2.

43. R.L.Cloud, Introduction to Chapter 1 - Design Criteria, A Decade of Progress. B.J.Langer Commemorative Volume, Volume 1 - Analysis for Design, pp.61-83.

44. M.D.Bernstein, Design Criteria for Boilers and Pressure Vessels in the USA, $6^{\text {th }}$ Int. Conf. on PVT, Beijing, PRC, Sept 11-15 1988, PVP-Vol. 152, pp. 111-138.

45. Design Analysis, Robust Methods and Stress Classification, ASME PVP Conf., Denver, Co., July 25-29 1993.

46. D.C.Drucker, W.Prager and H.J.Greenberg, "Extended Limit Design Theorems for continuous Media", Quart. Appl. Math., Vo. 9, 1952, pp. 381-389. 


\title{
Task 9: Update and Improve Subsection NH - Simplified Elastic and Inelastic Design Analysis Methods
}

\section{Subtask 9.5 - Recommendations for Revisions of ASME ETD Code Gen IV / NGNP Materials Project}

\author{
Submitted to \\ Mr. Jim Ramirez \\ Vice President, Business Development \\ ASME Standards Technology, LLC \\ Three Park Avenue \\ New York, NY 10016 USA \\ By \\ Advanced Consulting Engineering Services, Inc. \\ Peoria, IL \\ On behalf of \\ Jeries Abou-Hanna \\ Douglas L. Marriott \\ Timothy E. McGreevy
}




\section{TABLE OF CONTENTS}

SUBTASK OBJECTIVE....................................................

9.5.1. REVIEW OF EXISTING ETD ASPECTS OF THE ASME CODE...........4

9.5.2. RECOMMENDATIONS .............................................

9.5.2.1 General Comments on the Needs for Change.............................6

9.5.2.2 Summary of Recommendations..................................... 14 


\section{SUBTASK OBJECTIVE}

\section{“Everything should be made as simple as possible, but not simpler” Albert Einstein}

The objective of this subtask is to offer recommendations for modifications to ETD methods presented by the ASME BPV Code, resulting from the work carried out in Tasks 9.1 through 9.4 of this project.

A reminder is appropriate at this point, that the scope of this project is limited to simplified methods with application to primary load design and conformance with ratcheting analysis to satisfy deformation limits due to monotonic and cyclic loading. In particular it concentrates on the following aspects of analysis.

- Elastic analysis,

- Reference stress methods,

- Limit load, shakedown, and ratcheting analysis.

Note, these three areas are a subset of the Ideal ETD Code and all International ETD Codes discussed and reported in Subtasks 9.1 and 9.2. These topics are addressed in terms of Primary Load Limits (elastic analysis, reference stress methods, and limit load analysis) and Deformation Controlled Limits (elastic analysis, shakedown and ratcheting analysis, and the use of limit load and/or reference stress methods) to address elastic/shakedown/plasticity/ratcheting analysis. 


\subsubsection{REVIEW OF EXISTING ETD ASPECTS OF THE ASME CODE}

In order to establish a baseline from which to recommend change, it is worthwhile briefly reviewing the current status of the ASME BPV Code with respect to ETD.

"Elevated temperature" is, for the most part synonymous with "in the creep range". Three main Sections of the Code address this operating regime, Section I, Section VIII/Division 1, and Section III, Division 1, Subsection NH. The first two of these Sections are limited to steady load conditions in terms of the specific rules and guidelines they provide. Although they both call for consideration of all anticipated operating conditions, including cyclic loading, the designer is left to deal with such eventualities in ways he, with agreement with the owner, decides to be most suitable. Only Subsection NH attempts to deal with the problem of elevated temperature operation in any detail.

The design criterion used in Sections I and VIII/1 is a single allowable limiting stress based on material properties, which must not be exceeded by component stresses. Time dependent elevated temperature properties, i.e. creep properties, are accounted for in this criterion by limiting the stress to cause creep rupture or $1 \%$ strain in a nominal period of 100,000 hours. Thereafter, once the design allowable has been set based on temperature, no further consideration is given to time dependence as part of the component design process. Calculation of the component design stress is invariably mandated in these Sections of the Code for certain well defined geometries, by the use of handbook-type "strength-of-materials" methods, or using tables or graphs derived from more detailed studies using fundamental elasticity theory or Finite element analysis (FEA). Interestingly, Sections I and VIII/1 do not necessarily prohibit the use of alternative methods of computation, as long as the method can be agreed on with the Owner, and is accompanied by a plausible validation, i.e. "mandatory" procedures do not necessarily prohibit alternatives.

Subsection NH evolved from a series of Code Cases written to provide guidance in the design of the FFTF sodium cooled fast reactor. Due to the large, cyclic thermal transients anticipated in this system, and the safety related concerns of the nuclear industry, the simple methods offered in Sections I and VIII/1 were deemed inadequate and work began to develop design rules for dealing with cyclic loading, in particular thermal transients in the creep range. Unlike Sections I and VIII/1, NH recognizes the time dependence of ETD and provides design allowables which are a function of service life.

The end result of this evolutionary process is a Code section (NH) with the following attributes.

1. It is the only place in the Code to deal explicitly with the time dependent nature of component operation in the creep regime.

2. It is the only place in the Code where complex operating conditions in the creep range, such as cyclic loading are addressed explicitly.

3. It is built around temperature regimes and component geometries most likely to be encountered in FFTF operation, both of which focus the problem area so as to 
make the task of developing simplified methods of analysis easier than might be the case for a more general class of problem. The downside of this focus is uncertainty with the possibility of expanding the application to the more general problem class.

4. Understanding of the creep phenomenon itself, as well as the mechanics of components operating in the creep range were both virtually in their infancies as far as design was concerned, at the time the Code Cases which grew into $\mathrm{NH}$ were first proposed. While progress has been made in both of these fields, inevitably, working developments into a Code is a time consuming task, as witnessed by the fact that the immediate predecessor of $\mathrm{NH}$, Nuclear Code Case N47, went through more than 40 revisions over 2 decades.

While undoubtedly a pioneering effort as far as ASME Code development is concerned, it can also be assumed that, with the additional demands likely to be made by the operating requirements of candidate reactors being considered as part of the Generation IV program, NH will need some modifications and updates.

Some modifications that have been recognized, and are being explored in other tasks of this ASME ST LLC research program, include expanding the list of accepted materials from the current five to include more high temperature alloys and increasing the upper temperature limits. For example, Section I allows design to go beyond the current range of applicability of the stress tables in Section II, Part D, if an alternative basis for setting design allowables can be validated. $\mathrm{NH}$ prohibits such freedom of action, limiting the designer strictly to the temperature limits set in Section II. To advance to higher temperature applications therefore, some work needs to be done to establish increased temperature limits as well as completely new materials.

Again, it is emphasized that the focus of this Task 9, and of this report on Subtask 9.5, is limited in scope to what, if anything, can be done in the way of methods for simplified analysis to expand the applicability of $\mathrm{NH}$. Materials, their ranges of applicability and the question of design criteria are not items for discussion in themselves, although issues involving these topics surface at times. In such cases the procedure in this report will be to stop short at asking a question and will not proceed beyond that point to offering recommendations.

In working up to this point, several preliminary steps have been taken.

Subtask 9.1 attempted, without reference to any specific code, standard or guideline currently in existence, to map out what it is believed an "ideal" ETD Code should encompass.

Subtask 9.2. reviewed a number of existing, internationally recognized codes (to the extent that it was possible to obtain information on other codes and standards), independently and without comparison to ASME NH. The review encompassed guidelines for scope, and for the use of methods of analysis, with particular emphasis on the use of simplified methods. This task was carried out in the belief that some of the 
best lessons about what should be included in an ETD code could be learned by examining what had been done elsewhere, i.e. benchmarking.

Subtask 9.3 attempted to identify areas where NH could benefit by following or drawing from the practices advocated in other codes and guidelines, i.e. conducting a direct comparison of the findings in Subtask 9.2.

Subtask 9.4 reviewed a group of more recent analysis methods which have appeared in some form in the technical literature but have yet to be fully absorbed into established design code practice.

Subtask 9.5 is a viewpoint, based on these four preceding subtasks, on changes that are believed to offer some benefit to continued development of $\mathrm{NH}$.

\subsubsection{RECOMMENDATIONS}

\subsubsection{General Comments on the Needs for Change}

1. One widely recognized feature of future ETD is increased, possibly very radically increased, operating temperatures. Material properties have long been known to depend on temperature and, wherever possible, attempts have been made to accommodate such variations into design calculations. This variation has the general appearance of a relatively slow change with increasing temperature in the regime where time independent properties govern design allowables, changing to a sharp cliff-like drop-off at the point where time dependent (creep) properties begin to become very significant. Up till now, most pressure vessel applications have hovered around, or slightly below the "cliff top" (Figure 1), where temperature dependencies are significant but contained in a range of 10 to 50\% or so of some nominal value. The much higher temperatures envisioned for the future push the application point down to the foot of the "cliff", where even moderate temperature differences can represent very large property variations, which may be higher than current approximate methods can cope. 


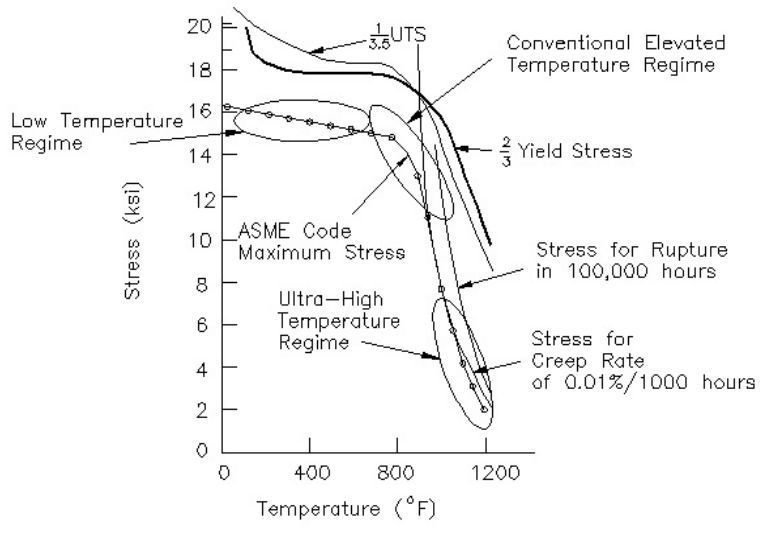

Schematic Diagram of ASME Design Stress Determination

Figure 1 Effect of temperature on material properties.

2. Computational technology has changed in the time $\mathrm{NH}$ and its precedents have evolved. Where simple approximations were necessary less than 10 years ago, today, with the exponential increases in affordable computing power, it is literally easier in many cases to carry out a detailed analysis than to attempt an approximation - assuming the necessary information on geometric details and material properties exists to fuel the detailed analysis. And in this single sentence one of the changes in the motivation for simplified or approximate methods has been captured. The question is not "how to make the problem simple enough to do with limited resources?", but "how to make sensible use of almost unlimited computing resources to solve problems for which there is limited, or unreliable input data". One of the answers to this question is to search for so-called "robust" methods, of which the "Reference Stress" technique is one, which are capable of providing reliable solutions, preferably safe bounds, but not too conservative, using the minimum of input data. For instance, calculating a Reference Stress solution is no more or less computationally difficult than doing a transient creep analysis using a Bailey/Norton n-power law. The difference is that the Reference Stress solution does not depend on "n", which is not known for many materials listed in Section II, Part D, and can be proved, rigorously, to give an accurate upper bound to the deformations predicted by the full creep analysis. In summary, the issue is not how easy it is to do the calculation, but how accurate a prediction one can make with the sparse, to nonexistent at times, material data available as input to the analysis.

3. One approximation in particular deserves singling out for special scrutiny. This is the stress categorization procedure summarized in Table NH-3217-1, together with the associated, mandated, use of linear elastic analysis as the basis for stress analysis in evaluation of primary loads. This is a well established and widely used technique which has been validated by decades of successful application; but, it has known limitations when attempts are made to apply it to the complex threedimensional geometries being considered as a routine matter in current design 
work. It is also inherently conservative, which is no bad thing in design but, as temperatures increase and the useful range of material properties narrows as a result, there is a strong incentive to examine all conservative assumptions and eliminate or reduce any that might be excessively so. It is recommended that the NH Code encourage the use of finite element technology and provide guidelines for interpreting FEA results. Further, it is recommended that the Code provide finite element examples in a non-mandatory appendix that support the FEA guidelines. The Appendix could include reference to key pertinent publications.

4. As an addendum to (2) above, the methods used by engineer/analysts have also changed radically in the past two decades or so. "Hand calculations" considered routine a short while ago are, regrettably, falling into disuse in favor of computer based solutions for even the most trivial problem. This genie will not go back into the bottle, and it is believed that one of the inevitable changes that needs to be made in Code formulation is to recognize the new direction being taken in computational engineering and present its rules and procedures accordingly. This is not to be taken as a suggestion to eliminate 'hand calculations', but to offer additional routes based upon use of advanced computational tools used in engineering practice today.

5. Evaluation of cyclic deformation and conformance with strain limits is a section of $\mathrm{NH}$ where there is opportunity for improvement. While it is recognized in the body of NH-3000 that this is a complex nonlinear problem, guidance on how to deal with it is limited. Ultimately, the designer, in consultation with the Owner, is free to use whatever methods are deemed fit to define load cycles, evaluate the behavior of complex components under these load cycles, and to define criteria of acceptability. However, specific guidance offered in the form of a nonmandatory Appendix T, written around a simplified nonlinear piping model that had special significance for evaluation of the cylinder-like components envisioned for the FFTF, has room for improvement in terms of general use for ETD. Although the scope of this approach has been extended as NCC N47, the precursor to NH, evolved with time, the scope of this methodology is restricted when compared with the complexity of the cyclic problems encountered today and, conceivably, in future design activities. The approximate methodology set out in Appendix T is possibly adequate for dealing with problems that fall within its range of applicability. As with primary load evaluation, good reasons still exist for simplified methods to deal with this complicated problem area. The issue is that, due to advancing computational capabilities and increased sophistication in the definition of component operating conditions, the range of applicability of the Appendix $\mathrm{T}$ methodology is now possibly too narrow to encompass all applications of practical interest, and the task of expanding its range to cope with the new situation is arguably more difficult than simply making a new start and taking advantage of modern developments such as the exponential improvements in nonlinear FEA.

As for the body of $\mathrm{NH}$, the Subsection is, for the purpose of this discussion, comprised of three main elements. These are, 
1. The Foreword and Article-3000, which together set out the responsibilities of the designer, the design philosophy of the Subsection, the criteria to be observed, and such computational procedures designated as mandatory. This element also specifies in detail the compliance with criteria governing primary load carrying capability.

2. Mandatory Appendix I, which lists the materials and their properties to be used in Code related calculations.

3. Nonmandatory Appendix $T$, which provides optional criteria and approximate methods for assessing deformation limits and material damage under cyclic loading conditions.

Element 1 contains the procedures necessary to assure integrity against gross structural collapse. In common with the low temperature NB, NH uses the classification of stresses into "primary", being the stresses required to resist structural failure under externally applied mechanical loads, "secondary" for self-equilibrating residual or discontinuity stresses, and "peak" for local stress concentrations. The method mandated by $\mathrm{NH}$ at present for calculating stresses and for factorizing the total stress distribution into "primary" and "secondary” is linear elastic analysis.

Furthermore, the design criteria are written in a form which ties them rigidly to the stress categories defined by the simplified elastic analysis procedure.

Despite the fact that elevated temperature operation is possibly the one regime where structural behavior is emphatically not linear elastic, the decision to take this route was made, apparently, because of the uncertainty which existed among ASME Code developers, in the earlier years of NH's evolution, on how to deal with the nonlinear problem. The chosen solution was conservative and served its purpose, but the nonlinear nature of operation in the creep range is well understood today and no longer poses any analytical problem. Therefore this is an area where modification and updating can be beneficial.

There are alternatives for calculating primary load resistance, notably the Reference Stress technique, which has an established pedigree and has been adopted, or is in the process of being adopted, by several other international codes and guidelines. Unfortunately, the output of this method is not immediately compatible with the format that has been adopted in NH for design criteria. For instance, subdivisions of stress into categories, such as "primary membrane" and "primary bending" have no equivalents in the Reference Stress procedure. Therefore, whatever the merits enjoyed by the Reference Stress as a future modification to $\mathrm{NH}$, harmonizing the procedure with the design criteria, by modifying one or the other, or both, is an unavoidable step.

The problem is not insoluble. In fact the advantages that lie in primary load analysis using limit analysis has been long recognized in the low temperature portions of the ASME Code, such as Section III, subsection NB, and its non-nuclear companion, 
Section VIII/Division 2 and there is a recognized procedure for doing so (see NB-3228.1 Limit Analysis).

NB solves the problem of stress classification, for the purpose of primary load analysis, by dispensing with the classification process completely, replacing it with a simple safety factor of 1.5 on limit load collapse assuming a fictitious "yield stress" equal to $S_{\mathrm{m}}$, which amounts to calculating the "collapse load" assuming the yield stress to be the design allowable.

It is tempting to consider adopting a similar procedure for elevated temperature design, by replacing $\mathrm{S}_{\mathrm{m}}$, the low temperature design allowable with $\mathrm{S}_{\mathrm{m}}$, the elevated temperature allowable. In fact this would be exactly how the Reference Stress concept would be implemented if it were not for the need to conform to the specific requirements of the NH design criteria.

The first hurdle to be overcome is the wording of $\mathrm{NH}$ which, for Design and Levels A,B and C Service Limits mandates linear elastic analysis. Only Level D Service Limit admits the option of inelastic analysis. Even an optional or nonmandatory procedure based on the Reference Stress, or any other nonlinear analysis, is prohibited by this wording. It is recommended that the Code allow such other options for Levels A, B and C Service Loads.

Even if nonlinear procedures are permitted by a wording change, problems still exist in the structure of the design criteria themselves.

Design limits are not a problem, since the criteria, (equations (1) and (2) of Para. NH-3221.1) are essentially the same as those used for Design limits in NB, (Fig NB3221-1).

The problem first arises in Level A and B Service Limits. In both NB and NH, the short term collapse criteria for Design are based on general structural collapse, on the assumption that plastic deformation will be fully developed, at least on individual sections, with yielding across the entire section to form a mechanism which, in bending would be a plastic hinge. In NH the criterion, equations (4), is nearly identical to NB (identical if $\mathrm{K}=1.5$ ). $\mathrm{NH}$ equation (4) was modified to produce equation (5) by attempting to allow for redistribution of stresses from the elastic state to the steady creep state. Hence, the intent of equation (5) was to approximate the maximum stress (at a point) on the section. In other words, Equation (5) was modified by $\mathrm{K}$ to account for creep redistribution of stresses to predict the maximum stress under steady state conditions. Equation (4) has an effective safety factor of 1 for pure bending of a beam, and 1.5 for pure axial loading. Equation (5) effectively predicts a stress that is at least $25 \%$ higher than the Reference Stress, e.g. a safety factor of 1.2.

The implication is that $\mathrm{NH}$ assumes failure by creep to occur on a section when the extreme fiber reaches a critical damage equivalent to a tensile test at the maximum steady state stress. This assumption means that local stress relaxation from high local stresses ("F" stresses) is being ignored in the process of evaluating creep life, but the 
redistribution accompanying creep "damage" propagation due to the additional relaxation caused by the onset of tertiary creep, as modeled for instance using the Omega creep model (see API 579), is not.

An added complication is the fact that the "creep" related component of the design criteria is a combination of several possibly unrelated physical phenomena, each of which translates differently from a statically determinate tensile test to a highly stressed element of material embedded in a large statically indeterminate structure.

In the first place, $S_{t}$ is the minimum of two criteria based on two completely different physical phenomena. These are, firstly, creep "rupture”, or failure of a tensile test under a nominally constant stress in a certain time and, secondly, an accumulated creep strain in a similar time.

Further to this, failure of a tensile specimen by "rupture" can be caused by several quite distinct mechanisms. The common wisdom is that "rupture" is the result of void initiation and growth, which is observed at the macroscopic level as "tertiary" creep. Tertiary creep, since it is presumed to be a sign of void formation, is perceived to be a "bad thing". On the contrary, tertiary creep, whether caused by voids or any other mechanism, is a desirable phenomenon from the point of view of a redundant structure, because it allows the stress at the highest stressed location to relax, so reducing the damage rate locally, and prolonging the life of the component, sometimes by orders of magnitude.

This is not the appropriate place to try and solve this problem, but merely to point out the fact that, before simplified methods such as the Reference Stress technique can be reconciled with the current structure of $\mathrm{NH}$, some additional thought needs to be put into how the results of the Reference Stress calculation can be made compatible with the form of the design criteria listed in NH. No doubt, the answer will be reached by deliberations in the ETD SWG.

The main issues involved in Element 2, Material properties, are related to selection of additional materials and extending temperature ranges and are strictly outside the scope of this report. However, some input from analysis is appropriate due to the changing needs for material data needed to drive any computational modifications, i.e. to properly implement choices of creep failure criteria for definition of stress allowables with design criteria that utilize a calculated stress to compare against a stress allowable.

Within the scope of current reporting, it would be of great benefit in the application of some candidate approximate methods to have the deformation and rupture criteria presented separately, instead of the combined format used at present. Measures of the minimum creep rate (mcr) and time-to-rupture provide sufficient information to construct a plausible working model of creep behavior including the tertiary phase which is now believed to predominate in conditions of very high temperature in most instances. Further, it would help to provide ductility data, i.e. \% elongation and \% reduction of area. Between these and a simplified model of tertiary creep deformation, and a generic multiaxial ductility criterion, such as the Rice-Tracey equation, it is possible, with very 
little additional input or computational effort, to assess component failure due to both distributed and localized creep damage. It should be noted that this recommendation does not call for the collection of any additional material data, but merely to report what is already measured in a more fundamental format.

Beyond the material properties already mentioned, a measurement not commonly made on failed creep specimens, but one which holds useful information for component failure assessment, is the diametral strain, or reduction in area, at failure, at locations remote from the failure site, or neck if necking occurs. This dimension may not be available for tests carried in the past, but it should be included in the post-test specimen dimension check in future tests. The reason is that it provides an immediate estimate of the so-called "Monkman-Grant" strain which, together with the common conventional measures of ductility permits an estimate to be made of the " $\lambda$ " factor, a quantity referred to in the British R5 document and used as a criterion to judge whether creep rupture is likely to occur in a distributed and therefore gradual fashion, or in a localized fashion which would lead to creep cracking.

To assist in the more complex analyses associated with cyclic loading Appendix $\mathrm{T}$ already recognizes the need for creep deformation data and provides this information in the form of isochronous stress-strain curves. Currently these curves are provided in graphical form. For computational purposes it would be far preferable to provide the raw algebraic models from which the curves were developed.

Additional material data which is not currently provided in $\mathrm{NH}$ but would be invaluable for the purposes of cyclic loading is cyclic stress-strain data and stress relaxation curves. There is a precedent in the ASME Code for providing this data. Annex 3D - Strength Parameter of Section VIII/Division 2, 2007, lists monotonic and cyclic stress-strain data as a function of temperature for a range of alloys (Tables 3D.1 and 3D.2). At minimum this information should be provided for any alloys accepted for use in nuclear applications. The value of such has also been noted in earlier DOE-ASME GenIV Materials Tasks on creep-fatigue, where the use of cyclic stress-strain curves, provides a more appropriate prediction of stress, and hence creep damage, for materials that cyclically soften, e.g. Gr91.

The satisfaction of deformation limits is possibly the most difficult aspect of ETD. This difficulty is reflected in the fact that specific guidelines in $\mathrm{NH}$ are confined to nonmandatory Appendix $\mathrm{T}$ and then to a limited range of cyclic loadings and component geometries ${ }^{1}$. This area continues to be a difficult one to grasp at the design stage, when knowledge of the final system is still in a state of flux. Here some need for approximate methods of analysis is unavoidable. The methods contained in Appendix $\mathrm{T}$ appear to be satisfactory, as far as they go, since they have been in use and under continuing development, in some form or other, for more than three decades. The only truly plausible method available at present to evaluate cyclic loading is believed to be detailed

\footnotetext{
${ }^{1}$ Note, $\mathrm{NH}$ permits other approaches in the Design Specification - assuming ample justification is made for such approaches; given the fact that the NRC has not approved or disapproved ASME NH and that no elevated temperature nuclear reactors have been licensed by the NRC nor supported based upon ASME NH rules to set a precedent, reactor vendors may be reluctant to take any alternative routes.
} 
nonlinear finite element analysis. From discussions in companion literature which seeks to help in interpreting the Code, opinion seems to be leaning toward this viewpoint among some Code developers (ASME Companion Guide, Chapter 12, para. 12.3.9.6). In fact, the ASME Companion Guide references WRC Bulletin 363 May 1991, which includes among other simplified methods the Reference Stress approach for Primary Stress Limits and the Shakedown Reference Stress approach for Shakedown Analysis and associated limits. It is certainly true in the down-to-earth environment of design and engineering consulting, anything less than a fully detailed nonlinear FEA based assessment, is likely to see rejection by the client/owner unless compelling evidence can be put forward to justify any simplified or approximate approach that might be offered as an alternative. Taking the detailed option can mean greater cost and expenditure of time but, in the present computational climate, where computing capability is easily available and comparatively cheap, simplified methods are a hard sell unless they have been rigorously tested against realistic baseline problems. This does not negate the value or future use of simplified procedures (on the contrary, consultants rely upon them often as in-house guides on which detailed analysis may be planned, in order to reduce efforts and costs), but it signifies that they need to possess very good pedigrees.

In summary, a lack of computing power is no longer an excuse for carrying out a simplified analysis, of the "hand calculation" variety in place of a detailed finite element based analysis. The primary potential value in simplified methods of analysis now lies in the ability to provide robust conclusions, that is to say conclusions that continue to be valid when made on the basis of preliminary, incomplete or uncertain data, as is invariably the case in design. The use of the reference stress for Primary Load limits is a great example. Shakedown and ratcheting analysis - be it reference stress or core stress based approaches, are the equivalent approaches for cyclic or Deformation Limits. If any doubts remain, one way forward is to accept the use of detailed analysis as the de facto design tool of choice, but provide a forum where candidate simplified methods can be compared against a baseline of detailed solutions. At such time as one of these candidates reaches a satisfactory standard of performance, it may be considered then to be incorporated into the Code as either a recommended nonmandatory procedure or a mandated one.

The platform for a test site such as this already exists within the Code, in the form of the nonmandatory Appendix structure. This structure incidentally also provides a solution to the problem of how to bring useful supplementary information to the attention of the Code user, because it is possible, within a nonmandatory Appendix, to provide a list of references. In Section II, for instance, there are three nonmandatory Appendices, L, $\mathrm{N}$ and $\mathrm{W}$ which include extensive bibliographies. ASME will need to determine whether to continue this practice or not.

Given that this is 2009, and the Internet is a household tool, it would seem most appropriate to place the forum for comparison and testing of candidate approximate methods on a website. The mechanism to implement such a move is already in place within the ASME organization, in the form of C\&S Connect. 
The aim of this website forum is most emphatically not to formulate design rules or criteria. This is the job of the Code committee and its organs. In the age of FEA the spectrum of component geometries is proliferating and may reach a level where it may no longer be feasible to encapsulate all design rules as standard solutions to standard prototype geometries, Particularly when considering complex service conditions the nature of a simplification is likely to take the form of a short cut to the characterization of a loading cycle, for instance, where the geometry may be a highly detailed model, but the difficulty in the analysis lies in the time and cost of completing a stable, convergent solution. Proposals for improved efficiency, accuracy or sheer ability to converge to a stable solution may only be capable of validation by direct application to realistic problems. The aim of the proposed website would be to offer a platform where candidate methods can be posted in sufficient detail to be downloaded, tested independently, and reviewed by anyone with an interest in and the capability of doing so. This would form, in effect, an ongoing, adaptive round robin the results to date being a source of potential additions to Code practice as and when methods have developed the requisite maturity and are perceived by the Code body to meet a recognized need.

It is to be noted that this proposal has a precedent; Reference [Wolfgang Hoffelner, PVP 2009-77692], already contains a proposal for just such a site for preliminary on-job testing of material property data. A companion site to deal similarly with the problem of analytical procedures is therefore not a very radical proposition.

\subsubsection{Summary of Recommendations}

1. The main body of $\mathrm{NH}$ should be left as is, with one change to NH-3000, which is to revise the wording attached to design procedures for Design, Service Limits A, B and C to permit alternative, nonmandatory procedures for the purpose of primary load evaluation. Optional methods, such as the Reference Stress method should be documented in an additional nonmandatory Appendix TH. This Appendix can contain reference material following the practice laid down in nonmandatory Appendices L, N and W of Section III. In the area of primary load evaluation, the Reference Stress method is believed to have developed a sufficient body of supporting data, including its adoption, or proposed adoption, in other international ETD codes and guidelines that it deserves serious consideration for incorporation into the code as an optional procedure. There is already a precedent within Section III (NB-3228.1 Limit Analysis) permitting a method for low temperature evaluation which is all but in name the Reference Stress procedure. With an adjustment to accommodate concerns about local creep, for which R5 has a proposed solution, this method can be implemented with little or no modification to $\mathrm{NH}$ as it stands today, or at a minimum as an alternative procedure in non-mandatory Appendix TH.

2. Strictly from the viewpoint of supporting design calculations, material data reported in Appendix I needs to be modified to incorporate the material 
parameters listed in paragraph 9.5.2.1 above, including but not necessarily restricted to separate listing of creep and rupture criteria, ductility measures, monotonic and cyclic stress-strain curves.

3. Nonmandatory Appendix $\mathrm{T}$ should be left at a high level largely untouched, i.e. maintain use of A and B Tests. The limits of applicability of the methods in Appendix $\mathrm{T}$ need to be tested more rigorously and restated to reflect the current environment in which work of this nature is carried out. Specifically, revisit:

a The lack of geometry / loading restrictions and cyclic temperature restrictions (due to possible very high temperature applications) on the A-Tests should be revisited as discussed in SubTask 9.4.

b The applicability of the B-2 Test to general structures; the loading in this study consisted of very simplistic thermal gradients and simple, largely axisymmetric geometries which pale by comparison with the kinds of structures and load histories considered routine by modern day standards. A simple example of a typical thermal cycling problem is included as Attachment A of this report for comparison.

c Modifications to clarify word selection for the B-3 Test as discussed in SubTask 9.4 report are recommended.

4. It is recommended that the Code include non-mandatory appendices (Appendix TH and TT) to provide guidance and examples for alternative methods and procedures for Load Limits and Deformation Limits, respectively; the contents may serve as a non-mandatory appendix to multiple sections of the Code, e.g. Sec III Div 5, Sec III SubSection NH, and Code Case N-201. It is recommended that the modern simplified methods, discussed in report of subtask 9.4, be considered to be included as alternative design analysis methods after a suitable period for independent peer evaluation posted on the website proposed in 5 below. Of course, an appropriate ASME committee with a charter to approve or disapprove any recommendations for additions to Appendix TH and/or TT would be required.

5. Develop a website on which candidate analytical methods can be posted, available to anyone with an interest in testing them, with a mechanism for recording user feedback on utility, errors, examples of possible applications, etc. The priming package could be the examples to be collected to form the basis of a round robin, being the defined scope of Subtask 9.6 of this project. In time, the range of problems could be expanded to follow current interests and priorities. At any time, the ongoing status of the work recorded on this website could be drawn upon for acceptable methods for inclusion in the proposed nonmandatory Appendices TH and Appendix TT, described above.

6. Optional methods of evaluation of cyclic loading should be described in nonmandatory Appendix TT, accompanied by references to background 
documents and examples illustrating the application of the method in sufficient detail to be useable as templates for design projects

7. Procedures and guidelines for strain linearization in inelastic analysis should be described in nonmandatory Appendix TT, with examples illustrating the linearization procedure.

8. Stress linearization method should be described in nonmandatory Appendix TT, along with examples illustrating the linearization approach. 


$$
\begin{gathered}
\text { Subtask } 9.5 \text { - Attachment A } \\
\text { Mixing Tee }
\end{gathered}
$$

\section{Example of thermal/Mechanical load cycle in creep range}




\section{Mixing Tee - Comments on example}

This example does not purport to present the solution to the cyclic problem.

Its objective is limited to demonstrating the complexity of geometry and temperature gradients to be expected in even a relatively simple thermo-mechanical cycle.

Cyclic behavior is demonstrated by direct computation of plastic strains and total (plastic + creep) deformations for 2 successive cycles.

Note that even with 3-d geometry simplified to axisymmetry, thermal gradients remain complex 
Model of 3-D Tee

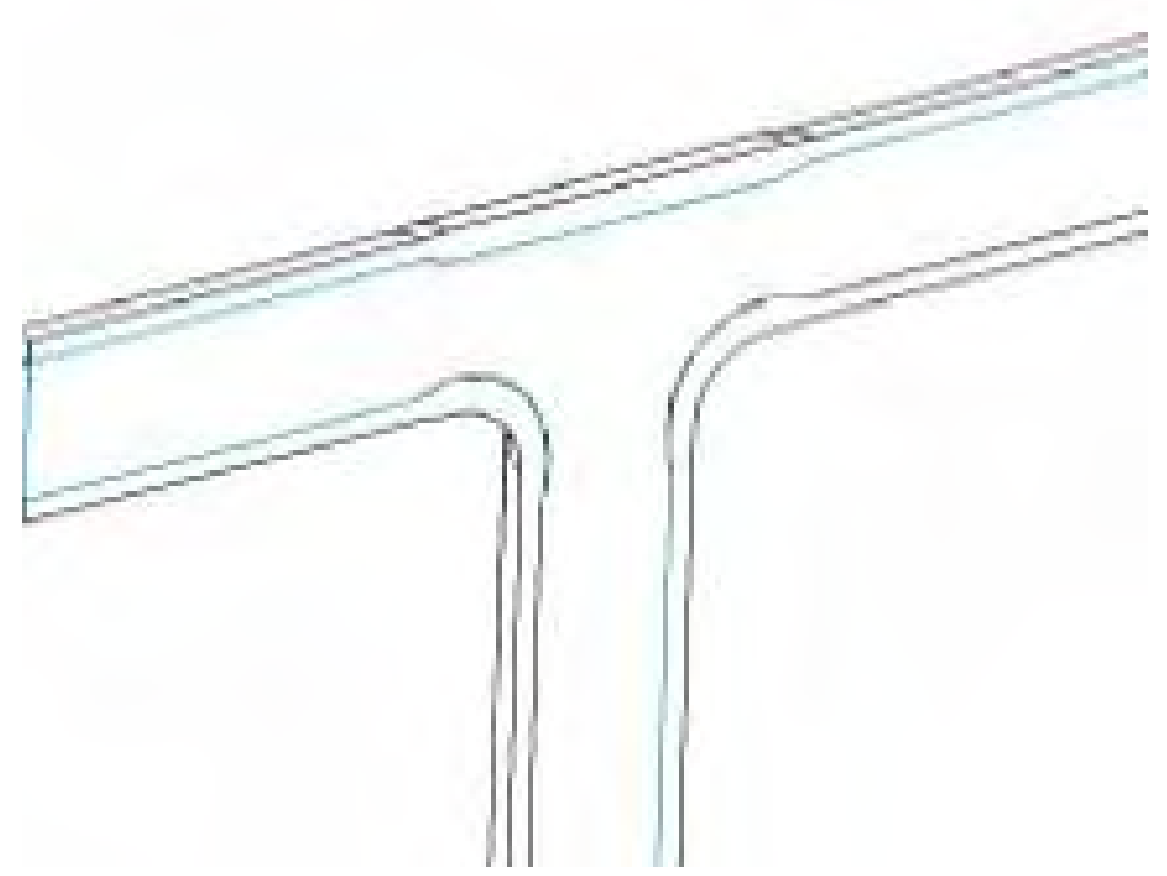

3 
Typical temperature profile on heatup

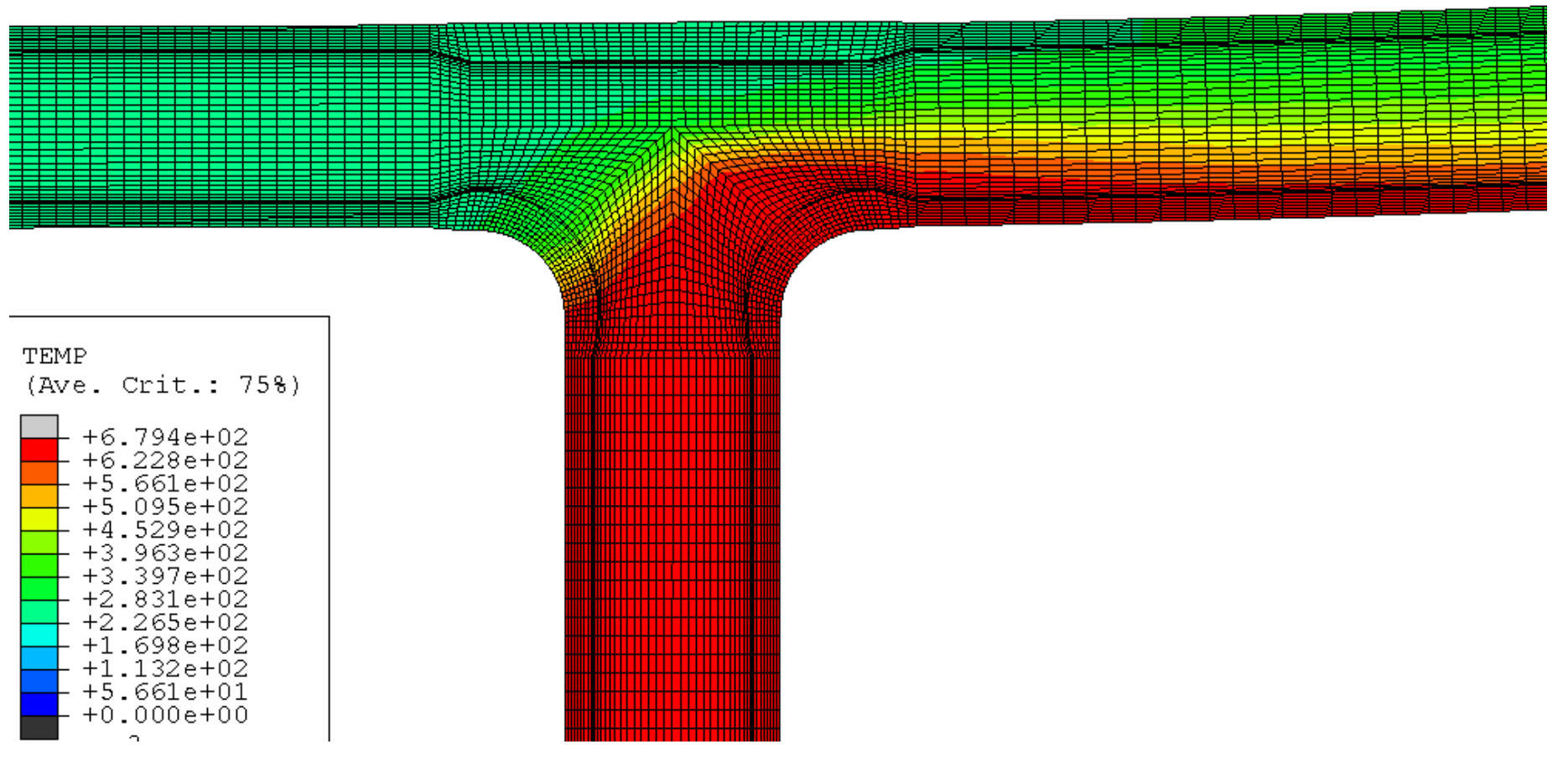




\section{Fatigue curves for 304SS from Section III/NH}

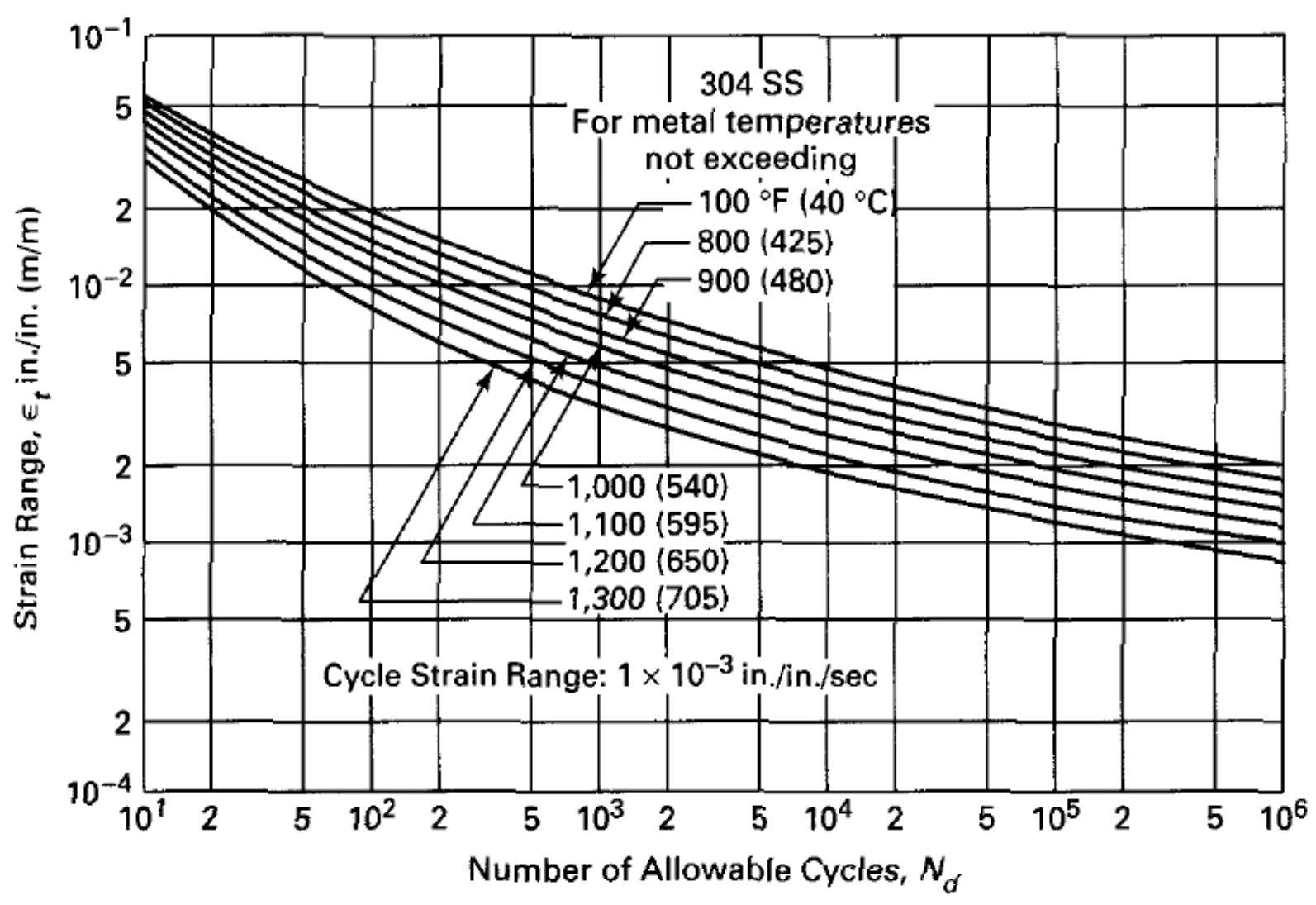




\section{Extrapolation of Section III/NH T-1420-1a curves}

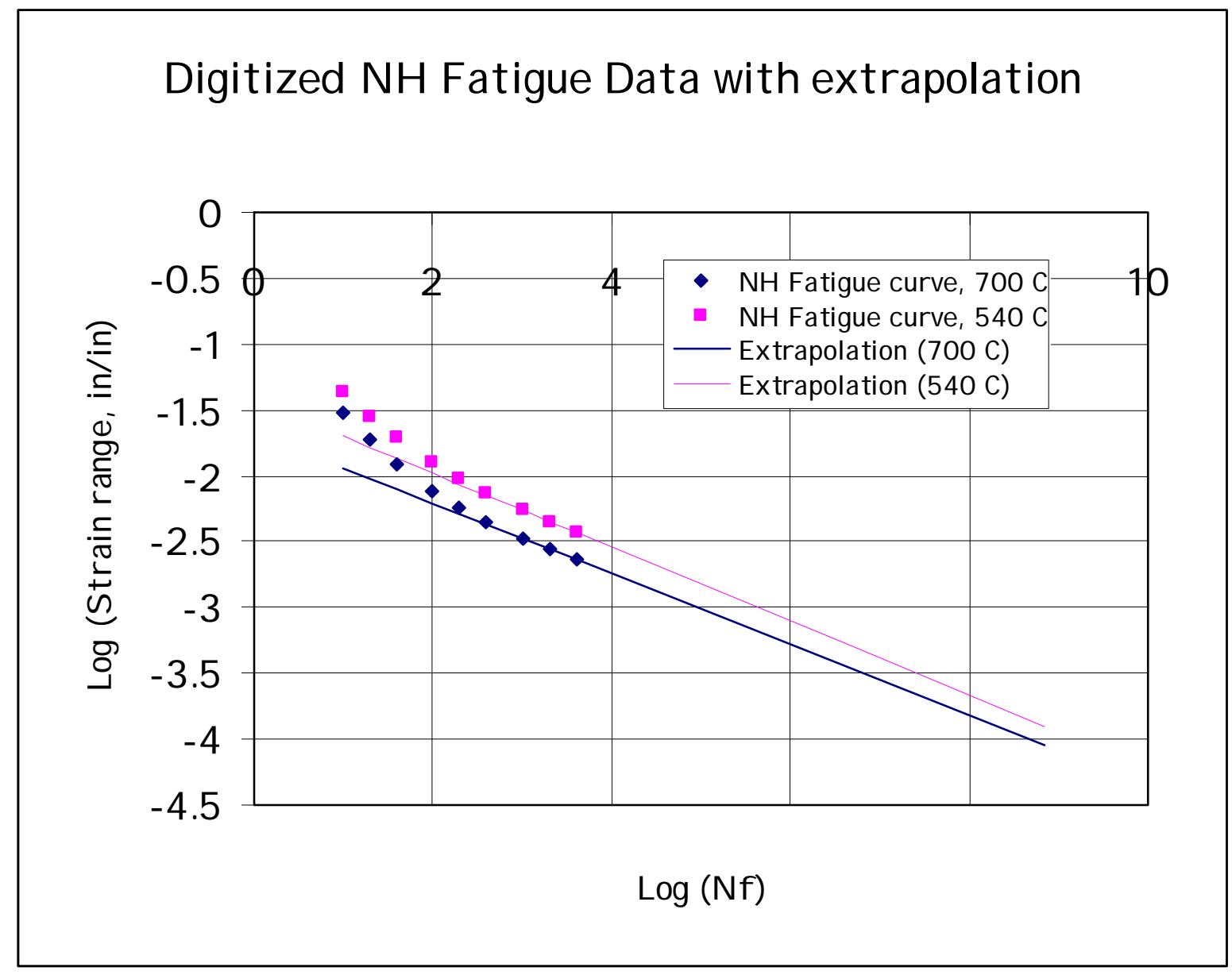




\section{Example of Isochronous Curve from Section III/NH}

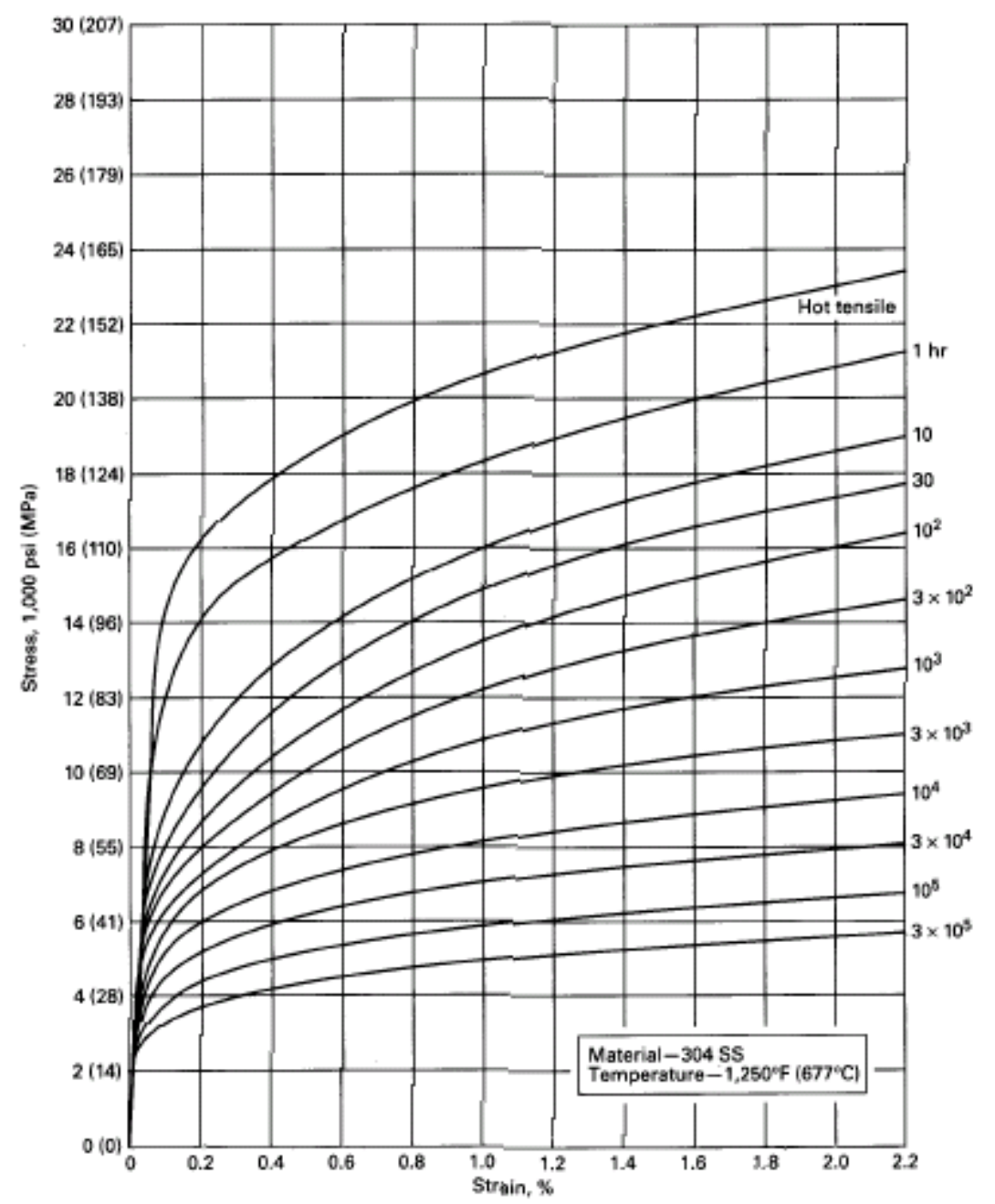

FIG. T-1800-A-10 AVERAGE ISOCHRONOUS STRESS-STRAIN CURVES 
Axisymmetric Simplification of Tapered Sleeve Tee
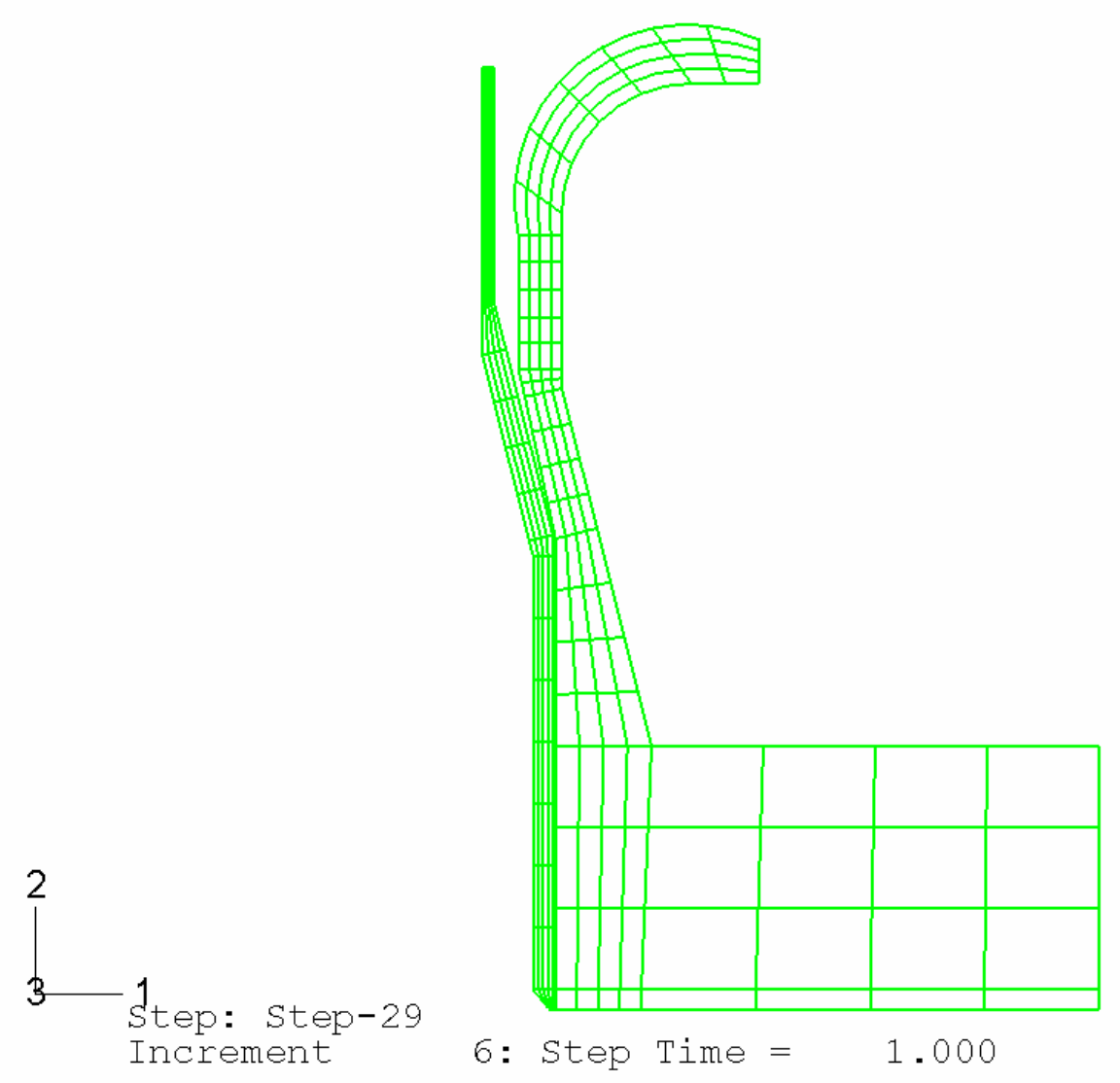
Thermal distribution with heat shield @ 26 seconds

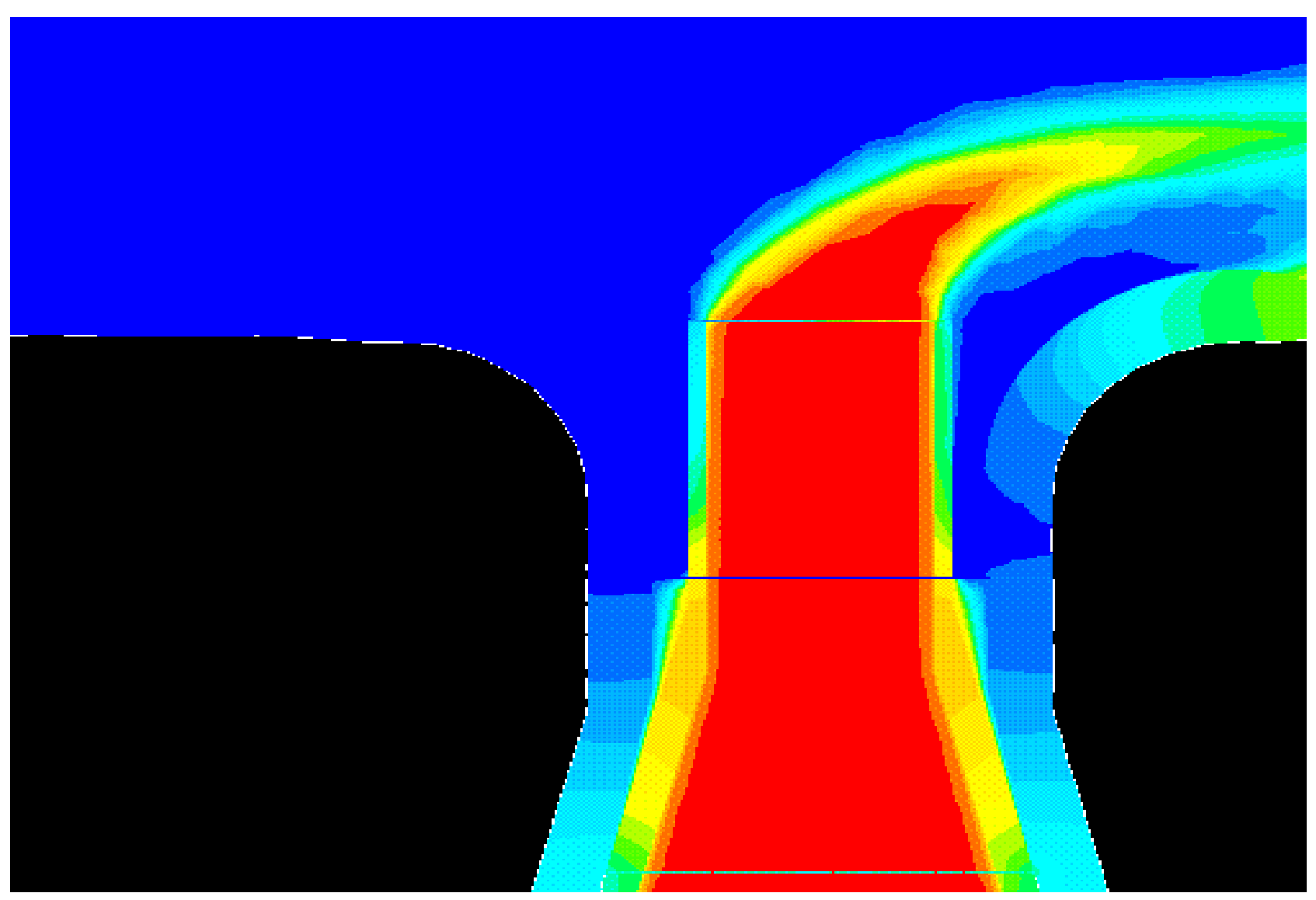


Temperature in Taper at 26 seconds transferred to FE model

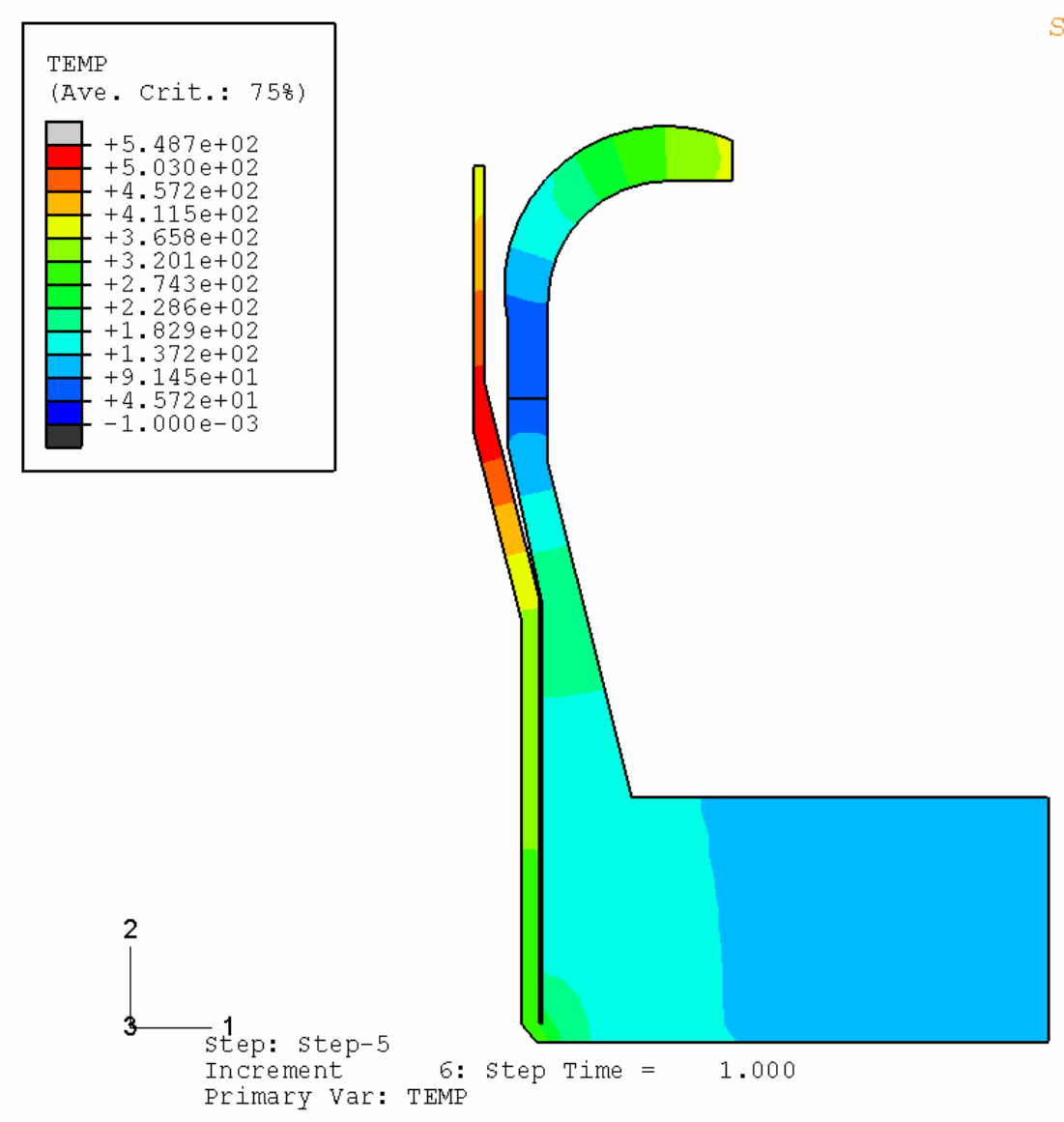




\section{Plastic Strains at 26 seconds}

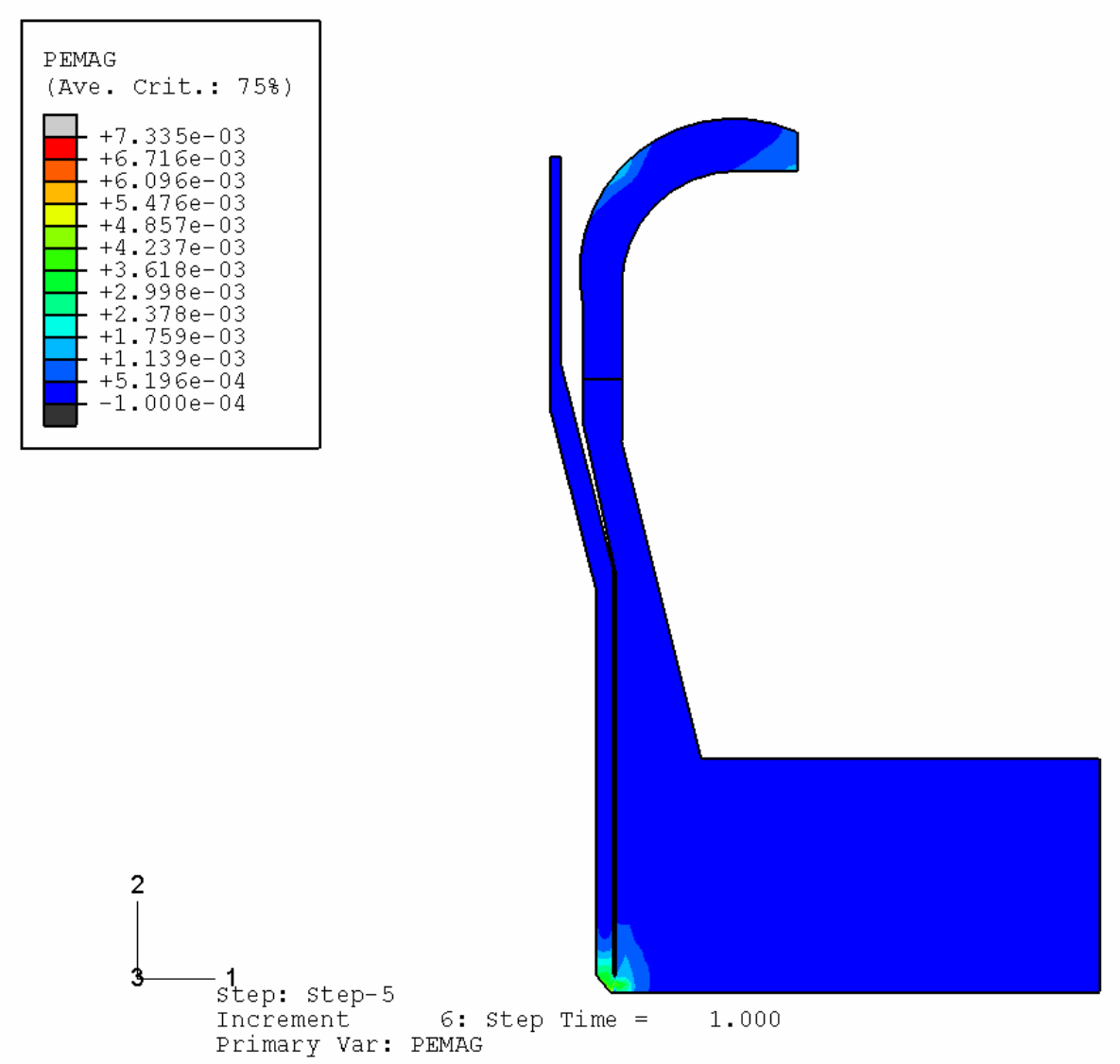

Step: Step-5 Frame: 1 


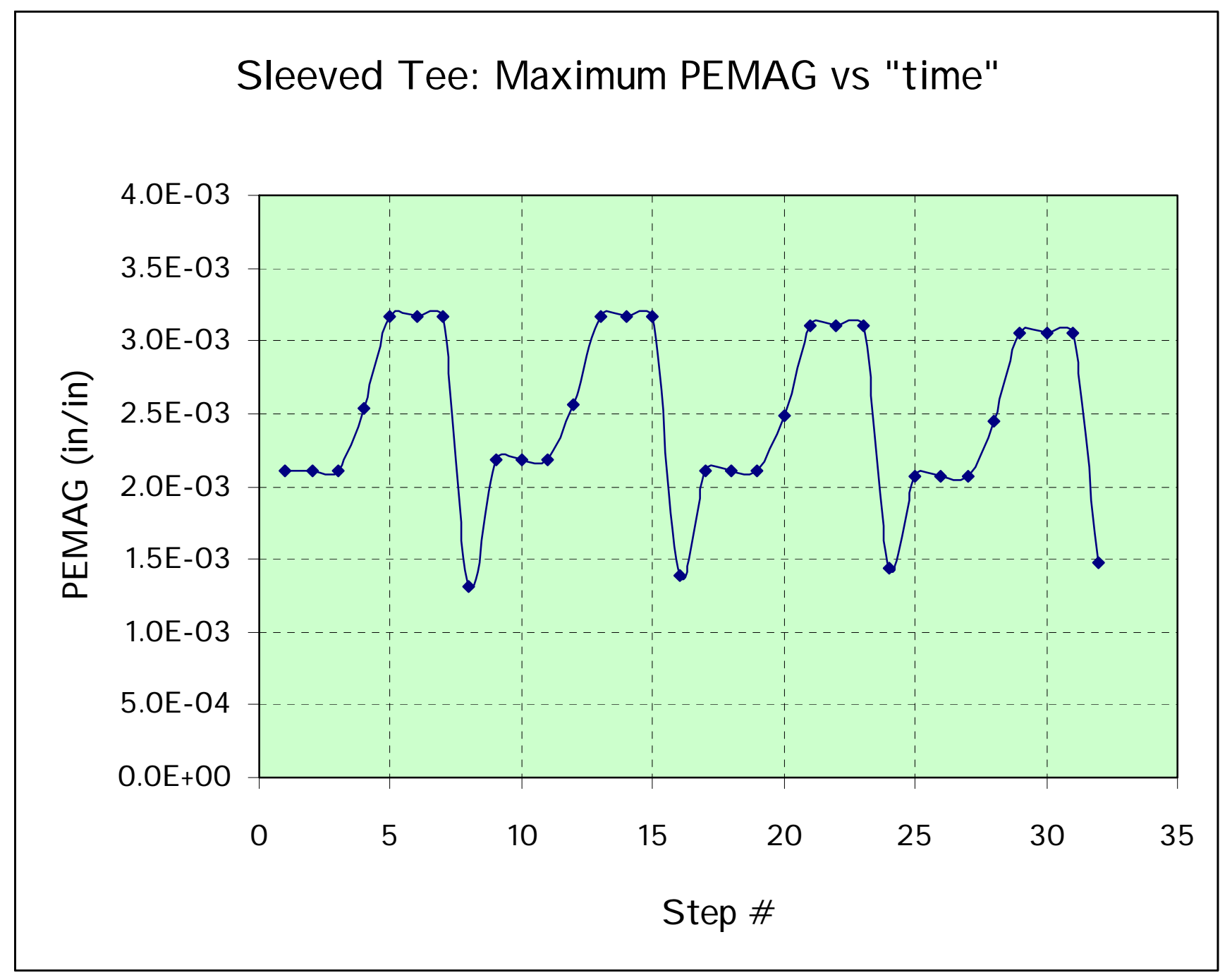




\section{Cumulative Displacement for Two Cycles}

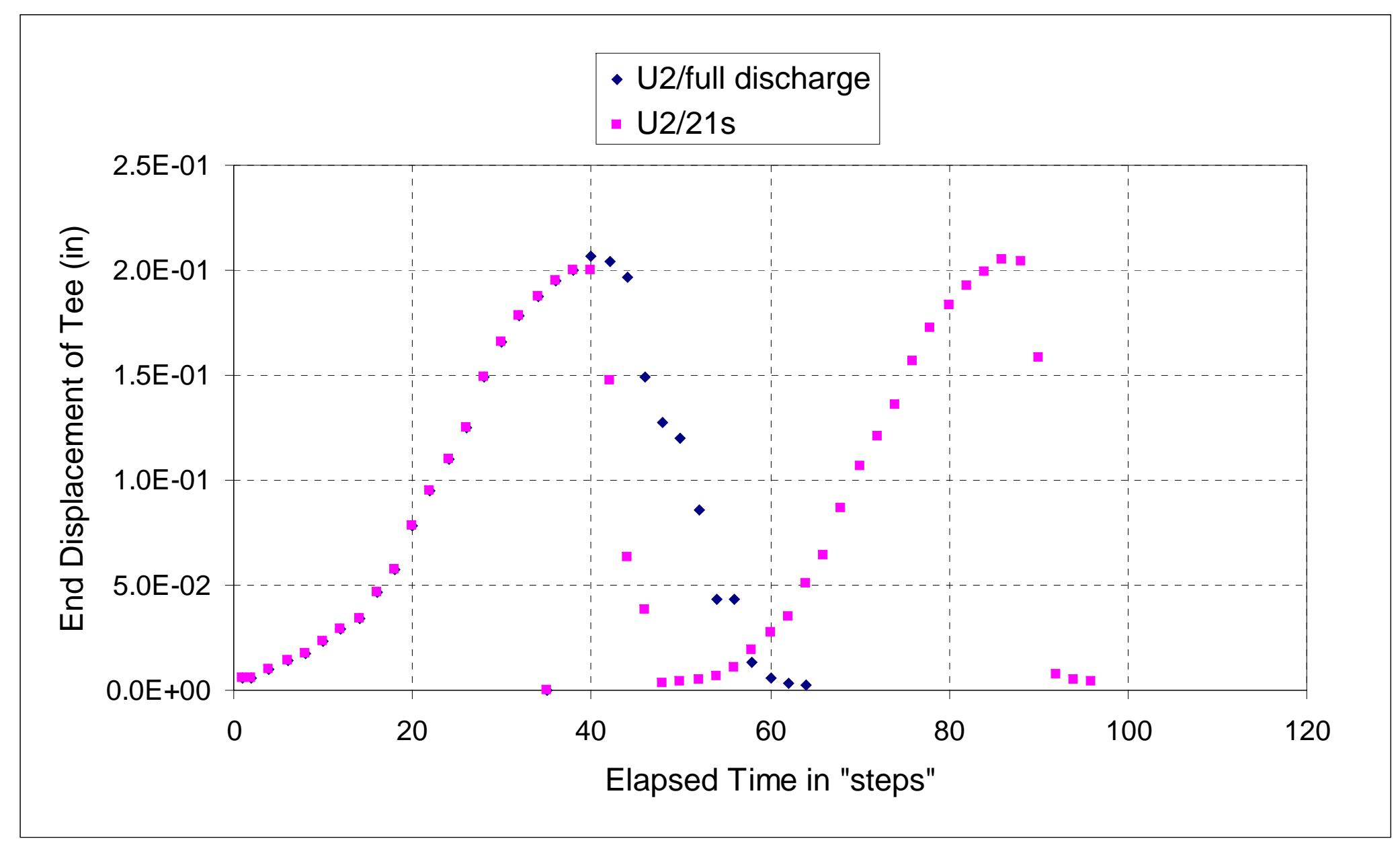




\section{Cyclic Plastic Strains in two cycles}

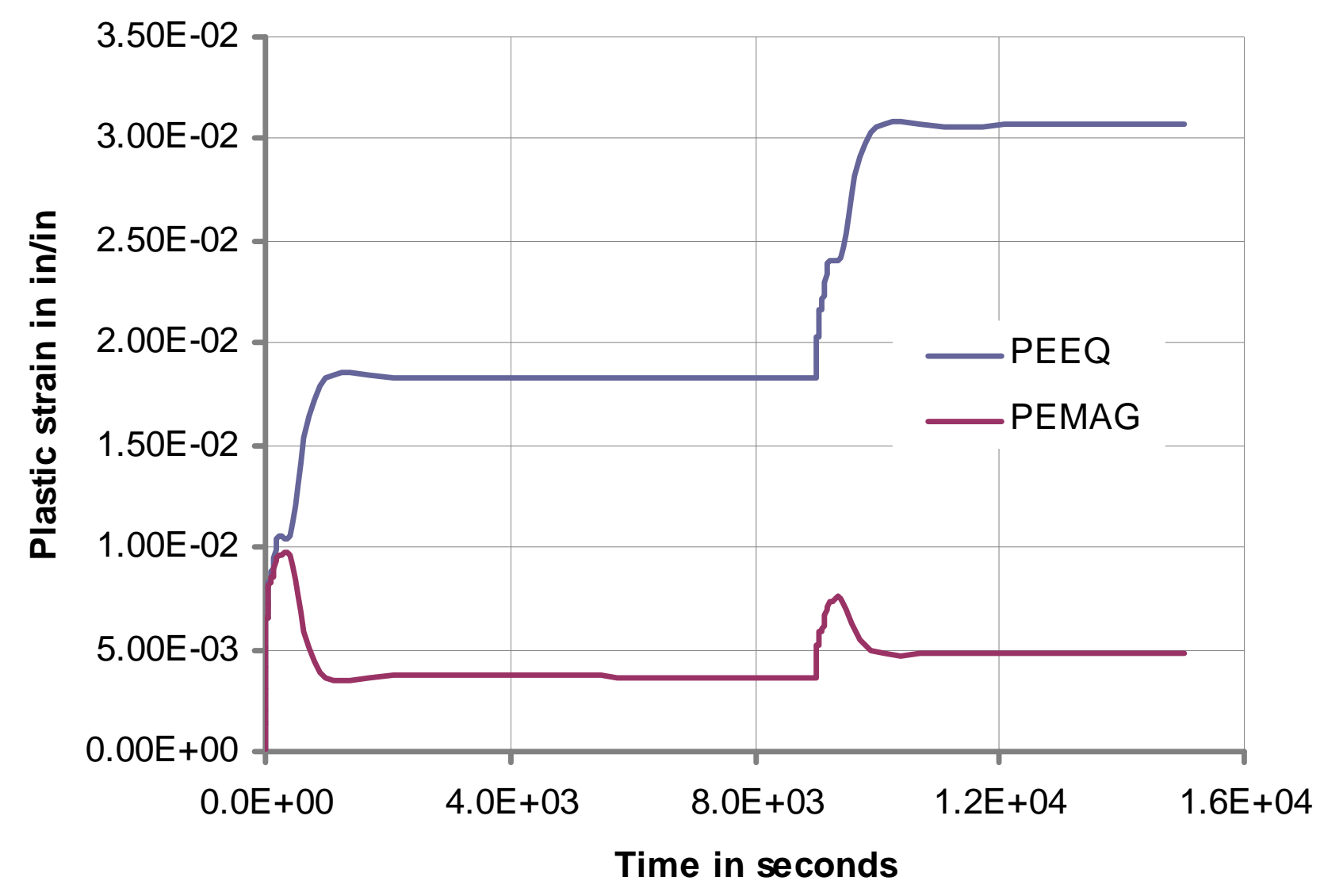




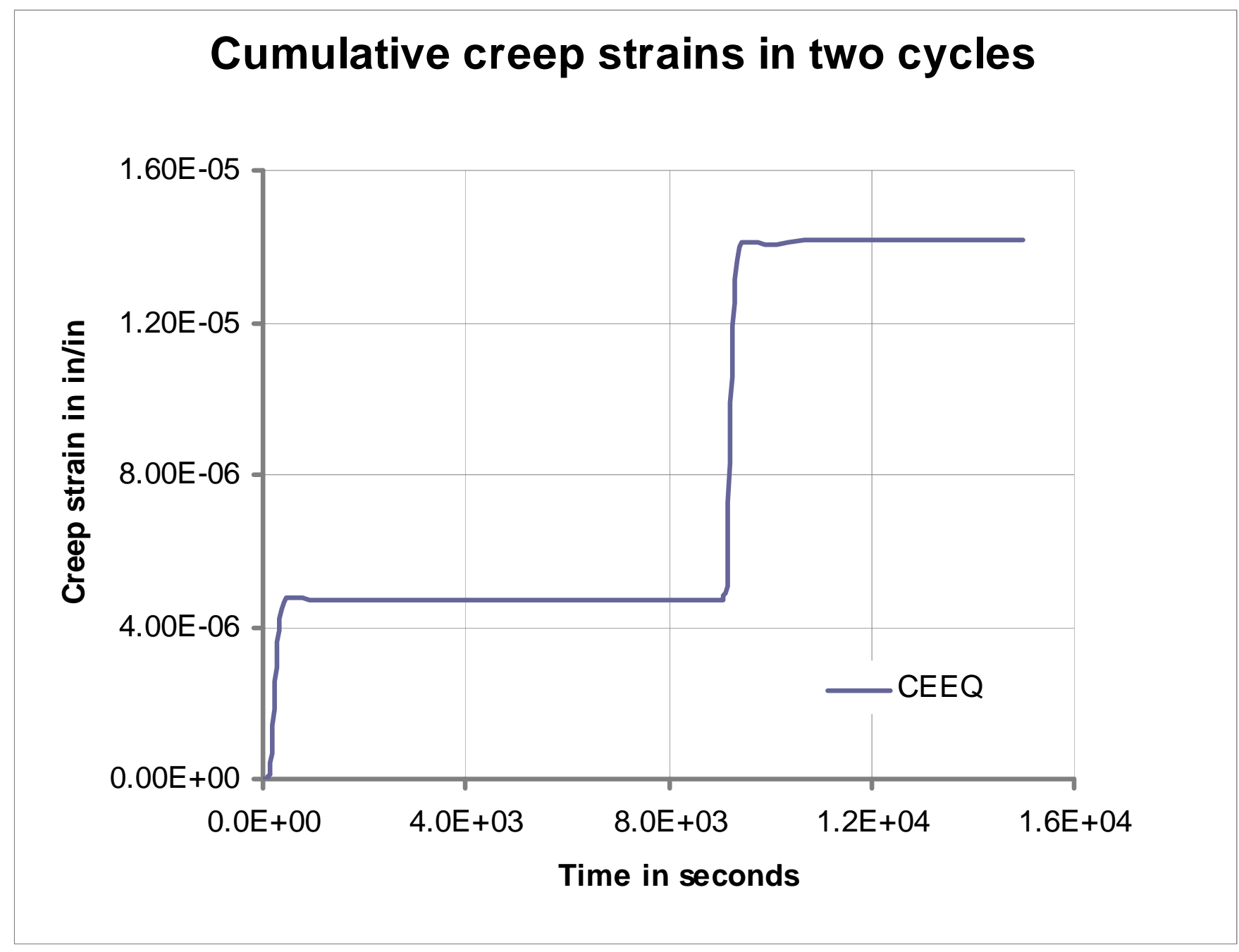




\title{
Task 9: Update and Improve Subsection NH - Simplified Elastic and Inelastic Design Analysis Methods
}

\section{Subtask 9.6 -Recommend Round-Robin Structural Analysis \& Benchmark Problems}

\author{
Gen IV / NGNP Materials Project \\ Submitted to \\ Mr. Jim Ramirez \\ Vice President, Business Development \\ ASME Standards Technology, LLC \\ Three Park Avenue \\ New York, NY 10016 USA
}

By

Advanced Consulting Engineering Services, Inc.

Peoria, IL

On behalf of

Jeries Abou-Hanna

Douglas L. Marriott

Timothy E. McGreevy

June 29, 2009 


\section{TABLE OF CONTENTS}

9.6.1 Objective: Recommendations for Round-Robin Benchmark Problems .......................1

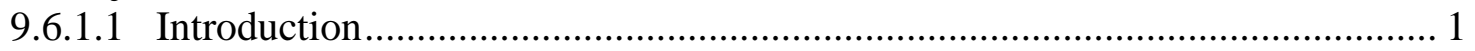

9.6.1.2 Subtask Objective ...................................................................................... 1

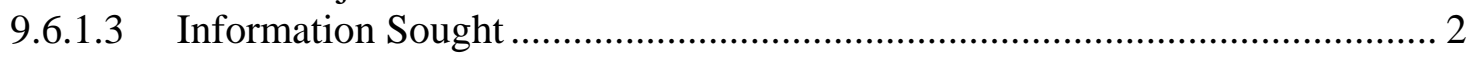

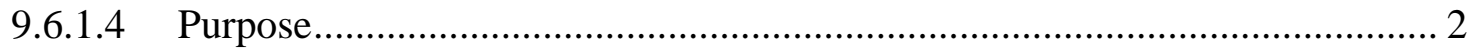

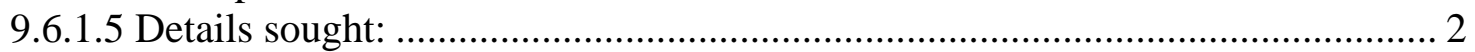

9.6.2 Presentation of Round-Robin Benchmark Problems/Cases:.......................................

9.6.3 Issues and Purpose of Round-Robin Problems:..........................................................

9.6.4 Simplified Methods Recommended for a Round-Robin ............................................

9.6.5 Description of Round-Robin Benchmark Problems ..................................................8

9.6.5.1 C1: Y-Junction \& V Liner Support (V-Ring) .................................................... 9

9.6.5.2 C2: The Harwell Thermal Ratcheting Experiment ........................................... 11

9.6.5.3 \& 9.6.5.4 CP3 \& CP4: Westinghouse/ORNL Validation of Inelastic Analysis

by Full-Scale Component Testing of Nozzles \& Elbows ............................................... 13

9.6.5.5 P5: Analysis of the Type IV Failures of Three Welded Ferritic Pressure

Vessels .................................................................................................................. 16

9.6.5.6 C6: ORNL Creep Ratcheting Studies of Beams, Circular Plates and Stepped

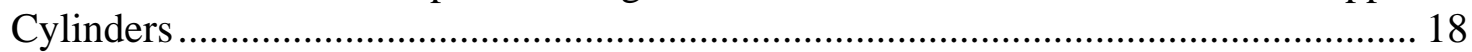

9.6.5.7 P7: Penny and Marriott - Creep of Aluminum Alloy Spherical Pressure Vessel

Nozzle to Rupture ……………………...................................................................... 20

9.6.5.8 CP8: Goodall's Experiments of Simple Plates, Plates with Notches and

Cylinder/Cylinder Intersections .................................................................................... 22

9.6.5.9 CP9: NIL_FFS Project- Experimental and Analytical Investigation of the

Elevated Temperature Performance of a Number of Welded and Unwelded Pressure

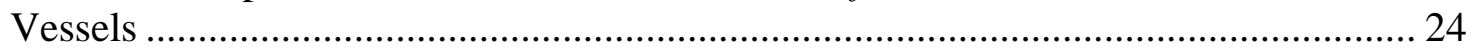

9.6.5.11 P11: Corum-Battiste Experimental and analytical data on a typical nozzle for

LMFBR/CRBRP-IHX.......................................................................................... 28

9.6.5.12 P12: D.L. Marriott - Welded Spacer Connecting Adjacent Legs of a

Serpentine Boiler Tube Platen ........................................................................................ 30

9.6.6 Useful Other Cases and Resources .........................................................................32

9.6.6.1 Use of Isochronous curves to predict inelastic strain: .................................. 32

9.6.6.2 Shakedown, Ratchet and Reversed Plasticity Limits.................................... 32

9.6.6.3 Inelastic Strain in Variable Cycle Loading at Elevated Temperatures:........... 33

9.6.6.4 The ECCC Database of Component Tests and Assessments: ......................... 33

9.6.7 Minimum Material Data Requirements .................................................................33

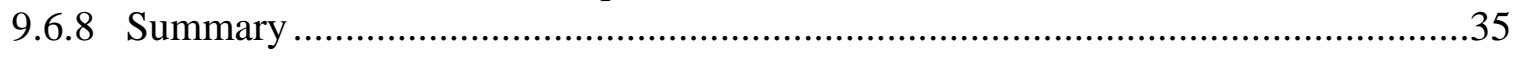

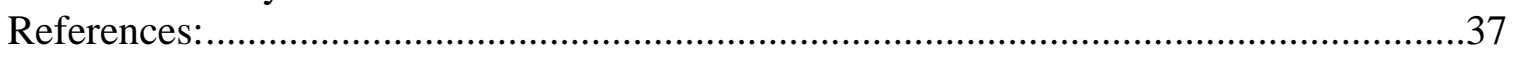

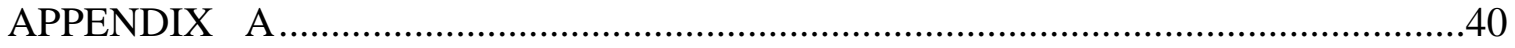




\section{LIST OF TABLES}

Table C1: Round-Robin Benchmark Problem - C1 "Y-Junction Component and PBMR V-Ring”.

Table C2: Round-Robin Benchmark Problem -C2 “The Harwell Thermal Ratcheting Experiment”.

Table CP3: Round-Robin Benchmark Problem - CP3 "Westinghouse-ORNL FullScale Nozzle Testing”.

Table CP4: Round-Robin Benchmark Problem - CP4 "Westinghouse Full-scale Elbow Testing”

Table P5: Round-Robin Benchmark Problem - P5 “Analysis of the Type IV

Failures of Three Welded Ferritic Pressure Vessels"

Table C6 Round-Robin Benchmark Problem-C6 “ORNL Creep Ratcheting Studies of Beams, Circular Plates and Stepped Cylinders"....

Table P7 Round-Robin Benchmark Problem - CP7 "Penny and Marriott - Creep of Aluminum Alloy Pressure Vessel Nozzle to Rupture”.

Table CP8 Round-Robin Benchmark Problem - CP8 “Goodall's Experiments of Plain Plates, Plates with Notches and Cylinder/Cylinder Intersection” .23

Table CP9 Round-Robin Benchmark Problem - CP9 "NIL_FFS Project ....

Table CP10 Round-Robin Benchmark Problem -CP10 “Igaris’ Ratcheting Response of Pipes and Elbows under Cyclic Displacement Controlled Loads”

Table P11 Round-Robin Benchmark Problem - P11 “Corum-Battiste Experimental and analytical data on a typical nozzle for LMFBR/CRBRPIHX”.

Table P12 Round-Robin Benchmark Problem - P12 "D.L. Marriott - Welded spacer connecting adjacent legs of a serpentine boiler tube platen”

Table 9.6.7.1 Minimum material data requirement for primary load analysis, (deformation and/or rupture).....

Table 9.6.7.2 Minimum material data requirement for cyclic load analysis 


\subsubsection{OBJECTIVE: RECOMMENDATIONS FOR ROUND-ROBIN BENCHMARK PROBLEMS}

\subsubsection{Introduction}

An effective Elevated Temperature Design Code (ETD Code) will adequately and safely address relevant failure modes while permitting a range of simplified methods and procedures, which will vary in their degree of complexity, conservatism, and costs in implementing. Two critical aspects of Code development and/or advancement are: i) validation of design criteria with actual structural experiments to ensure safe operation against failure mechanism, and ii) numerical / analytical investigation of existing and future analysis \& design methods and procedures with benchmark problems to arrive at a consensus for approval for use in ETD Code.

Based upon Subtasks 9.2-9.5, recommendations are made herein for future round-robin structural analysis of benchmark problems to support recommended ETD Code simplified analysis methods reported in Subtask 9.5. Minimum material data requirements for implementation of such recommendations are summarized. In conjunction with experimental verification, either with existing results or future component or feature-like testing, any revisions, additions, or alternative design criteria may be validated. Similarly, the minimum data requirements may be used as an initial gap analysis of existing material data complimentary to various simplified methods, either within ASME, International Codes, or similar.

This report includes a statement of the subtask objective and the survey that was sent to industry and government labs in an effort to obtain relevant information and details to align recommendations most appropriate to Code and Code user needs. A description and explanation of the documentation presented in the round-robin cases follows, with tables containing key information about each case. The report also includes a summary of other useful data and studies, and concludes with identification of minimum material data required for various simplified analysis methods.

\subsubsection{Subtask Objective}

As part of an ongoing effort to review and improve the NH ASME Code on elevated temperature design, one of the activities forming part of Task 9 is to recommend roundrobin benchmark exercises to compare simplified analysis methods. The specific objective is to compile a set of standard examples having reliable experimental and/or detailed analytical/numerical results, against which candidate methods of simplified analysis may be compared. Examples with credible and well documented experimental results are preferred as they provide a means of validating results of simplified analysis methods, and preferably, design criteria. 
One part of this effort included gathering information from industry, national labs, and academia on experiments and analytical studies that have been, or are being conducted to study component behavior at elevated temperatures. Of particular interest are numerical predictions of component behavior using detailed inelastic analysis and confirmatory experimental testing.

\subsubsection{Information Sought}

The following information was sought by means of a survey and literature search as they pertain to ETD:

A. experiments on components, and/or

B. detailed component inelastic analysis/simulations;

C. examples of simplified analysis methods applied to ETD, preferably with sufficient supplementary information to allow independent checking by detailed analysis;

D. examples of attempts to apply $\mathrm{NH}$ with outcomes relative to other analysis and/or experiments.

\subsubsection{Purpose}

The purpose of the round-robin proposed would be to assess the ability to adequately and conservatively predict component behavior at elevated temperatures under one or more of the following load conditions:

a. monotonic primary and secondary loads with the aim of capturing/measuring strain, with an emphasis on time dependent inelastic strain, and/or creep rupture;

b. cyclic primary and/or cyclic secondary loads with the aim of capturing/measuring strains/deformations for application to one or more of the following:

i. ratchet limits,

ii. shakedown limits,

iii. reversed plasticity,

iv. elastic and inelastic strain time history.

\subsubsection{Details sought:}

The following details were sought in order to make recommendations that may best serve Code development, the Subgroup on ETD (SG-ETD), and the international ETD community:

1. Details of the geometry of components, sufficient to permit independent modeling and/or repetition of experiments, 
2. Material properties (properties and constitutive models, including but not limited to the following):

a. Elastic modulus and Poisson's ratio, both as a function of temperature

b. Plastic data (curves) as a function of temperature

c. Creep properties (Norton Bailey power law, or Omega model...etc.)

3. Material type, grade, and product form

4. Time history of primary load(s)

5. Time history of secondary load(s)

6. Experimental setup, e.g. support conditions

7. Time history of temperature field

8. Definition of load cycles (primary and/or secondary)

9. Results of the experiment/experimental data:

a. Time history of deflection, strain, and/or loads including measurement locations for use in assessing creep rupture, ratchet limits, shakedown limits and/or inelastic strain limits

b. Any numerical predictions made to verify experimental results (e.g. detailed visco-elasto-plastic FEA results)

\subsubsection{PRESENTATION OF ROUND-ROBIN BENCHMARK PROBLEMS/CASES:}

Information gathered from various sources is presented in the following sections. The cases labeled P pertain to steady (primary) load cases, and those labeled C pertain to cyclic load cases; some of the cases are labeled both $(\mathrm{P} / \mathrm{C})$ if they address both steady and cyclic loading. For each case, a brief description is provided followed by a table that includes:

- References,

- Purpose/Issues,

- Geometry,

- Material Type and Properties,

- Primary Load History,

- Secondary Load History,

- Experimental Setup,

- Measurements,

- Time History of Temperature Field,

- Definition of Load Cycles,

- Results,

- Failure Criterion, and

- Comments.

Table 9.6.2.1 summarizes the round-robin benchmark problems. In that table, the term "Identifier" simply refers to either the author's name and/or the experiment or component name. The second column "Cyclic or Steady" indicates the whether there is a cyclic load, steady load, or both. In some cases, experiments consisted of several loading conditions with some focused on steady loading, such as constant pressure, and others on 
cyclic loading such as cyclic pressure or cyclic thermal conditions. The column labeled "Issues" highlights the major issues the study addresses - either directly or indirectly. For example, elastic follow-up may not necessarily be the objective of the experiment performed, but the column may indicate elastic follow-up to show that the component and loading may be of value as a case for an elastic follow-up study. The remaining columns simply indicate if the documentation/references of the case include experimental, and or detail inelastic and simplified inelastic analysis.

\subsubsection{ISSUES AND PURPOSE OF ROUND-ROBIN PROBLEMS:}

The issues for which Task 9 are focused upon pertain to primary and cyclic deformation. The major challenging issues in predicting deformation are:

1. elastic follow-up

2. creep ductility vs. creep brittle behavior

3. notch and geometric discontinuities

4. nonlinear stress (thermal) gradients

5. stress linearization and classification

6. strain linearization

7. input to additional analysis in ETD, e.g. C-F

Readers should be aware of the following points and observations as they review the cases/problems presented hereafter:

1. The intent was to present and focus on case studies/problems with experimental results, as the ultimate verification; however, some geometric or material details may not be available in the references found and used to provide the case summary. Effort was made to provide a list of references that should contain the missing details needed to reconstruct the problems. Such references may be government lab documents and reports that one should be able to obtain. ASME, including the SGETD, may need to request the release of such materials from DOE and other entities after deciding upon the next course of action.

2. It is believed that even if full geometry, loading, or material details may not be available for past experimental work, some of which was conducted as early as the 1970's, the information provided will still be very useful in helping one develop numerical models that closely replicate the experimental setup, and use the models to investigate and compare full inelastic analysis with simplified analysis results. The authors recommend that a task force be formed to prioritize and select cases to pursue, define and construct the geometry (Pro/E models for example), define the loading cases, define the boundary conditions, and provide a complete and consistent set of material data necessary to pursue full inelastic and simplified analysis in the round robin efforts; this would be necessary to avoid inconsistencies in assumptions regarding boundary conditions, material models and loading conditions in a roundrobin study and to focus on the ability of each simplified method. If interest lies in 
how various modeling assumptions or variations in material properties used impact results, the authors recommend that such efforts are clearly separated from assessment of differences in various simplified methods.

3. Some or most of the problems presented were documented in technical papers or reports that included verifications of simplified analysis methods vs. experimental results or comparison with detailed inelastic analysis. Details of the type of simplified analysis or the verification results were not included or discussed herein since the intent was not to summarize the past work, but the intent was to recommend a set of problems for verification.

4. Since some experimental cases contained weldments, which often are unavoidable in real components, including weldments in round-robin experiments may be necessary.

5. Some of the recommended cases include multitudes of tests (e.g. steady creep, ratcheting, creep relaxation, etc.), and more than one component; therefore, these should be considered as a group of round-robin benchmark problems.

6. A literature survey provided many cases to consider. However, the authors decided to focus in detail on a subset of these cases; the rest of the cases are reviewed briefly and outlined in section 9.6.6 "Useful Other Cases and Resources".

\subsubsection{SIMPLIFIED METHODS RECOMMENDED FOR A ROUND-ROBIN}

The following is a subset of simplified analysis methods recommended for consideration in the round-robin. These methods were discussed in detail in subtasks 9.2-9.4. In the authors' opinion, the round-robin should focus on these since they show a promise of robustness and offer a range of possible effectiveness in terms of effort, applicability, and conservatism in the analysis of components with complex geometry and loading. The recommended Task Force(s) may wish to entertain other methods not listed:

1. ASME Subsection NH Simplified methods (NH and App. T)

2. Reference stress for primary loading

3. Reference stress for cyclic loading

4. Linear Matching Method as discussed in Subtask 9.4. This method predicts elastic, shakedown, plasticity, ratcheting limits as well as inelastic strain under cyclic loading without identification of an elastic core.

5. The Hybrid approach as discussed in Subtask 9.4, which predicts elastic, shakedown, plasticity, ratcheting limits as well as inelastic strain under cyclic loading while identifying an elastic core. 
Table 9.6.2.1 Summary of round-robin benchmark problems.

\begin{tabular}{|c|c|c|c|c|c|c|c|}
\hline $\begin{array}{l}\text { Case \# / } \\
\text { Identifier }\end{array}$ & $\begin{array}{l}\text { Cyclic or } \\
\text { Steady }\end{array}$ & Component & Issues & Experimental & $\begin{array}{c}\text { Full } \\
\text { Inelastic } \\
\text { Analysis } \\
\end{array}$ & $\begin{array}{l}\text { Simplified } \\
\text { Analysis }\end{array}$ & Results \\
\hline $\begin{array}{l}\text { C1/ } \\
\text { Goodall \& } \\
\text { PBMR }\end{array}$ & $\begin{array}{l}\text { Cyclic } \\
\text { mech. and } \\
\text { thermal }\end{array}$ & $\begin{array}{l}\text { Y- Junction \& } \\
\text { PBMR's } \\
\text { V-ring }\end{array}$ & $\begin{array}{l}\text { Cyclic primary, } \\
\text { axisymmetric, cyclic } \\
\text { secondary, notch, possible } \\
\text { nonlinear thermal gradient }\end{array}$ & No & Yes & $\begin{array}{l}\text { Yes in } \\
\text { case of Y- } \\
\text { Junction }\end{array}$ & Inelastic strain \\
\hline $\begin{array}{l}\mathrm{C} 2 / \\
\text { Harwell ratchet } \\
\text { expmt. }\end{array}$ & $\begin{array}{l}\text { Cyclic } \\
\text { thermal } \\
\text { transient }\end{array}$ & Tube & $\begin{array}{l}\text { Axisymmetric, cyclic, } \\
\text { ratchet, large deformation, } \\
\text { nonlinear transient }\end{array}$ & yes & No & Yes & $\begin{array}{c}\text { Ratchet } \\
\text { deformation }\end{array}$ \\
\hline $\begin{array}{l}\text { CP3/ORNL \& } \\
\text { Westinghouse }\end{array}$ & $\begin{array}{l}\text { Cyclic } \\
\text { Thermal }\end{array}$ & $\begin{array}{l}\text { Pressure vessel } \\
\text { nozzle }\end{array}$ & $\begin{array}{l}\text { Thermal trans., axisymm., } \\
\text { creep ratcheting, creep } \\
\text { rupture, elastic followup }\end{array}$ & Yes & Yes & Yes & $\begin{array}{l}\text { Ratchet } \\
\text { deformation, } \\
\text { rupture } \\
\end{array}$ \\
\hline $\begin{array}{l}\text { CP4/ } \\
\text { ORNL \& } \\
\text { Westinghouse }\end{array}$ & $\begin{array}{l}\text { Cyclic and } \\
\text { steady }\end{array}$ & Pipe elbow & $\begin{array}{l}\text { Cyclic thermal transients, } \\
\text { axisymmetric, creep } \\
\text { ratcheting, creep rupture \& } \\
\text { creep relaxation, cyclic } \\
\text { hardening, time dep. plastic } \\
\text { buckling, creep buckling }\end{array}$ & Yes & Yes & Yes & $\begin{array}{l}\text { Strain, creep } \\
\text { ratchet, load } \\
\text { drop in } \\
\text { relaxation }\end{array}$ \\
\hline $\begin{array}{l}\text { P5/ } \\
\text { Budden 's } \\
\text { welded vessels }\end{array}$ & Steady & $\begin{array}{l}\text { Welded pressure } \\
\text { vessels }\end{array}$ & $\begin{array}{l}\text { Reference stress, welds, } \\
\text { creep rupture in weld, } \\
\text { parent metal \& HAZ, crack } \\
\text { growth history }\end{array}$ & Yes & No & Yes & $\begin{array}{l}\text { Rupture time, } \\
\text { crack growth }\end{array}$ \\
\hline $\begin{array}{l}\text { C6/ } \\
\text { ORNL Creep } \\
\text { Ratchet }\end{array}$ & $\begin{array}{c}\text { Cyclic } \\
\text { thermal } \\
\text { shock on } \\
\text { stepped } \\
\text { cyl. }\end{array}$ & $\begin{array}{l}\text { Stepped cylinder, } \\
\text { circular plates \& } \\
\text { beams }\end{array}$ & $\begin{array}{l}\text { geometric discontinuities, } \\
\text { creep ratcheting }\end{array}$ & Yes & Yes & Yes & $\begin{array}{l}\text { Deformations } \\
\text { ratcheting, } \\
\text { creep/fatigue } \\
\text { damage }\end{array}$ \\
\hline $\begin{array}{l}\text { P7/ } \\
\text { Penny-Marriott }\end{array}$ & $\begin{array}{l}\text { Steady } \\
\text { load }\end{array}$ & Sphere-nozzle & $\begin{array}{l}\text { Creep deformation and } \\
\text { creep rupture, axisymm, } \\
\text { geom. discontinuity }\end{array}$ & Yes & Yes & Yes & $\begin{array}{l}\text { deformation, } \\
\text { strains, } \\
\text { rupture life }\end{array}$ \\
\hline $\begin{array}{l}\text { CP8/ } \\
\text { Goodall plates } \\
\text { and cyl.-cyl. }\end{array}$ & $\begin{array}{l}\text { Cyclic } \\
\text { primary } \\
\text { loading }\end{array}$ & $\begin{array}{l}\text { Various: plain and } \\
\text { notched plates, } \\
\text { cylinder-cylinder } \\
\text { vessels } \\
\end{array}$ & $\begin{array}{l}\text { Creep ductility, notch, } \\
\text { elastic followup, creep } \\
\text { rupture }\end{array}$ & Yes & No & $\begin{array}{l}\text { Reference } \\
\text { stress }\end{array}$ & Rupture time \\
\hline
\end{tabular}


Table 9.6.2.1-Cont'd Summary of round-robin benchmark problems.

\begin{tabular}{|c|c|c|c|c|c|c|c|}
\hline $\begin{array}{l}\text { Case \# / } \\
\text { Identifier }\end{array}$ & $\begin{array}{l}\text { Cyclic or } \\
\text { Steady }\end{array}$ & Component & Issues & Experimental & $\begin{array}{c}\text { Full } \\
\text { Inelastic } \\
\text { Analysis } \\
\end{array}$ & $\begin{array}{l}\text { Simplified } \\
\text { Analysis }\end{array}$ & Results \\
\hline $\begin{array}{l}\text { CP9/ } \\
\text { Dutch NIL_FFS } \\
\text { Project }\end{array}$ & $\begin{array}{l}\text { Cyclic and } \\
\text { steady }\end{array}$ & Various & $\begin{array}{l}\text { Notch, elastic follow- } \\
\text { up, creep rupture, } \\
\text { ratcheting, welds, } \\
\text { cracks ..etc. }\end{array}$ & Yes & Yes & Yes & $\begin{array}{c}\text { Deformation, } \\
\text { strains, } \\
\text { rupture life, } \\
\text { creep/fatigue, } \\
\text { creep crack } \\
\text { growth }\end{array}$ \\
\hline $\begin{array}{l}\text { CP10/ } \\
\text { Igari-Ratcheting } \\
\text { Pipes \& Elbows }\end{array}$ & $\begin{array}{c}\text { Cyclic } \\
\text { displacement }\end{array}$ & $\begin{array}{l}\text { Straight pipe and } \\
\text { elbow }\end{array}$ & Ratcheting & Yes & Yes & No & $\begin{array}{c}\text { Strain, } \\
\text { deformation }\end{array}$ \\
\hline $\begin{array}{l}\text { P11/ } \\
\text { Corum_Battiste } \\
\text { sphere/nozzle }\end{array}$ & $\begin{array}{l}\text { Steady and } \\
\text { stepped } \\
\text { pressure }\end{array}$ & $\begin{array}{c}\text { Spherical } \\
\text { pressure vessel } \\
\text { with } \\
\text { axisymmetric. } \\
\text { Nozzle } \\
\end{array}$ & $\begin{array}{l}\text { Creep and creep } \\
\text { rupture, geometric } \\
\text { discontinuity }\end{array}$ & Yes & Yes & No & $\begin{array}{l}\text { Deformation, } \\
\text { rupture time }\end{array}$ \\
\hline $\begin{array}{l}\text { P12/ } \\
\text { Marriott AGR } \\
\text { Spacer }\end{array}$ & Steady & 3-D Spacer & Elastic follow-up, creep & No & Yes & Yes & $\begin{array}{c}\text { Deformation } \\
\text { and strain }\end{array}$ \\
\hline
\end{tabular}




\subsubsection{DESCRIPTION OF ROUND-ROBIN BENCHMARK PROBLEMS}

The round-robin benchmark problems are presented in ascending label order. The cases labeled Cx mean they are cyclic loading, and Px mean primary steady loading, with $\mathrm{x}$ being the case number. The cases labeled CPx mean that they included both cyclic loads and steady primary loads, not necessarily in the same test; however, most of the cases presented include more than one test condition, and some of them include more than one component. There are 12 cases in total. 


\subsubsection{C1: Y-Junction \& V Liner Support (V-Ring)}

This is a numerical investigation that compares the simplified inelastic analysis (ASME Code Case N47 procedure, based on a thin tube formulation) with detailed analysis of gross deformation of a Y-junction (typical configuration found in the CRBRP-IHX) subjected to primary and cyclic secondary loading. The geometry is not specified in the reference cited but should be available in a cited reference [1]. Cumulative inelastic strain and creep-fatigue damage results from simplified analysis method (Case N47 procedure) and detailed inelastic method are compared and tabulated in WRC Bulletin 363 [2].

A substitute case can be found with full detail in a PBMR report obtained in response to the survey conducted and is titled "Core Outlet Hot Gas Duct V-Ring Analysis Report" [3]. The purpose of the analysis was to determine the number of load cycles allowable in the V-shaped liner supports, considering fatigue and ratcheting, when subjected to typical temperature load cycles between $20^{\circ} \mathrm{C}$ and $900{ }^{\circ} \mathrm{C}$ expected during the operational life of the plant. The liners are supported by V-shaped circumferential rings, flexible enough to allow for the temperatureinduced diameter changes between the liner and pressure pipe. This report contains full details of geometry, material and loading and full inelastic analysis results, which can be compared to a variety of simplified analysis predictions. 
Table C1: Round-Robin Benchmark Problem - C1 “Y-Junction Component and PBMR V-Ring”

\begin{tabular}{|c|c|}
\hline $\begin{array}{l}\text { Case \#: C1 } \\
\text { Description }\end{array}$ & $\begin{array}{l}\text { Y- Junction: } \\
\text { [1] Kamal, S.A., Chern, J.M. and, Pai, D.H., “Applications of one-Dimensional Models in Analysis,” ASME } \\
\text { Paper 80-C2/NE-17, presented at Century-2 Pressure Vessel and Piping Conference, San Francisco, CA, Aug. } \\
\text { 1980. } \\
\text { [2] WRC Bulletin } 363 \text { pp42-44. Chapter 6, by Goodall, Dhalla \& Nagata. } \\
\text { This is a numerical investigation that compares the simplified inelastic analysis (ASME Code Case N47 } \\
\text { procedure) based on thin tube formulation, with detailed analysis of gross deformation of a Y-junction } \\
\text { subjected to cyclic primary and secondary loading. This is an axisymmetric problem. } \\
\text { [3] PBMR V-Ring: “Core Outlet Hot Gas Duct V-Ring Analysis Report”, by J. du Plessis et al, Document } \\
\text { Number T100536, April, 2009, PBMR. }\end{array}$ \\
\hline Issues & cyclic primary, cyclic secondary, notch, nonlinear thermal stresses, axisymmetric \\
\hline Purpose & Validate inelastic strain under cyclic primary and secondary loading \\
\hline Geometry of component & Available for PBMR case \\
\hline Material type and properties & Full details given in PBMR report \\
\hline Time history of primary load(s) & Full details given in PBMR report; pressure varies \\
\hline Time history of secondary load(s) & Full details given in PBMR report \\
\hline $\begin{array}{l}\text { Experimental setup, i.e. support } \\
\text { conditions. }\end{array}$ & N/A \\
\hline Measurements & N/A \\
\hline Time history of temperature field & Full details given in PBMR report \\
\hline $\begin{array}{l}\text { Definition of load cycles (primary and/or } \\
\text { secondary) }\end{array}$ & Full details given in PBMR report \\
\hline Results & $\begin{array}{l}\text { Analysis presented compares effective stress, resulting inelastic strain and creep-fatigue damage at two critical } \\
\text { sections at the crotch of the Y-junction. } \\
\text { In PBMR problem, full inelastic finite element analysis results are given. }\end{array}$ \\
\hline $\begin{array}{l}\text { Failure criterion: Total collapse, } 5 \% \\
\text { strain...etc. }\end{array}$ & N/A \\
\hline Comments & $\begin{array}{l}\text { Y-Junction: Refer to reference 6-5 cited in WRC Bulletin } 363 \text { for details [2]. PBMR problem is fully } \\
\text { documented in the PBMR report [3]. }\end{array}$ \\
\hline
\end{tabular}




\subsubsection{C2: The Harwell Thermal Ratcheting Experiment}

Experiments were performed by UKAEA at Risley to simulate deformation in a cylinder subjected to axially moving thermal transient [3]. The cylinder was first heated by radiant heat and then immersed in water at RT, and repeated for 15 cycles. Detail of temperature ranges can be found in pp 48-49 [3]. A similar experiment, known as the Saclay experiment, was briefly reported in [3] as well, pp 48-49, but without sufficient detail. However, the bulletin contains reference citations for the Saclay experiment, where details may be acquired [4].

A similar investigation was reported by Japanese researchers [5]. Igari, et al. developed experiments to investigate thermal ratcheting with thermal gradients across the tube section that are step, linear and nonlinear, with and without hold time. They compared the results to simplified elastic methods. This is a very useful case since it includes nonlinear thermal gradients. 
Table C2: Round-Robin Benchmark Problem -C2 “The Harwell Thermal Ratcheting Experiment"

\begin{tabular}{|c|c|}
\hline $\begin{array}{l}\text { Case \#: C2 } \\
\text { Description }\end{array}$ & $\begin{array}{l}\text { [1] WRC Bulletin 363, pp44-48. Chapter 6. By Goodall, Dhalla \& Nagata. } \\
\text { [4] Bell, R.T. "Interim Results from CTS/Harwell Ratcheting Experiments on Thin Cylinders,” ND-M-352(R) Risley } \\
\text { Nuclear Power Development, April 1978. } \\
\text { This is an investigation that compares the simplified inelastic analysis based on thin tube formulation, with experimental } \\
\text { results of ratchet deformation of tube under severe axial thermal wave. } \\
\text { [5] T. Igari, "Mechanism-Based Evaluation of Thermal Ratcheting due to Traveling Temperature Distribution,” Trans. } \\
\text { ASME, Vol. 122, May 2000, pp. 130-138. }\end{array}$ \\
\hline Issues & Cyclic thermal transients, thermal membrane stresses, ratcheting, large deformations \\
\hline Purpose & Compare ratcheting deformation results of experiment with those of simplified methods \\
\hline Geometry of component & The tube geometry is provided in [5], pg 45. Tube thickness $=0.015$ inch, diameter $=5.5$ inch and length $=14$ inches. \\
\hline Material type \& properties & $\begin{array}{l}\text { Reference 6-10 in WRC Bulletin } 363 \text { should have material data: Bell, R.T. “Interim Results from CTS/Harwell } \\
\text { Ratcheting Experiments on Thin Cylinders,” ND-M-352(R) Risley Nuclear Power Development, April } 1978 .\end{array}$ \\
\hline Time history of primary load(s) & No primary load \\
\hline Time history of secondary load(s) & Cyclic thermal transient described above \\
\hline $\begin{array}{l}\text { Experimental setup, i.e. support } \\
\text { conditions. }\end{array}$ & $\begin{array}{l}\text { Tube heated from RT to a uniform high temperature, then immersed gradually in RT water; process repeated } 15 \text { times, } \\
\text { causing ratcheting. Exact details of thermal cycle are found in cited references. }\end{array}$ \\
\hline Measurements & Radial deformation/swelling \\
\hline Time history of temperature field & Thermal wave described above. \\
\hline $\begin{array}{l}\text { Definition of load cycles (prim. } \\
\text { and/or secondary) }\end{array}$ & Thermal wave described above. Maximum temperature gradient is 530C and 450C. \\
\hline Results & $\begin{array}{l}\text { Plastic ratchet predictions are compared to experiment results. The comparison is available graphically in the cited } \\
\text { reference. Prediction did not include creep, but only plasticity, resulting in under prediction of deformation. }\end{array}$ \\
\hline $\begin{array}{l}\text { Failure criterion: Total collapse, } \\
5 \% \text { strain...etc. }\end{array}$ & Excessive ratcheting \\
\hline Comments & \\
\hline
\end{tabular}




\subsubsection{3 \& 9.6.5.4 CP3 \& CP4: Westinghouse/ORNL Validation of Inelastic Analysis by Full-Scale Component Testing of Nozzles \& Elbows}

This experiment is very comprehensive; it includes numerous load conditions and failure modes: rupture, buckling, creep buckling, ratcheting and creep cracking in welds. This is an excellent case to include in the round-robin; the paper clearly indicates that full details are available in DOE reports. The testing was performed at Westinghouse Advanced Energy Systems Division [6].

Full scale prototype elbow and pressure vessel nozzle components were tested under primary and cyclic load conditions. Elbow tests included short time and creep buckling conditions. Rotation of the elbow was measured as the in-plane moment was increased in case of short term buckling test, and kept constant in case of creep deformation or creep buckling test. A relaxation test was conducted by holding the end rotation constant and monitoring the moment. Creep ratcheting tests were conducted by applying pressure and monitoring the hoop strain at mid elbow.

Three inlet nozzles were located along a cylindrical pressure circumference, equidistant. A fourth outlet nozzle was installed at one end of the vessel. Primary pressure load testing was performed for creep deformation measurements. Temperature, strain, pressure and gas flow rate measurements were made. Thermal cycles were imposed and included dwell times in the 150200 hour range. The vessel was uniformly heated to 566C, while nozzles were subjected to several downshocks.

Full inelastic analyses were conducted and results compared with experiments. Full inelastic analyses and simplified analyses for assessing creep deformation were conducted using an idealized thick cylinder assumption and are described in detail in [2] on pp 34-41. Creep rupture cracks formed in the weld areas, and inelastic analysis were conducted to determine the effects of residual stresses and material strength differences on rupture. 
Table CP3: Round-Robin Benchmark Problem - CP3 “Westinghouse-ORNL Full-Scale Nozzle Testing”

\begin{tabular}{|c|c|}
\hline $\begin{array}{l}\text { Case \#: CP3 } \\
\text { Description }\end{array}$ & $\begin{array}{l}\text { [6] D.S. Griffin, A.K. Dhalla, W.S. Woodward, "Validation of Inelastic Analysis by Full-Scale Component } \\
\text { Testing”, 42/Vol. 109, Feb 1987. Compares theoretical and experimental results of full scale components } \\
\text { tested at ET to validate inelastic analysis methods, material models and design limits. } \\
\text { Nozzle: creep ratcheting \& weld cracking and thermal striping fatigue. Work funded by DOE \& full } \\
\text { report is available. } \\
\text { [2] WRC Bulletin } 363 \text { - May 1991, Recommended Practices in ETD: A compendium of Breeder Reactor } \\
\text { Experiences (1970-1987), Vol. II - Preliminary Design and Simplified Methods, Ch. 6.0 Simplified } \\
\text { Methods by I.W. Goodall, A.K. Dhalla and T. Nagata. } \\
\text { [7] W.S. Woodward and A.K. Dhalla, “Evaluation of Inelastic Strain Accumulation for the FFTF/IHX } \\
\text { Nozzle Proof Test,” Westinghouse Electric Corporation, Advanced Reactors division, Report \#WARD- } \\
\text { HT-94000-10 prepared for USDOE under Contract \#DE-AMO2-76CH94000, September 1980. } \\
\text { [8] W.K. Sartory, Analytical Investigation of the Applicability of Simplified Ratcheting and Creep-Fatigue } \\
\text { Rules to LMFBR Component Geometries - Two Dimensional Axisymmetrical Structures. ORNL/TM- } \\
\text { 5616, December 1976. }\end{array}$ \\
\hline Issues & Cyclic thermal transients, axisymmetric loading and geometry, creep ratcheting and creep rupture \\
\hline Purpose & $\begin{array}{l}\text { To validate simplified rules contained in ASME CC 1592-3 (precursor NCC N-47 and Section } \\
\text { III/Subdivision NH) for evaluation of shakedown and ratcheting under constant internal pressure and a } \\
\text { cyclically varying through-thickness temperature gradient. The intent was to extend the applicability of } \\
\text { rules written originally for a simple Bree-type tube to more complex geometries. }\end{array}$ \\
\hline Geometry of component & FFTF-IHX Nozzle/pressure vessel: three radial inlet nozzles on a cylindrical vessel. \\
\hline Material type \& properties & $\begin{array}{l}304 \text { SS. Extensive description of material properties relevant to cyclic load analysis provided, including } \\
\text { elastic/plastic behavior and creep. Quantitative data drawn from [9] RTD Standard F9-5T. }\end{array}$ \\
\hline Time history of primary load(s) & Constant pressure \\
\hline Time history of secondary load(s) & Thermal down shock. \\
\hline Experimental setup, i.e. support conditions. & DOE report has full details, according to paper. \\
\hline Measurements & $\begin{array}{l}\text { Measured temp., pressure, gas flow rate \& residual strain. Maximum creep ratchet strain identified at } \\
\text { knuckle region at inside surface. }\end{array}$ \\
\hline Time history of temperature field & Thermal downshocks \\
\hline Definition of load cycles (prim/ secondary) & DOE report has full details. Scanned version of full report available on-line or on request \\
\hline Results & $\begin{array}{l}\text { Experiments: Maximum creep ratchet strain identified at knuckle region by inelastic analysis. Full } \\
\text { Inelastic analyses and simplified analyses idealizing the nozzle as a uniform thickness thick cylinder were } \\
\text { conducted and compared with experiments. See [2] pp 34-40. DOE report has full details, according to } \\
\text { paper. }\end{array}$ \\
\hline Failure criterion: Total collapse, 5\% strain...etc. & N/A except for investigation of rupture cracks in weld area. \\
\hline Comments & $\begin{array}{l}\text { Creep rupture cracks showed up in weld area and investigated; inelastic analysis were performed to predict } \\
\text { effect of residual stresses and material strength difference on rupture; conclusion is residual stresses had no } \\
\text { impact, but weld lower creep rupture strength explained the cracking. Excellent problem to study. DOE } \\
\text { report has full details, according to paper. }\end{array}$ \\
\hline
\end{tabular}


Table CP4: Round-Robin Benchmark Problem - CP4 “Westinghouse Full-scale Elbow Testing”

\begin{tabular}{|c|c|}
\hline $\begin{array}{l}\text { Case \#: CP4 } \\
\text { Description }\end{array}$ & $\begin{array}{l}\text { [6] D.S. Griffin, A.K. Dhalla, W.S. Woodward, "Validation of Inelastic Analysis by Full-Scale Component } \\
\text { Testing”, 42/Vol. 109, Feb } 1987 . \\
\text { Compares theoretical and experimental results of full scale components tested at ET o validate inelastic } \\
\text { analysis methods, material models and design limits. Elbow: plastic \& creep buckling creep ratcheting \& } \\
\text { creep relaxation. Work funded by DOE \& full report should be available. }\end{array}$ \\
\hline Purpose & Compare simplified methods with both experimental and inelastic analysis. \\
\hline Geometry of component & Piping elbow: seamless elbow, 16 inch diam.; thickness = 0.4 inch; welded to tangent straight pipes \\
\hline Material type and properties & $\begin{array}{l}\text { Models used: elastic; elast-plastic; creep behavior, 593C; creep equations included primary \& secondary } \\
\text { creep terms. } \\
\text { For ratcheting: used linear kinematic hardening with ORNL’s } 10^{\text {th }} \text { cycle hardening rule. }\end{array}$ \\
\hline Time history of primary load(s) & $\begin{array}{l}\text { Elbow: TimeDep. Plastic buckling: Increased In plane moment on one end to full collapse. } \\
\text { Elbow: Creep Deformation: Constant In plane moment on one end. } \\
\text { Elbow: Creep Ratcheting \& Creep Relaxation: Constant Pressure. } \\
\text { Elbow: Creep Buckling: constant closing In-plane moment; } 90 \% \text { of instantaneous buckling moment, } \\
\text { with objective of buckling in } 2000 \text { hrs \& about } 506 \text { degree rotation }\end{array}$ \\
\hline Time history of secondary load(s) & $\begin{array}{l}\text { Elbow: Creep Ratcheting \& Creep Relaxation: Closing/opening elbow rotation. 5-9 cycles with } 160 \mathrm{hr} \\
\text { dwell at closing moment. }\end{array}$ \\
\hline Experimental setup, i.e. support conditions. & $\begin{array}{l}\text { Elbow: One side is fixed, and the other side is subjected to in-plane moment for buckling, or rotation for } \\
\text { creep ratcheting tests. }\end{array}$ \\
\hline Measurements & $\begin{array}{l}\text { Elbow: Time Dep. Plastic Buckling\& Creep deformation: Measured elbow rotation. } \\
\text { Elbow: Creep Ratcheting \& Creep Relaxation: Measured hoop strain at mid elbow near the crown. }\end{array}$ \\
\hline Time history of temperature field & Elbow: Time Dep. Plastic Buckling: constant - 2 cases: room temp. and 593C. \\
\hline $\begin{array}{l}\text { Definition of load cycles (primary and/or } \\
\text { secondary) }\end{array}$ & $\begin{array}{l}\text { Elbow: Time Dep. Plastic Buckling: increasing moment until collapse } \\
\text { Elbow: Time Dep. Creep Deformation: constant moment that does not result in plastic or creep buckling } \\
\text { for } 2761 \text { hours to allow significant secondary creep. }\end{array}$ \\
\hline Results & $\begin{array}{l}\text { Experiments: } \\
\text { Elbow: Collapse Load in buckling, hoop strain at mid elbow in creep ratcheting, moment during } \\
\text { relaxation. } \\
\text { Full Inelastic analyses compared with experiments. }\end{array}$ \\
\hline Failure criterion: Total collapse, $5 \%$ strain...etc. & Elbow: time dep. Plastic buckling: full collapse. Measured elbow rotation. \\
\hline Comments & Excellent problem to study \\
\hline
\end{tabular}




\subsubsection{P5: Analysis of the Type IV Failures of Three Welded Ferritic Pressure Vessels}

This experiment is concerned with creep rupture of and crack growth history in welded pressure vessels that have different weld configurations with different pre-existing residual stress history and creep [10]. The experiments were performed in the 1980s by the former Central Electricity Generating Board (CEGB) to validate ETD assessment methods. Three vessels differed in size, wall thickness and weld configurations. All vessels had end cap welds, and pipe-to-pipe welds (the vessel was made up of several segments of piping welded together).

The loads consist of primary constant pressure that changed from one hold period to another but remained constant during hold time. A reference stress approach was used to predict the rupture time in the parent metal, weld metal and HAZ. The paper contains crack growth data that enabled the evaluation of crack growth history. It appears that the temperature was kept constant during hold periods, and there is no indication that significant thermal gradients existed. 
Table P5: Round-Robin Benchmark Problem - P5 “Analysis of the Type IV Failures of Three Welded Ferritic Pressure Vessels”

\begin{tabular}{|c|c|}
\hline $\begin{array}{l}\text { Case \#: P5 } \\
\text { Description }\end{array}$ & $\begin{array}{l}\text { [10] P.J. Budden, “Analysis of the Type IV failures of three welded ferritic pressure vessels”, Elsevier, } \\
\text { Int'l Journal of PVP, Vol. 75, Issue 6, 1998, pp 509-519. } \\
\text { Large scale pressure vessel creep tests were performed to validate ETD assessment methods. The paper } \\
\text { describes analyses of } 3 \text { tests performed in the 1980’s by Marchwood Engineering Labs (MEL). The paper } \\
\text { compares experimental creep rupture conditions with R5 predictive methods as well as crack growth } \\
\text { history. One vessel had a preexisting circumferential machined crack, and the other two were defect free. }\end{array}$ \\
\hline Issues & Creep rupture, weldments, and crack creep growth, Reference Stress applied to Cracked component. \\
\hline Purpose & $\begin{array}{l}\text { To use the experimental data and R5 predictions to compare with other ETD simplified methods for } \\
\text { predicting creep rupture and crack growth in weld areas. The paper uses reference stress approach to } \\
\text { predict creep rupture time. }\end{array}$ \\
\hline Geometry of component. & $\begin{array}{l}\text { Vessel geometry is provided in reference above. OD range of } 175-258 \mathrm{~mm} \text {, with mean radius to wall } \\
\text { thickness ratio ranging from } 2.4 \text { to } 8.7 \text {. Different circumferential weld configurations in each vessel: } \\
\text { MMA end cap closure welds and pipe-to-pipe welds. One vessel had a preexisting circumferential crack, } \\
\text { and the other two were defect free. One vessel had old welds subjected to various creep histories that are } \\
\text { documented in the paper. }\end{array}$ \\
\hline Material type and properties & $\begin{array}{l}\text { Low alloy Ferritic steel } 0.5 \mathrm{Cr}-0.5 \mathrm{Mo}-0.25 \mathrm{~V} \text {; weld- 2Cr-1Mo. In one case welds were post weld heat } \\
\text { treated, but not in the other two vessels (details in paper). Measured residual stresses are provided. } \\
\text { Rupture data is given for weld and parent metals }\end{array}$ \\
\hline Time history of primary load(s) & Constant pressure but varied from one hold period to another. \\
\hline Time history of secondary load(s) & NA \\
\hline Experimental setup, i.e. support conditions & Details in reference \\
\hline Measurements & Cracks inspected \\
\hline Time history of temperature field & $\begin{array}{l}\text { Constant for long hold periods 10,000 hrs- 50,000 hrs. Temperature varied from one hold period to } \\
\text { another. }\end{array}$ \\
\hline $\begin{array}{l}\text { Definition of load cycles (primary and/or } \\
\text { secondary) }\end{array}$ & NA \\
\hline Results & $\begin{array}{l}\text { Steam Leakage in cracks at 53,746 hrs in FM2A Vessel case (preexisting crack) } \\
\text { Reference stress rupture times are given for parent and weld metals as well as HAZ. Also time for crack } \\
\text { growth Type IV is given. All provided for the three vessels. } \\
\text { Predicted and measured crack depths vs. time are given in the paper }\end{array}$ \\
\hline Failure criterion: Total collapse, 5\% strain...etc. & Leak \& progressive crack growth \\
\hline Comments & $\begin{array}{l}\text { Excellent for weld analysis and crack growth, predictions of rupture times successful w/out considering } \\
\text { cracks }\end{array}$ \\
\hline
\end{tabular}




\subsubsection{C6: ORNL Creep Ratcheting Studies of Beams, Circular Plates and Stepped Cylinders}

Experimental and analytical studies of the ratcheting behavior of several simple component geometries were conducted [11-13]. The three reports describe a single program carried out at ORNL involving a number of detailed experiments on simple components subjected to variable load.

ORNL 5616 [11] describes an analytical study of nine axisymmetric component problems under cyclic mechanical and thermal loading. The component geometries consisted of a notched cylinder, stepped-wall cylinder and built-in end on a cylinder. Other variations consisted of load histories including axial temperature variation and axial variation in a radial thermal gradient. The thermal loading was imposed by transient fluid conditions and made no $a$ priori assumptions regarding temperature or thermal stress distributions. Ratcheting deformations, creep and creep/fatigue damage calculations were carried out by detailed FEA and compared with simplified methods which were then current in CC 1592-3 (forerunner to NH).

ORNL 5626 [12] describes an experimental and computational study of a stepped cylinder under repeated thermal shock combined with sustained internal pressure. This is an experimental complement to the theoretical studies described in ORNL 5616 above.

ORNL/TM-7085 [13] is continuation of the experimental program on beams and plates and involving a simple, but nonaxisymmetric beam in bending. The three reports together provide excellent benchmark material for validation of design analysis techniques. 
Table C6 Round-Robin Benchmark Problem-C6 “ORNL Creep Ratcheting Studies of Beams, Circular Plates and Stepped Cylinders"

\begin{tabular}{|c|c|}
\hline $\begin{array}{l}\text { Case \#: C6 } \\
\text { Description }\end{array}$ & $\begin{array}{l}\text { [11] W.K. Sartory, Analytical Investigation of the Applicability of Simplified Ratcheting and Creep- } \\
\text { Fatigue Rules to LMFBR Component Geometries - Two Dimensional Axisymmetrical Structures. } \\
\text { ORNL/TM-5616, December 1976. } \\
\text { [12] W.K. Sartory and R.L. Battiste, "Inelastic Ratcheting Analysis of the 21/4 Cr 1Mo Steel Stepped-wall } \\
\text { Region of Specimen TTT-3, ORNL-5626, April } 1980 . \\
\text { [13] R.L. Battiste and M. Richardson, "Elevated Temperature Benchmark Tests of 21/4Cr 1Mo Steel } \\
\text { Simply supported Beams and a Circular Plate subjected to Time-Varying Loadings, ORNL/TM-7085, } \\
\text { May 1980. } \\
\text { * ORNL 5626 describes an experimental and computational study of a stepped cylinder under repeated } \\
\text { thermal shock combined with sustained internal pressure. } \\
\text { * ORNL/TM-7085 contains descriptions of experimental results only, on simple beams and circular plates. }\end{array}$ \\
\hline Issues & Creep ratcheting, inelastic strain, notch, elastic followup \\
\hline Purpose & To validate various simplified methods. \\
\hline Geometry of component & Simple beam and circular plates \\
\hline Material type and properties & $\begin{array}{l}\text { Extensive description of all material properties relevant to cyclic load analysis provided, including } \\
\text { elastic/plastic behavior and creep. Quantitative data drawn from [9] RTD Standard F9-5T (1974). }\end{array}$ \\
\hline Time history of primary load(s) & Steady pressure \\
\hline Time history of secondary load(s) & Various cyclic thermal \\
\hline Experimental setup, i.e. support conditions. & Documented in reports cited above. \\
\hline Measurements & deformations \\
\hline Time history of temperature field & Cyclic with thermal gradients \\
\hline $\begin{array}{l}\text { Definition of load cycles (primary and/or } \\
\text { secondary) }\end{array}$ & Cyclic thermal \\
\hline Results & Deflections compared with results of predictive methods \\
\hline Failure criterion: Total collapse, $5 \%$ strain...etc. & $\mathrm{N} / \mathrm{A}$ \\
\hline Comments & Geometry, materials and loading are fully documented in references. \\
\hline
\end{tabular}




\subsubsection{P7: Penny and Marriott - Creep of Aluminum Alloy Spherical Pressure Vessel Nozzle to Rupture}

An experimental and analytical study of creep to failure of a miniature $\mathrm{Al}$ alloy pressure vessel machined out of solid (no complications of weldments or irregular surface features) was conducted [14]. This is a controlled, simple creep experiment on an idealized pressure vessel with an integral nozzle under internal pressure using $\mathrm{Al}$ alloy as test material in order to permit the use of the elevated strain gauge technology available at the time. Deformations, local stains and times-to-rupture were all recorded. Results were used to explore the validity of the Reference stress concept to both deformations and rupture life. Records of geometry, material properties and loading are provided in sufficient detail to permit analyses to be reproduced without the need for additional input. 
Table P7 Round-Robin Benchmark Problem - CP7 "Penny and Marriott - Creep of Aluminum Alloy Pressure Vessel Nozzle to Rupture”

\begin{tabular}{|c|c|}
\hline $\begin{array}{l}\text { Case \#: P7 } \\
\text { Description }\end{array}$ & $\begin{array}{l}\text { [14] R.K. Penny and D.L. Marriott, Design for Creep, Chapman and Hall, } 2^{\text {nd }} \text { ed., 1994, Chapter } 5 . \\
\text { Additional information on material in: } \\
\text { [15] D.L. Marriott and R.K. Penny, "Strain Accumulation and Rupture During Creep under Variable Uniaxial } \\
\text { Tensile Loading”, J. Strain Analysis, Vol. 8, No. 3, 1973, pp 151- 159. } \\
\text { Additional information on pressure vessel tests in: } \\
\text { [16] R.K. Penny and D.L. Marriott, Creep of Pressure Vessels”, Paper C204/73, International Conference on } \\
\text { Creep and Fatigue in Elevated Temperature Applications, Philadelphia, September } 1973 \text { and Sheffield UK, } \\
\text { April 1974, Joint I.Mech.E/ASME publication, 1974. } \\
\text { Experimental and analytical study of creep to failure of a miniature Al alloy pressure vessel machined out of } \\
\text { solid (no complications of weldments or irregular surface features). Steady and variable pressure load to } \\
\text { rupture, several load levels. }\end{array}$ \\
\hline Issues & Steady and cyclic creep rupture, axisymmetric, reference stress \\
\hline Purpose & $\begin{array}{l}\text { To validate (then current) structural analysis methods based on finite differences, and to explore the } \\
\text { applicability of the Reference stress technique for the purpose of predicting both deformations and rupture } \\
\text { life. }\end{array}$ \\
\hline Geometry of component & $\begin{array}{l}\text { Spherical pressure vessel with integral radial nozzles, pressurized with steady or cyclic pressure. } \\
\text { Details can be found in cited reference above. }\end{array}$ \\
\hline Material type \& properties & $\begin{array}{l}\text { HK30 aircraft grade } \mathrm{Al} \text { alloy }(4 \% \mathrm{Cu} / \mathrm{Mg} / \mathrm{Si} / \mathrm{Mn}) \text { Fully worked and age hardened. } \\
\text { Elastic, time independent plastic, full creep curves at } 180^{\circ} \mathrm{C} \text {. Simplified Norton-type creep models used in } \\
\text { stress analysis. Experimental data permits more detailed modeling with either Kachanov CDM or Omega } \\
\text { models. }\end{array}$ \\
\hline Time history of primary load(s) & Steady \\
\hline Time history of secondary load(s) & N/A \\
\hline $\begin{array}{l}\text { Experimental setup, i.e. support } \\
\text { conditions. }\end{array}$ & Described in detail in citation \\
\hline Measurements & Deformations, local strains, rupture time \\
\hline Time history of temperature field & Constant uniform \\
\hline $\begin{array}{l}\text { Definition of load cycles (primary and/or } \\
\text { secondary) }\end{array}$ & Primary load cycled with specified dwell times. \\
\hline Results & Deformations, local strains, rupture time \\
\hline $\begin{array}{l}\text { Failure criterion: Total collapse, } 5 \% \\
\text { strain...etc. }\end{array}$ & Pressure boundary leakage \\
\hline Comments & Material, geometry and loading are fully documented in references \\
\hline
\end{tabular}




\subsubsection{CP8: Goodall's Experiments of Simple Plates, Plates with Notches and Cylinder/Cylinder Intersections}

A set of creep rupture experiments was performed for the purpose of validating a Goodall's and others' versions [17-20] of Calladine's approach [21] to Reference stress calculation for use in creep rupture design. This data is valuable since it includes tests on two materials, hardened aluminum alloy and 316 stainless steel. The tests included steady and cyclic primary loading. Numerical/analytical studies were conducted on materials with varying creep exponents as well as various degrees of creep ductility. 
Table CP8 Round-Robin Benchmark Problem - CP8 “Goodall's Experiments of Plain Plates, Plates with Notches and Cylinder/Cylinder Intersection”

\begin{tabular}{|c|c|}
\hline $\begin{array}{l}\text { Case \#: CP8 } \\
\text { Description }\end{array}$ & $\begin{array}{l}\text { [17] Goodall I.W., et al. “An Approximate Description of The Creep Rupture of Structure,” Int. J. Mech. } \\
\text { 1975, Vol. 17, pp 351-380. } \\
\text { [18] Goodall I.W. \& Ainsworth, R.A., "Failure of Structures by Creep,” Third International Conference on } \\
\text { Pressure Vessel Technology, April 1977, Tokyo, Japan, pp871-883. } \\
\text { The main focus of the paper was to propose and validate a creep rupture design formula. However, the } \\
\text { paper contains experimental data of creep rupture. Several Components were tested for creep rupture. } \\
\text { Components are plain plates, plates with center and edge slots, and cylinder/cylinder intersection without } \\
\text { welds. The loading was both steady and cyclic. } \\
\text { [19] Leckie, et al., ”Creep Rupture of Structures,” Proc. Royal Soc. London A, Vol. 340, pp. 323-347, } \\
\text { 1974. } \\
\text { [20] Hayhurst, D.R., et al., “The Effect of Stress Concentrations on the Creep Rupture of Tension Panels,” } \\
\text { J. Appl. Mechs., Vol 42, pp. 613-618, } 1975 \text {. }\end{array}$ \\
\hline Issues & $\begin{array}{l}\text { Creep rupture, notched components; addresses effects of limited creep ductility and different creep } \\
\text { exponents }\end{array}$ \\
\hline Purpose & To validate simplified analysis methods for creep rupture under steady and cyclic loading. \\
\hline Geometry of component & $\begin{array}{l}\text { Plain plate, plate with center hole, plate with two edge notches/slots, plate with center slot, all subjected to } \\
\text { axial in-plane loading. Cylinder/Cylinder intersection, pressurized with steady or cyclic pressure. Details } \\
\text { can be found in cited reference above. }\end{array}$ \\
\hline Material type and properties & $\begin{array}{l}\text { Plates: Precipitation hardened aluminum alloy, and Single electro-flux re-melted type } 316 \text { stainless forged } \\
\text { steel in the solution treated condition. } \\
\text { Cylinder/Cylinder intersection: } 316 \text { stainless steel. } \\
\text { Isochronous curves for Aluminum alloy are provided in paper. } \\
\text { Not clear if the rest of the material properties are available in any reference in the paper }\end{array}$ \\
\hline Time history of primary load(s) & Steady or cyclic \\
\hline Time history of secondary load(s) & N/A \\
\hline Experimental setup, i.e. support conditions. & Details in reference \\
\hline Measurements & Rupture time and deformation \\
\hline Time history of temperature field & Constant uniform \\
\hline $\begin{array}{l}\text { Definition of load cycles (primary and/or } \\
\text { secondary) }\end{array}$ & Primary load cycled with specified dwell times. \\
\hline Results & Rupture time of experiment compared with simplified reference stress method. \\
\hline Failure criterion: Total collapse, $5 \%$ strain...etc. & rupture \\
\hline Comme & \\
\hline
\end{tabular}




\subsubsection{CP9: NIL_FFS Project- Experimental and Analytical Investigation of the Elevated Temperature Performance of $a$ Number of Welded and Unwelded Pressure Vessels}

This four-part report is a detailed description of possibly the most ambitious combined experimental and theoretical program developed to study the elevated temperature deformation and failure mechanisms in welded, pressurized containments [22]. The material of construction is a variant of Grade 11. The scope of the study includes construction and testing of realistic pressure vessels, FEA to predict test performance and associated material studies to quantify creep, creep rupture and cracking behavior under both steady and cyclically varying loading. Eleven (11) vessels have been examined covering 7 different geometries and a range of thermal/mechanical loadings. Results are reported in extensive detail. The report is in Dutch. 


\section{Table CP9 Round-Robin Benchmark Problem - CP9 “NIL_FFS Project}

\begin{tabular}{|c|c|}
\hline $\begin{array}{l}\text { Case \#: CP9 } \\
\text { Description }\end{array}$ & $\begin{array}{l}\text { [22] J. van den Eikhoff and J. Prij, “N.I.L Restlevensduurproject (FFS project)”, Nederlandse Instituut } \\
\text { voor Lastechniek, Report RLD 86-01 parts A through D, December 1985. In Dutch } \\
\text { This four part report is a detailed description of possibly the most ambitious combined experimental and } \\
\text { theoretical program developed to study the elevated temperature deformation and failure mechanisms in } \\
\text { welded, pressurized containments. The material of construction is a variant of Grade } 11 \text {. The scope of the } \\
\text { study includes construction and testing of realistic pressure vessels, FEA to predict test performance and } \\
\text { associated material studies to quantify creep, creep rupture and cracking behavior under both steady and } \\
\text { cyclically varying loading. } 11 \text { vessels have been examined covering } 7 \text { different geometries and a range of } \\
\text { thermal/mechanical loadings. Results are reported in extensive detail. }\end{array}$ \\
\hline Issues & Various, all issues of concern \\
\hline Purpose & $\begin{array}{l}\text { The project is large and has multiple purposes. The purpose of interest to this Task } 9.6 \text { is that it provides an } \\
\text { unprecedented source of experimental and analytical data which can be used as a very effective baseline } \\
\text { against which to test virtually every design concept currently in the ASME Code, and being considered for } \\
\text { future use. }\end{array}$ \\
\hline Geometry of component & $\begin{array}{l}\text { Components: } \\
1 \text { and } 2 \text {. A simple cylinder with one flat end closure and the other a welded spherical cap containing an } \\
\text { in-line nozzle.; } 3 \text {. A cylinder capped by flat end closures with } 3 \text { welded on radial nozzles; } 4 \text { and } 5 . \\
\text { Capped cylinder with a single large diameter welded radial nozzle; } 6 \text {. Stepped cylinder with flat end } \\
\text { caps; } 8 \text { and 9. Plain cylinders with flat end caps; } 10 \text { and } 11 \text {. Plain cylinders with spherical end caps } \\
7 \text { and } 9 \text {. Not described. Detailed drawings provided for all parts }\end{array}$ \\
\hline Material type and properties & $\begin{array}{l}\text { 13CrMo44 (A form of Gr 11) } \\
\text { Extensive description of all material properties relevant to cyclic load analysis provided, including } \\
\text { elastic/plastic behavior and creep. All necessary data supplied in the report }\end{array}$ \\
\hline Time history of primary load(s) & Steady and cyclic pressure \\
\hline Time history of secondary load(s) & N/A \\
\hline Experimental setup, i.e. support conditions. & Pressurized with steam. Details of experimental setup provided \\
\hline Measurements & Various: deformations, time to rupture, strain...etc. \\
\hline Time history of temperature field & Various cyclic thermal histories \\
\hline $\begin{array}{l}\text { Definition of load cycles (primary and/or } \\
\text { secondary) }\end{array}$ & Cyclic Pressure \\
\hline Results & $\begin{array}{l}\text { Various experimental results on deformation and rupture. } \\
\text { FEA results. Detailed comparison of pressure vessel failures with various predictions combined with post } \\
\text { failure metallurgical examination }\end{array}$ \\
\hline Failure criterion: Total collapse, 5\% strain...etc. & $\begin{array}{l}\text { No specific failure criterion defined. The investigation was intended to identify and quantify these. } \\
\text { Deformations and cracking both described in detail, including post failure metallurgical examination of } \\
\text { microstructures and crack morphologies. }\end{array}$ \\
\hline Comments & Geometry, loading and material fully documented in cited report. \\
\hline
\end{tabular}




\subsubsection{CP10: Igari’s Ratcheting Response of Pipes and Elbows under Cyclic Displacement Controlled Loads}

Experiments were conducted on straight and elbow pipe sections under controlled transverse displacement at room and elevated temperatures - with and without internal pressure [23]. The experiments included different $\mathrm{R} / \mathrm{t}$ values as well. The material was 304 stainless steel. FEA results were compared with experiments. 
Table CP10 Round-Robin Benchmark Problem -CP10 “Igaris’ Ratcheting Response of Pipes and Elbows under Cyclic Displacement Controlled Loads"

\begin{tabular}{|c|c|}
\hline $\begin{array}{l}\text { Case \#: CP10 } \\
\text { Description }\end{array}$ & $\begin{array}{l}\text { [23] Igari t., et al., “Ratcheting Response of Pipes and Elbows Under Cyclic Displacement Controlled } \\
\text { Loads,”, Trans. } 14^{\text {th }} \text { Intl. Conference on Structural Mechanics In Reactor Technology (SMiRT 14), Lyon, } \\
\text { France, 1997. pp.117-124. }\end{array}$ \\
\hline Issues & Ratcheting \\
\hline Purpose & Validate ratcheting \\
\hline Geometry of component & Straight pipe and elbow. Full detail in reference \\
\hline Material type and properties & 304 Stainless Steel \\
\hline Time history of primary load(s) & Pressure \\
\hline Time history of secondary load(s) & Controlled cyclic displacement \\
\hline Experimental setup, i.e. support conditions & Full detail in reference \\
\hline Measurements & Stain inside and outside of pipe \\
\hline Time history of temperature field & Constant elevated \\
\hline $\begin{array}{l}\text { Definition of load cycles (primary and/or } \\
\text { secondary) }\end{array}$ & Cyclic displacement \\
\hline Results & Strain accumulation \\
\hline Failure criterion: Total collapse, $5 \%$ strain...etc. & N/A \\
\hline Comments & \\
\hline
\end{tabular}




\subsubsection{P11: Corum-Battiste Experimental and analytical data on a typical nozzle for LMFBR/CRBRP-IHX}

This work is found in two references that describe a series of creep experiments performed at ORNL, on model stainless steel pressure vessels consisting of an axisymmetrical nozzle in a spherical end cap [24, 25]. The objective was to generate information on the creep rupture of complex components. The references describe tests on three vessels of similar geometry which formed part of a larger study of 13 vessels. The vessels described here were subjected to constant and variable pressure. The primary purpose was to evaluate rupture and was not concerned with cyclically enhanced deformation by mechanisms such as ratcheting. Test results were compared with detailed predictions using an ORNL developed finite element program and material constitutive models. Details of vessel geometry, material and loading conditions are described in sufficient detail to permit the analysis to be reproduced without recourse to the use of supplementary information. 
Table P11 Round-Robin Benchmark Problem - P11 “Corum-Battiste Experimental and analytical data on a typical nozzle for LMFBR/CRBRP-IHX”

\begin{tabular}{|c|c|}
\hline $\begin{array}{l}\text { Case \#: P11 } \\
\text { Description }\end{array}$ & $\begin{array}{l}\text { [24] J.A. Clinard, R.L. Battiste, R.W. Swindeman, Y.L. Lin and R.C. Gwaltney, Elevated Temperature } \\
\text { Deformation and Failure Testing and Analysis of Nozzle-to-Spherical Shells: Specimens NS-2 and NS-1”, } \\
\text { ORNL-5939. } \\
\text { [25] J.M. Corum and R.L. Battiste, "Predictability of Long-Term Creep and Rupture in a Nozzle-to- } \\
\text { Sphere Vessel Model”, J, Pres.Ves.Tech., Vol.115, May 1993, pp122-127. } \\
\text { Creep experiments performed at ORNL, on model stainless steel pressure vessels consisting of an } \\
\text { axisymmetrical nozzle in a spherical end cap with steady and variable pressure to determine creep. This } \\
\text { work was used to validate simplified rules contained in NCC N-47 (and, subsequently, Section } \\
\text { III/Subdivision NH) for evaluation of primary pressure boundary failure by creep rupture. }\end{array}$ \\
\hline Issues & Creep, creep rupture, notch, axisymmetry \\
\hline Purpose & Validate creep strain \\
\hline Geometry of component & Spherical pressure/nozzle Fully documented in reports above \\
\hline Material type and properties & $\begin{array}{l}\text { Type } 304 \text { Stainless Steel. Extensive description of all material properties relevant to cyclic load analysis } \\
\text { provided, including elastic/plastic behavior and creep. Quantitative data drawn from [9] RTD Standard F9- } \\
\text { 5T (1974). }\end{array}$ \\
\hline Time history of primary load(s) & Steady pressure and occasional step pressure changes \\
\hline Time history of secondary load(s) & N/A \\
\hline Experimental setup, i.e. support conditions & Details provided in references. Loading provided by steam pressure. \\
\hline \multicolumn{2}{|l|}{ Measurements } \\
\hline \multicolumn{2}{|l|}{ Time history of temperature field } \\
\hline \multicolumn{2}{|l|}{$\begin{array}{l}\text { Definition of load cycles (primary and/or } \\
\text { secondary) }\end{array}$} \\
\hline Results & Comparisons of predicted rupture lives with experiment. \\
\hline Failure criterion: Total collapse, 5\% strain...etc. & Creep rupture \\
\hline Comments & \\
\hline
\end{tabular}




\subsubsection{P12: D.L. Marriott - Welded Spacer Connecting Adjacent Legs of a Serpentine Boiler Tube Platen}

This paper describes a design study of a 3-dimensional spacer in an Advanced Gas Reactor boiler platen subjected to elastic follow-up due to differential expansion of a tubing loop [26]. In addition, the complex geometry precluded factorization of linearized stresses through the tube wall into primary and secondary categories. The result was overly conservative classification of stresses as primary in the absence of clear proof that they, or any proportion of them, were in fact secondary in nature. More detailed analysis described in this paper was able to demonstrate that the primary stress was, in fact, only a small percentage of the whole, thus averting a costly and unnecessary redesign. 


\section{Table P12 Round-Robin Benchmark Problem - P12 "D.L. Marriott - Welded spacer connecting adjacent legs of a serpentine}

boiler tube platen”

\begin{tabular}{|c|c|}
\hline $\begin{array}{l}\text { Case \#: P12 } \\
\text { Description }\end{array}$ & $\begin{array}{l}\text { [26] D.L. Marriott, "Evaluation of Deformation or Load Control under Inelastic Conditions using Elastic } \\
\text { FEA”, PVP 1988, Pittsburgh, PA, June 19-23 1988, Vol. 136, pp 3-9. } \\
\text { A design study of a 3-dimensional spacer in an Advanced Gas Reactor boiler platen subjected to elastic } \\
\text { follow-up due to differential expansion of a tubing loop. In addition, the complex geometry precluded } \\
\text { factorization of linearized stresses through the tube wall into primary and secondary categories. }\end{array}$ \\
\hline Issues & Elastic followup, stress categorization \\
\hline Purpose & Validate simplified methods with elastic followup effect \\
\hline Geometry of component & $\begin{array}{l}\text { A single welded spacer connecting adjacent legs of a serpentine boiler tube platen. All relevant dimensions } \\
\text { provided }\end{array}$ \\
\hline Material type and properties & $\begin{array}{l}\text { Type } 316 \text { Stainless Steel. Design allowables from N } 47 \text { were the only properties used in the original study. } \\
\text { For future use as a benchmark problem, Omega model data from API } 579 \text { is recommended }\end{array}$ \\
\hline Time history of primary load(s) & Steady pressure \\
\hline Time history of secondary load(s) & N/A \\
\hline Experimental setup, i.e. support conditions & $\mathrm{N} / \mathrm{A}$ \\
\hline Measurements & $\mathrm{N} / \mathrm{A}$ \\
\hline Time history of temperature field & N/A \\
\hline $\begin{array}{l}\text { Definition of load cycles (primary and/or } \\
\text { secondary) }\end{array}$ & $\mathrm{N} / \mathrm{A}$ \\
\hline Results & Reclassification of stresses based on more detailed analysis \\
\hline Failure criterion: Total collapse, $5 \%$ strain...etc. & Conformance with N47 design criteria \\
\hline Comments & Scanned version of full reports available on-line or on request \\
\hline
\end{tabular}




\subsubsection{USEFUL OTHER CASES AND RESOURCES}

The literature survey led to the discovery of an additional set of problems which describe projects with potential for application as further case Round Robin candidate examples but have not been included as such in this project because they may either be incomplete, in that they fail to include all the necessary information required to reproduce the work independently, or it has not been possible to verify this fact within the timescale of this program. However, they hold promise and some may be found acceptable in that role at a later date after they have been further investigated. Some also have intrinsic interest for the information they contain, even if this is not sufficient in itself to form the basis of a Round Robin and, for that reason they have been listed for the benefit of interested readers of this report.

\subsubsection{Use of Isochronous curves to predict inelastic strain:}

Recent work by Koves and Zhao [27-29] focused on the use of isochronous curves under primary and secondary loading and multiaxial state of stress. Their work can be found in the following references. Also, Abou-Hanna and McGreevy conducted a similar study [30].

\subsubsection{Shakedown, Ratchet and Reversed Plasticity Limits}

Alan Ponter has been developing simplified automated finite element based techniques to determine shakedown, ratchet and reversed plasticity limits as well as inelastic strain under cyclic loading for over a decade. Ponter has many publications on the subject. These publications contain valuable data that can be used to verify other simplified methods for predicting cyclic inelastic strain accumulation as well as ratchet and shakedown limits. His approach is called the Linear Matching Method, and was discussed in Subtask 9.4. Some of his publications were cited in Subtask 9.4; some references are cited here as well [31-34]. His approach was verified against full inelastic analysis. The components he analyzed ranged from plates with holes, circular plates simply supported, tubes with thermal gradients, and flat plates with tension and thermal gradients.

Subtask 9.4 refers to a new simplified method, developed by McGreevy and AbouHanna, for identifying ratchet and shakedown limits as well as predicting inelastic core strain based on definition of component elastic core [35]. Their work was verified using several components, mainly those that Ponter had published as well as a tube under thermal shock/thermal travel wave. Their work is cited and discussed briefly in subtask 9.4 report. 


\subsubsection{Inelastic Strain in Variable Cycle Loading at Elevated Temperatures:}

McGreevy and Abou-Hanna compared the inelastic strains of full inelastic FEA analysis to those predicted by the NH Simplified approach for a Bree type tube [36]. Their results show the extent of accuracy of the B-1 Test of ASME Appendix T, and the extent of conservatism when load cycles are combined into block loading. The results may be useful for comparison with other simplified analysis techniques, especially if such methods are used in conjunction with existing approaches in ASME NH Appendix T, e.g. grouping of cycles into blocks. The loading history considered variable thermal cyclic loading by a constant thermal gradient across tube wall and a constant primary pressure loading. The pressure did change from cycle to another.

\subsubsection{The ECCC Database of Component Tests and Assessments:}

The ECCC reports include information on 36 high temperature components tests, involving ferritic and austenitic steels, with an indication of the assessment route(s) performed where appropriate [37,38]. The purpose of the ECCC review of existing component testing experience was to ascertain the availability of existing case study material which could be first evaluated before embarking on a costly test program. The tables summarizing these cases have been extracted from the ECCC report and presented in Appendix A. The tables are provided in this report, as they may be helpful additional information for the establishment of round-robin benchmark analysis and/or experiments.

\subsubsection{MINIMUM MATERIAL DATA REQUIREMENTS}

As an outcome of the work conducted in the first 5 subtasks of this project, a number of established and novel design methods of possible use in updating ASME ETD Code have been collected and reviewed. A number of new and established procedures stood out as being worthy of further consideration. These were:

1. The Reference Stress concept which has long been an accepted method for primary design assessment as part of R5

2. The cyclic Reference stress for shakedown and ratcheting analysis

3. One version of the cyclic Reference stress known as the Linear Matching Method (due to be adopted by R5), and

4. The Hybrid Method, which is a development of the Bree type analyses currently offered in Appendix $\mathrm{T}$ of $\mathrm{NH}$, based on the elastic core concept.

A significant feature of these candidates and one that probably played a major part in their being short listed in this way, is that their minimum material data requirements need not exceed what is available currently in $\mathrm{NH}$. In fact, the Reference Stress concept actually requires less than existing design procedures in order to implement the component analysis. The data required to compare design performance against code design criteria is a variable at present, since it depends on how precisely it is decided to 
implement this methodology, assuming it is accepted in due course as an ASME Code procedure. At minimum, a conservative version can be implemented which utilizes no more in the way of material data than the current $\mathrm{NH}$ procedures. With the addition of a further material parameter, the creep ductility $\lambda$, it might be possible to develop a less conservative Reference Stress procedure.

Table 9.6.7.1 below provides a list of material data that are required for the simplified methods suggested for primary/steady load analysis. These methods address either creep rupture or creep deformation. Note, the Linear Matching Method and Hybrid Approach are based upon limit load solutions, and hence are identical to the Reference Stress approach - the authors elected to explicitly include these in the listing as they are both included in cyclic analysis approaches.

Table 9.6.7.1 Minimum material data requirement for primary load analysis, (deformation and/or rupture)

\begin{tabular}{|c|c|c|c|}
\hline Data & $\begin{array}{c}\text { NH } \\
\text { Simplified } \\
\text { Method }\end{array}$ & $\begin{array}{c}\text { Reference } \\
\text { Stress }\end{array}$ & $\begin{array}{c}\text { Linear Matching } \\
\text { Method \& Hybrid } \\
\text { Approach }\end{array}$ \\
\hline $\begin{array}{c}\text { Elastic Mod. \& Poisson's } \\
\text { Ratio }\end{array}$ & $\mathrm{X}$ & $\mathrm{X}$ & $\mathrm{X}$ \\
\hline $\begin{array}{c}\text { Yield Strength }\left(\mathrm{S}_{\mathrm{y}}\right) \\
\begin{array}{c}\text { Creep data/Isochronous } \\
\text { curves or creep rate } \\
\text { parameters }\end{array}\end{array}$ & $\mathrm{X}$ & $\mathrm{X}$ & $\mathrm{X}$ \\
\hline Creep Rupture Data & $\mathrm{X}$ & $\mathrm{X}$ & $\mathrm{X}$ \\
\hline Thermal conductivity & $\mathrm{X}$ & $\mathrm{X}$ & $\mathrm{X}$ \\
\hline Creep Ductility $(\lambda)$ & & optional & optional \\
\hline
\end{tabular}

Table 9.6.7.2 describes the minimum data required for cyclic analysis. The cyclic analysis includes determination of the shakedown, ratchet, or reversed plasticity limits for deformation or strain. It should be noted that the Linear Matching Method (LMM) uses a cyclic reference stress approach in cyclic analysis. The Hybrid Method utilizes an elastic core approach. It is recommended that both the best estimate and the design data be provided to be able to assess the extent of conservatism inherent in the safety factors implemented in the stress allowables. 
Table 9.6.7.2 Minimum material data requirement for cyclic load analysis

\begin{tabular}{|c|c|c|c|}
\hline Data & $\begin{array}{c}\text { NH } \\
\text { Simplified } \\
\text { Method }\end{array}$ & $\begin{array}{c}\text { Linear Matching } \\
\text { Method/Reference } \\
\text { Stress }\end{array}$ & $\begin{array}{c}\text { Hybrid } \\
\text { Method }\end{array}$ \\
\hline $\begin{array}{c}\text { Elastic Mod. \& } \\
\text { Poisson's Ratio }\end{array}$ & $\mathrm{X}$ & $\mathrm{X}$ & $\mathrm{X}$ \\
\hline Yield Strength (S) & $\mathrm{X}$ & $\mathrm{X}$ & $\mathrm{X}$ \\
\hline $\begin{array}{c}\text { Creep } \\
\text { data/Isochronous } \\
\text { curves or creep rate } \\
\text { parameters }\end{array}$ & $\mathrm{X}$ & $\mathrm{X}$ & $\mathrm{X}$ \\
\hline $\begin{array}{c}\text { Creep Rupture Data } \\
\text { Thermal conductivity }\end{array}$ & $\mathrm{X}$ & $\begin{array}{c}\text { Optional - may use in } \\
\text { one application of } \\
\text { this approach }\end{array}$ & $\mathrm{X}$ \\
\hline Creep Ductility $(\lambda)$ & & $\mathrm{X}$ & $\mathrm{X}$ \\
\hline
\end{tabular}

**The Hybrid Method is capable of providing the cyclic reference stress (shakedown or ratcheting) that the Linear Matching Method provides. Implementing creep ductility effects on cyclic creep rupture could readily be applied as well.

\subsubsection{SUMMARY}

The objective of subtask 9.6 was to recommend a set of round-robin structural problems for comparison and assessment of simplified analysis methods and identify minimum material data requirement for such analysis. A survey of government laboratories and industry was conducted, with limited response, which led to several cases of interest. A literature survey led to many interesting cases, most of which included experimental results for steady and cyclic load conditions at elevated temperatures. These cases were summarized; references contain details that should enable independent reconstruction of the experiments and/or the models.

The summary of the cases also highlighted a list of issues the authors felt needed to be addressed in the round-robin, such as elastic follow-up and creep ductility, or issues upon which the experiment or analysis already focused.

The report also includes sufficient documentation of several other studies and references that contain information deemed useful in preparing for round-robin verification problems or for documenting for possible future use by ASME, NRC, DOE, and researchers in the ETD community. The report also included a detailed survey prepared by the European Commission ECCC of experiments conducted in the past few decades. 
The report highlighted a set of minimum material data required for implementing various simplified analysis methods. The authors recommended a Task Force be formed to proceed with implementing a round-robin, whereby the list of simplified methods may be expanded upon if desired by the Task Force.

The report may also be helpful to other Gen IV Tasks, SG-ETD Task Forces, or similar whose focus is on creep-fatigue, crack growth and weld studies since these issues are treated in some of the cases presented. Some of the simplified methods likely provide relevant output required as input for assessment to these other failure modes or issues. 


\section{REFERENCES:}

1. Kamal, S.A., Chern, J.M. and, Pai, D.H., “Applications of one-Dimensional Models in Analysis,” ASME Paper 80-C2/NE-17, presented at Century-2 Pressure Vessel and Piping Conference, San Francisco, CA, Aug. 1980.

2. Recommended Practices in ETD: A compendium of Breeder Reactor Experiences (1970-1987), Vol. II - Preliminary Design and Simplified Methods, Ch. 6.0 Simplified Methods by I.W. Goodall, A.K. Dhalla and T. Nagata, WRC Bulletin 363 - May 1991.

3. J. du Plessis et al, “Core Outlet Hot Gas Duct V-Ring Analysis Report”, PBMR, Document Number T100536, April, 2009

4. Bell, R.T. "Interim Results from CTS/Harwell Ratcheting Experiments on Thin Cylinders,” ND-M-352(R) Risley Nuclear Power Development, April 1978.

5. T. Igari, "Mechanism-Based Evaluation of Thermal Ratcheting due to Traveling Temperature Distribution,” Trans. ASME, Vol. 122, May 2000, pp. 130-138.

6. D.S. Griffin, A.K. Dhalla, W.S. Woodward, "Validation of Inelastic Analysis by Full-Scale Component Testing”, 42/Vol. 109, Feb 1987.

7. W.S. Woodward and A.K. Dhalla, "Evaluation of Inelastic Strain Accumulation for the FFTF/IHX Nozzle Proof Test,” Westinghouse Electric Corporation, Advanced Reactors division, Report \#WARD-HT-94000-10 prepared for USDOE under Contract \#DE-AMO2-76CH94000, September 1980.

8. W.K. Sartory, Analytical Investigation of the Applicability of Simplified Ratcheting and Creep-Fatigue Rules to LMFBR Component Geometries - Two Dimensional Axisymmetrical Structures. ORNL/TM-5616, December 1976.

9. RDT Standard F9-5T, Guidelines arid Procedures for Design of Nuclear System Components at Elevated Temperature, U.S. Atomic Energy Commission (September 1974).

10. P.J. Budden, "Analysis of the Type IV failures of three welded ferritic pressure vessels”, Elsevier, Int’l Journal of PVP, Vol. 75, Issue 6, 1998, pp 509-519.

11. W.K. Sartory, Analytical Investigation of the Applicability of Simplified Ratcheting and Creep-Fatigue Rules to LMFBR Component Geometries - Two Dimensional Axisymmetrical Structures. ORNL/TM-5616, December 1976.

12. W. K. Sartory and R.L. Battiste, "Inelastic Ratcheting Analysis of the 21/4 Cr 1Mo Steel Stepped-wall Region of Specimen TTT-3, ORNL-5626, April 1980. 
13. R.L. Battiste and M. Richardson, "Elevated Temperature Benchmark Tests of 21/4Cr 1Mo Steel Simply supported Beams and a Circular Plate subjected to TimeVarying Loadings, ORNL/TM-7085, May 1980.

14. R.K. Penny and D.L. Marriott, Design for Creep, Chapman and Hall, $2^{\text {nd }}$ ed., 1994.

15. D.L. Marriott and R.K. Penny, "Strain Accumulation and Rupture During Creep under Variable Uniaxial Tensile Loading”, J. Strain Analysis, Vol. 8, No. 3, 1973, pp 151- 159.

16. R.K. Penny and D.L. Marriott, Creep of Pressure Vessels”, Paper C204/73, International Conference on Creep and Fatigue in Elevated Temperature Applications, Philadelphia, September 1973 and Sheffield UK, April 1974, Joint I.Mech.E/ASME publication, 1974.

17. Goodall I.W., et al. "An Approximate Description of The Creep Rupture of Structure,” Int. J. Mech. 1975, Vol. 17, pp 351-380.

18. Goodall I.W. \& Ainsworth, R.A., "Failure of Structures by Creep,” Third International Conference on Pressure Vessel Technology, April 1977, Tokyo, Japan, pp871-883.

19. Leckie, et al., "Creep Rupture of Structures,” Proc. Royal Soc. London A, Vol. 340, pp. 323-347, 1974.

20. Hayhurst, D.R., et al., "The Effect of Stress Concentrations on the Creep Rupture of Tension Panels,” J. Appl. Mechs., Vol 42, pp. 613-618, 1975.

21. Calladine, C.R., A Rapid Method for Estimating the Greatest Stress in a Structure Subjected to Creep, Proc. I. Mech. E., 178, (1963-1964).

22. J. van den Eikhoff and J. Prij, "N.I.L Restlevensduurproject (FFS project)", Nederlandse Instituut voor Lastechniek, Report RLD 86-01 parts A through D, December 1985. In Dutch.

23. Igari t., et al., "Ratcheting Response of Pipes and Elbows Under Cyclic Displacement Controlled Loads,”, Trans. $14^{\text {th }}$ Intl. Conference on Structural Mechanics In Reactor Technology (SMiRT 14), Lyon, France, 1997. pp.117-124.

24. J.A. Clinard, R.L. Battiste, R.W. Swindeman, Y.L. Lin and R.C. Gwaltney, Elevated Temperature Deformation and Failure Testing and Analysis of Nozzleto-Spherical Shells: Specimens NS-2 and NS-1", ORNL-5939.

25. J.M. Corum and R.L. Battiste, "Predictability of Long-Term Creep and Rupture in a Nozzle-to-Sphere Vessel Model”, J, Pres.Ves.Tech., Vol.115, May 1993, pp122-127. 
26. D.L. Marriott, "Evaluation of Deformation or Load Control under Inelastic Conditions using Elastic FEA”, PVP 1988, Pittsburgh, PA, June 19-23 1988, Vol. 136, pp 3-9.

27. M. Zhao and W. Koves, ”Applications of Isochronous Data in Creep Analysis,” Proceedings of PVP2007-26095, July2007, San Antonio, Texas.

28. W. Koves and M. Zhao, "Comparison of the Isochronous Method and A TimeExplicit Model for Creep Analysis,” Proceedings of PVP2008-61579, July 2008, Chicago, Illinois.

29. M. Zhao and W. Koves, "The Isochronous Stress-Strain Method With MultiAxial Stress States And Variable Loading Conditions,” Proceedings of PVP200977090, July 2009, Prague, Czech Republic.

30. J. Abou-Hanna and Timothy McGreevy,” Simplified Inelastic Time Independent (SITI) Method for Predicting Creep,” Proceedings of PVP2007-26060, July2007, San Antonio, Texas.

31. R. S. Ponter and M. Englehardt, "Shakedown limits for a general yield condition: implementation and application for a Von Mises yield condition," Eur. J. Mech. A/Solids 19, 423 (2000).

32. Seshadri, R., Fernando, C.P.D., 1992, "Limit Loads of Mechanical Components and Structures using the GLOSS R-Node Method,” Journal of Pressure Vessel Technology, Vol. 114, pp. 201-208.

33. A.R.S. Ponter and H. Chen, A minimum theorem for cyclic load in excess of shakedown, with application to the evaluation of a ratchet limit, Eur. J. Mech. A/Solids 20 (2001) pp 539-553.

34. A.R.S. Ponter and H. Chen, A method for the evaluation of a ratchet limit and the amplitude of plastic strain for bodies subjected to cyclic loading, Eur. J. Mech. A/Solids 20 (2001) pp 555-571.

35. T. E. McGreevy, and J. Abou-Hanna, A Hybrid Approach to Simplified Methods, October 27, 2008; to be published.

36. T.E. McGreevy and J. Abou-Hanna, “Applicability Of Simplified Methods To Alloy 617 In Excess Of 649 C,” Proceedings of PVP2007-26672, July2007, San Antonio, Texas.

37. ECCC Recommendations Volume 3, 2005, 'Part V: Testing practices for multiaxial features and components', eds. Brown, T.B. \& Holdsworth, S.R.

38. Holdsworth, S.R., 2002, 'Overview of activities of the Structural Mechanics Cluster of the Plant Life Assessment Network', Materials at High Temperature, 19(2), 69-74. 


\section{APPENDIX A}

ECCC List of Components Tests [37]

ECCC RECOMMENDATIONS - VOLUME 9 Part III [Issue 1]

HIGH TEMPERATURE COMPONENT ANALYSIS

DATABASE OF COMPONENT TESTS AND

ASSESSMENTS 


\begin{tabular}{|c|c|c|c|c|c|c|c|c|c|c|}
\hline No & TYPE & $\begin{array}{c}\text { TEST } \\
\text { DESCRIPTION }\end{array}$ & MATERIAL & $\begin{array}{l}\text { STARTER } \\
\text { CRACK(S) }\end{array}$ & TEMP & LOADING & DURATION & $\begin{array}{l}\text { MONITORING } \\
\text { INFORMATION }\end{array}$ & REFERENCES & $\begin{array}{l}\text { ANALYSIS } \\
\text { ROUTE }\end{array}$ \\
\hline 1 & Ferritic & $\begin{array}{l}\text { MEL Vessel FM1 } \\
\text { Ri }=115 \mathrm{~mm} \\
\mathrm{t}=80 \mathrm{~mm}\end{array}$ & $\begin{array}{l}0.5 \mathrm{CrMoV} \\
\text { parent }\end{array}$ & $\begin{array}{l}\text { Various circ. } \\
\text { Notches }\end{array}$ & $565^{\circ} \mathrm{C}$ & $\mathrm{P}=62.5 \mathrm{MPa}$ & $\begin{array}{l}\text { 1.6kh (total failure at } \\
\text { site of deepest fully } \\
\text { circ.notch) }\end{array}$ & $\begin{array}{l}\text { Creep strain } \\
\text { Strain gauges } \\
\text { Notch CMOD } \\
\text { Crack size } \\
\text { DCPD } \\
\text { Ultrasonics } \\
\text { Post test exam. }\end{array}$ & $\begin{array}{l}\text { (a) Coleman, Price and } \\
\text { Williams, ICF4, 649-682, } \\
1977 . \\
\text { (b) Ainsworth \& Coleman, } \\
\text { Fat. Fract. Eng. Mater. } \\
\text { Struct., 10, 129-140, } 1987 .\end{array}$ & R5 \\
\hline 2 & Ferritic & $\begin{array}{l}\text { MEL Vessel FM2 } \\
\text { Ri }=115 \mathrm{~mm} \\
\mathrm{t}=60 \mathrm{~mm}\end{array}$ & $\begin{array}{l}0.5 \mathrm{CrMoV} \\
\text { parent } \\
2.25 \mathrm{Cr} 1 \mathrm{Mo} \\
\text { welds (AW \& } \\
\text { SR) }\end{array}$ & $\begin{array}{l}\text { Various } \\
\text { defects spark } \\
\text { machined in } \\
\text { circ. welds } \\
\text { (HAZ \& Type } \\
\text { IV zones). }\end{array}$ & $585^{\circ} \mathrm{C}$ & $\mathrm{P}=35 \mathrm{MPa}$ & $\begin{array}{l}\text { 46kh } \\
\text { (steam leak) }\end{array}$ & $\begin{array}{l}\text { Creep strain } \\
\text { Creep pips } \\
\text { Strain gauges } \\
\text { Crack size } \\
\text { Ultrasonics } \\
\text { Post test exam. }\end{array}$ & $\begin{array}{l}\text { Jones \& Coleman, } \\
\text { (a) RD/B/6242/R89 } \\
\text { (b) CFEMS4, 605-619, } \\
1990 . \\
\text { Budden } \\
\text { TIGM/REP/0088/93 }\end{array}$ & R5 \\
\hline 3 & Ferritic & $\begin{array}{l}\text { MEL Vessel } \\
\text { FM2A } \\
\text { Ri }=115 \mathrm{~mm} \\
t=60 \mathrm{~mm}\end{array}$ & $\begin{array}{l}0.5 \mathrm{CrMoV} \\
\text { parent } \\
2.25 \mathrm{Cr} 1 \mathrm{Mo} \\
\text { welds (AW \& } \\
\text { SR) }\end{array}$ & $\begin{array}{l}\text { Various } \\
\text { defects spark } \\
\text { machined in } \\
\text { circ. welds } \\
\text { (HAZ \& Type } \\
\text { IV zones). }\end{array}$ & $585^{\circ} \mathrm{C}$ & $\mathrm{P}=35 \mathrm{MPa}$ & $\begin{array}{l}\text { 54kh } \\
\text { (steam leak) }\end{array}$ & $\begin{array}{l}\text { Creep strain } \\
\text { Creep pips } \\
\text { Strain gauges } \\
\text { Crack size } \\
\text { Ultrasonics } \\
\text { Post test exam. }\end{array}$ & $\begin{array}{l}\text { Budden } \\
\text { (a) TIGM/REP/0089/83 } \\
\text { (b) Int. J. Pres. Vess. \& } \\
\text { Piping. 75, 509-519 1998. }\end{array}$ & R5 \\
\hline
\end{tabular}




\begin{tabular}{|c|c|c|c|c|c|c|c|c|c|c|}
\hline No & TYPE & $\begin{array}{c}\text { TEST } \\
\text { DESCRIPTION }\end{array}$ & MATERIAL & $\begin{array}{l}\text { STARTER } \\
\text { CRACK(S) }\end{array}$ & TEMP & LOADING & DURATION & $\begin{array}{l}\text { MONITORING } \\
\text { INFORMATION }\end{array}$ & REFERENCES & $\begin{array}{l}\text { ANALYSIS } \\
\text { ROUTE }\end{array}$ \\
\hline & Ferritic & $\begin{array}{l}\text { MEL Vessel } \\
\text { TWMC1A } \\
\text { Ri }=212 \mathrm{~mm} \\
\mathrm{t}=28 \mathrm{~mm}\end{array}$ & $\begin{array}{l}0.5 \mathrm{CrMoV} \\
\text { parent } \\
2.25 \mathrm{Cr} 1 \mathrm{Mo} \\
\text { welds (AW \& } \\
\text { SR) }\end{array}$ & None & $575^{\circ} \mathrm{C}$ & $\mathrm{P}=7 \mathrm{MPa}$ & $\begin{array}{l}47 \mathrm{kh} \\
\text { (steam leak due to } \\
\text { Type IV crack in end } \\
\text { cap weld) }\end{array}$ & $\begin{array}{l}\text { Creep strain } \\
\text { Creep pips } \\
\text { Strain gauges } \\
\text { Crack size } \\
\text { Ultrasonics } \\
\text { Post test exam. }\end{array}$ & $\begin{array}{l}\text { Budden } \\
\text { (a) EPD/GEN/REP/0297/98 } \\
\text { (b) Int. J. Pres. Vess. \& } \\
\text { Piping. 75, 509-519 } 1998 .\end{array}$ & R5 \\
\hline 5 & Ferritic & $\begin{array}{l}\text { MEL Vessel } \\
\text { TWMC2 } \\
\text { Ri }=230 \mathrm{~mm} \\
\mathrm{t}=28 \mathrm{~mm}\end{array}$ & $\begin{array}{l}0.5 \mathrm{CrMoV} \\
\text { parent } \\
2.25 \mathrm{Cr} 1 \mathrm{Mo} \\
\text { welds (AW \& } \\
\text { SR) }\end{array}$ & None & $575^{\circ} \mathrm{C}$ & $\mathrm{P}=7 \mathrm{MPa}$ & $\begin{array}{l}59 \mathrm{kh} \\
\text { (steam leak due to } \\
\text { Type IV crack in end } \\
\text { cap weld) }\end{array}$ & $\begin{array}{l}\text { Creep strain } \\
\text { Creep pips } \\
\text { Strain gauges } \\
\text { Crack size } \\
\text { Ultrasonics } \\
\text { Post test exam. }\end{array}$ & $\begin{array}{l}\text { Budden } \\
\text { (a) EPD/GEN/REP/0297/98 } \\
\text { (b) Int. J. Pres. Vess. \& } \\
\text { Piping. 75, 509-519 } 1998 .\end{array}$ & R5 \\
\hline & Ferritic & $\begin{array}{l}\text { Large Bore } \\
\text { Branch } \\
R i=229 \mathrm{~mm} \\
T=32 \mathrm{~mm} \\
r i=146 \mathrm{~mm} \\
t=16 \mathrm{~mm}\end{array}$ & $\begin{array}{l}0.5 \mathrm{CrMoV} \\
\text { parent } \\
2.25 \mathrm{Cr} 1 \mathrm{Mo} \\
\text { weld (SR) }\end{array}$ & None & $\begin{array}{l}585^{\circ} \mathrm{C} \\
\left(535^{\circ} \mathrm{C} \text { in }\right. \\
\text { service) }\end{array}$ & $\begin{array}{l}\mathrm{P}=3.6 \mathrm{MPa} \\
\text { Out-of-plane moment }= \\
30 \mathrm{kNm}\end{array}$ & $\begin{array}{l}11.7 \mathrm{kh} \\
\text { (stopped - NDT } \\
\text { indication of deep } \\
\text { Type IV crack at } \\
\text { tensile flank) }\end{array}$ & $\begin{array}{l}\text { Creep strain } \\
\text { Creep pips } \\
\text { Strain gauges } \\
\text { Crack size } \\
\text { Ultrasonics } \\
\text { Post test exam. }\end{array}$ & $\begin{array}{l}\text { AI Laham } \\
\text { EPD/GEN/REP/0371/98 } \\
\text { P J Budden, Creep Crack } \\
\text { Growth Assessment of the } \\
\text { Large Bore Branch Vessel } \\
\text { Test, E/EAN/GEN/0023/00. } \\
2000 \text {. } \\
\text { R D Patel, S Al Laham and } \\
\text { P J Budden, R5 Creep } \\
\text { Assessment of Welded } \\
\text { Trunnion \& Large Bore } \\
\text { Branch Components, } \\
\text { CFEMS Ap }\end{array}$ & R5 \\
\hline
\end{tabular}




\begin{tabular}{|c|c|c|c|c|c|c|c|c|c|c|}
\hline No & TYPE & $\begin{array}{c}\text { TEST } \\
\text { DESCRIPTION }\end{array}$ & MATERIAL & $\begin{array}{l}\text { STARTER } \\
\text { CRACK(S) }\end{array}$ & TEMP & LOADING & DURATION & $\begin{array}{l}\text { MONITORING } \\
\text { INFORMATION }\end{array}$ & REFERENCES & $\begin{array}{l}\text { ANALYSIS } \\
\text { ROUTE }\end{array}$ \\
\hline 7 & Ferritic & $\begin{array}{l}\text { Medium Bore } \\
\text { Branch } \\
\mathrm{Ri}=210 \mathrm{~mm} \\
\mathrm{~T}=18 \mathrm{~mm} \\
\mathrm{ri}=56 \mathrm{~mm} \\
\mathrm{t}=8 \mathrm{~mm}\end{array}$ & $\begin{array}{l}0.5 \mathrm{CrMoV} \\
\text { parent } \\
2.25 \mathrm{Cr} 1 \mathrm{Mo} \\
\text { weld (SR) }\end{array}$ & None & $590^{\circ} \mathrm{C}$ & $\mathrm{P}=4.0 \mathrm{MPa}$ & $\begin{array}{l}20.038 \mathrm{kh} \\
\text { (completed, } \\
\text { transverse through- } \\
\text { wall crotch crack) }\end{array}$ & $\begin{array}{l}\text { Creep strain } \\
\text { Creep pips } \\
\text { Strain gauges } \\
\text { Crack size } \\
\text { Ultrasonics } \\
\text { Post test exam. }\end{array}$ & $\begin{array}{l}\text { R D Patel, R5 Medium Bore } \\
\text { Branch Life Assessment, } \\
\text { BEGL Report } \\
\text { E/REP/ATEC/0005/GEN/01 } \\
\text { Post test examination } \\
\text { results: } \\
\text { E/EAN/MATS/0024/AGR/01 }\end{array}$ & R5 \\
\hline 8 & Ferritic & \begin{tabular}{|l} 
Pipework \\
Trunnion \\
Ri $=232 \mathrm{~mm}$ \\
$T=26 \mathrm{~mm}$ \\
ri $=149 \mathrm{~mm}$ \\
$\mathrm{t}=16 \mathrm{~mm}$
\end{tabular} & $\begin{array}{l}0.5 \mathrm{CrMoV} \\
\text { parent } \\
2.25 \mathrm{Cr} 1 \mathrm{Mo} \\
\text { weld (SR) } \\
\text { Ex-service } \\
(91.7 \mathrm{kh})\end{array}$ & None & $\begin{array}{l}585^{\circ} \mathrm{C} \\
\left(527^{\circ} \mathrm{C} \text { in }\right. \\
\text { service })\end{array}$ & $\begin{array}{l}\mathrm{P}=3.5 \mathrm{MPa} \\
\text { In-plane moment }=11 \mathrm{kNm}\end{array}$ & $\begin{array}{l}15.4 \mathrm{kh} \\
\text { (stopped) }\end{array}$ & $\begin{array}{l}\text { Creep strain } \\
\text { Creep pips } \\
\text { Strain gauges } \\
\text { Crack size } \\
\text { Ultrasonics } \\
\text { Post test exam. }\end{array}$ & $\begin{array}{l}\text { R D Patel, R5 Trunnion Life } \\
\text { Assessment, BEGL Report } \\
\text { E/REP/GEN/0036/00, } \\
\text { October 2000. } \\
\text { R D Patel, S Al Laham and } \\
\text { P J Budden, R5 Creep } \\
\text { Assessment of Welded } \\
\text { Trunnion \& Large Bore } \\
\text { Branch Components, } \\
\text { CFEMS April } 2001\end{array}$ & R5 \\
\hline 9 & Austenitic & $\begin{array}{l}\text { BRITE } 2147 \\
\text { Vessel } 1 \\
\text { Ri }=75 \mathrm{~mm} \\
\mathrm{t}=40 \mathrm{~mm}\end{array}$ & $\begin{array}{l}316 \mathrm{~L}(\mathrm{~N}) \\
\text { parent } \\
\text { and welds } \\
\text { (AW \& SR) }\end{array}$ & None & $\begin{array}{l}510^{\circ} \mathrm{C}- \\
620^{\circ} \mathrm{C}\end{array}$ & $P=63 \mathrm{MPa}$ & $\begin{array}{l}14.7 \mathrm{kh} \\
\text { (stopped) }\end{array}$ & $\begin{array}{l}\text { Creep strain } \\
\text { Creep pips } \\
\text { Strain gauges } \\
\text { Crack size } \\
\text { Ultrasonics }\end{array}$ & $\begin{array}{l}\text { Budden } \\
\text { TIGM/REP/0069/93 } \\
\text { O'Donnell } \\
\text { EPD/GEN/REP/0287/98 }\end{array}$ & R5 \\
\hline
\end{tabular}




\begin{tabular}{|c|c|c|c|c|c|c|c|c|c|c|}
\hline No & TYPE & $\begin{array}{c}\text { TEST } \\
\text { DESCRIPTION }\end{array}$ & MATERIAL & $\begin{array}{l}\text { STARTER } \\
\text { CRACK(S) }\end{array}$ & TEMP & LOADING & DURATION & $\begin{array}{l}\text { MONITORING } \\
\text { INFORMATION }\end{array}$ & REFERENCES & $\begin{array}{l}\text { ANALYSIS } \\
\text { ROUTE }\end{array}$ \\
\hline 10 & Austenitic & $\begin{array}{l}\text { BRITE } 2147 \\
\text { Vessel } 2 \\
\text { Ri }=75 \mathrm{~mm} \\
\mathrm{t}=40 \mathrm{~mm}\end{array}$ & $\begin{array}{l}316 \mathrm{~L}(\mathrm{~N}) \\
\text { parent } \\
\text { and welds } \\
\text { (AW \& SR) }\end{array}$ & $\begin{array}{l}\text { Various } \\
\text { defects spark } \\
\text { machined in } \\
\text { centre line of } \\
\text { circ. welds }\end{array}$ & $\begin{array}{l}510^{\circ} \mathrm{C}- \\
620^{\circ} \mathrm{C}\end{array}$ & $\mathrm{P}=63 \mathrm{MPa}$ & $\begin{array}{l}11.5 \mathrm{kh} \\
\text { (stopped - increase } \\
\text { in hoop strain at SR } \\
\text { weld but NDT did not } \\
\text { reveal any cracking) }\end{array}$ & $\begin{array}{l}\text { Creep strain } \\
\text { Creep pips } \\
\text { Strain gauges } \\
\text { Crack size } \\
\text { Ultrasonics }\end{array}$ & $\begin{array}{l}\text { Budden } \\
\text { TIGM/REP/DO69/93 } \\
\text { O'Donnell } \\
\text { (a) EPD/GEN/REP/0287/98 } \\
\text { (b) EPD/GEN/REP/0319/98 }\end{array}$ & R5 \\
\hline 11 & 1 Austenitic & $\begin{array}{l}\text { HYA Superheater } \\
\text { Header (1C1/4) } \\
\text { Lead Joint } \\
\mathrm{Ri}=152.4 \mathrm{~mm} \\
\mathrm{~T}=63.5 \mathrm{~mm} \\
\mathrm{ri}=51.6 \mathrm{~mm} \\
\mathrm{t}=62.7 \mathrm{~mm} \\
\text { (tapered) }\end{array}$ & $\begin{array}{l}318 \mathrm{H} \text { parent } \\
\text { and weld } \\
\text { (AW) } \\
\text { Ex-service }\end{array}$ & $\begin{array}{l}\text { Reheat crack } \\
\text { Max depth } \\
20 \mathrm{~mm}\end{array}$ & $550^{\circ} \mathrm{C}$ & $\begin{array}{l}\mathrm{P}=18.8 \mathrm{MPa} \\
\text { Nozzle end load }=15.4 \mathrm{kN} \\
\text { Thermal Shocks }\left(510^{\circ} \mathrm{C}-\right. \\
\left.330^{\circ} \mathrm{C} \text { in } 1 \mathrm{~h}\right) \text { every } 500 \mathrm{~h}\end{array}$ & $\begin{array}{l}8.8 \mathrm{kh} \\
+17 \text { thermal shocks } \\
\text { (stopped) }\end{array}$ & $\begin{array}{l}\text { Creep strain } \\
\text { Strain gauges } \\
\text { Crack size } \\
\text { Ultrasonics } \\
\text { Post test exam. }\end{array}$ & & - \\
\hline 12 & 2 Austenitic & $\begin{array}{l}\text { HYA Superheater } \\
\text { Header }(2 \mathrm{~A} 1 / 1) \\
\text { Pressure Only } \\
\mathrm{Ri}=152.4 \mathrm{~mm} \\
\mathrm{~T}=63.5 \mathrm{~mm} \\
\mathrm{ri}=51.6 \mathrm{~mm} \\
\mathrm{t}=62.7 \mathrm{~mm} \\
\text { (tapered) }\end{array}$ & $\begin{array}{l}316 \mathrm{H} \text { parent } \\
\text { and weld } \\
\text { (AW) } \\
\text { Ex-service }\end{array}$ & Reheat crack & $550^{\circ} \mathrm{C}$ & $\mathrm{P}=18.8 \mathrm{MPa}$ & $20 \mathrm{kh}$ (planned) & $\begin{array}{l}\text { Creep strain } \\
\text { Strain gauges } \\
\text { Crack size } \\
\text { Ultrasonics } \\
\text { Post test exam. }\end{array}$ & & - \\
\hline
\end{tabular}




\begin{tabular}{|c|c|c|c|c|c|c|c|c|c|c|}
\hline No & TYPE & $\begin{array}{c}\text { TEST } \\
\text { DESCRIPTION }\end{array}$ & MATERIAL & $\begin{array}{l}\text { STARTER } \\
\text { CRACK(S) }\end{array}$ & TEMP & LOADING & DURATION & $\begin{array}{l}\text { MONITORING } \\
\text { INFORMATION }\end{array}$ & REFERENCES & $\begin{array}{l}\text { ANALYSIS } \\
\text { ROUTE }\end{array}$ \\
\hline 13 & Austenitic & $\begin{array}{l}\text { Full scale } \\
\text { gimbals feature } \\
\text { test. }\end{array}$ & $\begin{array}{l}347 \text { Casting } \\
347 \text { Weld } \\
321 \text { Tube }\end{array}$ & $\begin{array}{l}\text { Yes } \\
\text { EDM in centre } \\
\text { of weld }\end{array}$ & $\begin{array}{l}T_{\min }=20^{\circ} \mathrm{C} \\
T_{\max }=650^{\circ} \mathrm{C}\end{array}$ & $\begin{array}{l}50 \text { refuelling cycles:- } \\
\text { charge/discharge transient } \\
120 \mathrm{hr} \text { creep dwell } \\
10 \mathrm{ECD} \text { elastic cycles }\end{array}$ & $\begin{array}{l}6 \text { khrs approx. } \\
(50 \times 120 \text { hrs })\end{array}$ & $\begin{array}{l}\text { Crack size } \\
\text { acpc } \\
\text { destructive } \\
\text { examination }\end{array}$ & IJS (draft) & $\begin{array}{l}\text { R5 } \\
\text { (inelastic route) }\end{array}$ \\
\hline 14 & Transition & $\begin{array}{l}\text { EE Mk III Vessel } \\
\text { Ri }=152.5 \mathrm{~mm} \\
\mathrm{t}=27.5 \mathrm{~mm}\end{array}$ & $\begin{array}{l}\text { Transition } \\
\text { joint } \\
(2.25 \mathrm{Cr} / 316 / 3 \\
16) \\
\text { Ex-service } \\
94 \text { khrs }\end{array}$ & None & $\begin{array}{l}550^{\circ} \mathrm{C} \\
\left(540^{\circ} \mathrm{C}, 3.9\right. \\
\mathrm{MPa} \\
\text { pressure in } \\
\text { service) }\end{array}$ & $\begin{array}{l}P=11.5 \mathrm{MPa} \\
\text { Axial load }=1565 \mathrm{kN}\end{array}$ & $\begin{array}{l}11.5 \mathrm{kh} \\
\text { (initiation and growth } \\
\text { of a single semi- } \\
\text { elliptical crack in } \mathrm{HAZ} \\
\text { on the ferritic } \\
\text { interface. Failure was } \\
\text { non-catastrophic) }\end{array}$ & $\begin{array}{l}\text { Crack size } \\
\text { Ultrasonics }\end{array}$ & $\begin{array}{l}\text { O'Donnell } \\
\text { EPD/GEN/REP/0192/97 }\end{array}$ & R5 \\
\hline 15 & Transition & Inconel DMW & $\begin{array}{l}\text { Transition } \\
\text { joint } \\
(2.25 \mathrm{Cr} / \\
\text { Inconel/316) }\end{array}$ & None & $540^{\circ} \mathrm{C}$ & $\begin{array}{l}P=32 \mathrm{MPa} \\
\text { End load }=1058 \mathrm{kN}\end{array}$ & $\begin{array}{l}21,887 \mathrm{hrs} \\
\text { (Failure by initiation } \\
\text { and growth of a crack } \\
\text { at the Weld } \mathrm{E} \\
\text { interface. }\end{array}$ & $\begin{array}{l}\text { Crack size } \\
\text { Dye pen. } \\
\text { Ultrasonics }\end{array}$ & $\begin{array}{l}\text { Patel } \\
\text { EPD/GEN/REP/0282/98 }\end{array}$ & $\begin{array}{l}\text { R5, } \\
\text { Vol. } 8\end{array}$ \\
\hline 18 & Austenitic & $\begin{array}{l}\text { Welded } \\
18 \mathrm{Cr} 11 \mathrm{Ni} \text { wide } \\
\text { plate test }\end{array}$ & \begin{tabular}{|l|} 
Parent and \\
weld are 304 \\
stainless steel
\end{tabular} & None & $570^{\circ} \mathrm{C}$ & $\begin{array}{l}\text { Constant axial load }= \\
10 \mathrm{MPa} \& \text { bending cycles } \\
\text { of }+0.2 \% \text { and }-0.2 \% \text { with } \\
\text { creep dwell of } 40 \mathrm{hrs} \text {. }\end{array}$ & $\begin{array}{l}\text { Test stopped after } \\
117 \text { cycles ( } 4680 \\
\text { hrs). Evidence of } \\
\text { micro-cracking and } \\
\text { mirco-crack } \\
\text { coalescence within } \\
\text { the transverse weld } \\
\text { metal. }\end{array}$ & $?$ & $\begin{array}{l}\text { P Booth, } \\
\text { TEM/MEM/D016/95. BRITE } \\
\text { EURAM C-FAT, PROJECT } \\
\text { NO: BE 5245, Task 5. }\end{array}$ & $\begin{array}{l}\text { R5, Vols. } 2 \text { and } \\
3\end{array}$ \\
\hline
\end{tabular}




\begin{tabular}{|c|c|c|c|c|c|c|c|c|c|c|}
\hline No & TYPE & $\begin{array}{c}\text { TEST } \\
\text { DESCRIPTION }\end{array}$ & MATERIAL & $\begin{array}{l}\text { STARTER } \\
\text { CRACK(S) }\end{array}$ & TEMP & LOADING & DURATION & $\begin{array}{l}\text { MONITORING } \\
\text { INFORMATION }\end{array}$ & REFERENCES & $\begin{array}{l}\text { ANALYSIS } \\
\text { ROUTE }\end{array}$ \\
\hline 17 & Austenitic & $\begin{array}{l}\text { Welded } \\
18 \mathrm{Cr} 11 \mathrm{Ni} \text { wide } \\
\text { plate test }\end{array}$ & $\begin{array}{l}\text { German } \\
\text { designation } \\
\text { X6 Cr Ni } 18 \\
11 . \text { DIN no. } \\
1.4948 .\end{array}$ & None & $570^{\circ} \mathrm{C}$ & $\begin{array}{l}\text { Constant axial load }= \\
10 \mathrm{MPa} \& \text { bending cycles } \\
\text { of }+0.2 \% \text { and }-0.2 \% \text { with } \\
\text { creep dwell of } 40 \mathrm{hrs} \text {. }\end{array}$ & $\begin{array}{l}\text { Test stopped after } \\
117 \text { cycles ( } 4680 \\
\text { hrs). Evidence of } \\
\text { micro-cracking and } \\
\text { mirco-crack } \\
\text { coalescence within } \\
\text { the transverse weld } \\
\text { metal. }\end{array}$ & ? & $\begin{array}{l}\text { U. Lohse, S-KWU } \\
\text { NLS3/93/0079. BRITE- } \\
\text { EURAM C-FAT, PROJECT } \\
\text { NO: BE 5245, Task 5. }\end{array}$ & $\begin{array}{l}\text { Siemens/ } \\
\text { INTERATOM } \\
\text { procedure. }\end{array}$ \\
\hline 18 & Austenitic & $\begin{array}{l}\text { Welded } \\
18 \mathrm{Cr} 11 \mathrm{Ni} \text { wide } \\
\text { plate test }\end{array}$ & & & & & & & & \\
\hline 18 & Ferritic & $\begin{array}{l}\text { BRITE } 2341 \\
\text { Circumferentially } \\
\text { welded vessel } \\
\text { diXt }=126 \times 85 \\
\text { (Question - what } \\
\text { does this mean? } \\
\text { l.e is it internal } \\
\text { diameter?) }\end{array}$ & PQ1 & ? & $600^{\circ} \mathrm{C}$ & $49 \mathrm{MPa}$ & 8000 hrs & $\begin{array}{l}\text { Strain } \\
\text { measurements }\end{array}$ & $\begin{array}{l}\text { OBTAINED FROM } \\
\text { list_tommy_13_03_02.doc, } \\
\text { (WG4/meeting } 3 \text { folder in } \\
\text { my_f_disk) }\end{array}$ & ? \\
\hline 20 & Ferritic & $\begin{array}{l}\text { BE-1702 HIDA } \\
\text { bendings } \\
\text { diXt }=190 \times 23 \\
\text { (same question } \\
\text { as above) - } \\
\text { units? four } \\
\text { vessels two P91 } \\
\text { and two P22 }\end{array}$ & P91/P22 & ? & $\begin{array}{l}565^{\circ} \mathrm{Cl} \\
625^{\circ} \mathrm{C}\end{array}$ & $U p$ to $25 \mathrm{MPa}$ & Up to $8400 \mathrm{hrs}$ & $\begin{array}{l}\text { Creep crack growth } \\
\text { and strain }\end{array}$ & $\begin{array}{l}\text { OBTAINED FROM } \\
\text { list_tommy_13_03_02.doc, } \\
\text { (WG4/meeting } 3 \text { folder in } \\
\text { my_f_disk) }\end{array}$ & $?$ \\
\hline
\end{tabular}




\begin{tabular}{|c|c|c|c|c|c|c|c|c|c|c|}
\hline No & TYPE & $\begin{array}{c}\text { TEST } \\
\text { DESCRIPTION }\end{array}$ & MATERIAL & $\begin{array}{l}\text { STARTER } \\
\text { CRACK(S) }\end{array}$ & TEMP & LOADING & DURATION & $\begin{array}{l}\text { MONITORING } \\
\text { INFORMATION }\end{array}$ & REFERENCES & $\begin{array}{c}\text { ANALYSIS } \\
\text { ROUTE }\end{array}$ \\
\hline 21 & Ferritic & $\begin{array}{l}\text { Belgian vessel } \\
\text { Circumferentially } \\
\text { welded vessel } \\
\text { diXt }=240 \times 30 \\
\text { (same question } \\
\text { as above) - } \\
\text { units? } 3 \text { vessels }\end{array}$ & $\mathrm{P} 91 / \mathrm{P} 22$ & $?$ & Up to $610^{\circ} \mathrm{C}$ & Up to $20 \mathrm{MPa}$ & Up to $10000 \mathrm{hrs}$ & $\begin{array}{l}\text { Strain } \\
\text { measurements }\end{array}$ & $\begin{array}{l}\text { OBTAINED FROM } \\
\text { list_tommy_13_03_02.doc, } \\
\text { (WG4/meeting } 3 \text { folder in } \\
\text { my_f_disk) }\end{array}$ & ? \\
\hline 22 & Ferritic & $\begin{array}{l}\text { BW48 Mitsui } \\
\text { babcock T- } \\
\text { junction and } \\
\text { circomferentially } \\
\text { w. Di=210 } \\
\text { units? } \\
\text { vessels, data not } \\
\text { complete }\end{array}$ & $1 \mathrm{Cr} 1 / 2 \mathrm{Mo}$ & $?$ & $?$ & $?$ & $?$ & $?$ & $\begin{array}{l}\text { OBTAINED FROM } \\
\text { list_tommy_13_03_02.doc, } \\
\text { (WG4/meeting3 folder in } \\
\text { my_f_disk) }\end{array}$ & $?$ \\
\hline 23 & & $\begin{array}{l}\text { MPA bending } \\
\text { bendings } \\
\text { diXt }=175 \times 30 \\
\text { (what does this } \\
\text { mean and what } \\
\text { are the units?) } \\
2 \text { vessels, only } \\
\text { paper available }\end{array}$ & $\begin{array}{l}14 \mathrm{MoV} 63 \\
\text { and } \times 20\end{array}$ & ? & $550^{\circ} \mathrm{C}$ & ? & Up to $25000 \mathrm{hrs}$ & $\begin{array}{l}\text { Strain } \\
\text { measurements }\end{array}$ & $\begin{array}{l}\text { OBTAINED FROM } \\
\text { list_tommy_13_03_02.doc, } \\
\text { (WG4/meeting } 3 \text { folder in } \\
\text { my_f_disk) }\end{array}$ & ? \\
\hline
\end{tabular}




\begin{tabular}{|c|c|c|c|c|c|c|c|c|c|c|}
\hline No & TYPE & $\begin{array}{c}\text { TEST } \\
\text { DESCRIPTION }\end{array}$ & MATERIAL & $\begin{array}{l}\text { STARTER } \\
\text { CRACK(S) }\end{array}$ & TEMP & LOADING & DURATION & $\begin{array}{l}\text { MONITORING } \\
\text { INFORMATION }\end{array}$ & REFERENCES & $\begin{array}{l}\text { ANALYSIS } \\
\text { ROUTE }\end{array}$ \\
\hline 24 & & $\begin{array}{l}\text { Pipe bend tests } \\
\mathrm{Ri}=225 \mathrm{~mm} \\
\mathrm{t}=20 \mathrm{~mm} \\
90^{\circ} \text { bend }\end{array}$ & $\begin{array}{l}\mathrm{P} 91 \\
\text { (P91/B1) } \\
\text { static end- } \\
\text { constraint }\end{array}$ & $\begin{array}{l}\text { In longitudinal } \\
\text { direction: } \\
3 \text { at bend } \\
\text { extrados } X, Y \text {. } \\
Z \\
3 \text { in neutral } \\
\text { axis Q. R }\end{array}$ & $625^{\circ} \mathrm{C}$ & $\begin{array}{l}15 \mathrm{MPa} \\
20 \mathrm{MPa}\end{array}$ & $\begin{array}{l}1300 \text { hrs (no cracks) } \\
1460 \text { hrs (stopped } \\
\text { without failure) }\end{array}$ & $\begin{array}{l}\text { Thermocouples } \\
\text { Load deflection } \\
\text { measurements } \\
\text { AC-PD (CCG) } \\
\text { HT strain gauges } \\
\text { post test exam.: } \\
\text { Metallography a.o. }\end{array}$ & BE-1702 HIDA & $\begin{array}{l}\text { Two-Criteria } \\
\text { (CC initiation) } \\
\mathrm{C}^{\times} \text {, sigamref, K } \\
\text { acc. BS7910 } \\
\text { (CCG) }\end{array}$ \\
\hline 25 & & $\begin{array}{l}\text { Pipe bend tests } \\
\mathrm{Ri}=225 \mathrm{~mm} \\
\mathrm{t}=20 \mathrm{~mm} \\
90^{\circ} \text { bend }\end{array}$ & $\begin{array}{l}\text { P91 } \\
\text { (P91/B2) } \\
\text { static without } \\
\text { constraint }\end{array}$ & $\begin{array}{l}\text { In longitudinal } \\
\text { direction: } \\
3 \text { at bend } \\
\text { extrados } X, Y \text {, } \\
Z \\
3 \text { in neutral } \\
\text { axis } Q . R\end{array}$ & $625^{\circ} \mathrm{C}$ & $16 \mathrm{MPa}$ & 2840 hrs & $\begin{array}{l}\text { Thermocouples } \\
\text { Load deflection } \\
\text { measurements } \\
\text { AC-PD (CCG) } \\
\text { HT strain gauges } \\
\text { post test exam.: } \\
\text { Metallography a.o. }\end{array}$ & BE-1702 HIDA & $\begin{array}{l}\text { Two-Criteria } \\
\text { (CC initiation) } \\
\mathrm{C}^{\mathrm{x}} \text {, sigmaref, K } \\
\text { acc. BS7910 } \\
\text { (CCG) }\end{array}$ \\
\hline 28 & & $\begin{array}{l}\text { Pipe bend tests } \\
\mathrm{Ri}=156 \mathrm{~mm} \\
\mathrm{t}=20 \mathrm{~mm} \\
90^{\circ} \text { bend }\end{array}$ & $\begin{array}{l}\text { P22 } \\
\text { (P22/B1) } \\
\text { static end- } \\
\text { constraint }\end{array}$ & $\begin{array}{l}\text { In longitudinal } \\
\text { direction: } \\
3 \text { at bend } \\
\text { extrados } X, Y \text {, } \\
Z \\
3 \text { in neutral } \\
\text { axis Q. R }\end{array}$ & $565^{\circ} \mathrm{C}$ & $\begin{array}{l}15 \mathrm{MPa} \\
20 \mathrm{MPa}\end{array}$ & $\begin{array}{l}1300 \text { hrs (no cracks) } \\
8440 \text { hrs }\end{array}$ & $\begin{array}{l}\text { Thermocouples } \\
\text { Load deflection } \\
\text { measurements } \\
\text { AC-PD (CCG) } \\
\text { HT strain gauges } \\
\text { post test exam.: } \\
\text { Metallography a.o. }\end{array}$ & BE-1702 HIDA & $\begin{array}{l}\text { Two-Criteria } \\
\text { (CC initiation) } \\
C^{\times} \text {sigmaref, } \mathrm{K} \\
\text { acc. BS7910 } \\
\text { (CCG) }\end{array}$ \\
\hline
\end{tabular}




\begin{tabular}{|c|c|c|c|c|c|c|c|c|c|c|}
\hline No & TYPE & $\begin{array}{c}\text { TEST } \\
\text { DESCRIPTION }\end{array}$ & MATERIAL & $\begin{array}{l}\text { STARTER } \\
\text { CRACK(S) }\end{array}$ & TEMP & LOADING & DURATION & $\begin{array}{l}\text { MONITORING } \\
\text { INFORMATION }\end{array}$ & REFERENCES & $\begin{array}{l}\text { ANALYSIS } \\
\text { ROUTE }\end{array}$ \\
\hline 27 & & $\begin{array}{l}\text { Pipe bend tests } \\
\mathrm{Ri}=156 \mathrm{~mm} \\
\mathrm{t}=20 \mathrm{~mm} \\
90^{\circ} \text { bend }\end{array}$ & $\begin{array}{l}\text { P22 } \\
\text { (P22/B2) } \\
\text { static without } \\
\text { constraint }\end{array}$ & $\begin{array}{l}\text { In longitudinal } \\
\text { direction: } \\
3 \text { at bend } \\
\text { extrados } X, Y \text {, } \\
Z \\
3 \text { in neutral } \\
\text { axis } Q . R\end{array}$ & $565^{\circ} \mathrm{C}$ & $25 \mathrm{MPa}$ & 3600 hrs & $\begin{array}{l}\text { Thermocouples } \\
\text { Load deflection } \\
\text { measurements } \\
\text { AC-PD (CCG) } \\
\text { HT strain gauges } \\
\text { post test exam.: } \\
\text { Metallography a.o. }\end{array}$ & BE- 1702 HIDA & $\begin{array}{l}\text { Two-Criteria } \\
\text { (CC initiation) } \\
\mathrm{C}^{\mathrm{x}} \text {. sigmaref, } \mathrm{K} \\
\text { acc. BS7910 } \\
\text { (CCG) }\end{array}$ \\
\hline 28 & & $\begin{array}{l}\text { 4-Point bending } \\
\mathrm{Ri}=156 \mathrm{~mm} \\
\mathrm{t}=20 \mathrm{~mm}\end{array}$ & $\begin{array}{l}\text { P91 } \\
\text { (P91/P1) } \\
\text { Creep Crack } \\
\text { Growth CCG }\end{array}$ & $\begin{array}{l}\text { In circumfer. } \\
\text { direction: } \\
2 \text { at parent } \\
\text { metal (A, C) } \\
1 \text { in } \mathrm{HAZ}(\mathrm{B})\end{array}$ & $625^{\circ} \mathrm{C}$ & $\begin{array}{l}\mathrm{Pi}=20 \mathrm{MPa} \\
\text { Fext. }=96 \mathrm{kN} \\
\mathrm{Pi}=20 \mathrm{MPa} \\
\text { Fext. }=120 \mathrm{kN}\end{array}$ & $\begin{array}{l}3648 \text { hrs (no crack } \\
\text { growth) } \\
1440 \text { hrs (leakage in } \\
\text { weld) }\end{array}$ & $\begin{array}{l}\text { Thermocouples } \\
\text { Load deflection } \\
\text { measurements } \\
\text { AC-PD (CCG) } \\
\text { HT strain gauges } \\
\text { (notch opening) } \\
\text { post test exam.: } \\
\text { Metallography a.o. }\end{array}$ & BE-1702 HIDA & $\begin{array}{l}\text { Two-Criteria } \\
\text { (CC initiation) } \\
\mathrm{C}^{\times} \text {. sigmaref, } \mathrm{K} \\
\text { acc. BS7910 } \\
\text { (CCG) }\end{array}$ \\
\hline 28 & & $\begin{array}{l}\text { 4-Point bending } \\
\mathrm{Ri}=156 \mathrm{~mm} \\
\mathrm{t}=20 \mathrm{~mm}\end{array}$ & $\begin{array}{l}\text { P91 } \\
\text { (P91/P2) } \\
\text { creep/fatigue } \\
\text { crack growth } \\
\text { CFG }\end{array}$ & $\begin{array}{l}\text { In circumfer. } \\
\text { direction: } \\
2 \text { at parent } \\
\text { metal (A, C) } \\
1 \text { in HAZ (B) }\end{array}$ & $625^{\circ} \mathrm{C}$ & $\begin{array}{l}\mathrm{Pi}=20 \mathrm{MPa} \\
\text { deltaFext. }=96 \mathrm{kN} \\
\mathrm{Pi}=20 \mathrm{MPa} \\
\text { deltaFext. }=120 \mathrm{kN} \\
\mathrm{f}=0.001 \mathrm{~Hz}\end{array}$ & $\begin{array}{l}3648 \mathrm{~h} / 12740 \mathrm{cycl} \text {. } \\
\text { (no crack growth) } \\
1440 \mathrm{~h} / 4634 \mathrm{cycl} \text {. }\end{array}$ & $\begin{array}{l}\text { Thermocouples } \\
\text { Load deflection } \\
\text { measurements } \\
\text { AC-PD (CCG) } \\
\text { HT strain gauges } \\
\text { post test exam.: } \\
\text { Metallography a.o. }\end{array}$ & BE- 1702 HIDA & $\begin{array}{l}\text { Two-Criteria } \\
\text { (CC initiation) } \\
\mathrm{C}^{\mathrm{x}} \text {, sigmaref, K } \\
\text { acc. BS7910 } \\
\text { (CCG) }\end{array}$ \\
\hline
\end{tabular}




\begin{tabular}{|c|c|c|c|c|c|c|c|c|c|c|}
\hline No & TYPE & $\begin{array}{c}\text { TEST } \\
\text { DESCRIPTION }\end{array}$ & MATERIAL & $\begin{array}{l}\text { STARTER } \\
\text { CRACK(S) }\end{array}$ & TEMP & LOADING & DURATION & $\begin{array}{l}\text { MONITORING } \\
\text { INFORMATION }\end{array}$ & REFERENCES & $\begin{array}{l}\text { ANALYSIS } \\
\text { ROUTE }\end{array}$ \\
\hline 30 & & $\begin{array}{l}\text { 4-Point bending } \\
\mathrm{Ri}=156 \mathrm{~mm} \\
\mathrm{t}=20 \mathrm{~mm}\end{array}$ & $\begin{array}{l}\text { P22 } \\
(\mathrm{P} 22 / \mathrm{P} 1) \\
\text { Creep Crack } \\
\text { Growth CCG }\end{array}$ & $\begin{array}{l}\text { In circumfer. } \\
\text { direction: } \\
2 \text { at parent } \\
\text { metal (A, C) } \\
1 \text { in } \mathrm{HAZ}(\mathrm{B})\end{array}$ & $565^{\circ} \mathrm{C}$ & $\begin{array}{l}\mathrm{Pi}=20 \mathrm{MPa} \\
\text { Fext. }=47 \mathrm{kN} \\
\mathrm{Pi}=20 \mathrm{MPa} \\
\text { Fext. }=90 \mathrm{kN}\end{array}$ & $\begin{array}{l}5020 \text { h(no crack } \\
\text { growth) } \\
2800 \text { h(test stop) }\end{array}$ & $\begin{array}{l}\text { Thermocouples } \\
\text { Load deflection } \\
\text { measurements } \\
\text { AC-PD (CCG) } \\
\text { post test exam.: } \\
\text { Metallography a.o. }\end{array}$ & BE-1702 HIDA & $\begin{array}{l}\text { Two-Criteria } \\
\text { (CC initiation) } \\
\mathrm{C}^{\times} \text {, sigmaref, K } \\
\text { acc. BS7910 } \\
\text { (CCG) }\end{array}$ \\
\hline 31 & & $\begin{array}{l}\text { 4-Point bending } \\
\mathrm{Ri}=156 \mathrm{~mm} \\
\mathrm{t}=20 \mathrm{~mm}\end{array}$ & $\begin{array}{l}\text { P22 } \\
\text { (P22/P2) } \\
\text { Creep/Fatigue } \\
\text { Crack Growth } \\
\text { CFG }\end{array}$ & $\begin{array}{l}\text { In circumfer. } \\
\text { direction: } \\
2 \text { at parent } \\
\text { metal (A, C) } \\
1 \text { in HAZ (B) }\end{array}$ & $565^{\circ} \mathrm{C}$ & $\begin{array}{l}\mathrm{Pi}=20 \mathrm{MPa} \\
\text { deltaFext. }=47 \mathrm{kN} \\
\mathrm{Pi}=20 \mathrm{MPa} \\
\text { deltaFext }=90 \mathrm{kN} \\
\mathrm{f}=0.001 \mathrm{~Hz}\end{array}$ & $\begin{array}{l}5020 \mathrm{~h} / 16420 \mathrm{cycl} \\
\text { (no crack growth) } \\
2800 \mathrm{~h} / 9655 \mathrm{cycl} . \\
\text { (test stop) }\end{array}$ & $\begin{array}{l}\text { Thermocouples } \\
\text { Load deflection } \\
\text { measurements } \\
\text { AC-PD (CCG) } \\
\text { post test exam.: } \\
\text { Metallography a.o. }\end{array}$ & BE-1702 HIDA & $\begin{array}{l}\text { Two-Criteria } \\
\text { (CC initiation) } \\
\mathrm{C}^{x} \text {, sigmaref, } \mathrm{K} \\
\text { acc. BS7910 } \\
\text { (CCG) }\end{array}$ \\
\hline 32 & Ferritic & $\begin{array}{l}\text { SENB specimen } \\
\text { Length }=450 \mathrm{~mm} \\
\text { Width }=100 \mathrm{~mm} \\
\mathrm{t}=75 \mathrm{~mm}\end{array}$ & Cast $1 \mathrm{CrMoV}$ & $\begin{array}{l}\text { 6mm radius, } \\
\text { single edged, } \\
\text { semi-circular } \\
\text { section notch } \\
\text { on the } 100 \mathrm{~mm} \\
\text { face }\end{array}$ & $550^{\circ} \mathrm{C}$ & 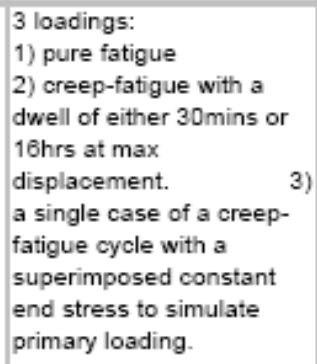 & $\begin{array}{l}10 \text { tests performed - } \\
\text { ranged from } 153 \text { to } \\
4000 \text { cylcles }\end{array}$ & $\begin{array}{l}\text { Direct current } \\
\text { potential drop } \\
\text { technique (DCPD) }\end{array}$ & $\begin{array}{l}\text { BRITE-EURAM C-FAT, } \\
\text { Task } 5 \text { Milestone report. } \\
\text { Development \& validation of } \\
\text { creep-fatigue assessment } \\
\text { methodologies (AEAT - } \\
0483 \text { ) }\end{array}$ & Volume 3 of R5 \\
\hline
\end{tabular}




\begin{tabular}{|c|c|c|c|c|c|c|c|c|c|c|}
\hline No & TYPE & $\begin{array}{c}\text { TEST } \\
\text { DESCRIPTION }\end{array}$ & MATERIAL & $\begin{array}{l}\text { STARTER } \\
\text { CRACK(S) }\end{array}$ & TEMP & LOADING & DURATION & $\begin{array}{l}\text { MONITORING } \\
\text { INFORMATION }\end{array}$ & REFERENCES & $\begin{array}{l}\text { ANALYSIS } \\
\text { ROUTE }\end{array}$ \\
\hline 33 & Ferritic & $\begin{array}{l}\text { SENB specimen } \\
\text { Length }=450 \mathrm{~mm} \\
\text { Width }=100 \mathrm{~mm} \\
t=75 \mathrm{~mm}\end{array}$ & Cast $1 \mathrm{CrMoV}$ & $\begin{array}{l}\text { 6mm radius, } \\
\text { single edged, } \\
\text { semi-circular } \\
\text { section notch } \\
\text { on the } 100 \mathrm{~mm} \\
\text { face }\end{array}$ & $550^{\circ} \mathrm{C}$ & $\begin{array}{l}3 \text { loadings: } \\
\text { 1) pure fatigue } \\
\text { 2) creep-fatigue with a } \\
\text { dwell of either } 30 \mathrm{mins} \text { or } \\
16 \mathrm{hrs} \text { at max } \\
\text { displacement. } \\
\text { 3) a single case of a creep- } \\
\text { fatigue cycle with a } \\
\text { superimposed constant } \\
\text { end stress to simulate } \\
\text { primary loading. }\end{array}$ & $\begin{array}{l}6 \text { tests analysed: } \\
\text { ranged from } 177 \text { to } \\
4000 \text { cycles }\end{array}$ & $\begin{array}{l}\text { Direct current } \\
\text { potential drop } \\
\text { technique (DCPD) }\end{array}$ & $\begin{array}{l}\text { BRITE-EURAM C-FAT, } \\
\text { Task } 5 \text { Milestone report. } \\
\text { Development \& validation of } \\
\text { creep-fatigue assessment } \\
\text { methodologies (Nuclear } \\
\text { Electric } \\
\text { EPD/GEN/REP/0095/96) }\end{array}$ & $\begin{array}{l}\text { R5 Volumes } 2 \\
\& 3\end{array}$ \\
\hline 34 & Ferritic & $\begin{array}{l}\text { LICON } 1: 1 \\
\text { Tee junction, } \\
\text { Ri }=142.5 \mathrm{~mm} \text {, } \\
\mathrm{t}=21.925 \mathrm{~mm}\end{array}$ & $\begin{array}{l}\text { E011. } \\
\text { Thyssen } \\
\text { MTS011 weld } \\
\text { metal }\end{array}$ & None & $625^{\circ} \mathrm{C}$ & $\begin{array}{l}\mathrm{P} 1=10.21 \mathrm{MPa} \\
\mathrm{P} 2=5.88 \mathrm{MPa} \\
\mathrm{P} 3=8.5 \mathrm{MPa}\end{array}$ & $\begin{array}{l}\text { P1: } 1078 \mathrm{hr} \text { ( } 90^{\circ} \text { flank } \\
7.5 \mathrm{~mm} \text { deep defect) } \\
\text { P2: } 620 \mathrm{hr} \\
\text { P3: } 4482 \mathrm{hr}\left(90^{\circ} \text { flank }\right. \\
\text { indication), } 7218 \mathrm{hr} \\
\left(90^{\circ} \text { flank } 150 \mathrm{~mm},\right. \\
0.5 \mathrm{~mm} \text { defect) }\end{array}$ & $\begin{array}{l}\text { Creep Strain: Strain } \\
\text { gauges, creep pips } \\
\text { Crack size: ACPD, } \\
\text { ultrasonics, post } \\
\text { test examination }\end{array}$ & $\begin{array}{l}\text { Russell, G, Hack, R, } \\
\text { Fleming, A and McMillan, G } \\
\text { LICON: Task } 5 \text { Final Report } \\
\text { Mitsui Babcock Report } \\
\text { E/O0/115 }\end{array}$ & ? \\
\hline \multirow[t]{2}{*}{35} & \multirow[b]{2}{*}{ Ferritic } & \multirow[b]{2}{*}{$\begin{array}{l}\text { HIDA seam- } \\
\text { welded straight } \\
\text { pipe }\end{array}$} & \multirow[b]{2}{*}{$\begin{array}{l}2.25 \mathrm{Cr} 1 \mathrm{Mo} \\
\text { Parent \& } \\
\text { welds }\end{array}$} & \multirow{2}{*}{$\begin{array}{l}3 \text { EDM semi- } \\
\text { elliptical } \\
\text { defects } \\
\text { machined one } \\
\text { in the HAZ and } \\
2 \text { in the base } \\
\text { material }\end{array}$} & \multirow[b]{2}{*}{$585^{\circ} \mathrm{C}$} & $\begin{array}{l}\text { Static pressure } \\
p=15 \mathrm{MPa} \\
p=17.5 \mathrm{MPa}\end{array}$ & $\begin{array}{l}5.88 \mathrm{kH} \\
4.32 \mathrm{kH}\end{array}$ & \multirow{2}{*}{$\begin{array}{l}\text { Thremocouples, } \\
\text { DC-PD gauges } \\
\text { Capacitance } \\
\text { gauges }\end{array}$} & \multirow{2}{*}{$\begin{array}{l}\text { Le Mat Hamata \& Shibli (a) } \\
\text { Int. J. Pres. Vess. \& Piping. } \\
\text { 78, } 819-826 \text { 2001. (b) Int. } \\
\text { J. Pres. Vess. \& Piping. } 778 . \\
\text { 827-836 2001. }\end{array}$} & \multirow[b]{2}{*}{ R5, BS7910 } \\
\hline & & & & & & $\begin{array}{l}\text { Cyclic pressure } \\
p=0-17.5 \mathrm{MPa} \\
\mathrm{p}=0-20 \mathrm{MPa} \\
\text { freq }=10^{-4}\end{array}$ & $\begin{array}{l}4.62 \mathrm{kH}-1540 \mathrm{cycl} \\
1.60 \mathrm{kH}-533 \mathrm{cycl}\end{array}$ & & & \\
\hline \multirow[t]{2}{*}{36} & \multirow[b]{2}{*}{ Bainitic } & \multirow{2}{*}{$\begin{array}{l}\text { HIDA seam- } \\
\text { welded straight } \\
\text { pipe }\end{array}$} & \multirow[b]{2}{*}{$\begin{array}{c}\text { P91 Parent \& } \\
\text { welds }\end{array}$} & \multirow{2}{*}{$\begin{array}{c}3 \text { spark } \\
\text { machined } \\
\text { defects, one in } \\
\text { the } \mathrm{HAZ} \text { and } \\
\text { two in the base } \\
\text { material }\end{array}$} & \multirow[b]{2}{*}{$625^{\circ} \mathrm{C}$} & $\begin{array}{l}\text { Static pressure } \\
p=15 \mathrm{MPa}\end{array}$ & $1.43 \mathrm{kH}$ & \multirow{2}{*}{$\begin{array}{l}\text { Thremocouples, } \\
\text { DC-PD gauges } \\
\text { Capacitance } \\
\text { gauges }\end{array}$} & \multirow{2}{*}{$\begin{array}{l}\text { Le Mat Hamata \& Shibli (a) } \\
\text { Int. J. Pres. Vess. \& Piping. } \\
\text { 78, } 819-826 \text { 2001. (b) Int. } \\
\text { J. Pres. Vess. \& Piping. 778, } \\
\text { 827-836 2001. }\end{array}$} & \multirow[b]{2}{*}{ R5, BS7911 } \\
\hline & & & & & & $\begin{array}{l}\text { Cyclic pressure } \\
p=0-11 \mathrm{MPa} \\
\text { freq }=10^{-4}\end{array}$ & $5.55 \mathrm{kH}-1850 \mathrm{cycl}$ & & & \\
\hline
\end{tabular}

